\title{
Proton-responsive Ruthenium(II) Catalysts for the Solvolysis of Ammonia-Borane.
}

Virginia San Nacianceno, ${ }^{\dagger}$ María A. Garralda,${ }^{\dagger}$ Jon M. Matxain, ${ }^{\dagger *}$ and Zoraida Freixa* ${ }^{\dagger \S}$

$\dagger$ Faculty of Chemistry, University of the Basque Country (UPV-EHU), San Sebastián, 20018, Spain

$¥$ Donostia International Physics Center (DIPC), San Sebastián, 20018, Spain

$\S$ IKERBASQUE, Basque Foundation for Science, Bilbao, 48011, Spain 


\section{CONTENTS:}

\section{Study of the $\mathrm{pH}$ response of compounds 1,2 and $\mathrm{V}$.}

Figure S1. a) UV-vis absorption spectra of $\left[\mathrm{Ru}(\mathrm{p}-\mathrm{Cym})(\mathrm{dhbp})\left(\mathrm{H}_{2} \mathrm{O}\right)\right](\mathrm{OTf})_{2}(2)$ in a $\mathrm{pH}$ titration in $\mathrm{H}_{2} \mathrm{O}$. b) Absorbance changes at single wavelengths as a function of $\mathrm{pH}$. $-56$

Figure S2. a) UV-vis absorption spectra of $[\mathrm{Ru}(\mathrm{p}-\mathrm{Cym})(\mathrm{dhbp}) \mathrm{Cl}] \mathrm{Cl}(\mathbf{1})$ in a pH titration in $\mathrm{H}_{2} \mathrm{O}$. b) Absorbance changes at single wavelengths as a function of $\mathrm{pH}$

Figure S3. a) UV-vis absorption spectra of $[\mathrm{Ru}(\mathrm{p}-\mathrm{Cym})($ bipy $) \mathrm{C}] \mathrm{Cl}(\mathbf{V})$ in a $\mathrm{pH}$ titration in $\mathrm{H}_{2} \mathrm{O}$. b) Absorbance changes at single wavelengths as a function of $\mathrm{pH}$. Botzmann fits used to calculate the corresponding $\mathrm{pH}$ inflexion points are plotted using discontinuous lines.

Figure S4. ${ }^{1} \mathrm{H}$ NMR spectra $(300 \mathrm{MHz})$ of $\left[\mathrm{Ru}(\mathrm{p}-\mathrm{Cym})(\mathrm{dhbp})\left(\mathrm{H}_{2} \mathrm{O}\right)\right](\mathrm{OTf})_{2}(2)$ in a pH titration in $\mathrm{H}_{2} \mathrm{O}$ (internal capillary of $\mathrm{D}_{2} \mathrm{O}$ ). -S9

Figure S5. ${ }^{1} \mathrm{H}$ NMR spectra $(300 \mathrm{MHz})$ of $[\mathrm{Ru}(\mathrm{p}-\mathrm{Cym})(\mathrm{dhbp}) \mathrm{Cl}] \mathrm{Cl}(\mathbf{1})$ in a pH titration in $\mathrm{H}_{2} \mathrm{O}$ (internal capillary of $\mathrm{D}_{2} \mathrm{O}$ ). S10

\section{Catalytic experiments: reaction profiles.}

Figure S6. Reaction profile of $A B$ hydrolysis using $[R u(p-C y m)(d h b p) C l] C l(1)$ as precatalyst. (Table 1, Entry 1). Conditions: $[\mathrm{Ru}]=2.3 \mathrm{mM},[A B]=0.46 \mathrm{M}, 1.5 \mathrm{~mL} \mathrm{H} \mathrm{O}_{2}, 30^{\circ} \mathrm{C}$. S11

Figure S7. Reaction profile of $A B$ hydrolysis using $\left[R u(p-C y m)(d h b p)\left(\mathrm{H}_{2} \mathrm{O}\right)\right](\mathrm{OTf})_{2}$ (2) as precatalyst. (Table 1, Entry 2). Conditions: $[\mathrm{Ru}]=2.3 \mathrm{mM},[\mathrm{AB}]=0.46 \mathrm{M}, 1.5 \mathrm{~mL} \mathrm{H} \mathrm{H}_{2} \mathrm{O}, 30^{\circ} \mathrm{C}$. $\mathbf{S 1 1}$

Figure S8. Reaction profile of $A B$ hydrolysis using $[\mathrm{Ru}(\mathrm{p}-\mathrm{Cym})(\mathrm{bipy}) \mathrm{Cl}] \mathrm{Cl}(\mathbf{V})$ as precatalyst. (Table 1, Entry 3). Conditions: $[\mathrm{Ru}]=2.3 \mathrm{mM},[\mathrm{AB}]=0.46 \mathrm{M}, 1.5 \mathrm{~mL} \mathrm{H} \mathrm{O}, 30^{\circ} \mathrm{C}$. S12

Figure S9. Reaction profile of $A B$ hydrolysis using $[\operatorname{IrCp}(\mathrm{dhbp}) \mathrm{Cl}] \mathrm{Cl}$ (Himeda's catalyst) (VI) as precatalyst. (Table 1, Entry 4). Conditions: $[\mathrm{Ir}]=2.3 \mathrm{mM},[\mathrm{AB}]=0.46 \mathrm{M}, 1.5 \mathrm{~mL} \mathrm{H} \mathrm{O}_{2} \mathrm{O}, 3{ }^{\circ} \mathrm{C}$.- $\mathbf{S 1 2}$

Figure S10. Reaction profiles of successive $A B$ hydrolysis using $[\mathrm{Ru}(\mathrm{p}-\mathrm{Cym})(\mathrm{dhbp}) \mathrm{Cl}] \mathrm{Cl}(\mathbf{1})$ as precatalyst. (Table 1, Entry 5). First-order kinetic adjustment for the second addition shown. Conditions: $[\mathrm{Ru}]=2.3 \mathrm{mM},[\mathrm{AB}]=0.46 \mathrm{M}, 1.5 \mathrm{~mL} \mathrm{H} \mathrm{O}_{2}, 60^{\circ} \mathrm{C}$.

Figure S11. Reaction profile of $A B$ hydrolysis using $\left[\mathrm{Ru}(\mathrm{p}-\mathrm{Cym})(\mathrm{dhbp})\left(\mathrm{H}_{2} \mathrm{O}\right)\right](\mathrm{OTf})_{2}$ (2) as precatalyst. (Table 1 entry 6). Conditions: $[\mathrm{Ru}]=2.3 \mathrm{mM},[\mathrm{AB}]=0.46 \mathrm{M}, 1.5 \mathrm{~mL} \mathrm{H} \mathrm{O}_{2}, 60^{\circ} \mathrm{C}$.-S14

Figure S12. Reaction profiles of successive $A B$ hydrolysis using $[\mathrm{Ru}(\mathrm{p}-\mathrm{Cym})(\mathrm{bipy}) \mathrm{Cl}] \mathrm{Cl}(\mathbf{V})$ as precatalyst. (Table 1 entry 7). Conditions: $[R u]=2.3 \mathrm{mM},[A B]=0.46 \mathrm{M}, 1.5 \mathrm{~mL} \mathrm{H} \mathrm{O}_{2}, 60^{\circ} \mathrm{C} .-\mathrm{S} 15$

Figure S13. Reaction profiles of successive $A B$ hydrolysis using $\left[\operatorname{IrCp} *\left(\right.\right.$ bipy-OH) $\left.\left(\mathrm{H}_{2} \mathrm{O}\right)\right] \mathrm{SO}_{4}$ (Himeda's catalyst) (VI). (Table 1, Entry 8). Conditions: $[\mathrm{Ir}]=2.3 \mathrm{mM},[\mathrm{AB}]=0.46 \mathrm{M}, 1.5 \mathrm{~mL}$ $\mathrm{H} 2 \mathrm{O}, 60^{\circ} \mathrm{C}$. $-\mathrm{S} 16$

Figure S14. Reaction profile of $A B$ hydrolysis using $[\mathrm{Ru}(\mathrm{p}-\mathrm{Cym})(\mathrm{dmobp}) \mathrm{Cl}] \mathrm{Cl}\left(\mathrm{dmobp}=6,6^{\prime}\right.$ dimethoxybipyridine) as precatalyst. Sigmoidal fitting shown. (not discussed in manusctipt). Conditions: $[\mathrm{Ru}]=2.3 \mathrm{mM},[\mathrm{AB}]=0.46 \mathrm{M}, 1.5 \mathrm{~mL} \mathrm{H} 2 \mathrm{O}, 30^{\circ} \mathrm{C}$.

Figure S15. Reaction profiles of successive $A B$ hydrolysis using $[\mathrm{Ru}(\mathrm{p}-\mathrm{Cym})(\mathrm{dmobp}) \mathrm{Cl}] \mathrm{Cl}$ as precatalyst. (not shown in manuscript). Conditions: $[R u]=2.3 \mathrm{mM},[A B]=0.46 \mathrm{M}, 1.5 \mathrm{~mL} \mathrm{H} \mathrm{H}_{2} \mathrm{O}$, $60{ }^{\circ} \mathrm{C}$. 
Figure S16. Reaction profiles of $A B$ hydrolysis using $[\mathrm{Ru}(\mathrm{p}-\mathrm{Cym})(\mathrm{dmobp}) \mathrm{Cl}] \mathrm{Cl}$ as precatalyst. (not shown in manuscript). Conditions: $[\mathrm{Ru}]=2.3 \mathrm{mM},[\mathrm{AB}]=0.46 \mathrm{M}, 1.5 \mathrm{~mL} \mathrm{H} \mathrm{O}_{2}, 60^{\circ} \mathrm{C}$. A reaction profile of a run in which $\mathrm{Hg}$ (1000 eq. per Ru) was added after liberation of 1 eq. of $\mathrm{H}_{2}$ has been superimposed.

$\mathrm{S} 19$

Figure S17. Reaction profiles of $A B$ hydrolysis using $[\mathrm{Ru}(\mathrm{p}-\mathrm{Cym})(\mathrm{dhbp}) \mathrm{Cl}] \mathrm{Cl}(\mathbf{1})$ as precatalyst. (Table 2, Entry 1). Conditions: $[\mathrm{Ru}]=2.3 \mathrm{mM},[\mathrm{AB}]=0.46 \mathrm{M}, 1.5 \mathrm{~mL}$ of a $\mathrm{NH}_{3}$ solution $0.46 \mathrm{M}$ in $\mathrm{H}_{2} \mathrm{O}, 60^{\circ} \mathrm{C}$.

Figure S18. Reaction profiles of successive $A B$ solvolysis using $[R u(p-C y m)(d h b p) C l] C l(1)$. (Table 2, Entry 2). Conditions: $[\mathrm{Ru}]=2.3 \mathrm{mM},[\mathrm{AB}]=0.46 \mathrm{M}, 1.5 \mathrm{~mL} \mathrm{NaOH} / \mathrm{H}_{2} \mathrm{O}(\mathrm{pH}=9.0), 60{ }^{\circ} \mathrm{C} .-\mathrm{S} 21$

Figure S19. Reaction profiles of successive $A B$ solvolysis using $[\mathrm{Ru}(\mathrm{p}-\mathrm{Cym})(\mathrm{dhbp}) \mathrm{Cl}] \mathrm{Cl}(\mathbf{1})$. (Table 2, Entry 3). Conditions: $[\mathrm{Ru}]=2.3 \mathrm{mM},[\mathrm{AB}]=0.46 \mathrm{M}, 1.5 \mathrm{~mL} \mathrm{NaOH} / \mathrm{H}_{2} \mathrm{O} 0.46 \mathrm{M}(\mathrm{pH}=13.7), 60$ ${ }^{\circ} \mathrm{C}$.

Figure S20. Reaction profiles of successive $A B$ solvolysis using $[\mathrm{Ru}(\mathrm{p}-\mathrm{Cym})(\mathrm{dhbp}) \mathrm{Cl}] \mathrm{Cl}(\mathbf{1})$. (Table 2, Entry 4). Conditions: $[R u]=2.3 \mathrm{mM},[A B]=0.46 \mathrm{M}, 1.5 \mathrm{~mL}$ (HEPES buffer $1 \mathrm{M}$ in $\mathrm{H}_{2} \mathrm{O}, \mathrm{pH} 7.0$ ), $60^{\circ} \mathrm{C}$.

Figure S21. Reaction profiles of successive $A B$ solvolysis using $[R u(p-C y m)(d h b p) C l] C l(1)$. (Table 2, Entry 5). Conditions: $[\mathrm{Ru}]=2.3 \mathrm{mM},[\mathrm{AB}]=0.46 \mathrm{M}, 1.5 \mathrm{~mL}$ (HEPES buffer $1 \mathrm{M}$ in $\mathrm{H}_{2} \mathrm{O}, \mathrm{pH} 7.0$ ), $30^{\circ} \mathrm{C}$.

Figure S22. Reaction profiles of successive $A B$ solvolysis using $[R u(p-C y m)(d h b p) C l] C l(1)$. (Table 2, Entry 6). Conditions: $[\mathrm{Ru}]=0.1 \mathrm{mM},[\mathrm{AB}]=0.1 \mathrm{M}, 5 \mathrm{~mL}$ (HEPES buffer $1 \mathrm{M}$ in $\mathrm{H}_{2} \mathrm{O}, \mathrm{pH} 7.0$ ), $65^{\circ} \mathrm{C}$.

Figure S23. Reaction profiles of successive $A B$ solvolysis using $[R u(p-C y m)(d h b p) C l] C l(1)$. (Table 2, Entry 7). Conditions: $[\mathrm{Ru}]=0.1 \mathrm{mM},[\mathrm{AB}]=0.1 \mathrm{M}, 5 \mathrm{~mL}\left(\mathrm{H}_{2} \mathrm{O}, \mathrm{pH} 9.0\right), 65^{\circ} \mathrm{C}$. -S26

Figure S24. Reaction profile of $A B$ solvolysis using $[\mathrm{Ru}(\mathrm{p}-\mathrm{Cym})(\mathrm{dhbp}) \mathrm{Cl}] \mathrm{Cl}(\mathbf{1})$. Conditions: $[\mathrm{Ru}]$ $=2.3 \mathrm{mM},[\mathrm{AB}]=0.46 \mathrm{M}, 1.5 \mathrm{~mL}$ (phosphate buffer $1 \mathrm{M}$ in $\mathrm{H}_{2} \mathrm{O}, \mathrm{pH} 7.0$ ), $60^{\circ} \mathrm{C}$. S27

Figure S25. Reaction profiles of: TOP: AB alcoholysis using $[\mathrm{Ru}(\mathrm{p}-\mathrm{Cym})(\mathrm{dhbp}) \mathrm{Cl}] \mathrm{Cl}(\mathbf{1})$. (Table 2, Entry 8). Conditions: $[\mathrm{Ru}]=2.3 \mathrm{mM},[\mathrm{AB}]=0.46 \mathrm{M}, 1.5 \mathrm{~mL} \mathrm{MeOH}, 60^{\circ} \mathrm{C}$. S28

Figure S26. Reaction profiles of successive $A B$ alcoholysis using $[\mathrm{Ru}(\mathrm{p}-\mathrm{Cym})(\mathrm{dhbp}) \mathrm{Cl}] \mathrm{Cl}(\mathbf{1})$. (Table 2, Entry 9). Conditions: $[\mathrm{Ru}]=2.3 \mathrm{mM},[\mathrm{AB}]=0.46 \mathrm{M}, 1.5 \mathrm{~mL} \mathrm{EtOH}, 60^{\circ} \mathrm{C}$. S29

Figure S27. Reaction profiles of successive $A B$ alcoholysis using $[\mathrm{Ru}(\mathrm{p}-\mathrm{Cym})(\mathrm{dhbp}) \mathrm{Cl}] \mathrm{Cl}(\mathbf{1})$. (Table 2, Entry 10). Conditions: $[\mathrm{Ru}]=2.3 \mathrm{mM},[\mathrm{AB}]=0.46 \mathrm{M}, 1.5 \mathrm{~mL}$ iPrOH, $60^{\circ} \mathrm{C}$.---- ------S30

Figure S28. Reaction profiles of successive $A B$ solvolysis using [Ru(p-Cym)(dhbp) Cl]Cl (1). (Table 2, Entry 11). Conditions: $[\mathrm{Ru}]=2.3 \mathrm{mM},[\mathrm{AB}]=0.46 \mathrm{M}, 1.5 \mathrm{~mL} \mathrm{H} \mathrm{O}_{2} \mathrm{O} / \mathrm{MeOH}=1 / 1,60{ }^{\circ} \mathrm{C}$-----S31

Figure S29. Reaction profiles of successive $A B$ solvolysis using $[\mathrm{Ru}(\mathrm{p}-\mathrm{Cym})(\mathrm{dhbp}) \mathrm{Cl}] \mathrm{Cl}(\mathbf{1})$. (Table 2, Entry 12). Conditions: $[\mathrm{Ru}]=2.3 \mathrm{mM},[\mathrm{AB}]=0.46 \mathrm{M}, 1.5 \mathrm{~mL} \mathrm{H} \mathrm{O}_{2} \mathrm{O} / \mathrm{MeOH}=1 / 9,60^{\circ} \mathrm{C}$.-----S32

Figure S30. Comparative reaction profiles of $A B$ solvolysis using different $\mathrm{MeOH} / \mathrm{H}_{2} \mathrm{O}$ mixtures as solvent and $[\mathrm{Ru}(\mathrm{p}-\mathrm{Cym})(\mathrm{dhbp}) \mathrm{Cl}] \mathrm{Cl}(\mathbf{1})$ as precatalyst. Conditions: $[\mathrm{Ru}]=2.3 \mathrm{mM},[\mathrm{AB}]=0.46$ $\mathrm{M}, 1.5 \mathrm{~mL}$ solvent, $60^{\circ} \mathrm{C}$. $-\mathrm{S33}$

Figure S31. Reaction profiles of successive $A B$ solvolysis using $[R u(p-C y m)(d h b p) C l] C l(1)$. (Table

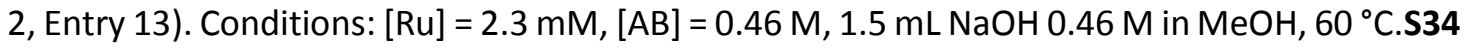


Figure S32 Reaction profiles of successive AB hydrolysis using [Ru(p-Cym)(dhbp)Cl]Cl (1). (Table 2, Entry 14). Conditions: $[\mathrm{Ru}]=2.3 \mathrm{mM},[\mathrm{AB}]=0.46 \mathrm{M}, 100 \mathrm{mg} \mathrm{NaCl}$-modified molecular sieves, $1.5 \mathrm{~mL} \mathrm{H} \mathrm{H}_{2}, 60^{\circ} \mathrm{C}$.S34

Figure S33. Reaction profiles of successive $A B$ hydrolysis using $[\mathrm{Ru}(\mathrm{p}-\mathrm{Cym})(\mathrm{dhbp}) \mathrm{Cl}] \mathrm{Cl}(\mathbf{1})$.

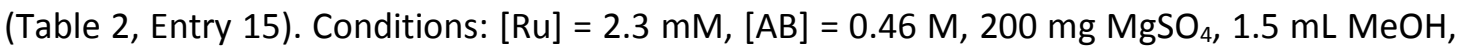
$60{ }^{\circ} \mathrm{C}$.

S35

Figure S34. Reaction profiles of: TOP: $\mathrm{HMe}_{2} \mathrm{~N} \cdot \mathrm{BH}_{3}$ (DMAB) hydrolysis using $[\mathrm{Ru}(\mathrm{p}-$ Cym) (dhbp)Cl]Cl (1). (Table S1, Entry 1). Conditions: $[\mathrm{Ru}]=2.3 \mathrm{mM}$, [DMAB] $=0.46 \mathrm{M}, 1.5 \mathrm{~mL}$ $\mathrm{H}_{2} \mathrm{O}, 60^{\circ} \mathrm{C}$. BOTTOM: Comparison of DMAB and $\mathrm{AB}$ hydrolysis using $[\mathrm{Ru}(\mathrm{p}-\mathrm{Cym})(\mathrm{dhbp}) \mathrm{Cl}] \mathrm{Cl}(\mathbf{1})$ as precatalyst.

Figure S35. Reaction profile of DMAB alcoholysis using $[\mathrm{Ru}(\mathrm{p}-\mathrm{Cym})(\mathrm{dhbp}) \mathrm{Cl}] \mathrm{Cl}$ (1). (Table $\mathrm{S} 1$, Entry 2). Conditions: $[\mathrm{Ru}]=2.3 \mathrm{mM},[\mathrm{DMAB}]=0.46 \mathrm{M}, 1.5 \mathrm{~mL} \mathrm{MeOH}, 60^{\circ} \mathrm{C}$. S36

Figure S36. Reaction profile of DMAB hydrolysis using $[\mathrm{Ru}(\mathrm{p}-\mathrm{Cym})(\mathrm{dhbp}) \mathrm{Cl}] \mathrm{Cl}(\mathbf{1})$. (Table $\mathrm{S} 1$, Entry 3). Conditions: $[\mathrm{Ru}]=2.3 \mathrm{mM},\left[\mathrm{HMe}_{2} \mathrm{~N} \cdot \mathrm{BH}_{3}\right]=[\mathrm{NaOH}]=0.46 \mathrm{M}, 1.5 \mathrm{~mL} \mathrm{H} \mathrm{H}_{2}, 60^{\circ} \mathrm{C} .--\mathrm{S} 37$

Figure S37. Reaction profile of DMAB alcoholysis using $[\mathrm{Ru}(\mathrm{p}-\mathrm{Cym})(\mathrm{dhbp}) \mathrm{Cl}] \mathrm{Cl}(\mathbf{1})$. (Table $\mathrm{S} 1$, Entry 4). Conditions: $[\mathrm{Ru}]=2.3 \mathrm{mM},[\mathrm{DMAB}]=[\mathrm{NaOH}]=0.46 \mathrm{M}, 1.5 \mathrm{~mL} \mathrm{MeOH}, 60^{\circ} \mathrm{C}$.------ $\mathrm{S37}$

Table S1. Catalytic results. DMAB solvolysis using precatalysts 1. S37

In situ NMR characterizations.

Scheme S1. Reactivity scheme used for the in situ characterizations in $\mathrm{D}_{2} \mathrm{O}$. S38

Figure S38. ${ }^{1} \mathrm{H}$ NMR spectra $(300 \mathrm{MHz}) .3 .89 \mathrm{mg}$ of $[\mathrm{Ru}(\mathrm{p}-\mathrm{Cym})(\mathrm{dhbp}) \mathrm{Cl}] \mathrm{Cl}(\mathbf{1})$ in $0.5 \mathrm{~mL}$ of $\mathrm{D}_{2} \mathrm{O}$.S39

Figure S39. COSY NMR spectra (300 MHz). $3.89 \mathrm{mg}$ of $[\mathrm{Ru}(\mathrm{p}-\mathrm{Cym})(\mathrm{dhbp}) \mathrm{Cl}] \mathrm{Cl}(\mathbf{1})$ in $0.5 \mathrm{~mL}$ of $\mathrm{D}_{2} \mathrm{O}$. $-\mathrm{S} 40$

Figures S40-S44. In situ formation and characterization of $[\mathrm{Ru}(\mathrm{p}-\mathrm{Cym})(\mathrm{dhbp}) \mathrm{Cl}]^{+}\left(\mathrm{D}_{2} \mathrm{O}\right)$------S41

Figures S45-S49. In situ formation and characterization of $\left[R u(p-C y m)(d h b p)\left(D_{2} O\right)\right]^{2+}\left(D_{2} O\right) . S 46$

Figures S50-S54. In situ formation and characterization of $\left[\mathrm{Ru}(\mathrm{p}-\mathrm{Cym})(\mathrm{dhbp}-2 \mathrm{H})\left(\mathrm{ND}_{3}\right)\right]^{2+}$ $\left(\mathrm{D}_{2} \mathrm{O}\right)$. $-\mathrm{S} 51$

Figures S55-S58. In situ formation and characterization of $[\mathrm{Ru}(\mathrm{p}-\mathrm{Cym})(\mathrm{dhbp}-2 \mathrm{H})(\mathrm{OD})]^{-}\left(\mathrm{D}_{2} \mathrm{O}\right)$. S56

Scheme S2. Reactivity scheme used for the in situ characterizations in $\mathrm{CD}_{3} \mathrm{OD}$. $-560$

Figures S59-S62. In situ formation and characterization of $[\mathrm{Ru}(\mathrm{p}-\mathrm{Cym})(\mathrm{dhbp}) \mathrm{Cl}]^{+}\left(\mathrm{CD}_{3} \mathrm{OD}\right)$.-S61

Figures S63-S67. In situ formation and characterization of $\left[\mathrm{Ru}(\mathrm{p}-\mathrm{Cym})(\mathrm{dhbp}-2 \mathrm{H})\left(\mathrm{ND}_{3}\right)\right]^{2+}$ $\left(\mathrm{CD}_{3} \mathrm{O}\right)$.

Figures S68-S74 In situ catalytic experiments, and characterization of [Ru(p-Cym)(dhbp$2 \mathrm{H}) \mathrm{H})]^{-}$ S70

Table S2. ${ }^{1} \mathrm{H}$ NMR chemical shift data $(\mathrm{ppm})$ of $[\mathrm{Ru}(\mathrm{pCym})(\mathrm{dhbp}) \mathrm{L}]^{\mathrm{nt}}$ and $[\mathrm{Ru}(\mathrm{pCym})(\mathrm{dhbp}-$ $2 \mathrm{H}) \mathrm{L}]^{\text {n- }}$ generated in situ. 
In situ NMR catalytic experiments.

Figure S75. In situ ${ }^{1} \mathrm{H}$ NMR experiment (300 MHz, $\left.\mathrm{D}_{2} \mathrm{O}\right)$. A $0.75 \mathrm{mg}$ of $[\mathrm{Ru}(\mathrm{p}-\mathrm{Cym})(\mathrm{dhbp}) \mathrm{Cl}] \mathrm{Cl}(\mathbf{1})$ in $0.5 \mathrm{~mL}$ of $\mathrm{D}_{2} \mathrm{O}$

Figure S76-S77. In situ ${ }^{1} \mathrm{H}$ NMR experiment (300 MHz, CD $\mathrm{OD}$ ). A $0.75 \mathrm{mg}$ of $[\mathrm{Ru}(\mathrm{p}-$ $\mathrm{Cym})(\mathrm{dhbp}) \mathrm{Cl} \mathrm{Cl}(\mathbf{1})$ in $0.5 \mathrm{~mL}$ of $\mathrm{CD}_{3} \mathrm{OD}$.--

Figure S78. In situ NMR experiment. $0.75 \mathrm{mg}$ of $[\mathrm{Ru}(\mathrm{p}-\mathrm{Cym})(\mathrm{dhbp}) \mathrm{Cl}] \mathrm{Cl}(\mathbf{1})$ in $0.5 \mathrm{~mL}$ of $\mathrm{NaOD} / \mathrm{D}_{2} \mathrm{O} 0.001 \mathrm{M}$.

\section{NMR spectra at the end of the catalytic processes.}

Figure S79. ${ }^{1} \mathrm{H}$ NMR spectra (300 MHz). A. $3.89 \mathrm{mg}$ of $[\mathrm{Ru}(\mathrm{p}-\mathrm{Cym})(\mathrm{dhbp}) \mathrm{Cl}] \mathrm{Cl}(\mathbf{1})$ in $0.5 \mathrm{~mL}$ of $\mathrm{D}_{2} \mathrm{O}+20 \mu \mathrm{L} \mathrm{NH}$ aq. $20 \% \mathrm{v} / \mathrm{v}$. B. Reaction solution at the end of a catalytic run using $[\mathrm{Ru}(\mathrm{p}-$ Cym) (dhbp) Cl]Cl (1) as catalyst in $\mathrm{H}_{2} \mathrm{O}$ (internal capillary of $\mathrm{D}_{2} \mathrm{O}$ ). C. Reaction solution at the end of a catalytic run using $\left[\mathrm{Ru}(\mathrm{p}-\mathrm{Cym})(\mathrm{dhbp})\left(\mathrm{H}_{2} \mathrm{O}\right)\right](\mathrm{OTf})_{2}(2)$ as catalyst in $\mathrm{H}_{2} \mathrm{O}$ (internal capillary of $\left.\mathrm{D}_{2} \mathrm{O}\right)$..

$-582$

Figure S80. ${ }^{1} \mathrm{H}$ NMR spectra (300 MHz). A $3.85 \mathrm{mg}$ of $[\mathrm{Ru}(\mathrm{p}-\mathrm{Cym})(\mathrm{dhbp}) \mathrm{Cl}] \mathrm{Cl}(\mathbf{1})$ in $0.5 \mathrm{~mL}$ of $\mathrm{CD}_{3} \mathrm{OD}+20 \mu \mathrm{L} \mathrm{NH}$ aq. $20 \% \mathrm{v} / \mathrm{v}$. B Reaction solution at the end of a catalytic run using [Ru(pCym) (dhbp) Cl] Cl (1) as catalyst in $\mathrm{CH}_{3} \mathrm{OH}$ (internal capillary of $\mathrm{D}_{2} \mathrm{O}$ ).

$-\mathrm{S} 82$

Figure S81. ${ }^{11} \mathrm{~B}$ NMR spectra (128 MHz) of reaction solutions at the end of a catalytic run using $[\mathrm{Ru}(\mathrm{p}-\mathrm{Cym})(\mathrm{dhbp}) \mathrm{Cl}] \mathrm{Cl}(\mathbf{1})$ as catalyst in different solvents. ${ }^{11} \mathrm{~B}$ NMR spectra at the end of the catalytic reactions show broad bands in the region 2-19 ppm which are characteristic of polyborate ions in equilibrium in solution, as described before by other authors. ${ }^{[1]}$ $-S 83$

\section{Mechanistic considerations and DFT calculations.}

Scheme S3. Ligands exchange processes and estimated $\Delta \mathrm{G}$, according to DFT calculations.--S84

Scheme S4. Alternative reaction pathway for the hydrogen-generation step with dhbpprotonated system. S85

Table S3. Summary of the energetic values (Hartree) of the calculated species $--585$

\section{Homogeneity tests.}

Figure S82. Reaction profiles of $A B$ solvolysis using $[R u(p-C y m)(\mathrm{dhbp}) \mathrm{Cl}] \mathrm{Cl}(\mathbf{1})$. (Table 2, Entry 4). Conditions: blue line: $[\mathrm{Ru}]=2.3 \mathrm{mM},[\mathrm{AB}]=0.46 \mathrm{M}, 1.5 \mathrm{~mL}$ (HEPES buffer $1 \mathrm{M}$ in $\mathrm{H}_{2} \mathrm{O}, \mathrm{pH}$ 7.0), $60{ }^{\circ} \mathrm{C}$. red line: $[R u]=2.3 \mathrm{mM},[A B]=0.46 \mathrm{M}, \mathrm{Hg}$ (1000 equiv. per $\mathrm{Ru}$ ), $1.5 \mathrm{~mL}$ (HEPES buffer $1 \mathrm{M}$ in $\mathrm{H}_{2} \mathrm{O}$, pH 7.0), $60^{\circ} \mathrm{C}$. S86

Figure S83. Reaction profiles of $A B$ solvolysis using $[\mathrm{Ru}(\mathrm{p}-\mathrm{Cym})(\mathrm{dhbp}) \mathrm{Cl}] \mathrm{Cl}(\mathbf{1})$. (Table 2, Entry 4). Conditions: blue line: $[\mathrm{Ru}]=2.3 \mathrm{mM},[\mathrm{AB}]=0.46 \mathrm{M}, 1.5 \mathrm{~mL} \mathrm{H} \mathrm{H}_{2}, 60^{\circ} \mathrm{C}$. red line: $[\mathrm{Ru}]=2.3$ $\mathrm{mM},[\mathrm{AB}]=0.46 \mathrm{M}, \mathrm{Hg}$ (1000 equiv. per $\mathrm{Ru}), 1.5 \mathrm{~mL} \mathrm{H} \mathrm{H}_{2}, 60^{\circ} \mathrm{C}$ $-587$ 
a

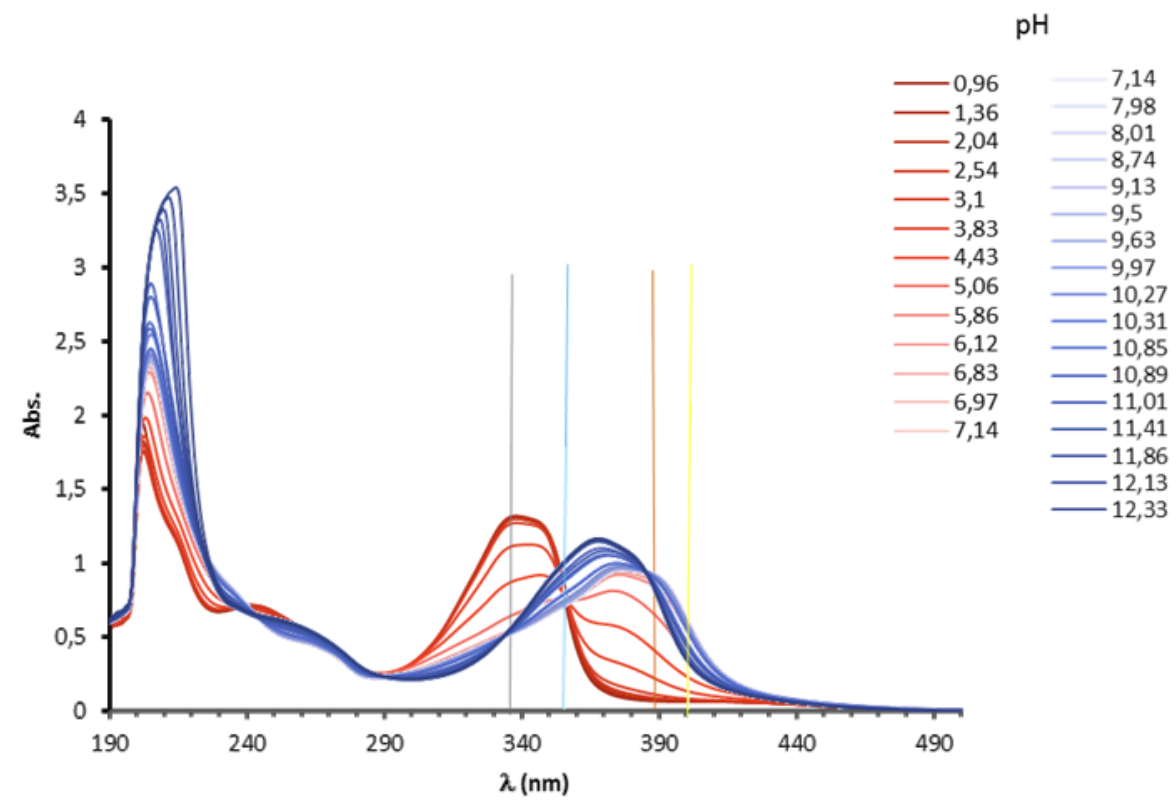

b

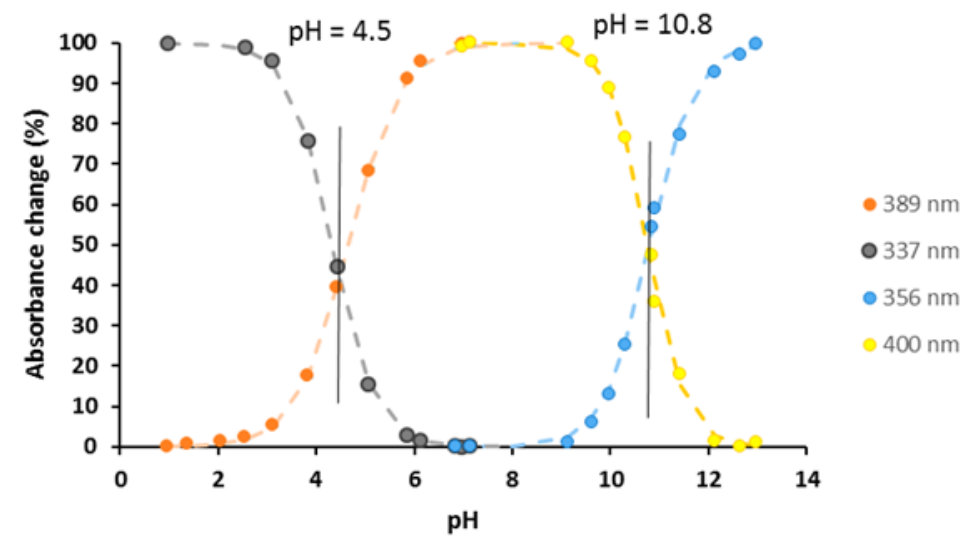

Figure S1. a) UV-vis absorption spectra of $\left[\mathrm{Ru}(\mathrm{p}-\mathrm{Cym})(\mathrm{dhbp})\left(\mathrm{H}_{2} \mathrm{O}\right)\right](\mathrm{OTf})_{2}(2)$ in a pH titration in $\mathrm{H}_{2} \mathrm{O}$. b) Absorbance changes at single wavelengths as a function of $\mathrm{pH}$. Botzmann fits used to calculate the corresponding $\mathrm{pH}$ inflexion points are plotted using discontinuous lines. The $\mathrm{pH}$ was adjusted by mixing $0.8 \cdot 10^{-4} \mathrm{M}$ stock solutions of complex 2 in $0.1 \mathrm{M} \mathrm{HCl}$ and $0.1 \mathrm{M} \mathrm{NaOH}$, respectively. 
a

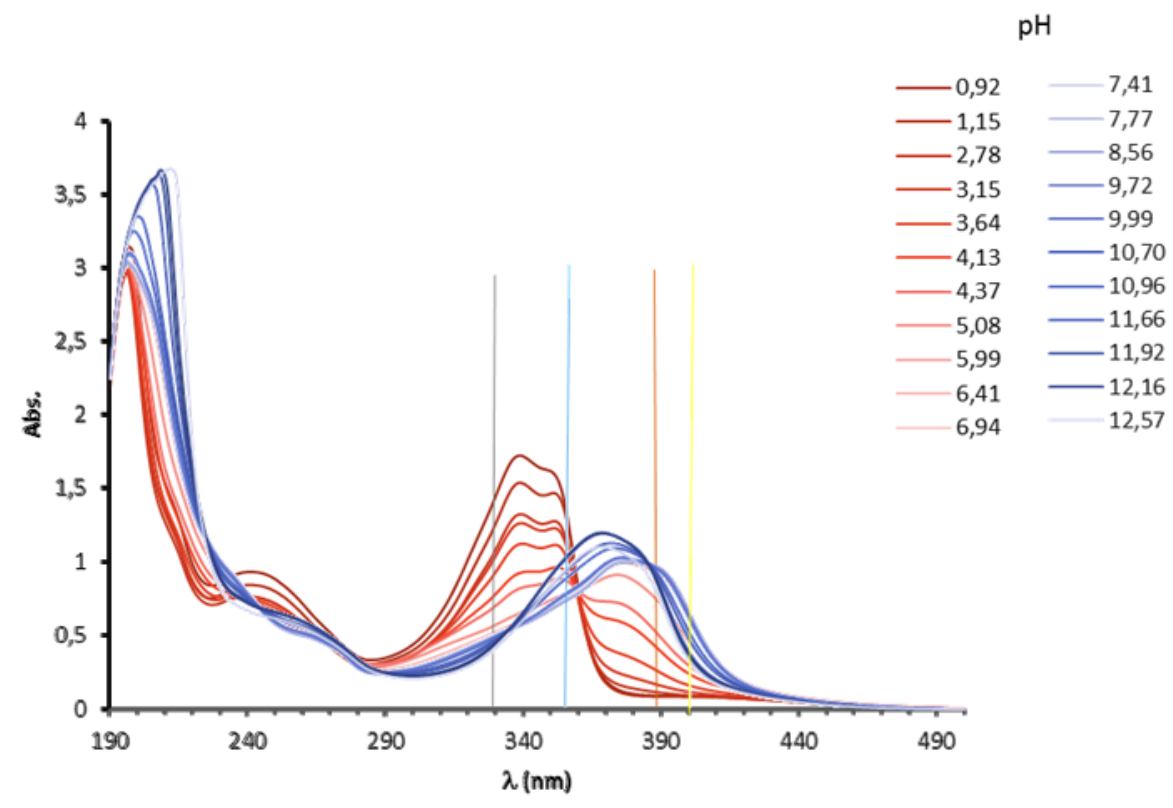

b

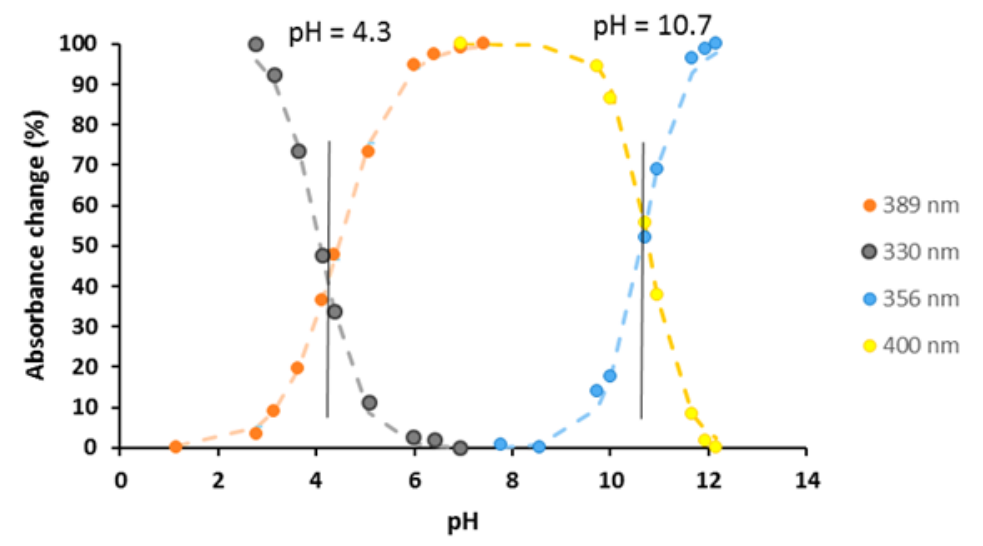

Figure S2. a) UV-vis absorption spectra of $[\mathrm{Ru}(\mathrm{p}-\mathrm{Cym})(\mathrm{dhbp}) \mathrm{Cl}] \mathrm{Cl}(\mathbf{1})$ in a $\mathrm{pH}$ titration in $\mathrm{H}_{2} \mathrm{O}$. b) Absorbance changes at single wavelengths as a function of $\mathrm{pH}$. Botzmann fits used to calculate the corresponding $\mathrm{pH}$ inflexion points are plotted using discontinuous lines. The $\mathrm{pH}$ was adjusted by mixing $0.1 \cdot 10^{-3} \mathrm{M}$ stock solutions of complex 1 in $0.02 \mathrm{M} \mathrm{H}_{2} \mathrm{SO}_{4}$ and $0.1 \mathrm{M} \mathrm{NaOH}$, respectively. 
a

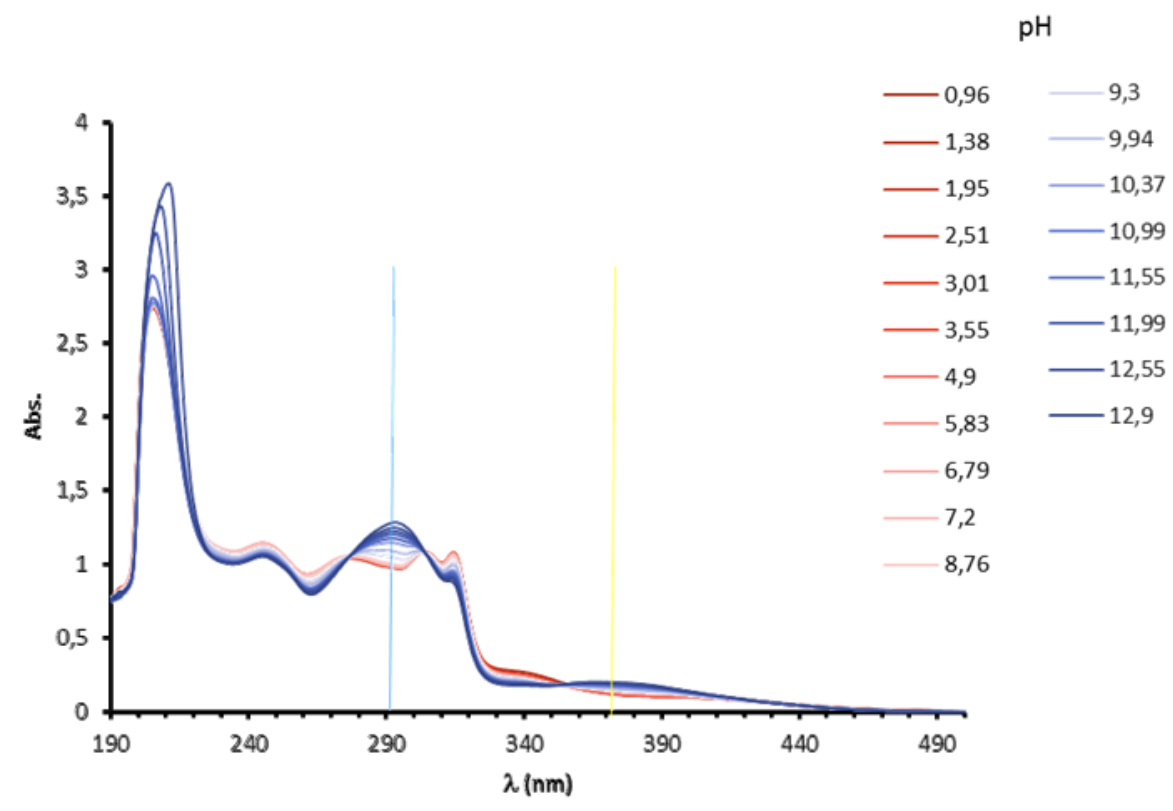

b

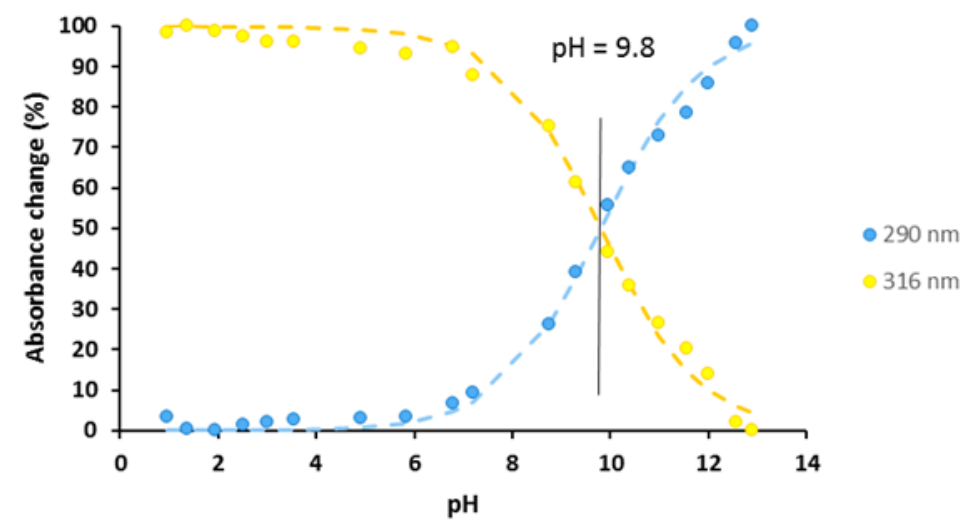

Figure S3. a) UV-vis absorption spectra of $[\mathrm{Ru}(\mathrm{p}-\mathrm{Cym})(\mathrm{bipy}) \mathrm{C}] \mathrm{Cl}(\mathbf{V})$ in a $\mathrm{pH}$ titration in $\mathrm{H}_{2} \mathrm{O}$. b) Absorbance changes at single wavelengths as a function of $\mathrm{pH}$. Botzmann fits used to calculate the corresponding $\mathrm{pH}$ inflexion points are plotted using discontinuous lines. The $\mathrm{pH}$ was adjusted by mixing $0.1 \cdot 10^{-3} \mathrm{M}$ stock solutions of complex $\mathrm{V}$ in $0.1 \mathrm{M} \mathrm{HCl}$ and $0.1 \mathrm{M} \mathrm{NaOH}$, respectively. 

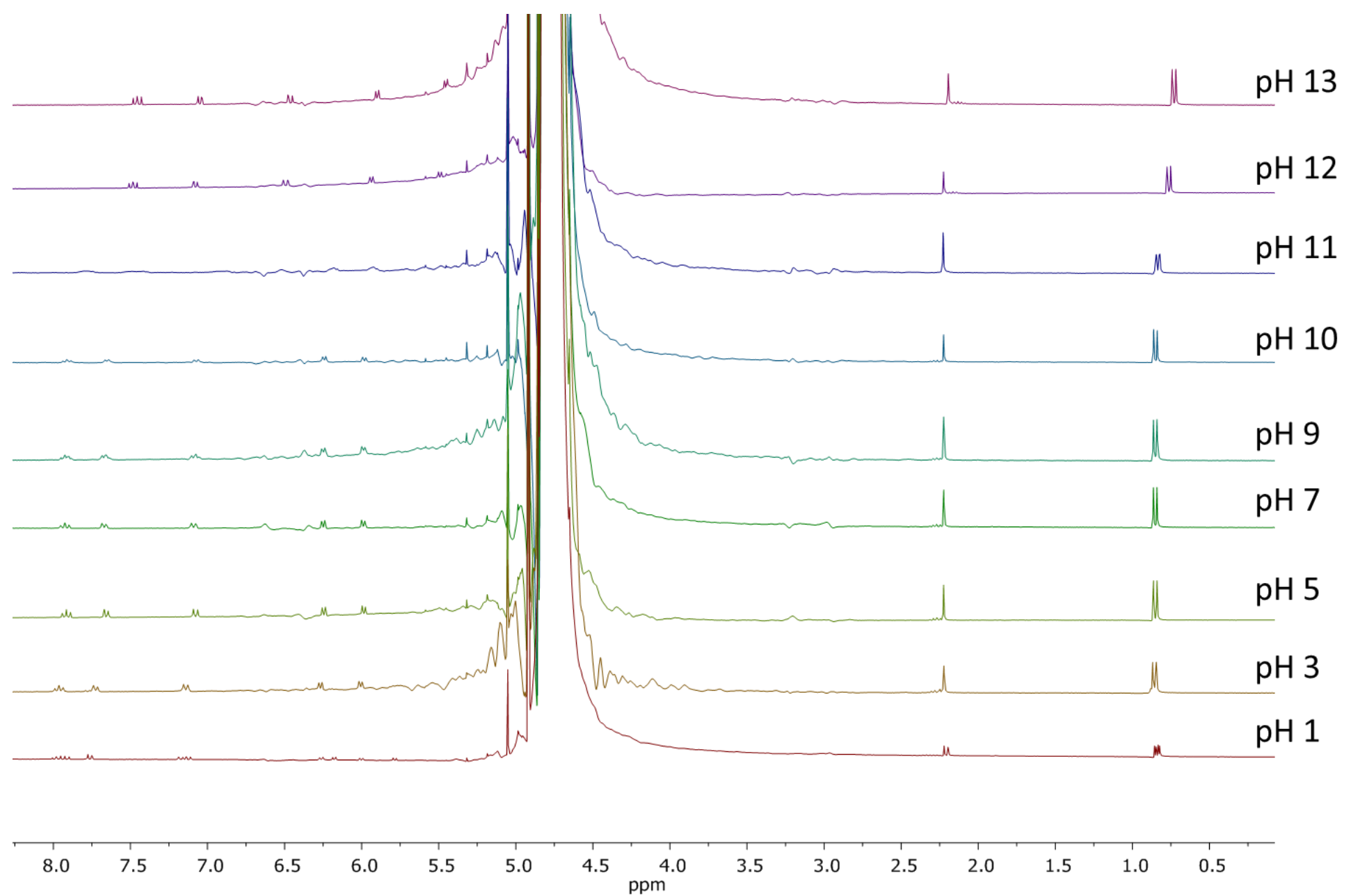

Figure S4. ${ }^{1} \mathrm{H}$ NMR spectra $(300 \mathrm{MHz})$ of $\left[\mathrm{Ru}(\mathrm{p}-\mathrm{Cym})(\mathrm{dhbp})\left(\mathrm{H}_{2} \mathrm{O}\right)\right](\mathrm{OTf})_{2}(2)$ in a pH titration in $\mathrm{H}_{2} \mathrm{O}$ (internal capillary of $\mathrm{D}_{2} \mathrm{O}$ ). $0.50 \mathrm{mg}$ of $[\mathrm{Ru}(\mathrm{p}$ Cym)(dhbp) $\left.\left(\mathrm{H}_{2} \mathrm{O}\right)\right](\mathrm{OTf})_{2}(2)$ in $0.5 \mathrm{~mL}$ of $\mathrm{D}_{2} \mathrm{O}$ at the indicated $\mathrm{pH}$ adjusted with $0.1 \mathrm{M}$ stock solutions of $\mathrm{HCl}$ and $\mathrm{NaOH}$. 

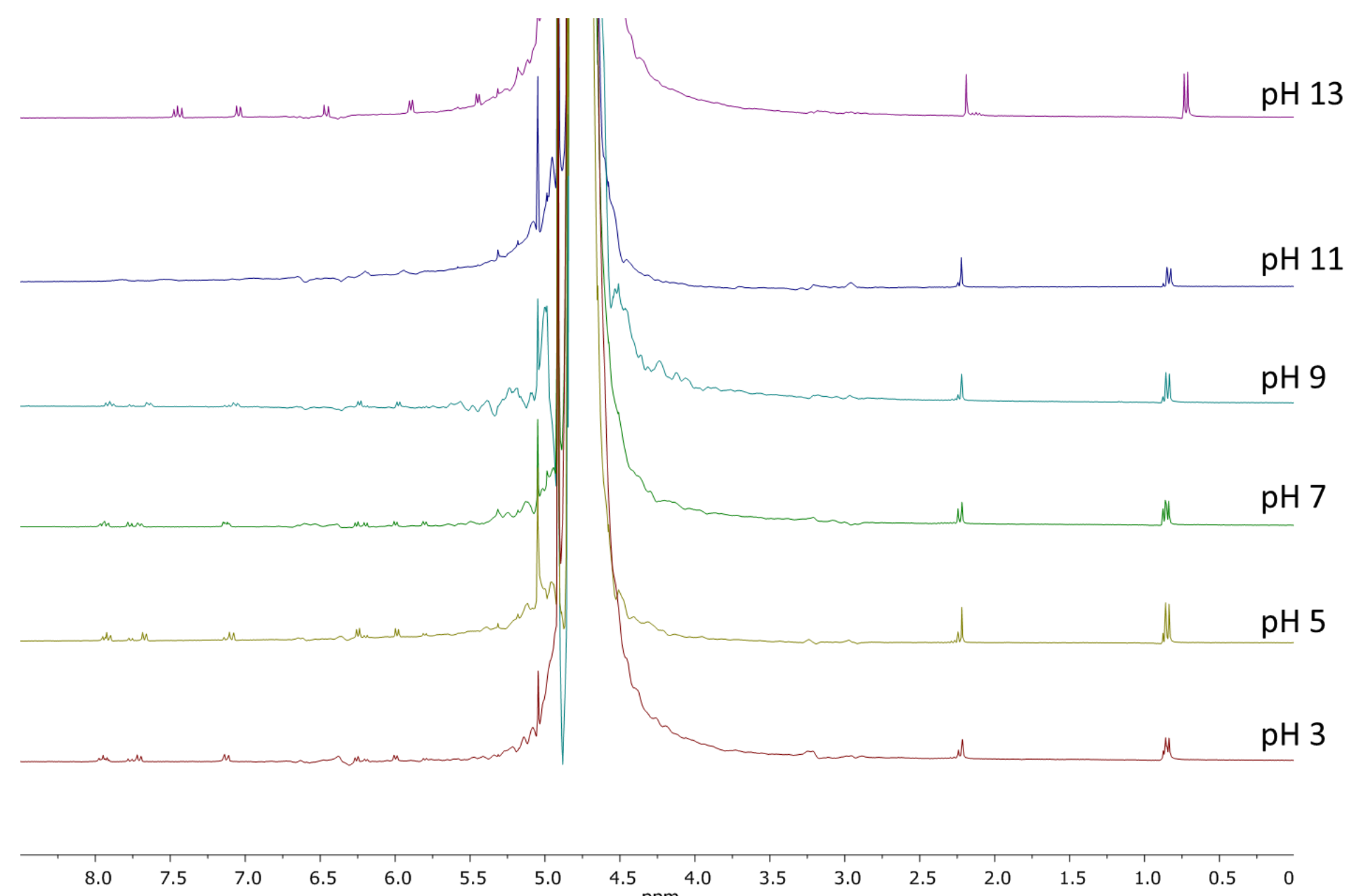

Figure S5. ${ }^{1} \mathrm{H}$ NMR spectra $(300 \mathrm{MHz})$ of $[\mathrm{Ru}(\mathrm{p}-\mathrm{Cym})(\mathrm{dhbp}) \mathrm{Cl}] \mathrm{Cl}(\mathbf{1})$ in a pH titration in $\mathrm{H}_{2} \mathrm{O}$ (internal capillary of $\left.\mathrm{D}_{2} \mathrm{O}\right) .0 .57 \mathrm{mg}$ of $[\mathrm{Ru}(\mathrm{p}-\mathrm{Cym})(\mathrm{dhbp}) \mathrm{Cl}] \mathrm{Cl}(\mathbf{1})$ in $0.5 \mathrm{~mL}$ of $\mathrm{D}_{2} \mathrm{O}$ at the indicated $\mathrm{pH}$ adjusted with $0.1 \mathrm{M}$ stock solutions of $\mathrm{HCl}$ and $\mathrm{NaOH}$. 

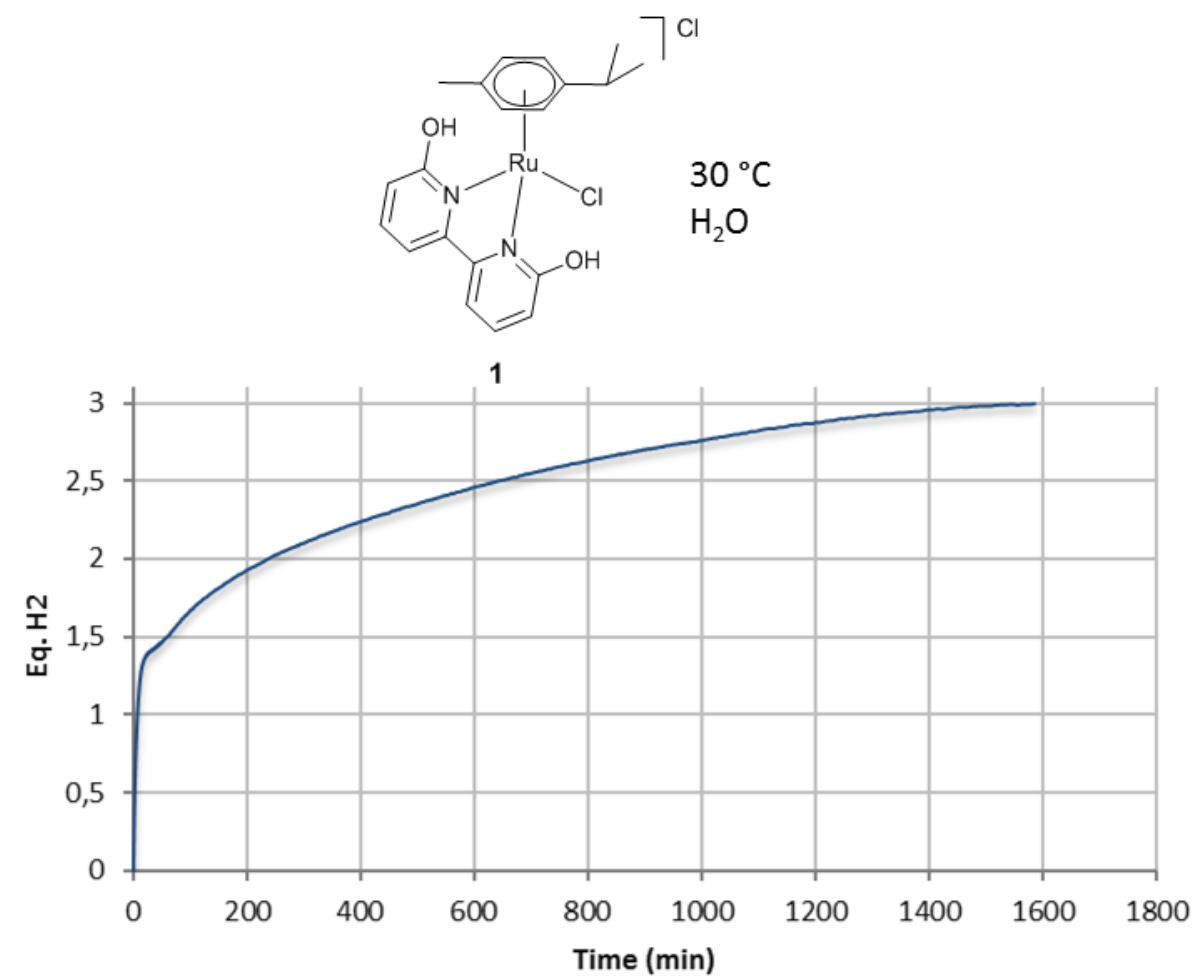

Figure S6. Reaction profile of $A B$ hydrolysis using $[\mathrm{Ru}(\mathrm{p}-\mathrm{Cym})(\mathrm{dhbp}) \mathrm{Cl}] \mathrm{Cl}(\mathbf{1})$ as precatalyst. (Table 1, Entry 1). Conditions: $[\mathrm{Ru}]=2.3 \mathrm{mM},[\mathrm{AB}]=0.46 \mathrm{M}, 1.5 \mathrm{~mL} \mathrm{H}{ }_{2} \mathrm{O}, 30^{\circ} \mathrm{C}$.
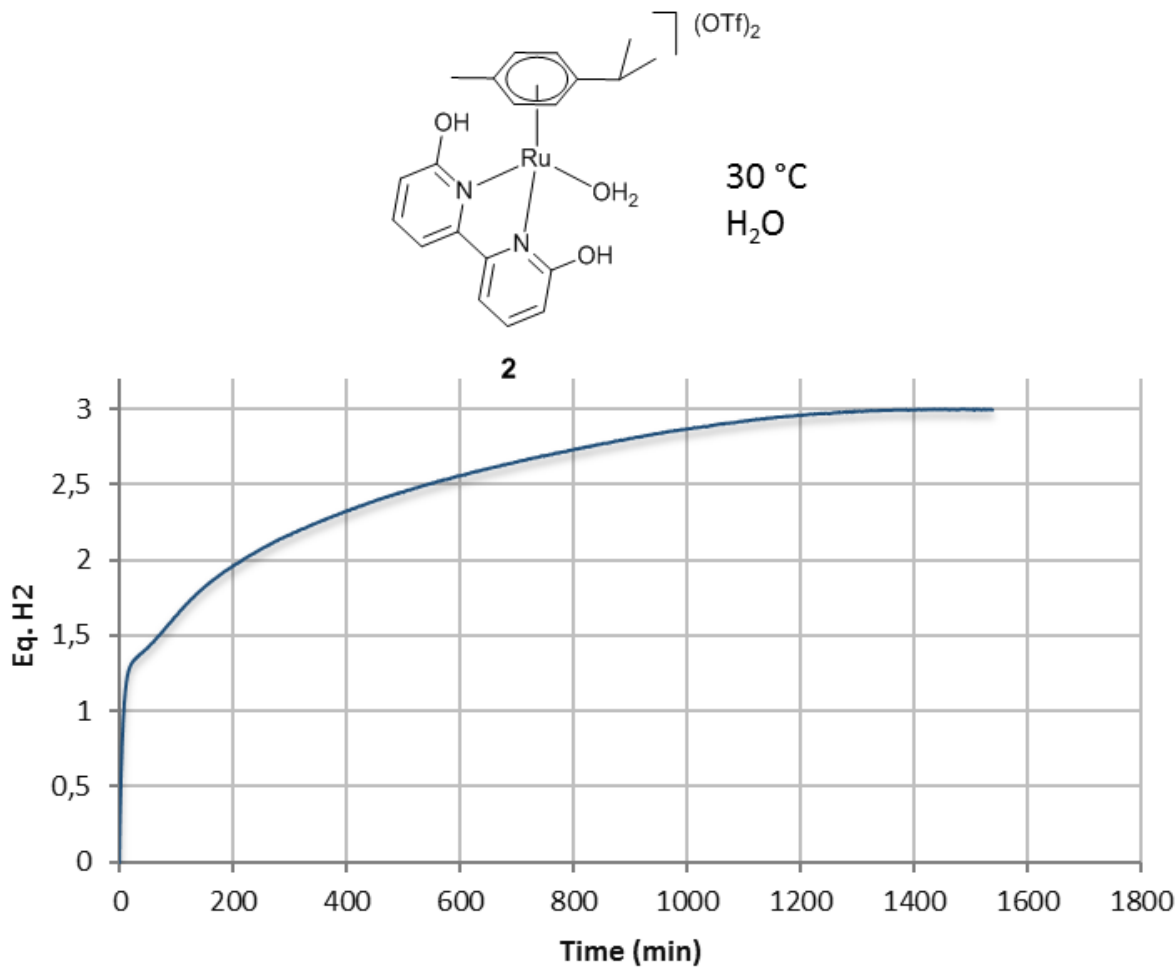

Figure S7. Reaction profile of $A B$ hydrolysis using $\left[\mathrm{Ru}(\mathrm{p}-\mathrm{Cym})(\mathrm{dhbp})\left(\mathrm{H}_{2} \mathrm{O}\right)\right](\mathrm{OTf})_{2}$ (2) as precatalyst. (Table 1, Entry 2). Conditions: $[\mathrm{Ru}]=2.3 \mathrm{mM},[\mathrm{AB}]=0.46 \mathrm{M}, 1.5 \mathrm{~mL} \mathrm{H} \mathrm{H}_{2} \mathrm{O}, 30^{\circ} \mathrm{C}$ 


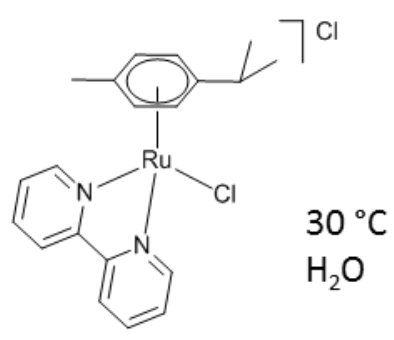

4

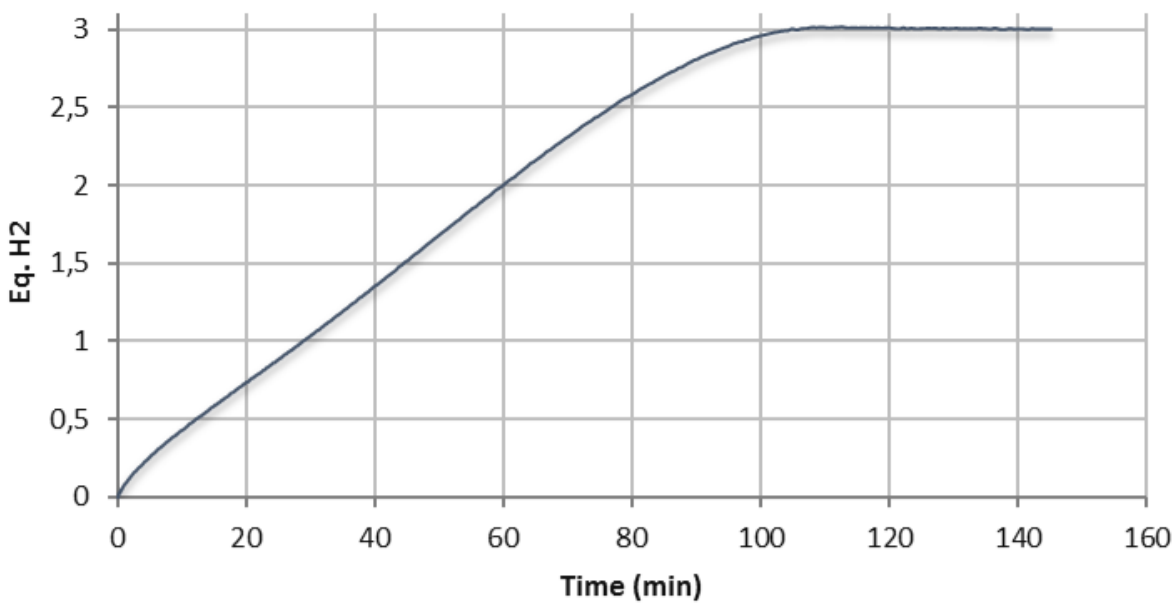

Figure S8. Reaction profile of $A B$ hydrolysis using $[\mathrm{Ru}(\mathrm{p}-\mathrm{Cym})(\mathrm{bipy}) \mathrm{Cl}] \mathrm{Cl}(\mathrm{V})$ as precatalyst. (Table 1, Entry 3). Conditions: $[R u]=2.3 \mathrm{mM},[A B]=0.46 \mathrm{M}, 1.5 \mathrm{~mL} \mathrm{H}{ }_{2} \mathrm{O}, 30^{\circ} \mathrm{C}$.

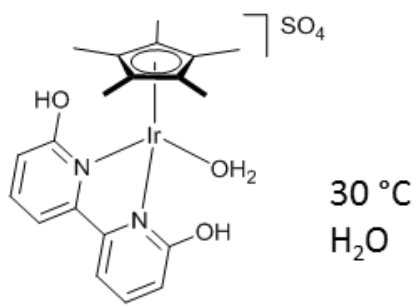

3

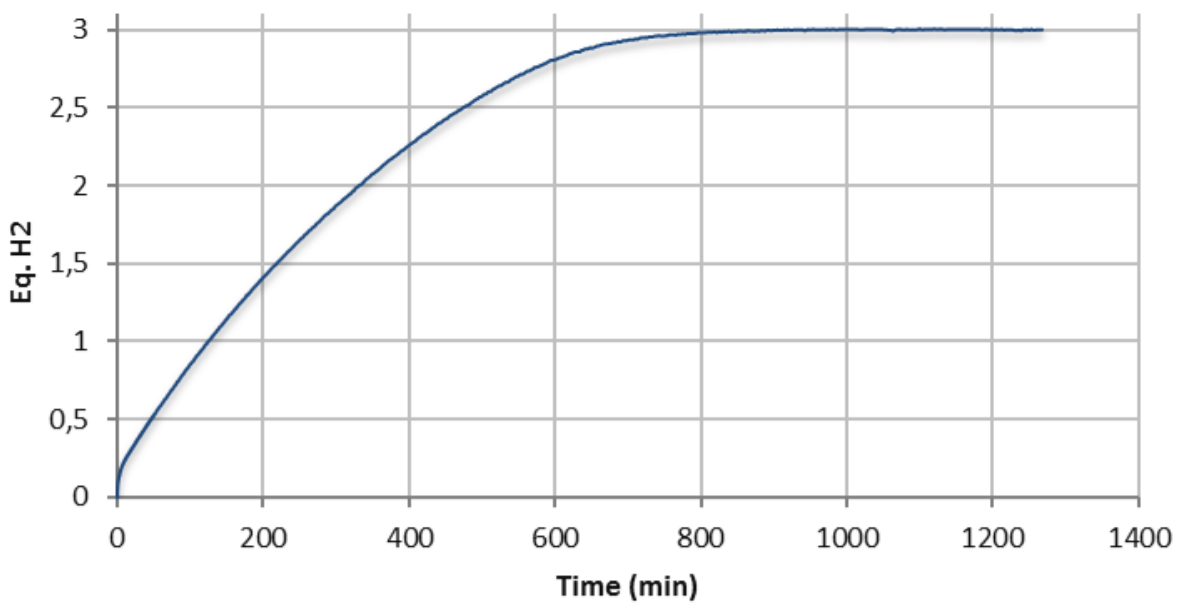

Figure S9. Reaction profile of $A B$ hydrolysis using [IrCp*(dhbp)Cl]Cl (Himeda's catalyst) (VI) as precatalyst. (Table 1, Entry 4). Conditions: $[\mathrm{Ir}]=2.3 \mathrm{mM},[\mathrm{AB}]=0.46 \mathrm{M}, 1.5 \mathrm{~mL} \mathrm{H} \mathrm{H}_{2} \mathrm{O}, 30^{\circ} \mathrm{C}$. 

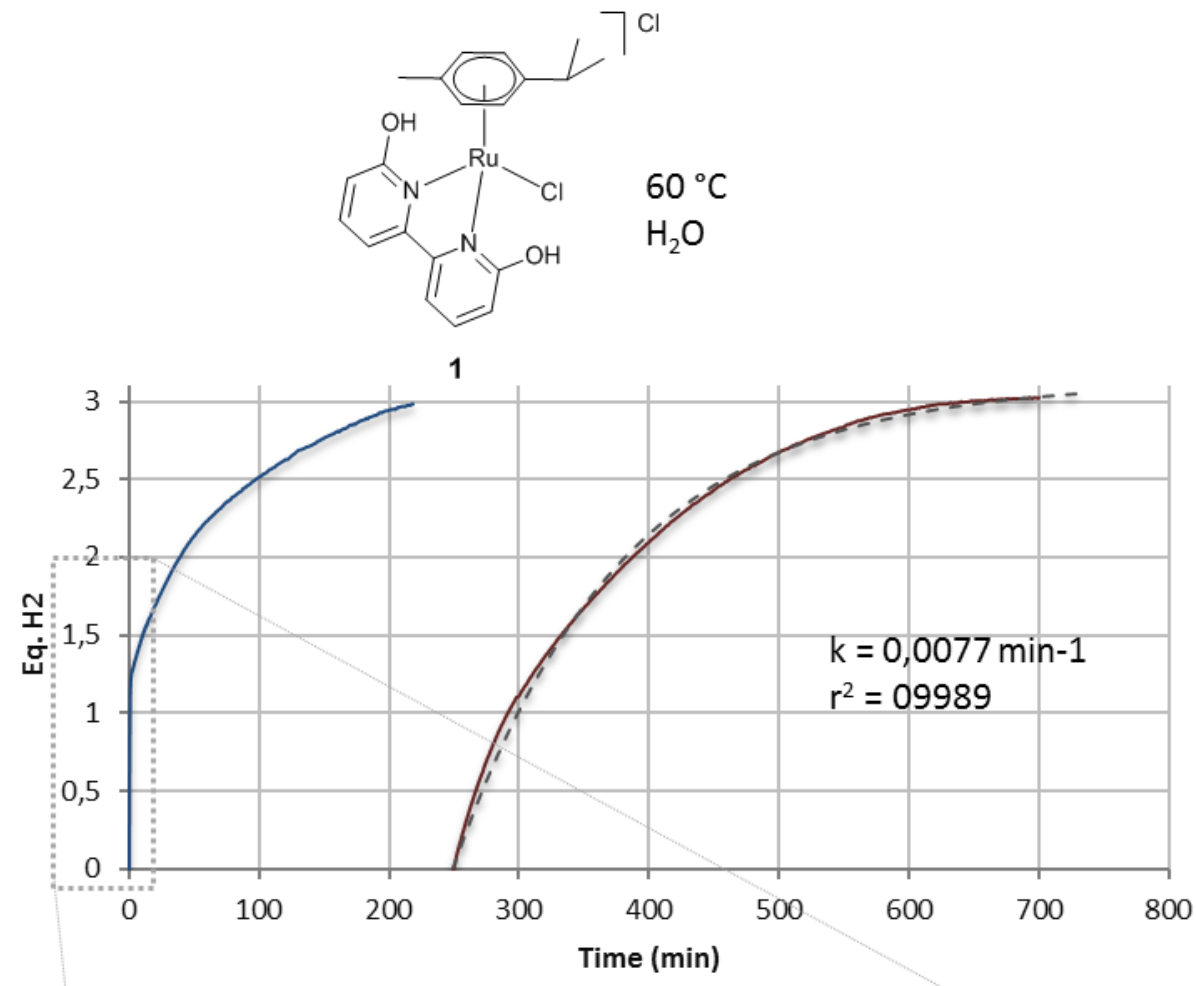

- Series2 - 2nd addition $\quad---1$ st order fit

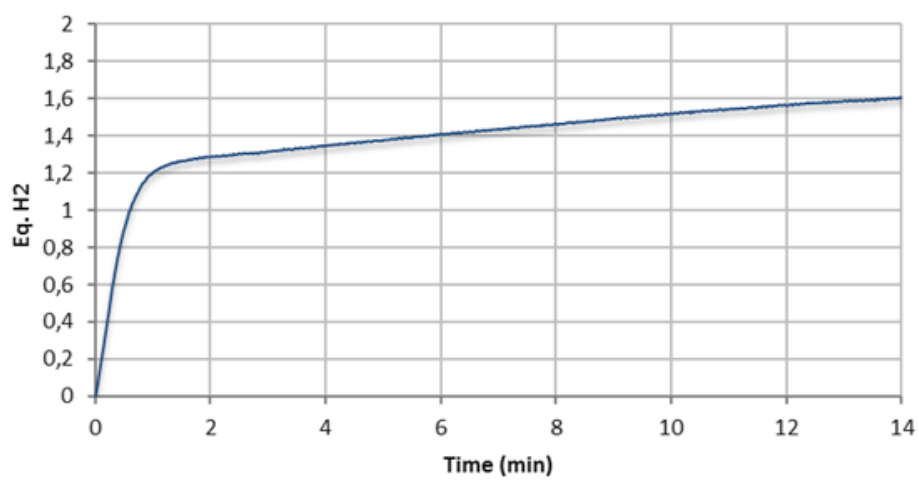

Figure S10. Reaction profiles of successive $A B$ hydrolysis using $[\mathrm{Ru}(\mathrm{p}-\mathrm{Cym})(\mathrm{dhbp}) \mathrm{Cl}] \mathrm{Cl}(\mathbf{1})$ as precatalyst. (Table 1, Entry 5). First-order kinetic adjustment for the second addition shown. Conditions: $[\mathrm{Ru}]=2.3 \mathrm{mM},[\mathrm{AB}]=0.46 \mathrm{M}, 1.5 \mathrm{~mL} \mathrm{H}_{2} \mathrm{O}, 60^{\circ} \mathrm{C}$. Additional $21.3 \mathrm{mg}$ of $A B$ for the consecutive reaction. 


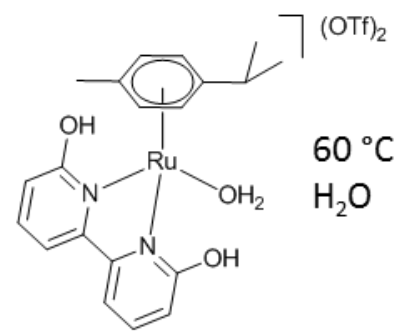

2
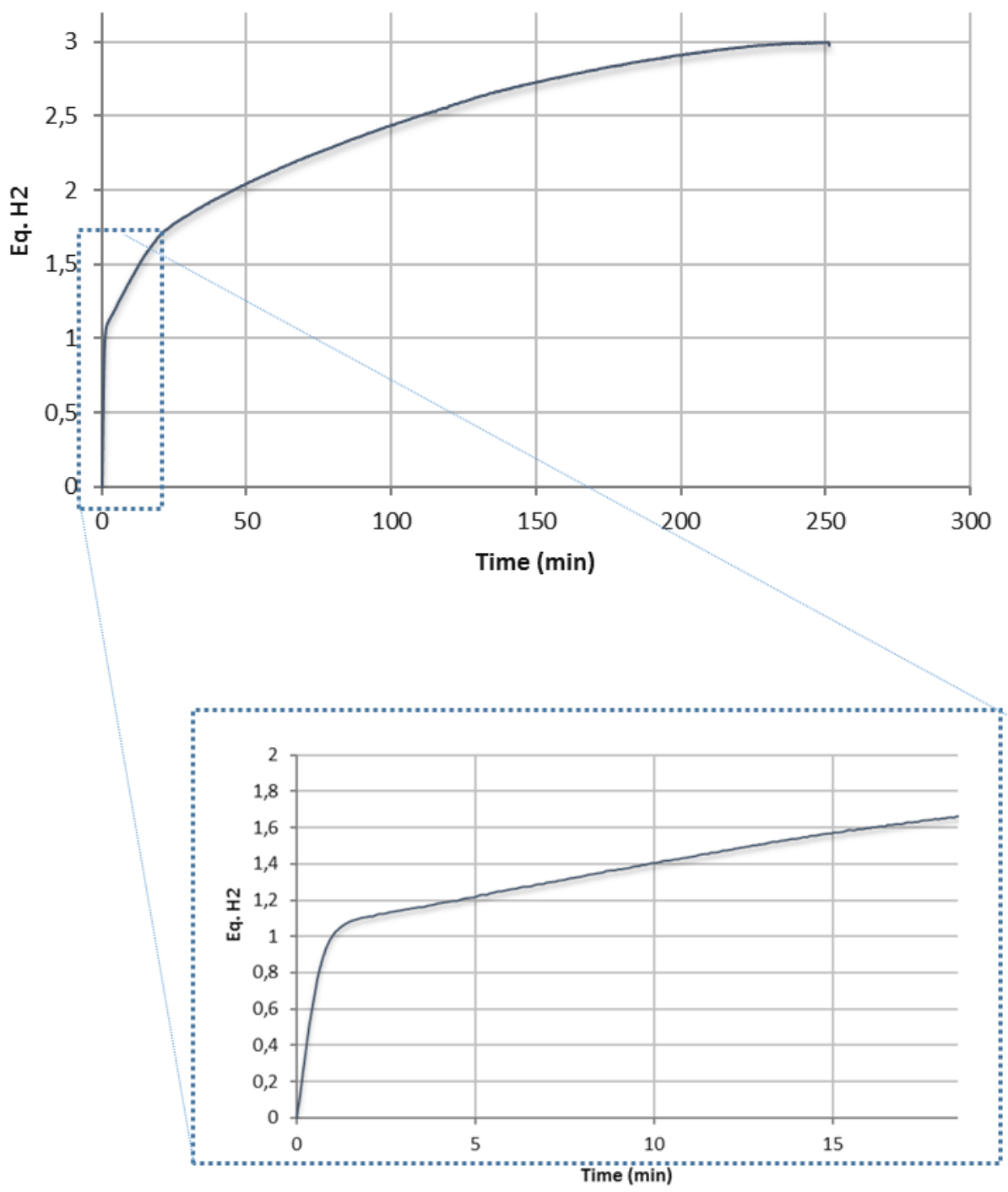

Figure S11. Reaction profile of $A B$ hydrolysis using $\left[\mathrm{Ru}(\mathrm{p}-\mathrm{Cym})(\mathrm{dhbp})\left(\mathrm{H}_{2} \mathrm{O}\right)\right](\mathrm{OTf})_{2}$ (2) as precatalyst. (Table 1 entry 6). Conditions: $[R u]=2.3 \mathrm{mM},[A B]=0.46 \mathrm{M}, 1.5 \mathrm{~mL} \mathrm{H} \mathrm{H}_{2}, 60^{\circ} \mathrm{C}$. 

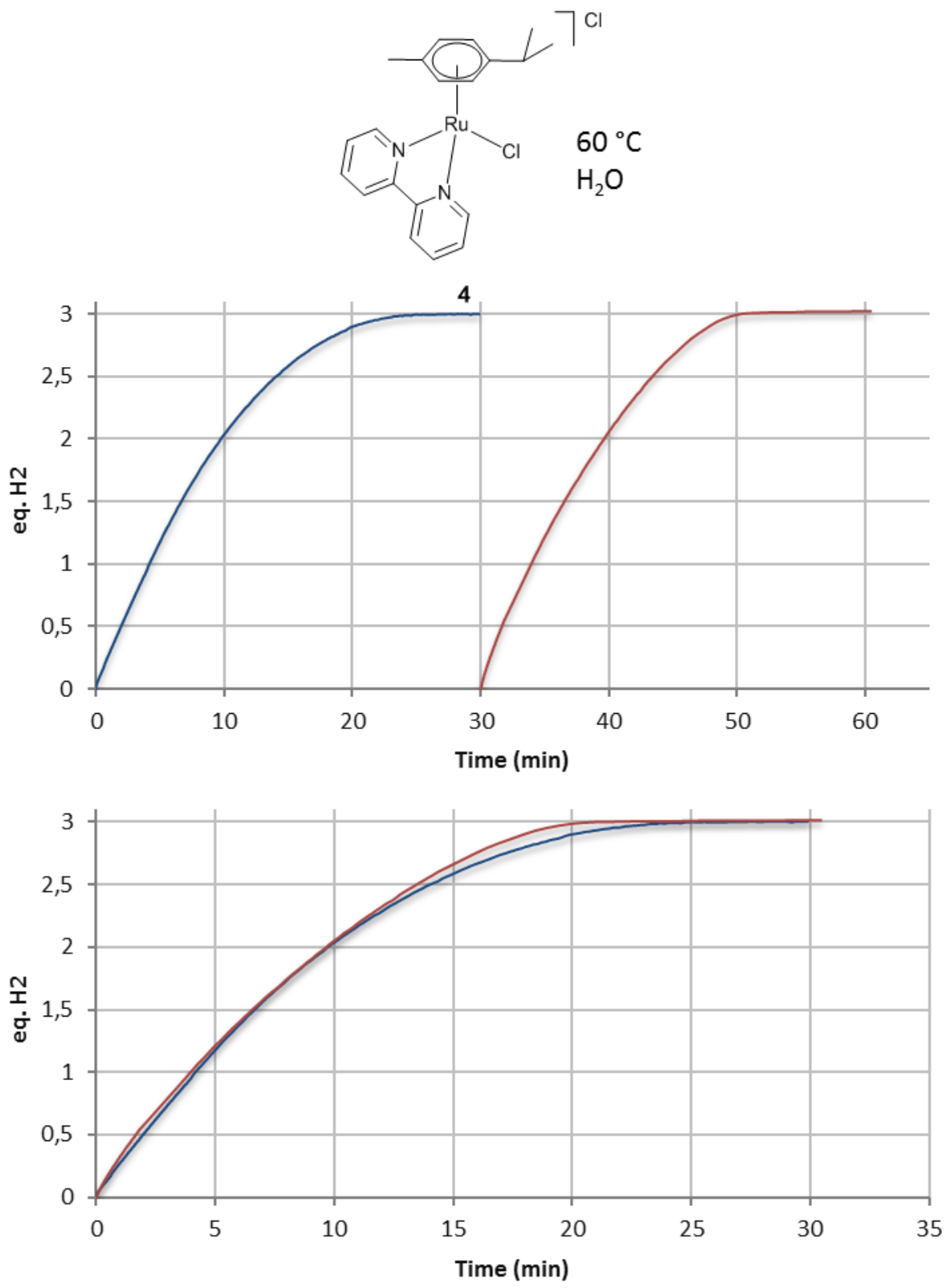

- 1st addition - 2nd addition

Figure S12. Reaction profiles of successive $A B$ hydrolysis using $[R u(p-C y m)($ bipy $) \mathrm{Cl}] \mathrm{Cl}(\mathbf{V})$ as precatalyst. (Table 1 entry 7). Conditions: $[\mathrm{Ru}]=2.3 \mathrm{mM},[\mathrm{AB}]=0.46 \mathrm{M}, 1.5 \mathrm{~mL} \mathrm{H} \mathrm{O}_{2}, 6{ }^{\circ} \mathrm{C}$. BOTTOM: Superimposed reaction profiles of successive reactions. 


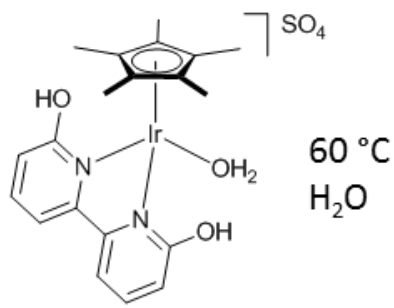

3
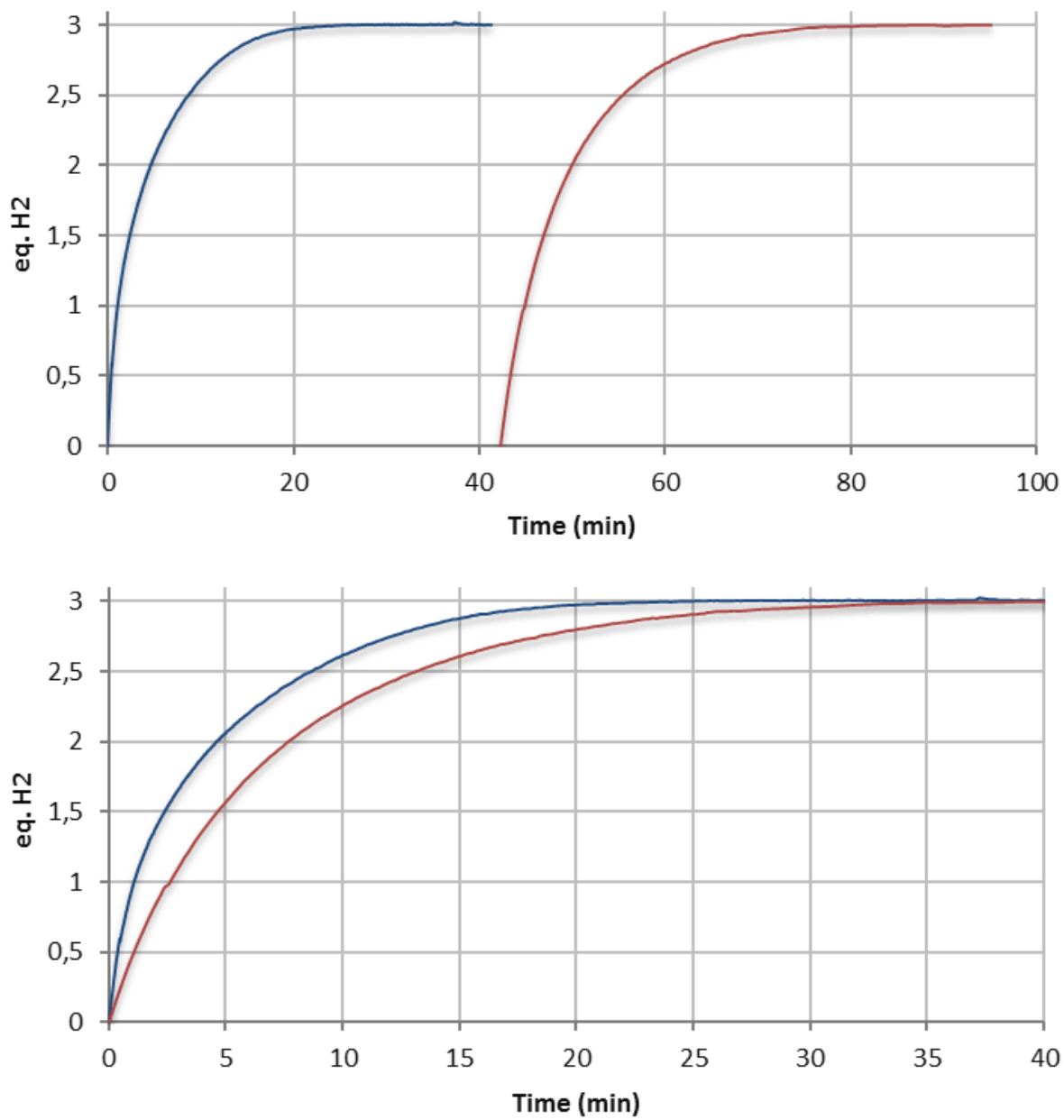

-1st addition -2 nd addition

Figure S13. Reaction profiles of successive $A B$ hydrolysis using [IrCp*(bipy-OH) $\left.\left(\mathrm{H}_{2} \mathrm{O}\right)\right] \mathrm{SO}_{4}$ (Himeda's catalyst) (VI). (Table 1, Entry 8). Conditions: $[\mathrm{Ir}]=2.3 \mathrm{mM},[\mathrm{AB}]=0.46 \mathrm{M}, 1.5 \mathrm{~mL} \mathrm{H} \mathrm{H}_{2} \mathrm{O}$, $60^{\circ} \mathrm{C}$. Additional $21.3 \mathrm{mg}$ of $\mathrm{AB}$ for the consecutive reaction. BOTTOM: Superimposed reaction profiles of successive reactions. 
$[\mathrm{Ru}(\mathrm{pCym})(\mathrm{dmbp}) \mathrm{Cl}] \mathrm{Cl},\left(\mathrm{dmbp}=5,5^{\prime}\right.$-dimethoxybipyridine) intended to be a non protonresponsive analog of compound $\mathbf{1}$ was originally synthesized and studied, for comparative purposes. The sigmoidal reaction profiles obtained, (Figures S14 and S15) together with $\mathrm{Hg}$ poisoning test (Figure S16) suggested that, in this case, the catalytic activity could be partially attributed to decomposition to Ru-NPs. Further studies on the compound stability by NMR spectroscopy confirmed that it is a rather unstable compound in solution. Therefore, it has not been included in the main manuscript, but it is included here to help other researchers in further studies of related systems.
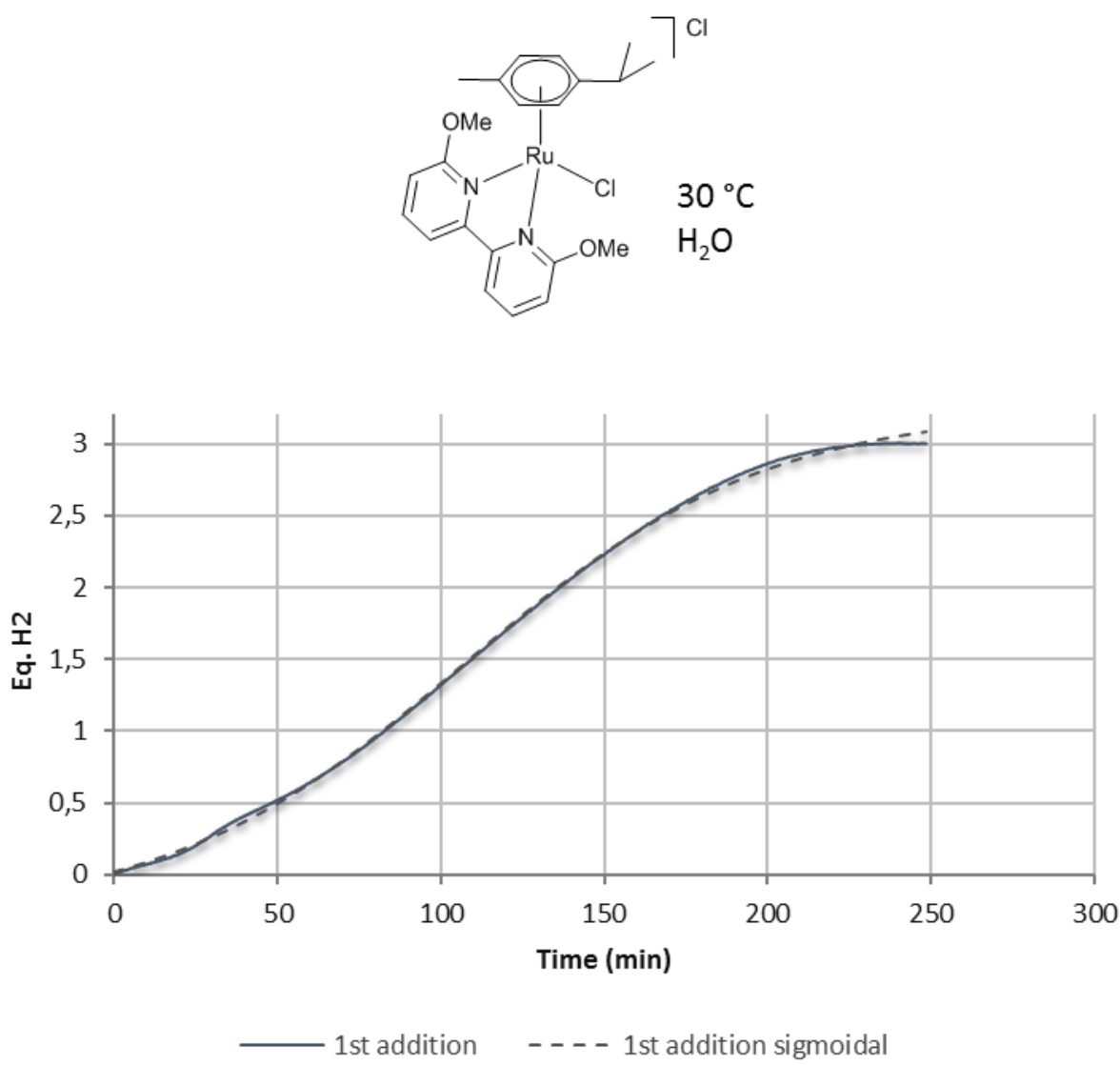

Figure S14. Reaction profile of $A B$ hydrolysis using $[R u(p-C y m)(d m o b p) C l] C l\left(d m o b p=6,6^{\prime}\right.$ dimethoxybipyridine) as precatalyst. Sigmoidal fitting shown. (not discussed in manusctipt). Conditions: $[\mathrm{Ru}]=2.3 \mathrm{mM},[\mathrm{AB}]=0.46 \mathrm{M}, 1.5 \mathrm{~mL} \mathrm{H} \mathrm{H}_{2} \mathrm{O}, 30^{\circ} \mathrm{C}$. 

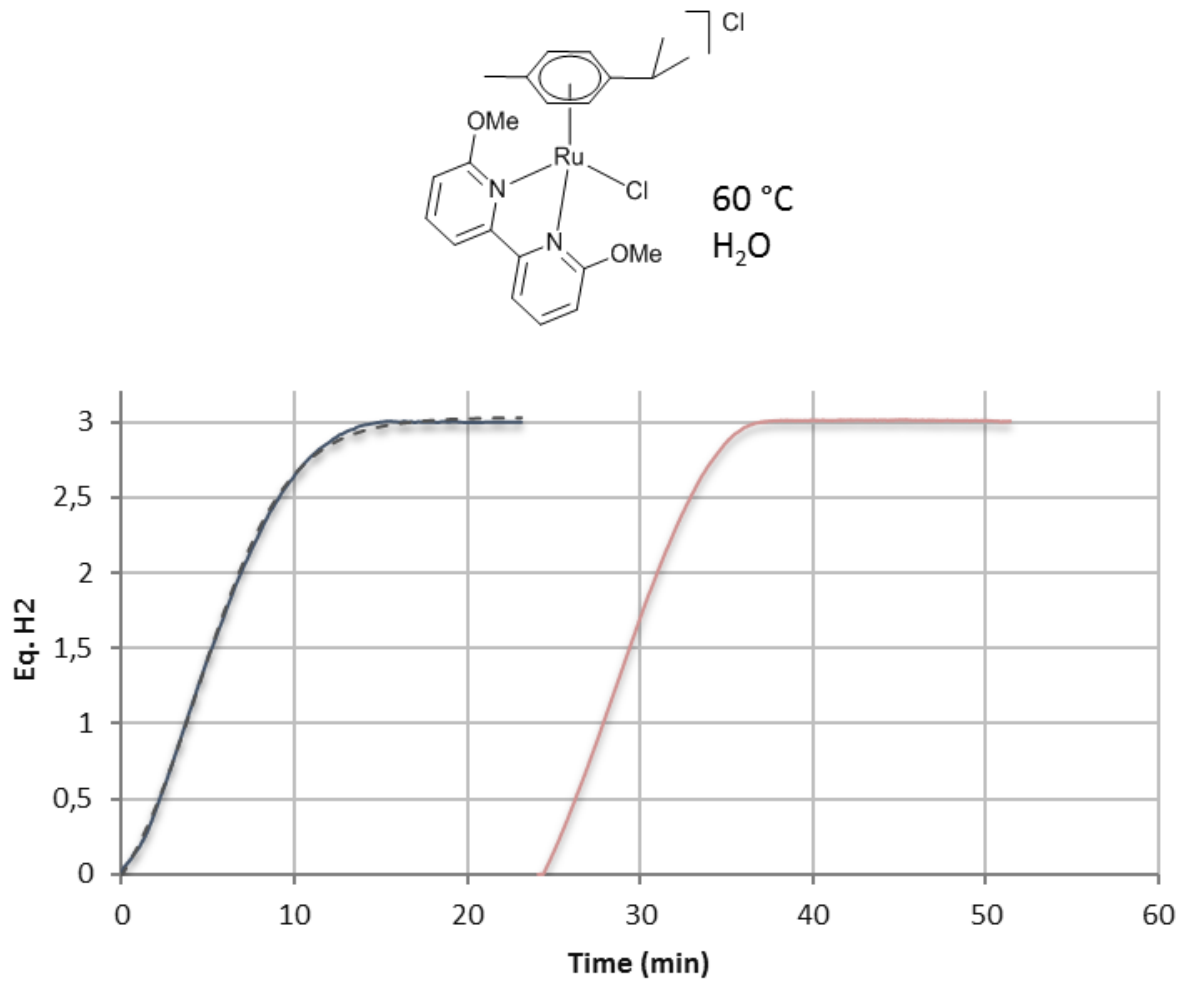

— 1st addition - - - - 1st addition sigmoidal _ $\quad$ 2nd addition sigmoidal

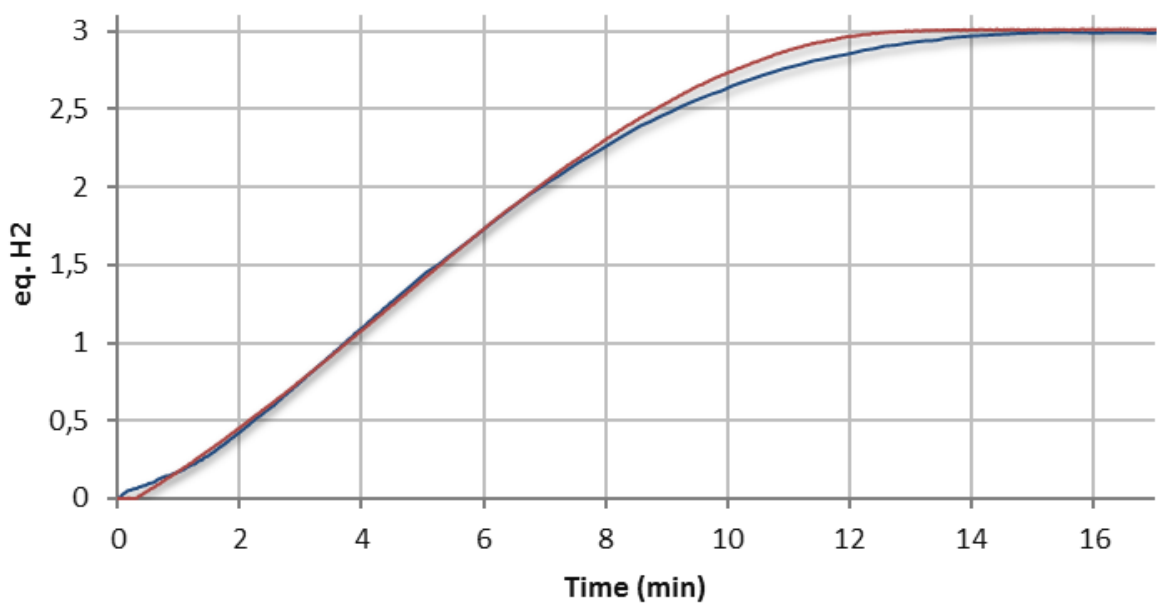

- 1st addition $\quad$-2nd addition

Figure S15. Reaction profiles of successive $A B$ hydrolysis using $[R u(p-C y m)(d m o b p) C l] C l$ as precatalyst. (not discussed in manuscript). Conditions: $[\mathrm{Ru}]=2.3 \mathrm{mM},[\mathrm{AB}]=0.46 \mathrm{M}, 1.5 \mathrm{~mL} \mathrm{H}_{2} \mathrm{O}$, $60{ }^{\circ} \mathrm{C}$. Additional $21.3 \mathrm{mg}$ of $A B$ for the consecutive reaction. TOP: Consecutive profiles. Sigmoidal fitting shown for the first addition. BOTTOM: Superimposed profiles (no induction period observed in the second addition). 

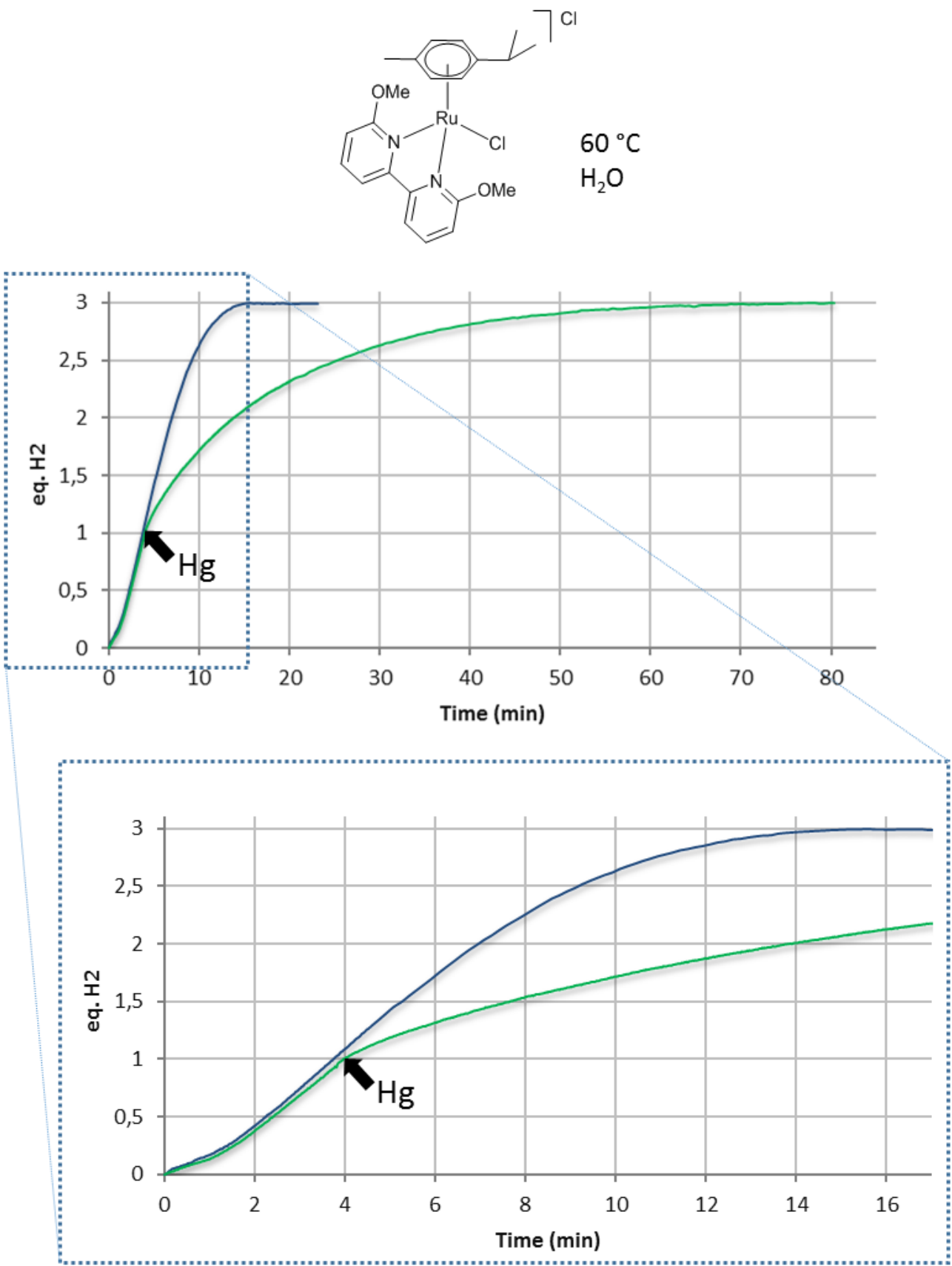

—1st addition — addition of $\mathrm{Hg}$

Figure S16. Reaction profiles of $A B$ hydrolysis using $[\mathrm{Ru}(\mathrm{p}-\mathrm{Cym})(\mathrm{dmobp}) \mathrm{Cl}] \mathrm{Cl}$ as precatalyst. (not discussed in manuscript). Conditions: $[R u]=2.3 \mathrm{mM},[\mathrm{AB}]=0.46 \mathrm{M}, 1.5 \mathrm{~mL} \mathrm{H} \mathrm{O}_{2}, 60^{\circ} \mathrm{C}$. A reaction profile of a run in which $\mathrm{Hg}$ (1000 eq. per Ru) was added after liberation of 1 eq. of $\mathrm{H}_{2}$ has been superimposed. 

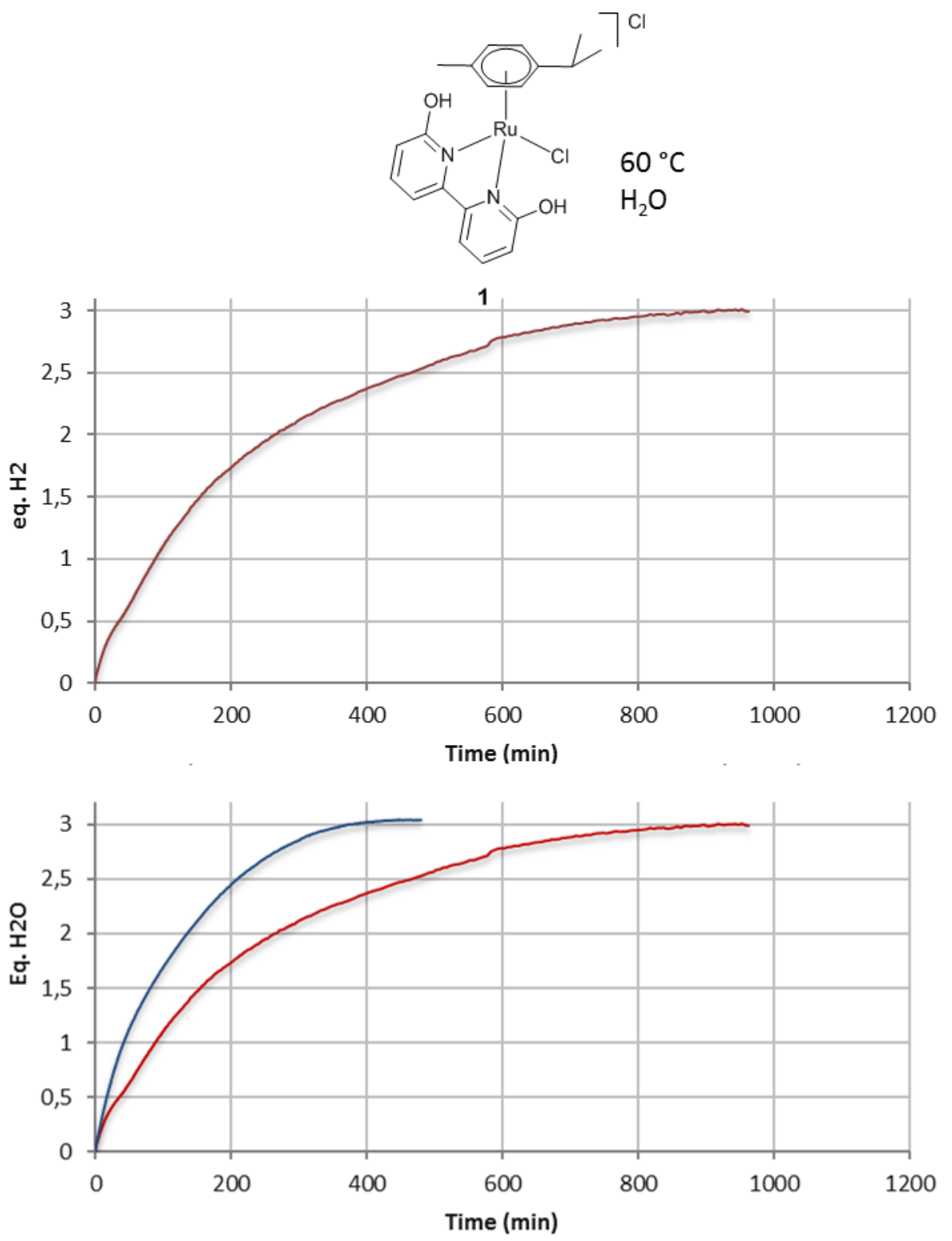

Second $A B$ addition on a reaction in $\mathrm{H}_{2} \mathrm{O}$

$\mathrm{NH}_{3} 0.46 \mathrm{M}$

Figure S17. Reaction profiles of top: $A B$ hydrolysis using $[\mathrm{Ru}(\mathrm{p}-\mathrm{Cym})(\mathrm{dhbp}) \mathrm{Cl}] \mathrm{Cl}(\mathbf{1})$ as precatalyst. (Table 2, Entry 1). Conditions: $[\mathrm{Ru}]=2.3 \mathrm{mM},[\mathrm{AB}]=0.46 \mathrm{M}, 1.5 \mathrm{~mL}$ of a $\mathrm{NH}_{3}$ solution $0.46 \mathrm{M}$ in $\mathrm{H}_{2} \mathrm{O}, 60^{\circ} \mathrm{C}$. BOTTOM: Comparison of the reaction profile after a second addition of $A B$ on a hydrolysis reaction using catalyst 1 in $\mathrm{H}_{2} \mathrm{O}\left([\mathrm{Ru}]=2.3 \mathrm{mM},[\mathrm{AB}]=0.46 \mathrm{M}, 1.5\right.$ of $\mathrm{H}_{2} \mathrm{O}, 60^{\circ} \mathrm{C}$, blue line), and the reaction profile in presence of $\mathrm{NH}_{3}$ (red line). 


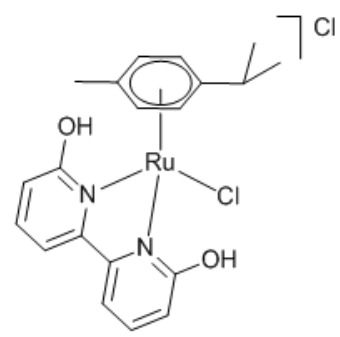

$60^{\circ} \mathrm{C}$

$\mathrm{H}_{2} \mathrm{O}, \mathrm{pH} 9.0$
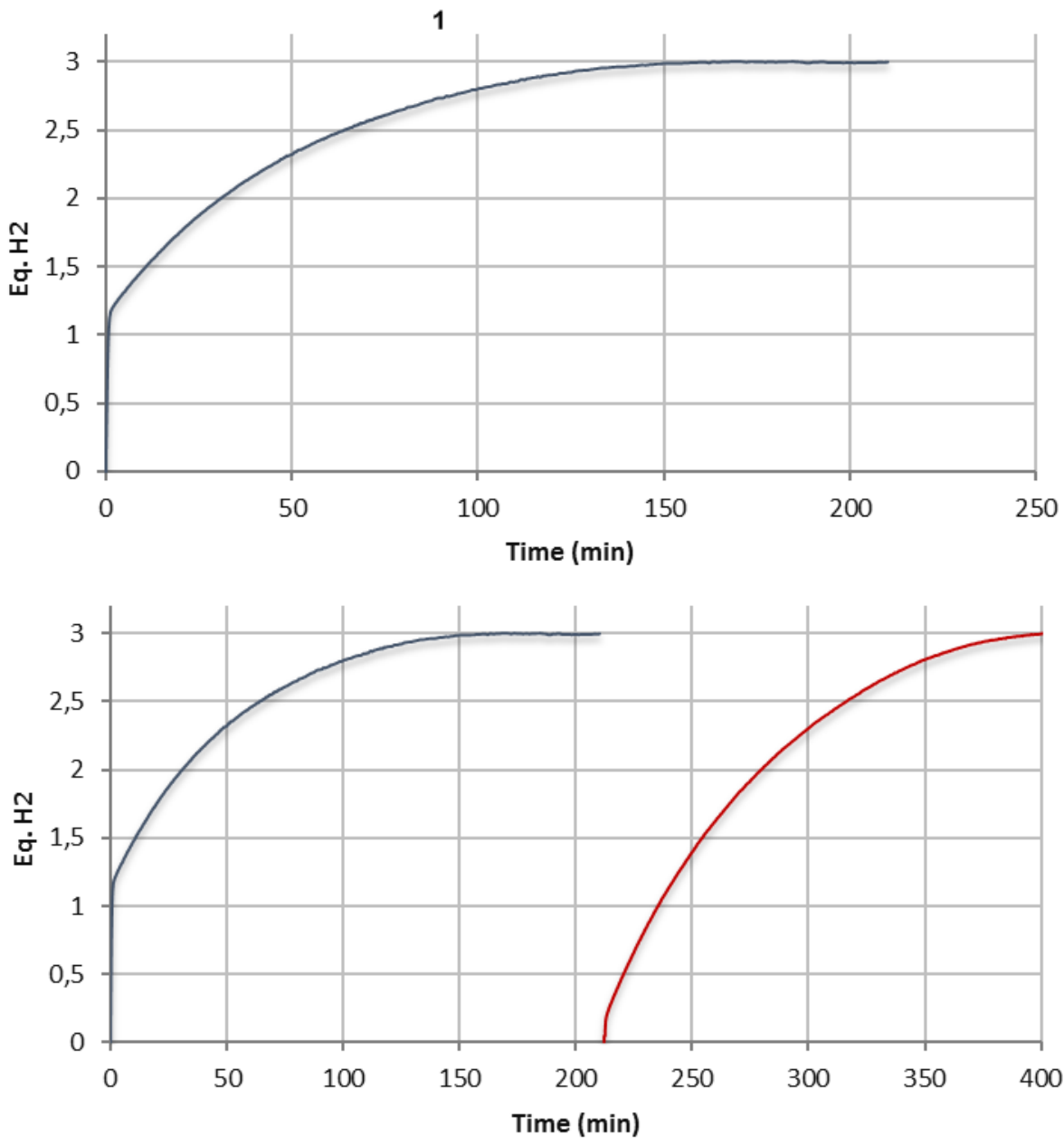

- 1 st addition $\quad$ 2nd addition

Figure S18. Reaction profiles of successive $A B$ solvolysis using $[\mathrm{Ru}(\mathrm{p}-\mathrm{Cym})(\mathrm{dhbp}) \mathrm{Cl}] \mathrm{Cl}(\mathbf{1})$. (Table 2, Entry 2). Conditions: $[\mathrm{Ru}]=2.3 \mathrm{mM},[\mathrm{AB}]=0.46 \mathrm{M}, 1.5 \mathrm{~mL} \mathrm{NaOH} / \mathrm{H}_{2} \mathrm{O}(\mathrm{pH}=9.0), 60{ }^{\circ} \mathrm{C}$. Additional $21.3 \mathrm{mg}$ of $A B$ for the consecutive reaction. BOTTOM: Reaction profiles of successive reactions. 

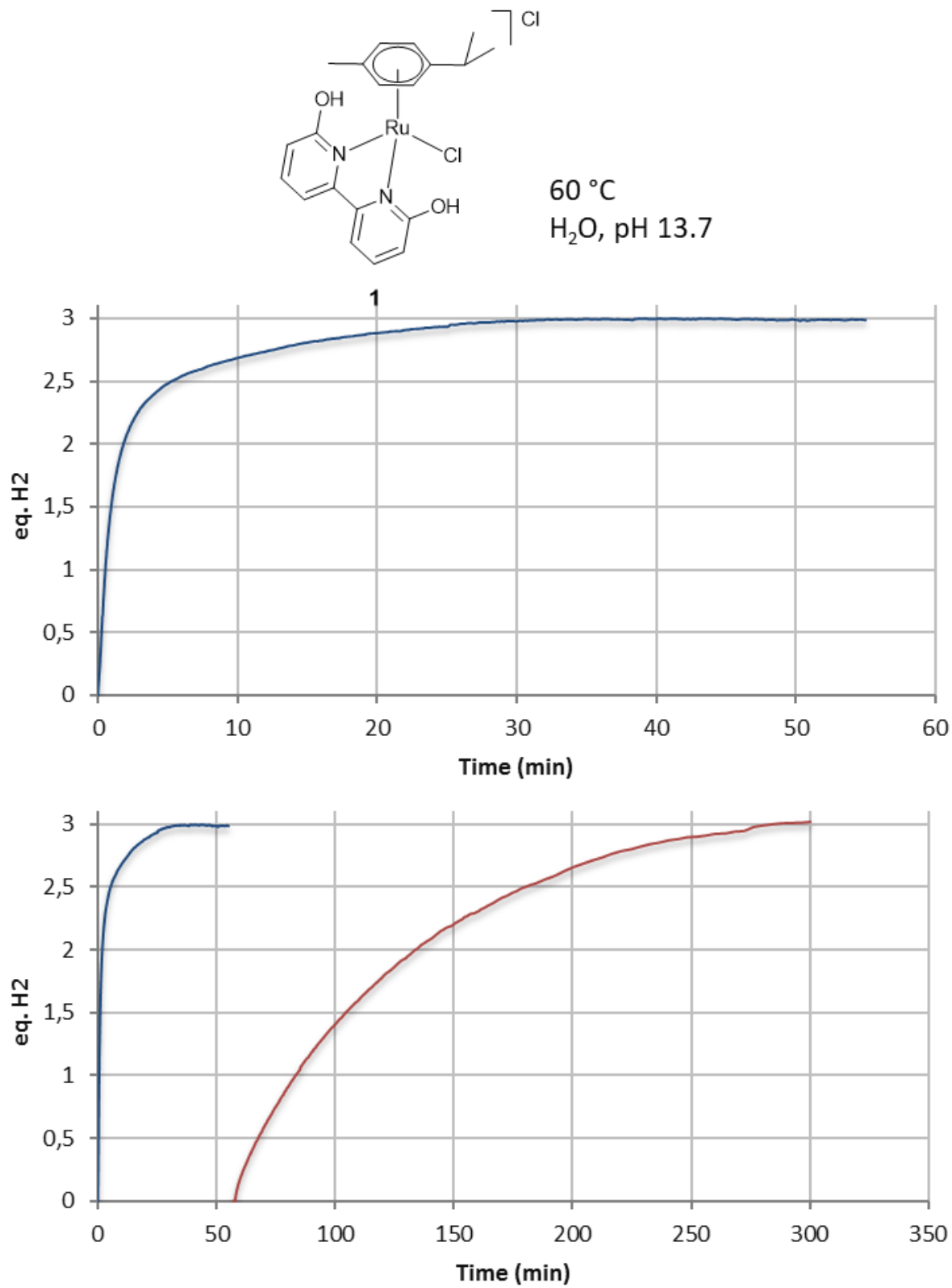

-1 st addition $\quad$ 2nd addition

Figure S19. Reaction profiles of successive $A B$ solvolysis using $[\mathrm{Ru}(\mathrm{p}-\mathrm{Cym})(\mathrm{dhbp}) \mathrm{Cl}] \mathrm{Cl}(\mathbf{1})$. (Table 2, Entry 3). Conditions: $[\mathrm{Ru}]=2.3 \mathrm{mM},[\mathrm{AB}]=0.46 \mathrm{M}, 1.5 \mathrm{~mL} \mathrm{NaOH} / \mathrm{H}_{2} \mathrm{O} 0.46 \mathrm{M}(\mathrm{pH}=13.7), 60$ ${ }^{\circ} \mathrm{C}$. Additional $21.3 \mathrm{mg}$ of $\mathrm{AB}$ for the consecutive reaction. BOTTOM: Reaction profiles of successive reactions. 


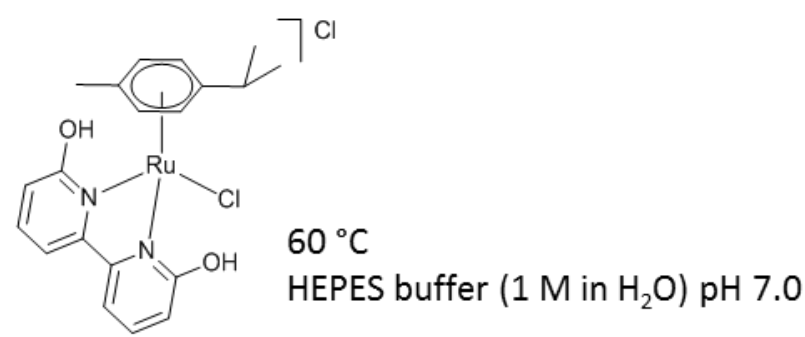

1
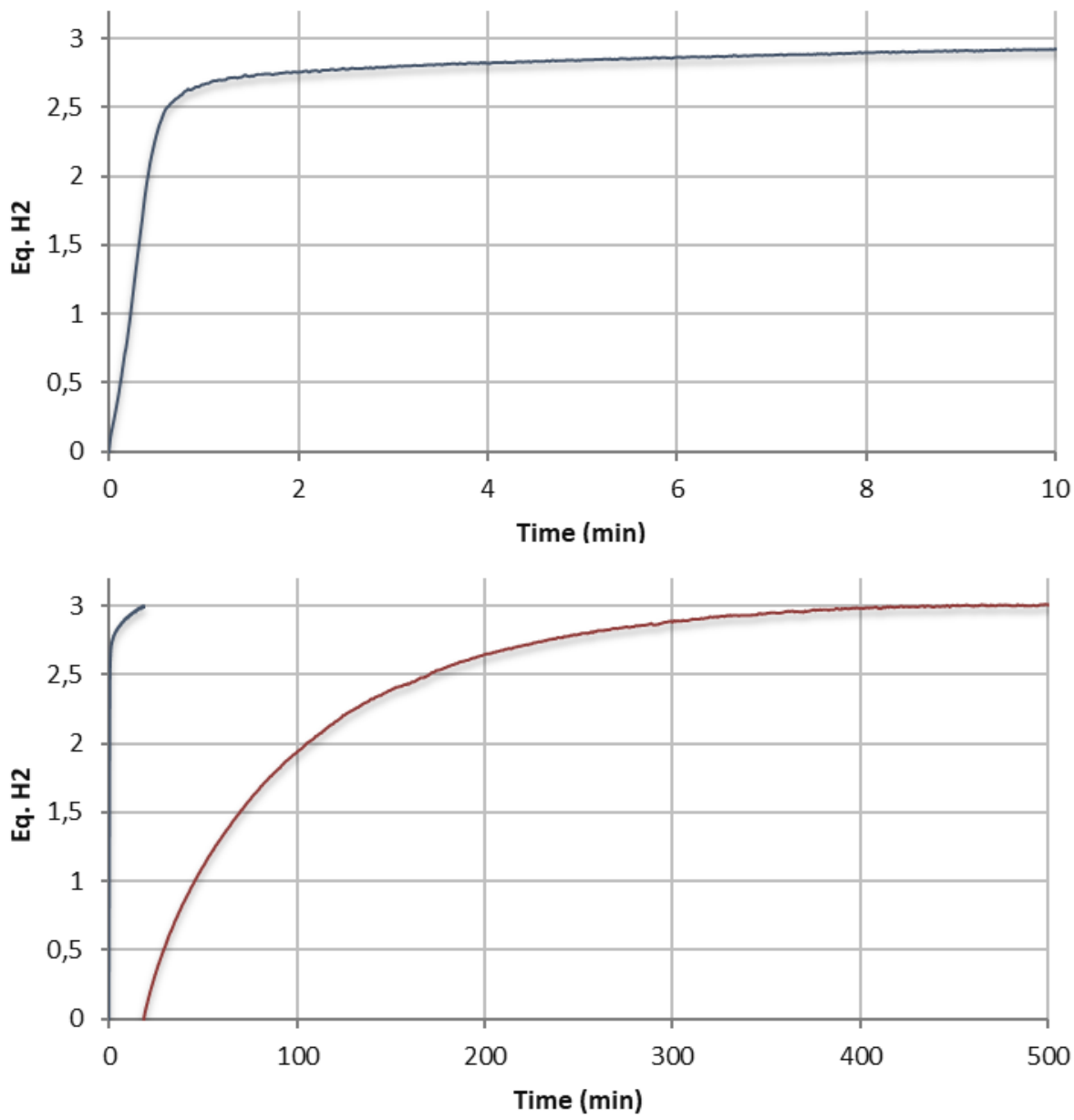

- 1st addition $\quad$ 2nd addition

Figure S20. Reaction profiles of successive $A B$ solvolysis using $[R u(p-C y m)(d h b p) C l] C l(1)$. (Table 2, Entry 4). Conditions: $[R u]=2.3 \mathrm{mM},[A B]=0.46 \mathrm{M}, 1.5 \mathrm{~mL}$ (HEPES buffer $1 \mathrm{M}$ in $\mathrm{H}_{2} \mathrm{O}, \mathrm{pH} 7.0$ ), $60{ }^{\circ} \mathrm{C}$. Additional $21.3 \mathrm{mg}$ of $\mathrm{AB}$ for the consecutive reaction. BOTTOM: Reaction profiles of successive reactions. 

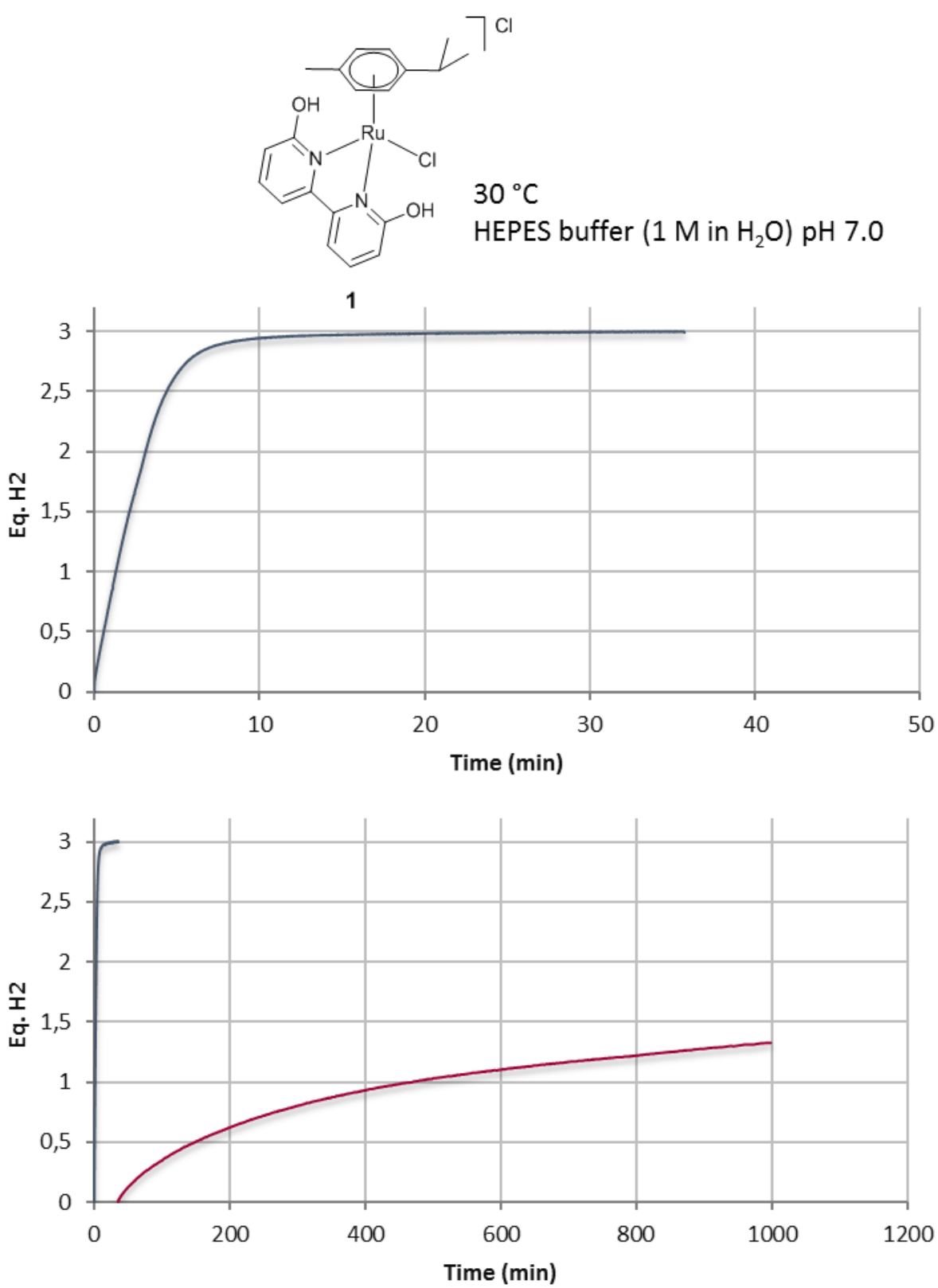

- 1 st addition $\quad$ 2nd addition

Figure S21. Reaction profiles of successive $A B$ solvolysis using $[\mathrm{Ru}(\mathrm{p}-\mathrm{Cym})(\mathrm{dhbp}) \mathrm{Cl}] \mathrm{Cl}(\mathbf{1})$. (Table 2, Entry 5). Conditions: $[R u]=2.3 \mathrm{mM},[A B]=0.46 \mathrm{M}, 1.5 \mathrm{~mL}$ (HEPES buffer $1 \mathrm{M}$ in $\mathrm{H}_{2} \mathrm{O}, \mathrm{pH} 7.0$ ), $30{ }^{\circ} \mathrm{C}$. Additional $21.3 \mathrm{mg}$ of $\mathrm{AB}$ for the consecutive reaction. BOTTOM: Reaction profiles of successive reactions. 


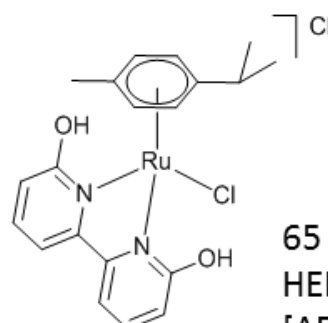

$65^{\circ} \mathrm{C}$

HEPES buffer $\left(1 \mathrm{M}\right.$ in $\left.\mathrm{H}_{2} \mathrm{O}\right) \mathrm{pH} 7.0$

$[\mathrm{AB}] /[\mathrm{cat}]=1000$
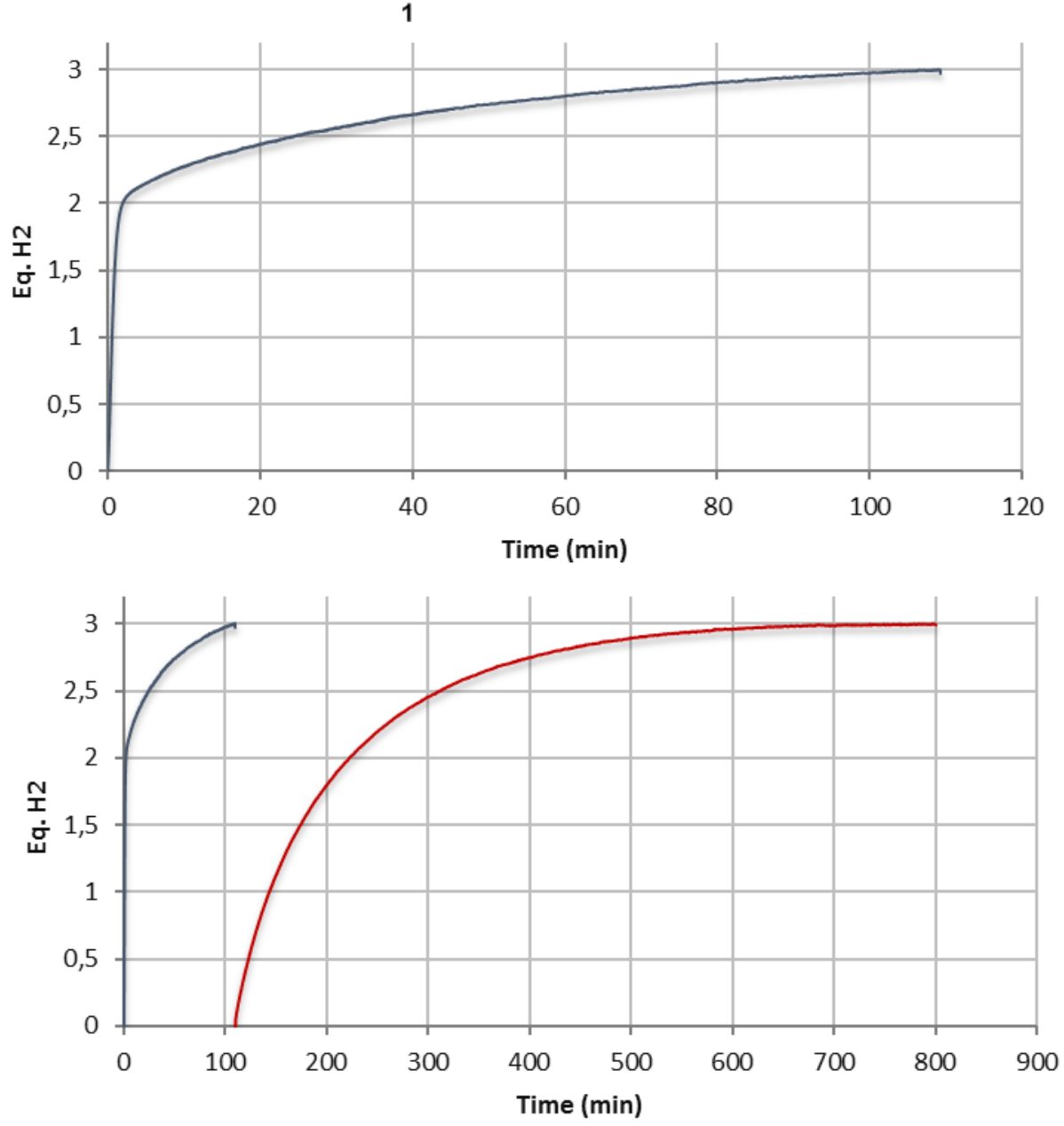

$\longrightarrow$ 1st addition $\quad$ 2nd addition

Figure S22. Reaction profiles of successive $A B$ solvolysis using $[\mathrm{Ru}(\mathrm{p}-\mathrm{Cym})(\mathrm{dhbp}) \mathrm{Cl}] \mathrm{Cl}(\mathbf{1})$. (Table 2, Entry 6). Conditions: $[R u]=0.1 \mathrm{mM},[A B]=0.1 \mathrm{M}, 5 \mathrm{~mL}$ (HEPES buffer $1 \mathrm{M}$ in $\mathrm{H}_{2} \mathrm{O}, \mathrm{pH} 7.0$ ), 65 ${ }^{\circ} \mathrm{C}$. Additional $15.4 \mathrm{mg}$ of $\mathrm{AB}$ for the consecutive reaction. BOTTOM: Reaction profiles of successive reactions. 

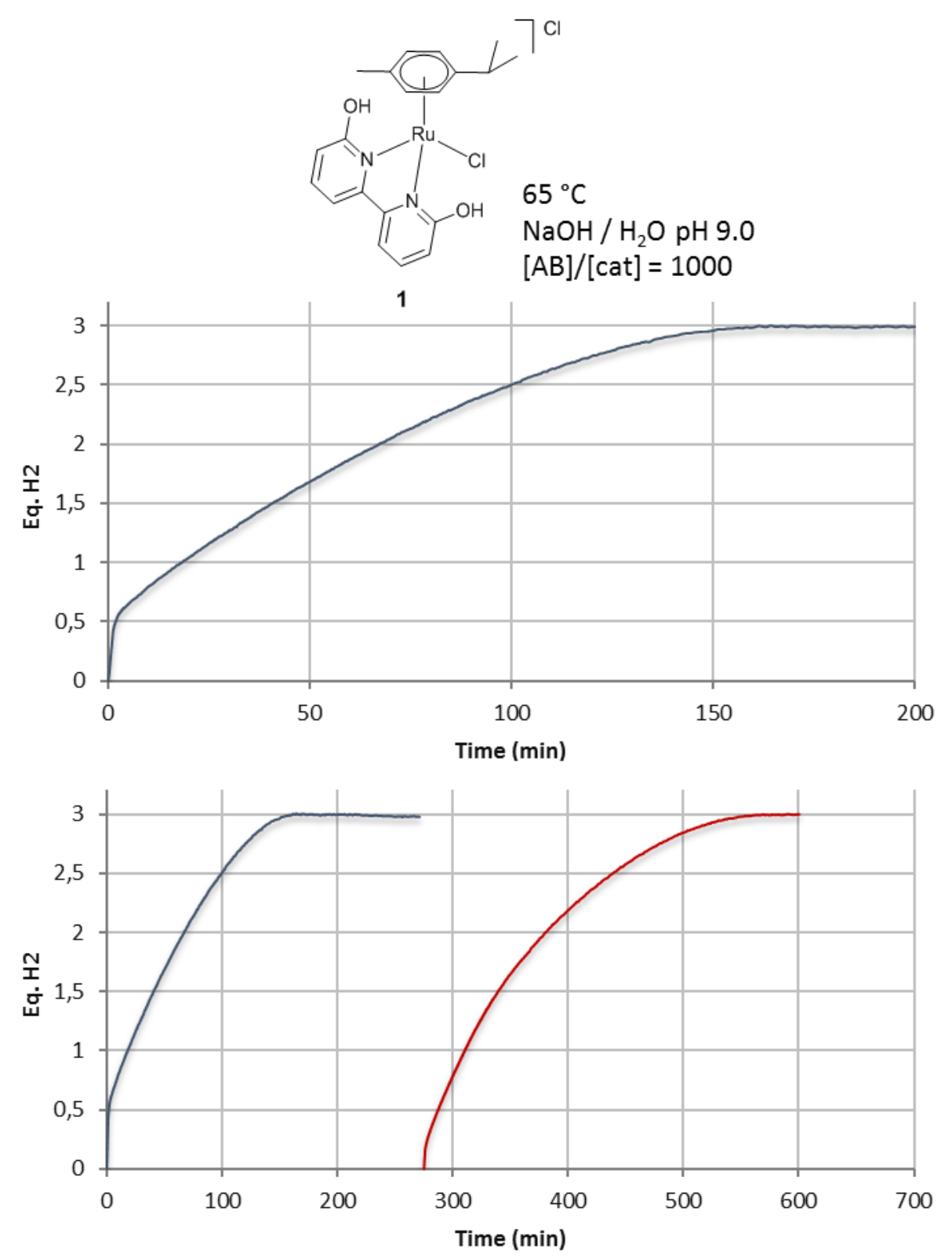

-1st addition $\quad$ 2nd addition

Figure S23. Reaction profiles of successive $A B$ solvolysis using $[\mathrm{Ru}(\mathrm{p}-\mathrm{Cym})(\mathrm{dhbp}) \mathrm{Cl}] \mathrm{Cl}(\mathbf{1})$. (Table 2, Entry 7). Conditions: $[\mathrm{Ru}]=0.1 \mathrm{mM},[\mathrm{AB}]=0.1 \mathrm{M}, 5 \mathrm{~mL}\left(\mathrm{H}_{2} \mathrm{O}, \mathrm{pH} 9.0\right), 65^{\circ} \mathrm{C}$. Additional 15.4 $\mathrm{mg}$ of $A B$ for the consecutive reaction. BOTTOM: Reaction profiles of successive reactions. 


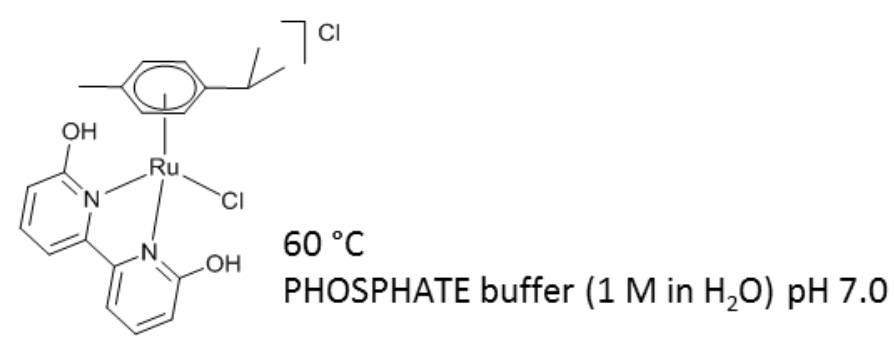

1

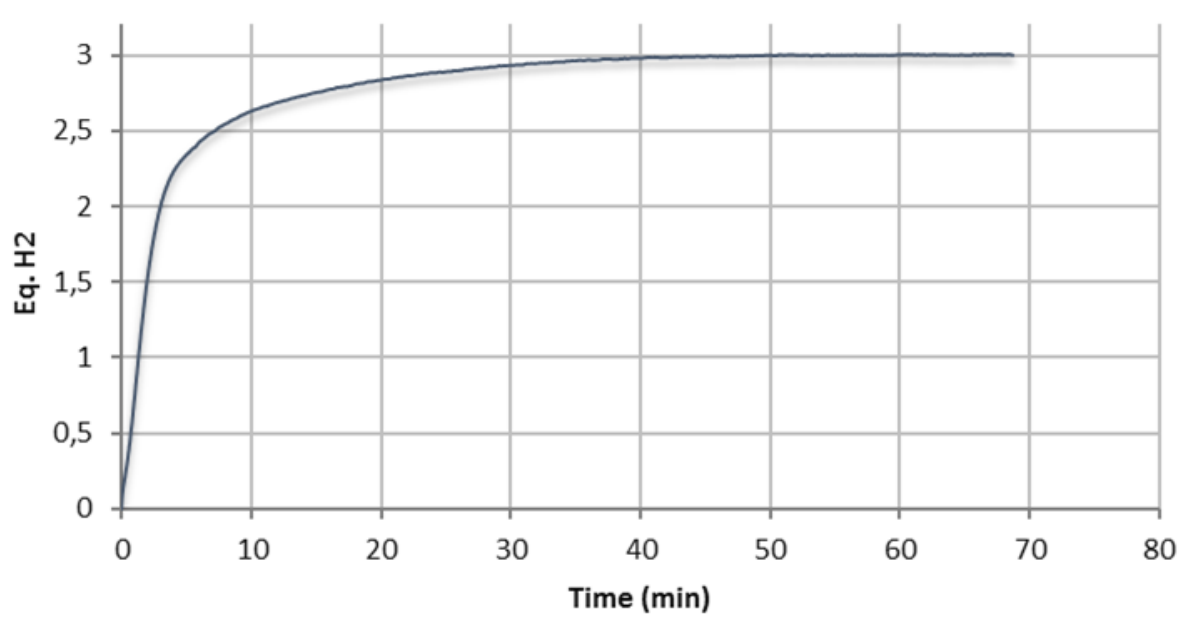

Figure S24. Reaction profile of $A B$ solvolysis using $[R u(p-C y m)(d h b p) C l] C l(1)$. Conditions: $[R u]=$ $2.3 \mathrm{mM},[\mathrm{AB}]=0.46 \mathrm{M}, 1.5 \mathrm{~mL}$ (phosphate buffer $1 \mathrm{M}$ in $\mathrm{H}_{2} \mathrm{O}, \mathrm{pH} 7.0$ ), $60^{\circ} \mathrm{C}$. 

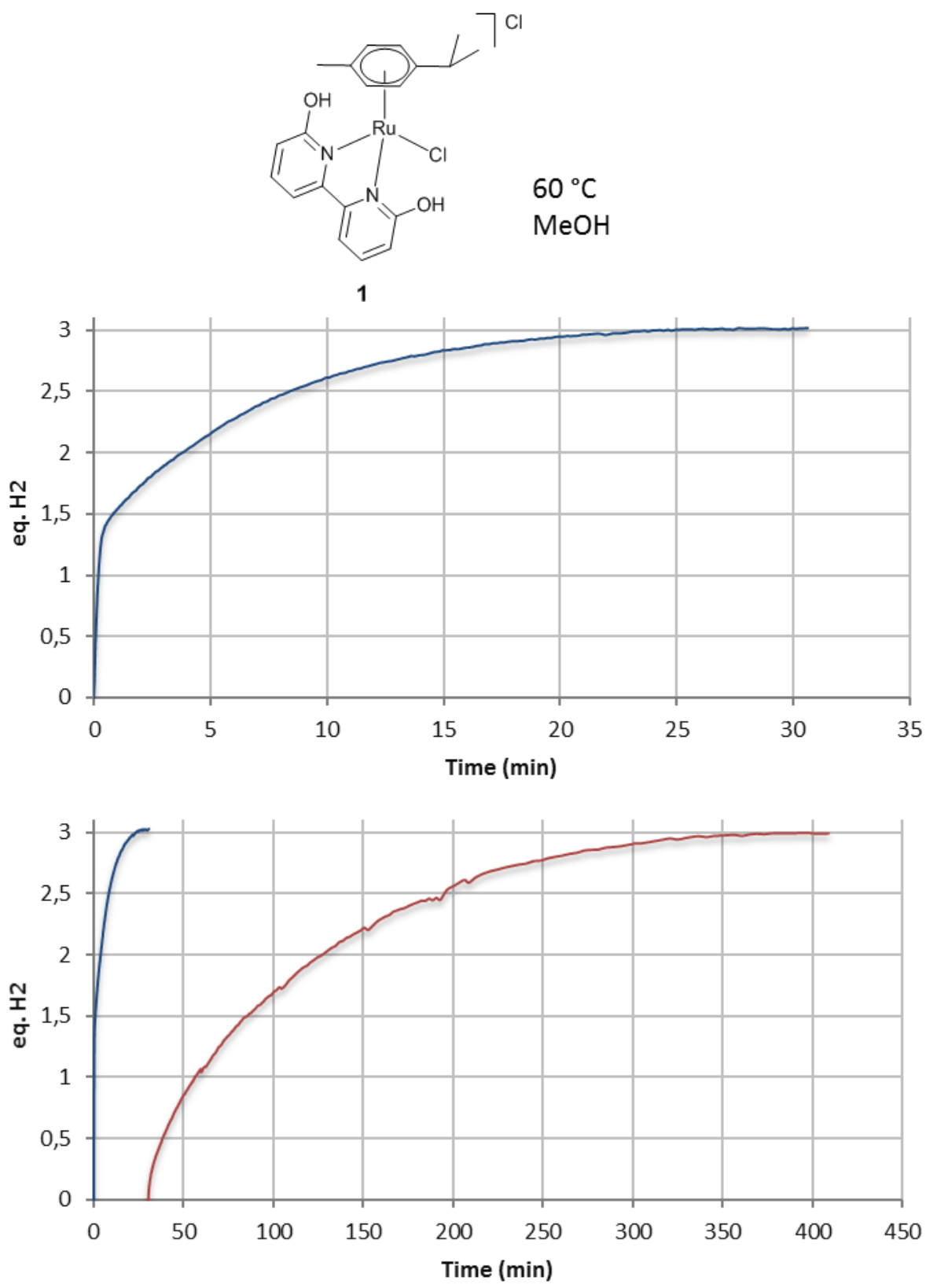

- 1 st addition $\quad$ 2nd addition

Figure S25. Reaction profiles of: TOP: $A B$ alcoholysis using $[\mathrm{Ru}(\mathrm{p}-\mathrm{Cym})(\mathrm{dhbp}) \mathrm{Cl}] \mathrm{Cl}(\mathbf{1})$. (Table 2, Entry 8). Conditions: $[\mathrm{Ru}]=2.3 \mathrm{mM},[\mathrm{AB}]=0.46 \mathrm{M}, 1.5 \mathrm{~mL} \mathrm{MeOH}, 60^{\circ} \mathrm{C}$. Additional $21.3 \mathrm{mg}$ of $A B$ for the consecutive reaction. BOTTOM: Reaction profiles of successive reactions. 

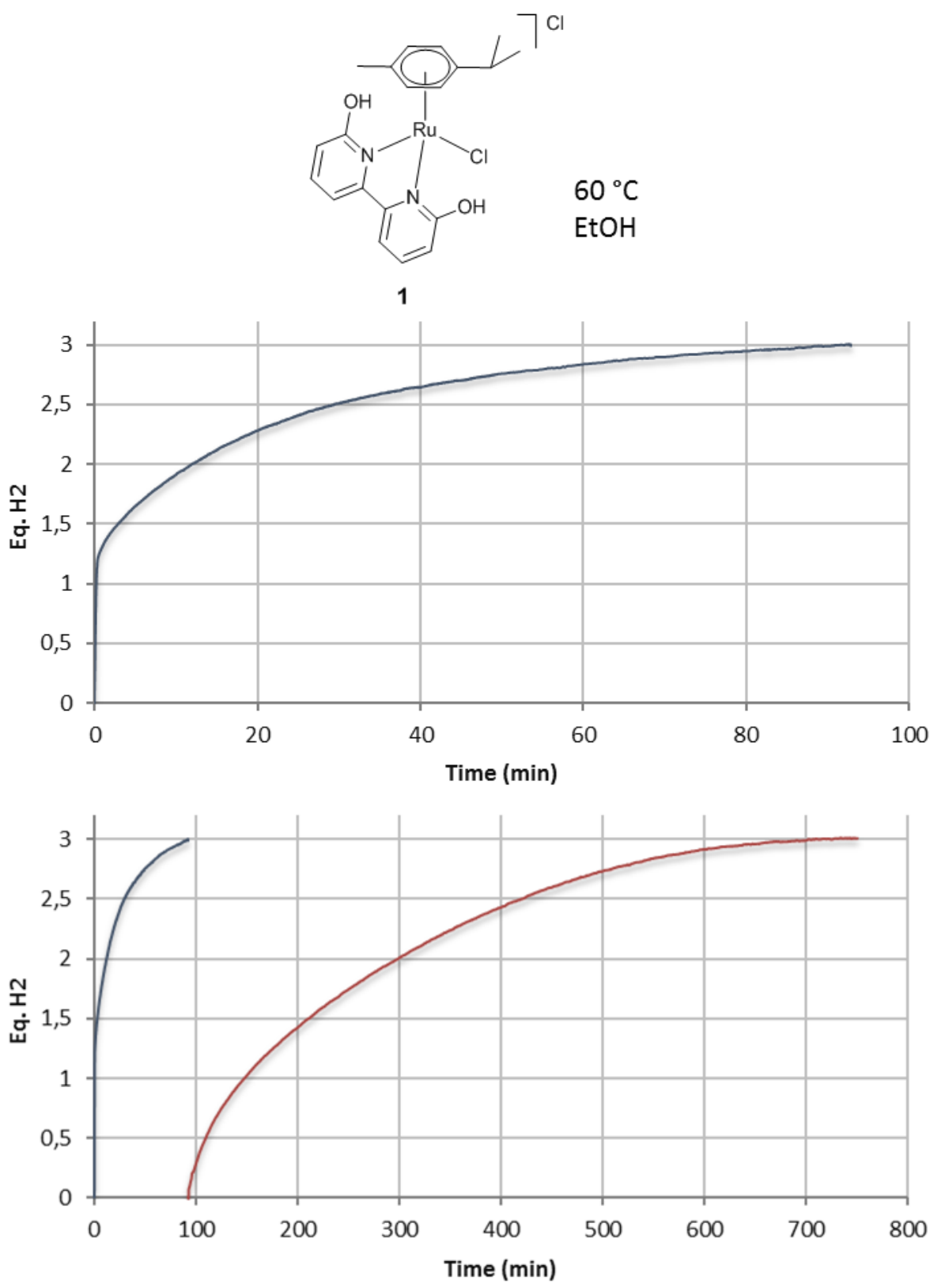

- 1 st addition $\quad$ 2nd addition

Figure S26. Reaction profiles of successive $A B$ alcoholysis using $[\mathrm{Ru}(\mathrm{p}-\mathrm{Cym})(\mathrm{dhbp}) \mathrm{Cl}] \mathrm{Cl}(\mathbf{1})$. (Table 2, Entry 9). Conditions: $[\mathrm{Ru}]=2.3 \mathrm{mM},[\mathrm{AB}]=0.46 \mathrm{M}, 1.5 \mathrm{~mL} \mathrm{EtOH}, 60^{\circ} \mathrm{C}$. Additional $21.3 \mathrm{mg}$ of $A B$ for the consecutive reaction. BOTTOM: Reaction profiles of successive reactions. 


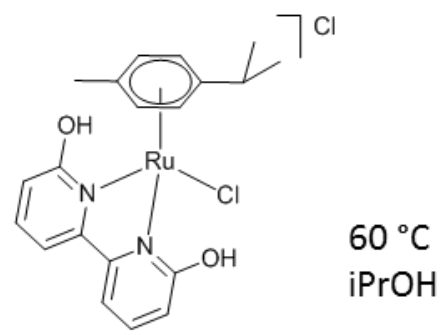

1
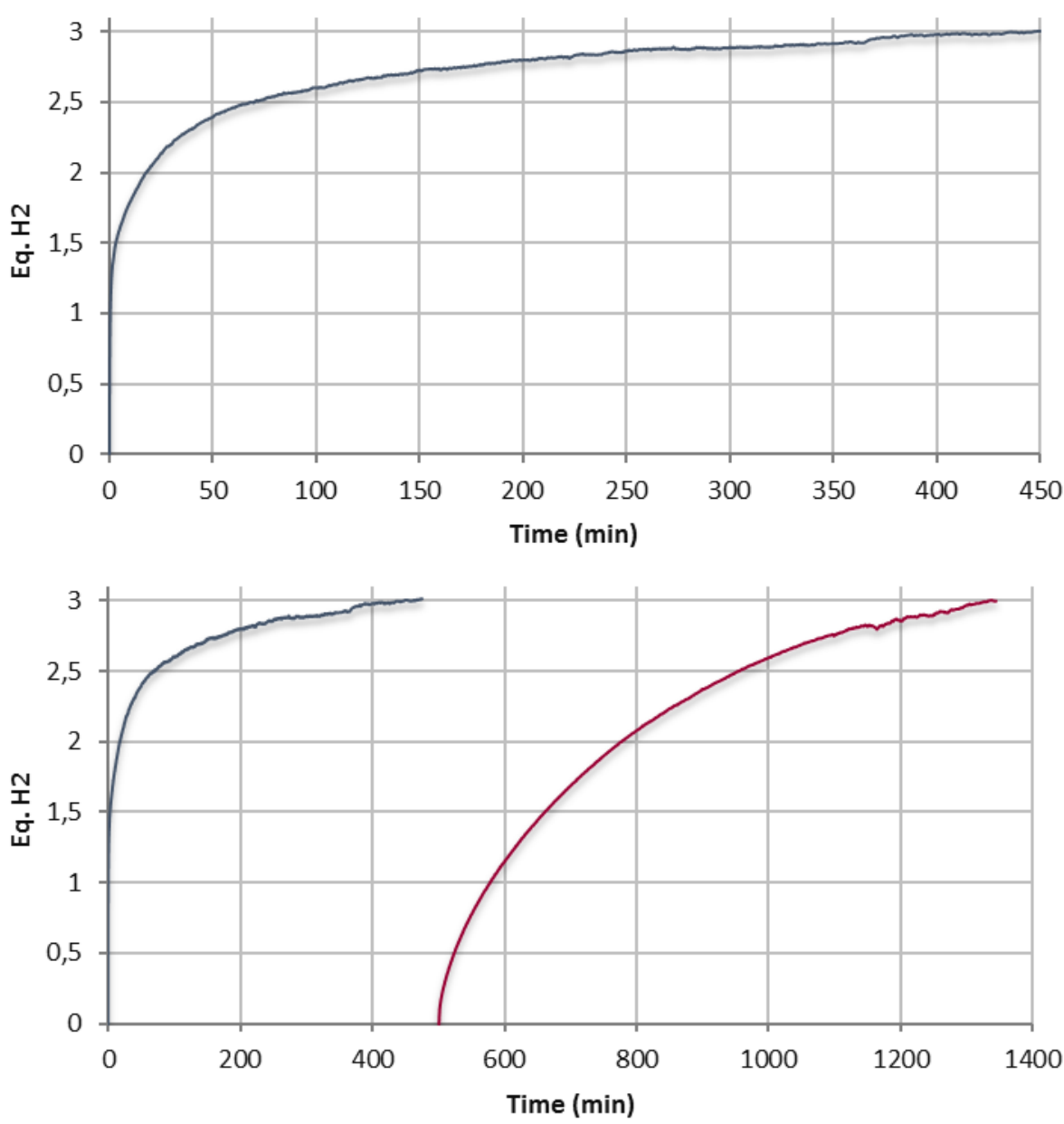

- 1 st addition $\quad$ 2nd addition

Figure S27. Reaction profiles of successive $A B$ alcoholysis using $[\mathrm{Ru}(\mathrm{p}-\mathrm{Cym})(\mathrm{dhbp}) \mathrm{Cl}] \mathrm{Cl}(\mathbf{1})$. (Table 2, Entry 10). Conditions: $[\mathrm{Ru}]=2.3 \mathrm{mM},[\mathrm{AB}]=0.46 \mathrm{M}, 1.5 \mathrm{~mL}$ iPrOH, $60^{\circ} \mathrm{C}$. Additional $21.3 \mathrm{mg}$ of $A B$ for the consecutive reaction. BOTTOM: Reaction profiles of successive reactions. 


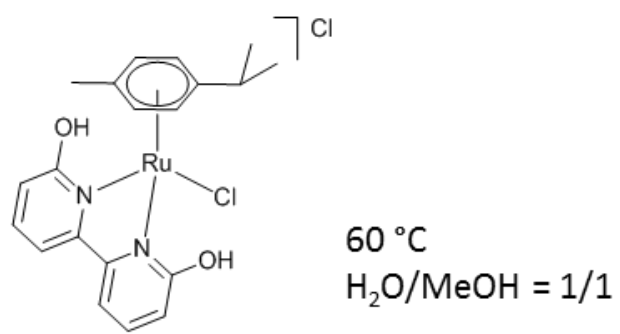

1
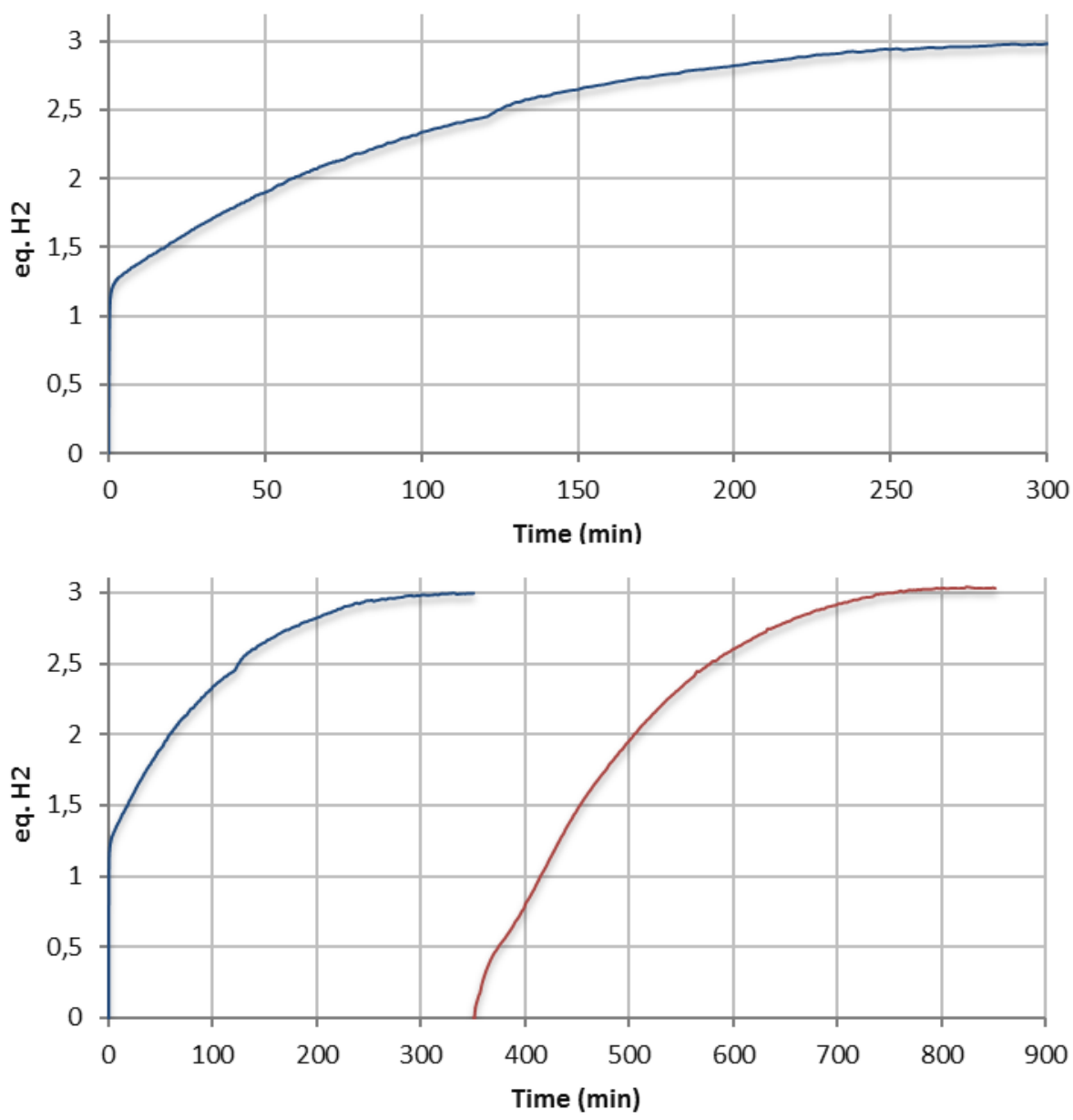

-1st addition $\quad$ 2nd addition

Figure S28. Reaction profiles of successive $A B$ solvolysis using $[\mathrm{Ru}(\mathrm{p}-\mathrm{Cym})(\mathrm{dhbp}) \mathrm{Cl}] \mathrm{Cl}(\mathbf{1})$. (Table 2, Entry 11). Conditions: $[\mathrm{Ru}]=2.3 \mathrm{mM},[\mathrm{AB}]=0.46 \mathrm{M}, 1.5 \mathrm{~mL} \mathrm{H} \mathrm{O}_{2} \mathrm{MeOH}=1 / 1,60^{\circ} \mathrm{C}$. Additional $21.3 \mathrm{mg}$ of $A B$ for the consecutive reaction. Sequential and superimposed reaction profiles shown. 


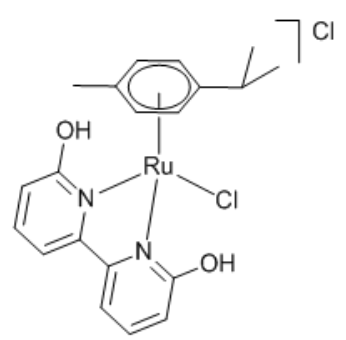

$60^{\circ} \mathrm{C}$

$\mathrm{H}_{2} \mathrm{O} / \mathrm{MeOH}=1 / 9$

1
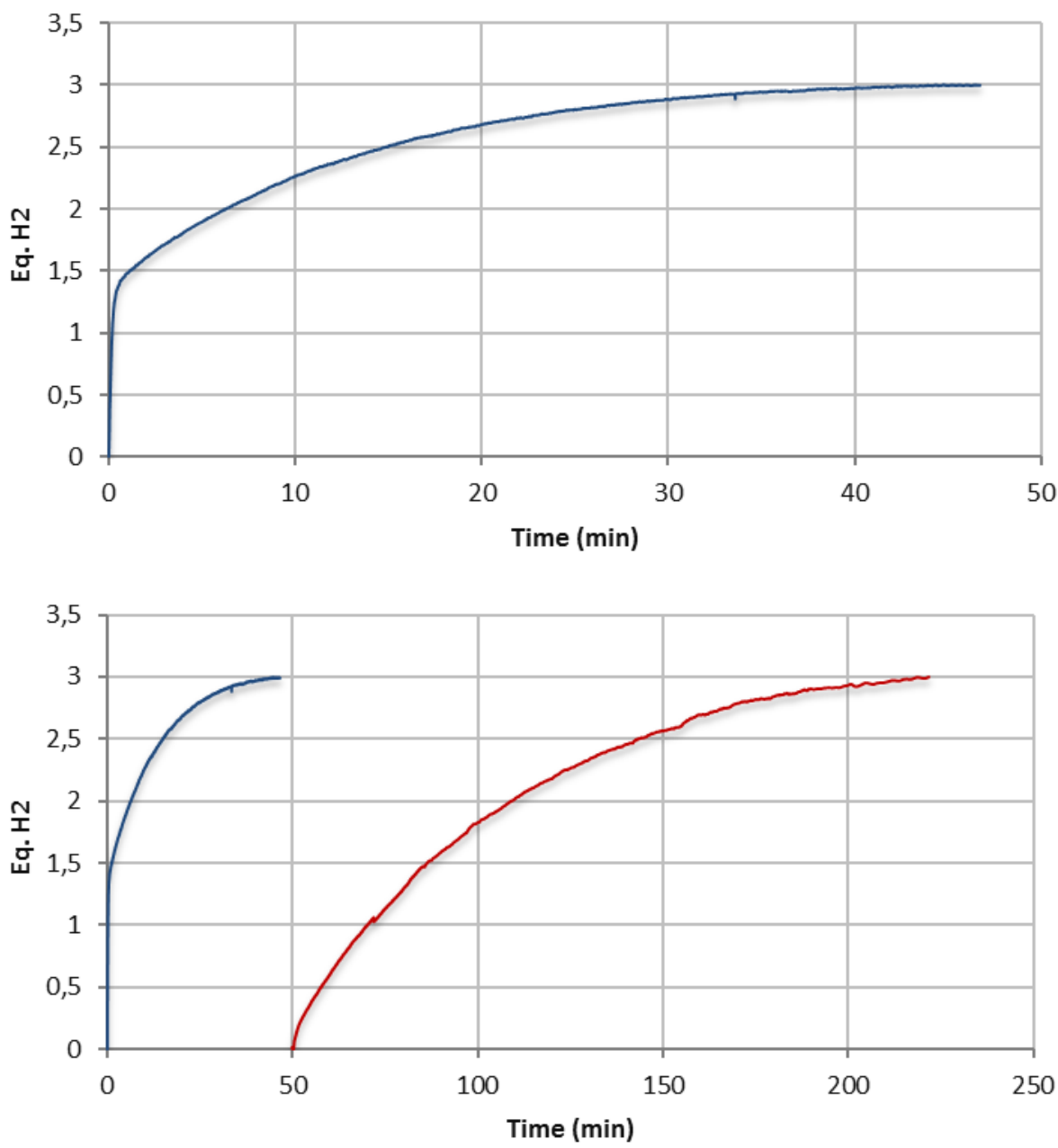

- 1 st addition $\quad$ 2nd addition

Figure S29. Reaction profiles of successive $A B$ solvolysis using $[\mathrm{Ru}(\mathrm{p}-\mathrm{Cym})(\mathrm{dhbp}) \mathrm{Cl}] \mathrm{Cl}(\mathbf{1})$. (Table 2, Entry 12). Conditions: $[\mathrm{Ru}]=2.3 \mathrm{mM},[\mathrm{AB}]=0.46 \mathrm{M}, 1.5 \mathrm{~mL} \mathrm{H} \mathrm{O}_{2} \mathrm{MeOH}=1 / 9,60^{\circ} \mathrm{C}$. Additional $21.3 \mathrm{mg}$ of $A B$ for the consecutive reaction. BOTTOM: Reaction profiles of successive reactions. 

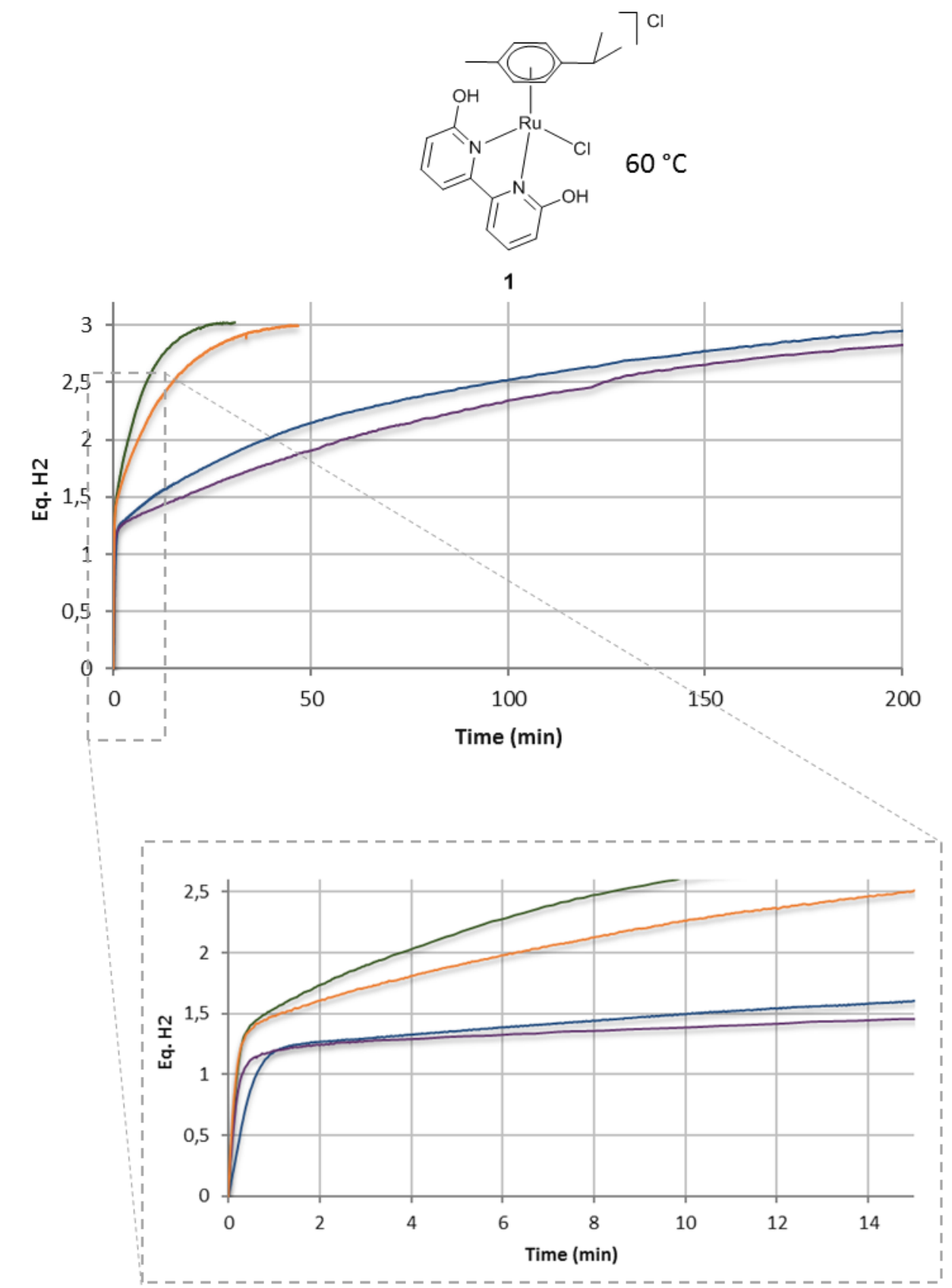

$\mathrm{MeOH} \longrightarrow \mathrm{H}_{2} \mathrm{O} / \mathrm{MeOH}=1 / 9-\mathrm{MeOH} / \mathrm{H}_{2} \mathrm{O}=1 / 1-\mathrm{H}_{2} \mathrm{O}$

Figure S30. Comparative reaction profiles of $A B$ solvolysis using different $\mathrm{MeOH} / \mathrm{H}_{2} \mathrm{O}$ mixtures as solvent and $[\mathrm{Ru}(\mathrm{p}-\mathrm{Cym})(\mathrm{dhbp}) \mathrm{Cl}] \mathrm{Cl}(\mathbf{1})$ as precatalyst. Conditions: $[\mathrm{Ru}]=2.3 \mathrm{mM},[\mathrm{AB}]=0.46$ $\mathrm{M}, 1.5 \mathrm{~mL}$ solvent, $60^{\circ} \mathrm{C}$. 

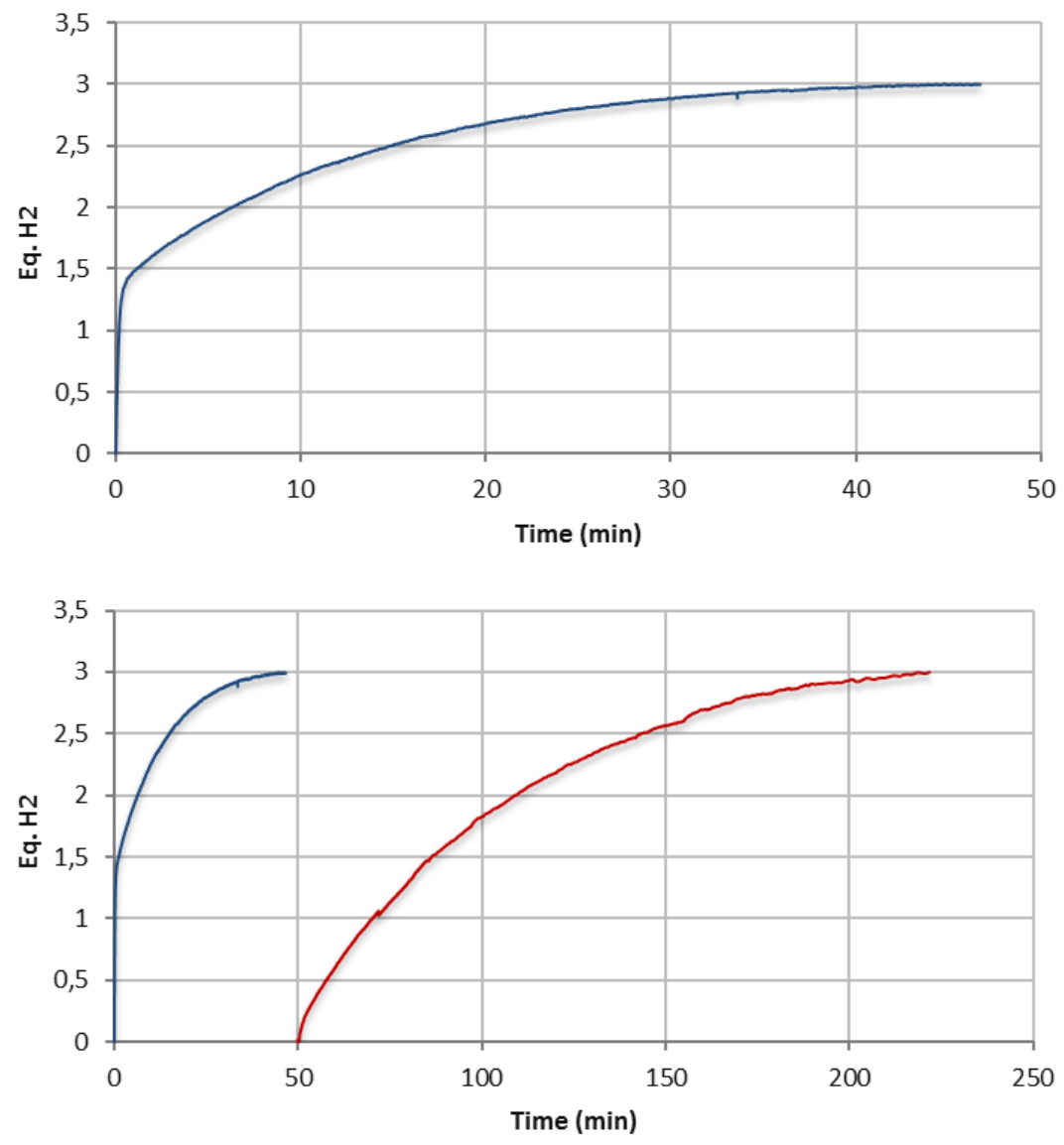

- 1 st addition $\quad$ 2nd addition

Figure S31. Reaction profiles of successive $A B$ solvolysis using $[\mathrm{Ru}(\mathrm{p}-\mathrm{Cym})(\mathrm{dhbp}) \mathrm{Cl}] \mathrm{Cl}(\mathbf{1})$. (Table 2, Entry 13). Conditions: $[\mathrm{Ru}]=2.3 \mathrm{mM},[\mathrm{AB}]=0.46 \mathrm{M}, 1.5 \mathrm{~mL} \mathrm{NaOH} 0.46 \mathrm{M}$ in $\mathrm{MeOH}, 60^{\circ} \mathrm{C}$. BOTTOM: Reaction profiles of successive reactions.

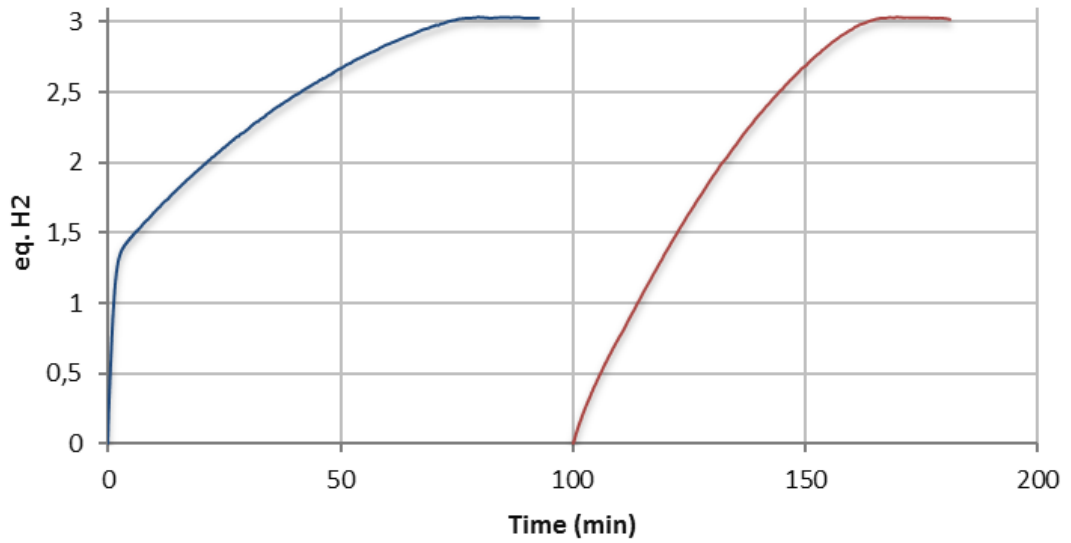

-1 st addition $\quad$ 2nd addition

Figure S32 Reaction profiles of successive $A B$ hydrolysis using [Ru(p-Cym)(dhbp)Cl]Cl (1). (Table 2, Entry 14). Conditions: $[\mathrm{Ru}]=2.3 \mathrm{mM},[\mathrm{AB}]=0.46 \mathrm{M}, 100 \mathrm{mg} \mathrm{NaCl}$-modified molecular sieves, $1.5 \mathrm{~mL} \mathrm{H} \mathrm{H}_{2} \mathrm{O} 6{ }^{\circ} \mathrm{C}$. Additional $21.3 \mathrm{mg}$ of $\mathrm{AB}$ for the consecutive reaction. BOTTOM: Reaction profiles of successive reactions. 

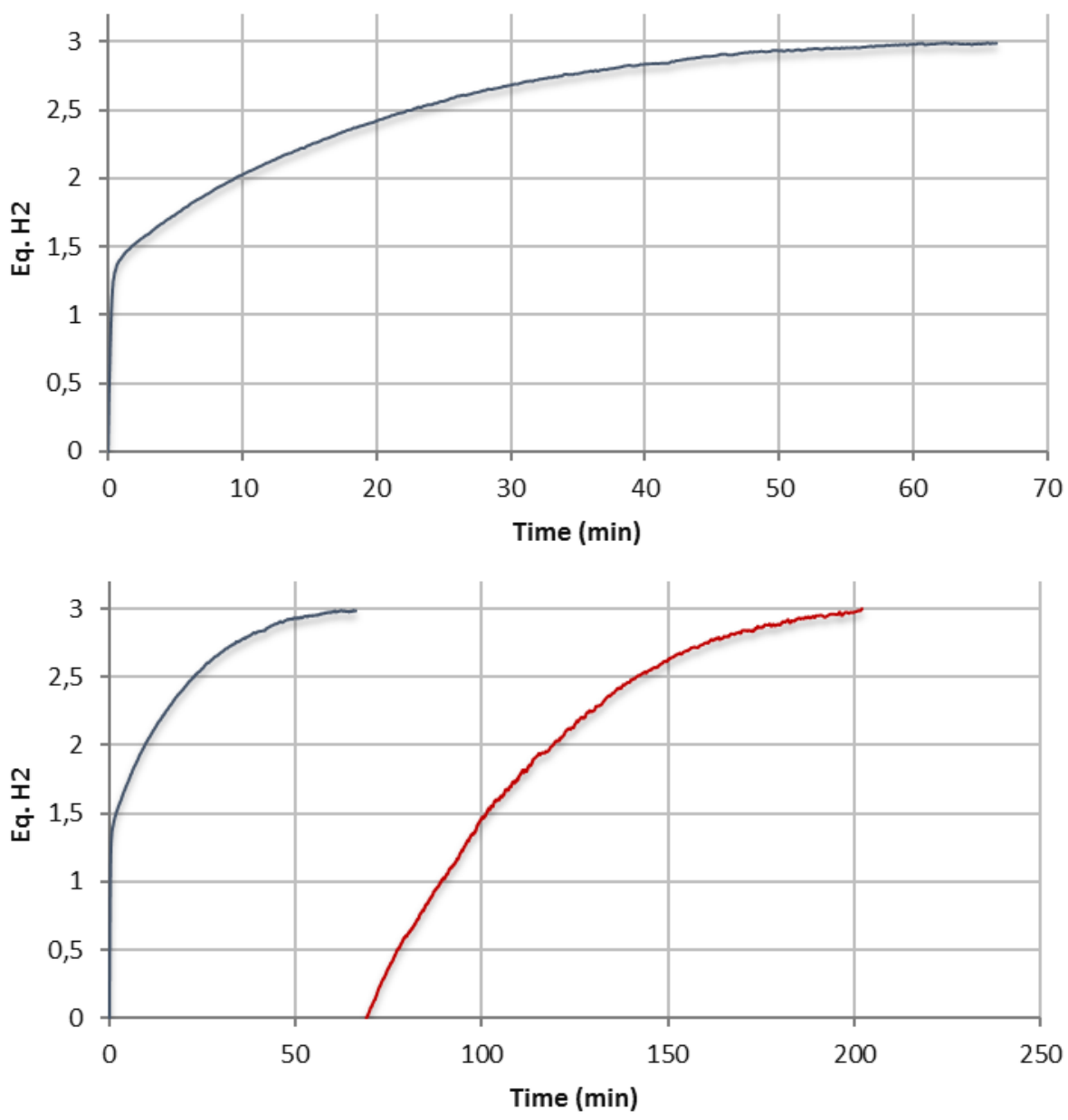

-1st addition $\quad$ 2nd addition

Figure S33. Reaction profiles of successive $A B$ hydrolysis using [Ru(p-Cym)(dhbp)Cl]Cl (1). (Table 2, Entry 15). Conditions: $[\mathrm{Ru}]=2.3 \mathrm{mM},[\mathrm{AB}]=0.46 \mathrm{M}, 200 \mathrm{mg} \mathrm{MgSO}_{4}, 1.5 \mathrm{~mL} \mathrm{MeOH}, 60^{\circ} \mathrm{C}$. BOTTOM: Reaction profiles of successive reactions. 

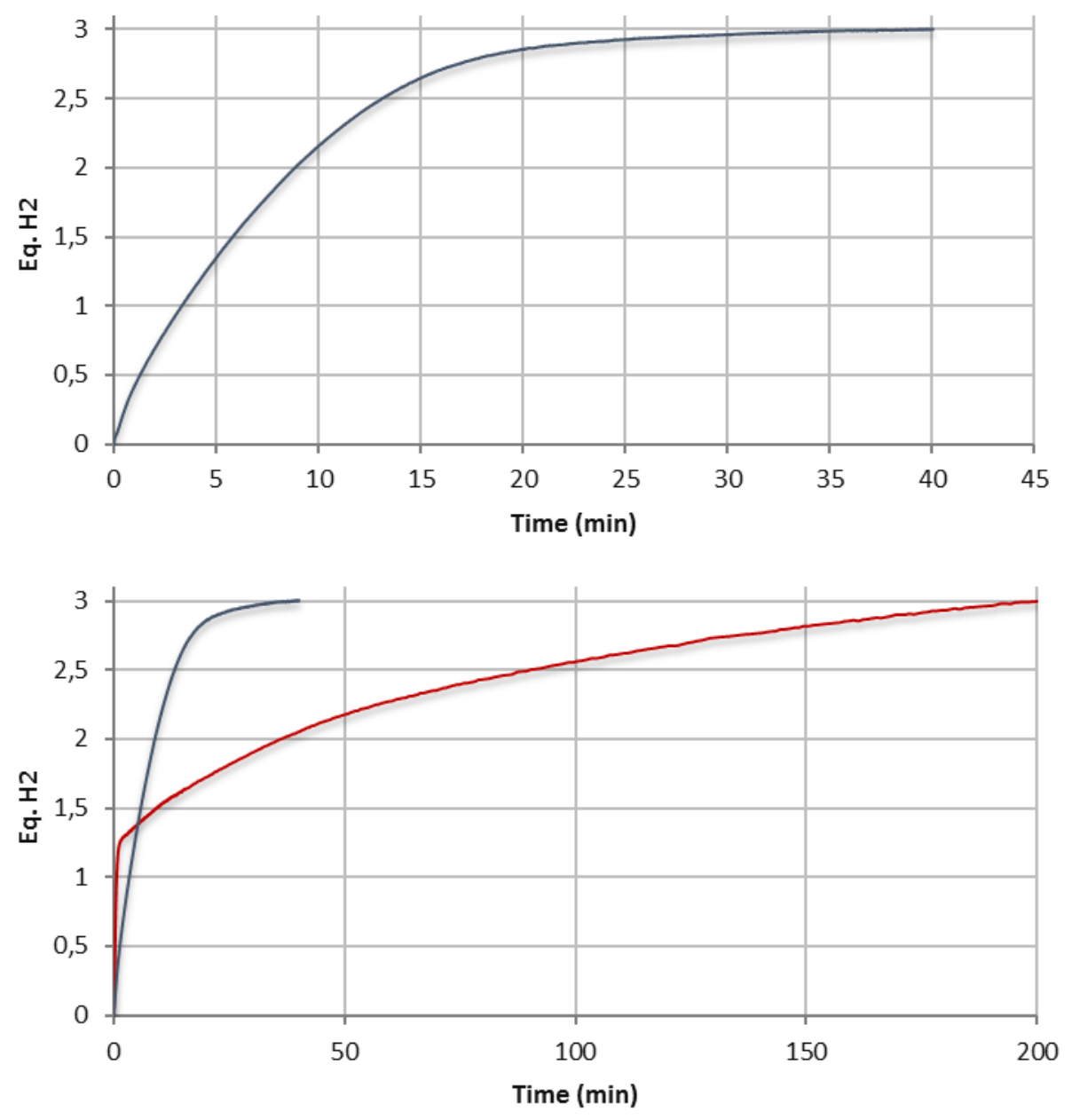

Figure S34. Reaction profiles of: TOP: $\mathrm{HMe}_{2} \mathrm{~N} \cdot \mathrm{BH}_{3}$ (DMAB) hydrolysis using $[\mathrm{Ru}(\mathrm{p}-$ Cym) $($ dhbp $) \mathrm{Cl}] \mathrm{Cl}(\mathbf{1})$. (Table S1, Entry 1). Conditions: $[\mathrm{Ru}]=2.3 \mathrm{mM},[D M A B]=0.46 \mathrm{M}, 1.5 \mathrm{~mL}$ $\mathrm{H}_{2} \mathrm{O}, 60^{\circ} \mathrm{C}$. BOTTOM: Comparison of DMAB and $\mathrm{AB}$ hydrolysis using $[\mathrm{Ru}(\mathrm{p}-\mathrm{Cym})(\mathrm{dhbp}) \mathrm{Cl}] \mathrm{Cl}(\mathbf{1})$ as precatalyst.

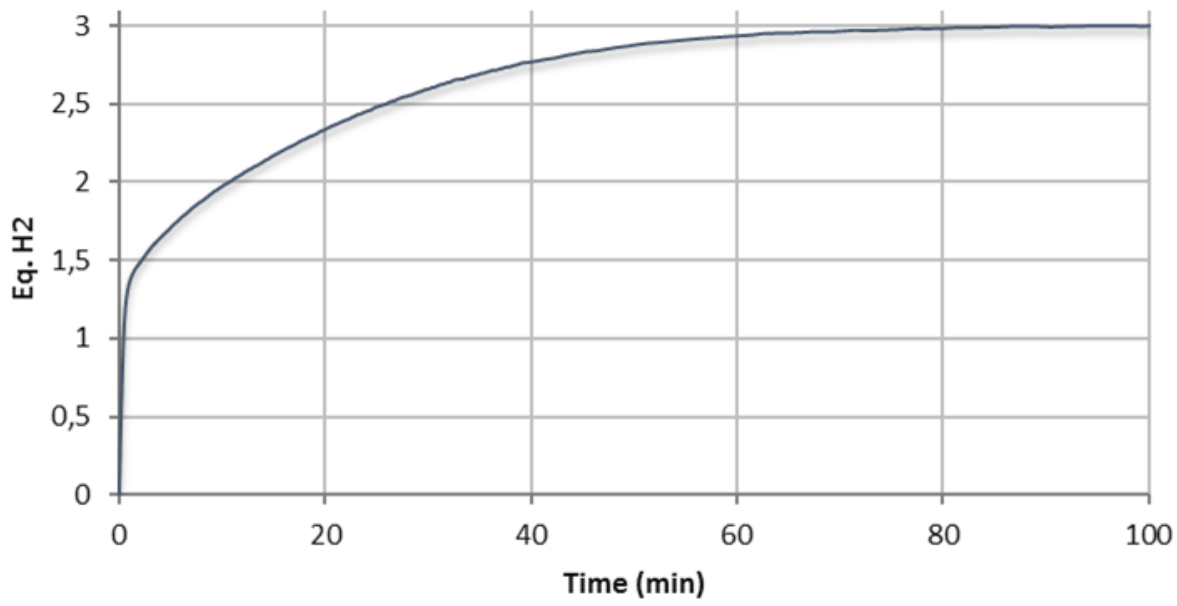

Figure S35. Reaction profile of DMAB alcoholysis using $[\mathrm{Ru}(\mathrm{p}-\mathrm{Cym})(\mathrm{dhbp}) \mathrm{Cl}] \mathrm{Cl}(\mathbf{1})$. (Table S1, Entry 2). Conditions: $[\mathrm{Ru}]=2.3 \mathrm{mM},[\mathrm{DMAB}]=0.46 \mathrm{M}, 1.5 \mathrm{~mL} \mathrm{MeOH}, 60^{\circ} \mathrm{C}$. 


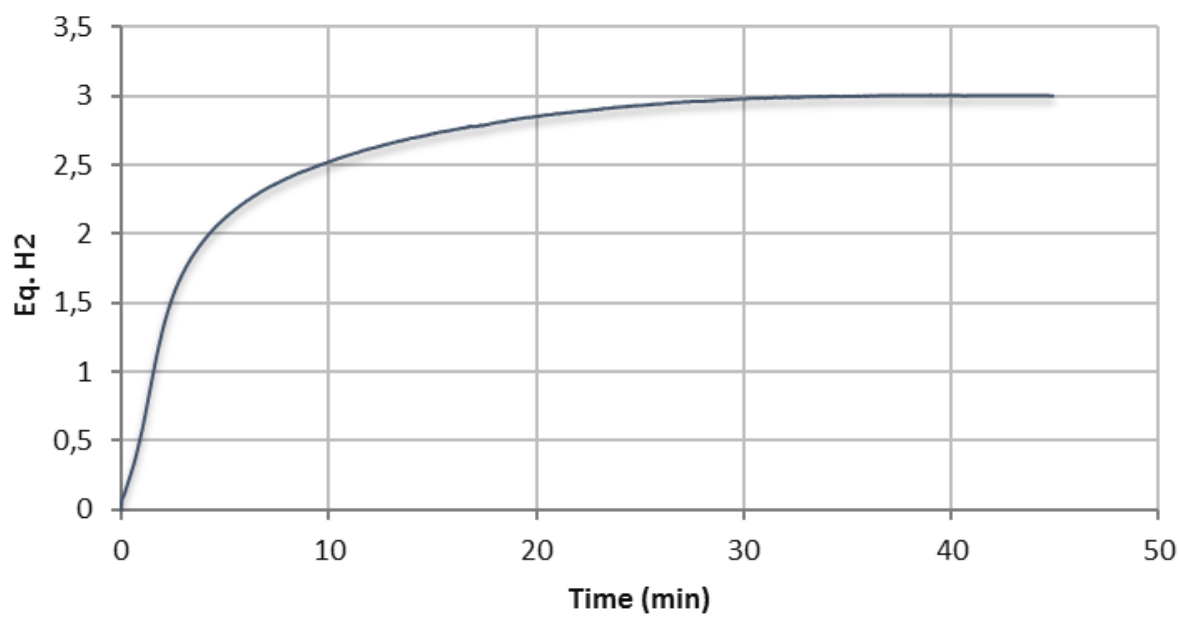

Figure S36. Reaction profile of DMAB hydrolysis using [Ru(p-Cym)(dhbp)Cl]Cl (1). (Table S1, Entry 3). Conditions: $[\mathrm{Ru}]=2.3 \mathrm{mM},\left[\mathrm{HMe}_{2} \mathrm{~N} \cdot \mathrm{BH}_{3}\right]=[\mathrm{NaOH}]=0.46 \mathrm{M}, 1.5 \mathrm{~mL} \mathrm{H} \mathrm{H}_{2}, 60^{\circ} \mathrm{C}$.

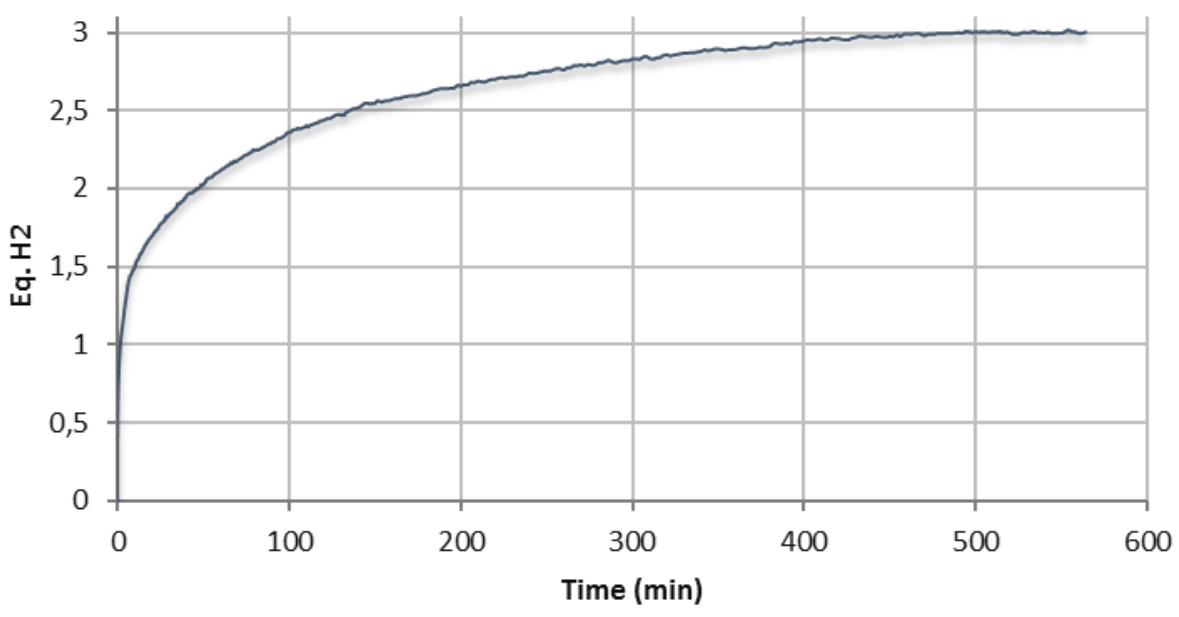

Figure S37. Reaction profile of DMAB alcoholysis using $[\mathrm{Ru}(\mathrm{p}-\mathrm{Cym})(\mathrm{dhbp}) \mathrm{Cl}] \mathrm{Cl}(\mathbf{1})$. (Table S1, Entry 4). Conditions: $[\mathrm{Ru}]=2.3 \mathrm{mM},[\mathrm{DMAB}]=[\mathrm{NaOH}]=0.46 \mathrm{M}, 1.5 \mathrm{~mL} \mathrm{MeOH}, 60^{\circ} \mathrm{C}$.

Table S1. Catalytic results. DMAB solvolysis using precatalysts 1.

\begin{tabular}{ccccc}
\hline Entry & Solvent & TOF $_{10 \%}\left(\mathrm{~h}^{-1}\right)$ & TOF $_{50 \%}\left(\mathrm{~h}^{-1}\right)$ & Total time \\
\hline 1 & $\mathrm{H}_{2} \mathrm{O}$ & 1627 & 1032 & $35 \mathrm{~min}$ \\
2 & $\mathrm{MeOH}$ & 12414 & 2775 & $80 \mathrm{~min}$ \\
$3^{[\mathrm{b}]}$ & $\mathrm{NaOH} / \mathrm{H}_{2} \mathrm{O}$ & 2239 & 2521 & $35 \mathrm{~min}$ \\
$4^{[\mathrm{b}]}$ & $\mathrm{NaOH} / \mathrm{MeOH}$ & 11921 & 589 & $3 \mathrm{~h}$
\end{tabular}

Reaction conditions: $[\mathrm{cat}]=2.3 \cdot 10^{-3} \mathrm{M},[\mathrm{DMAB}]=0.46 \mathrm{M}, 1.5 \mathrm{~mL}$ solvent, $60^{\circ} \mathrm{C}$. $[\mathrm{a}]$ Time to reach full conversion. $[\mathrm{b}][\mathrm{NaOH}]=$ $0.46 \mathrm{M}$. 


\section{Supporting Information}

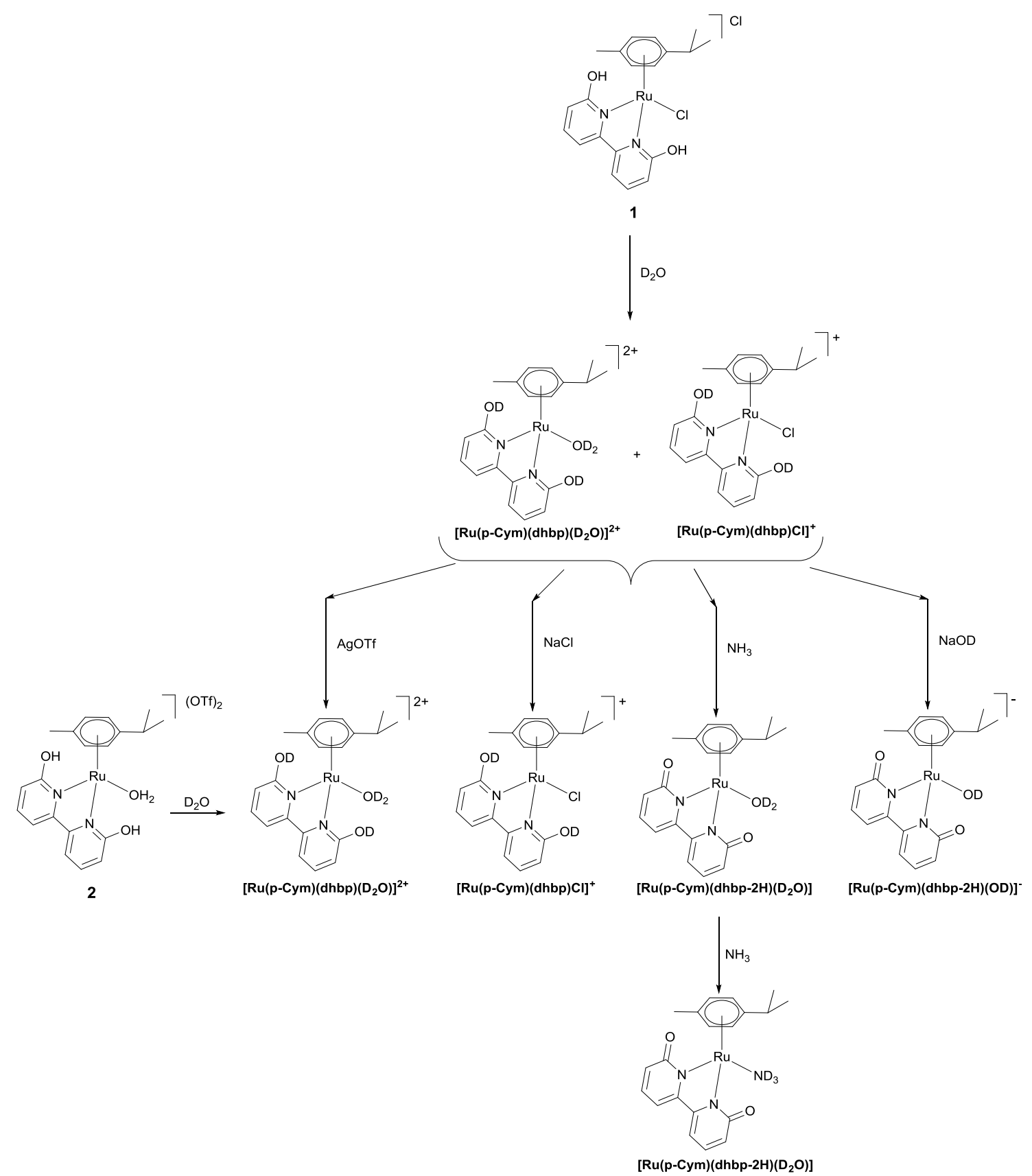

Scheme S1. Reactivity scheme used for the in situ characterizations in $\mathrm{D}_{2} \mathrm{O}$. 


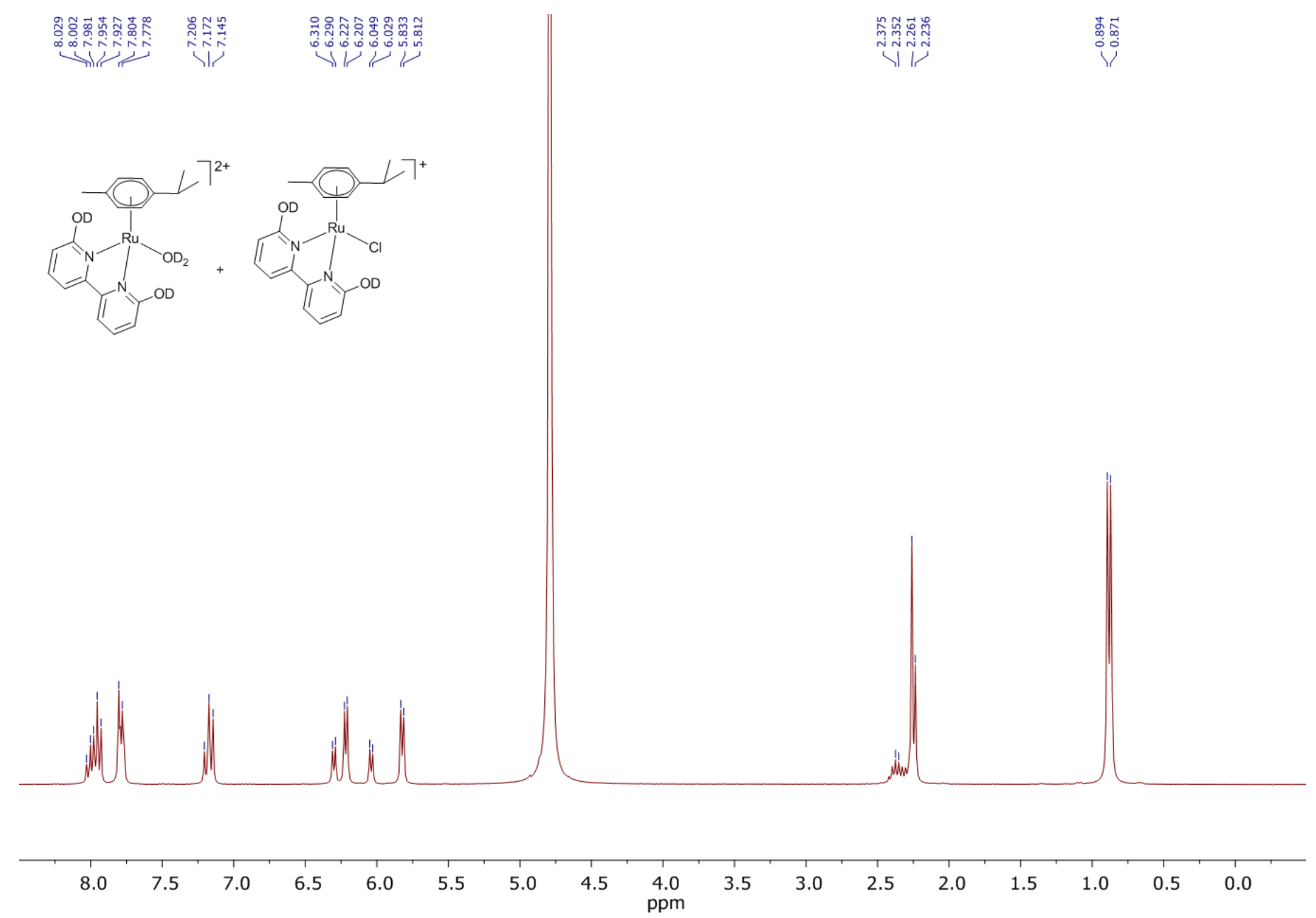

Figure S38. ${ }^{1} \mathrm{H}$ NMR spectra (300 MHz). $3.89 \mathrm{mg}$ of $[\mathrm{Ru}(\mathrm{p}-\mathrm{Cym})(\mathrm{dhbp}) \mathrm{Cl}] \mathrm{Cl}(\mathbf{1})$ in $0.5 \mathrm{~mL}$ of $\mathrm{D}_{2} \mathrm{O}$. 


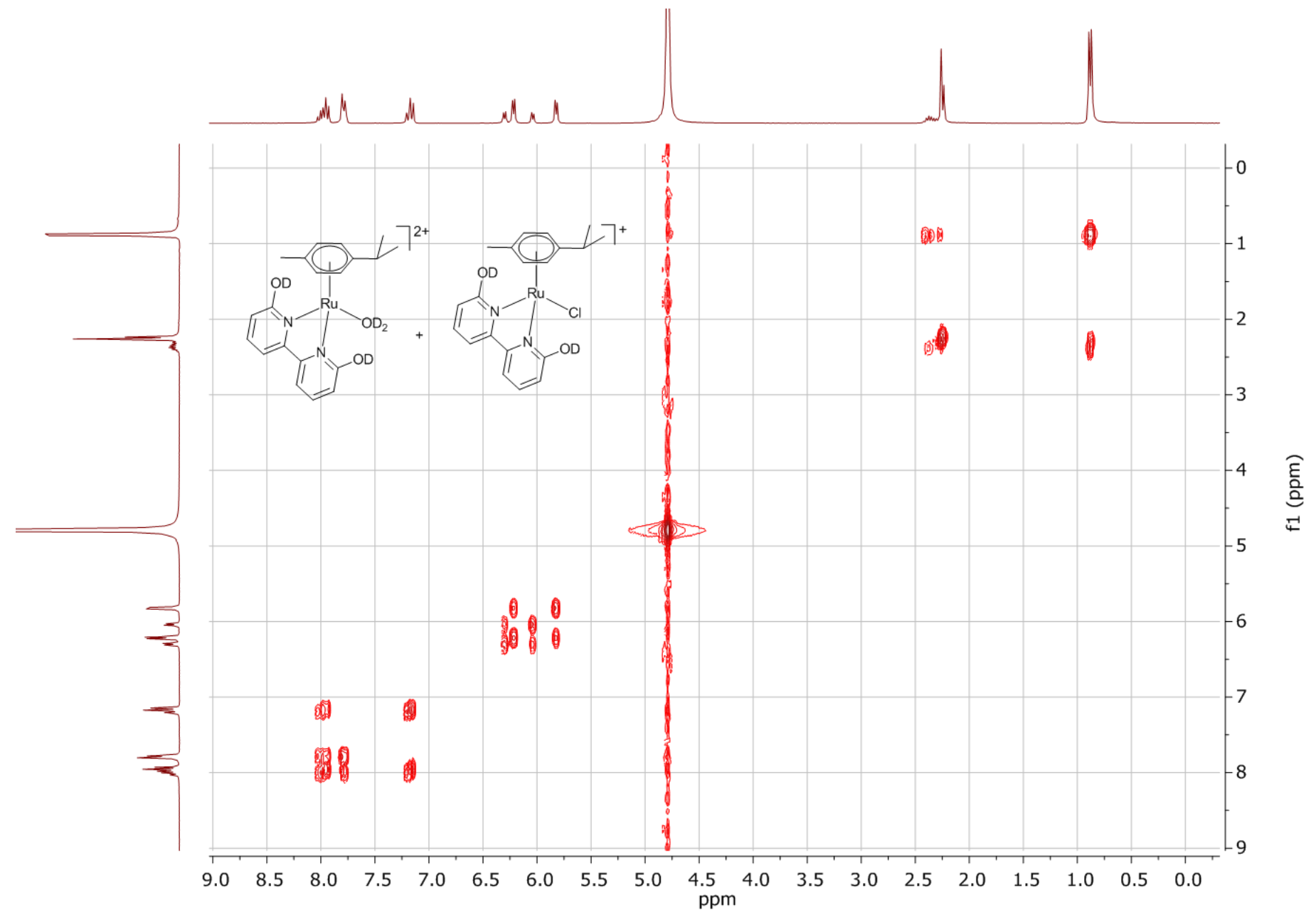

Figure S39. COSY NMR spectra (300 MHz). $3.89 \mathrm{mg}$ of $[\mathrm{Ru}(\mathrm{p}-\mathrm{Cym})(\mathrm{dhbp}) \mathrm{Cl}] \mathrm{Cl}(\mathbf{1})$ in $0.5 \mathrm{~mL}$ of $\mathrm{D}_{2} \mathrm{O}$. 
In situ formation and characterization of $[\mathrm{Ru}(\mathrm{p}-\mathrm{Cym})(\mathrm{dhbp}) \mathrm{Cl}]^{+}\left(\mathrm{D}_{2} \mathrm{O}\right)$ :

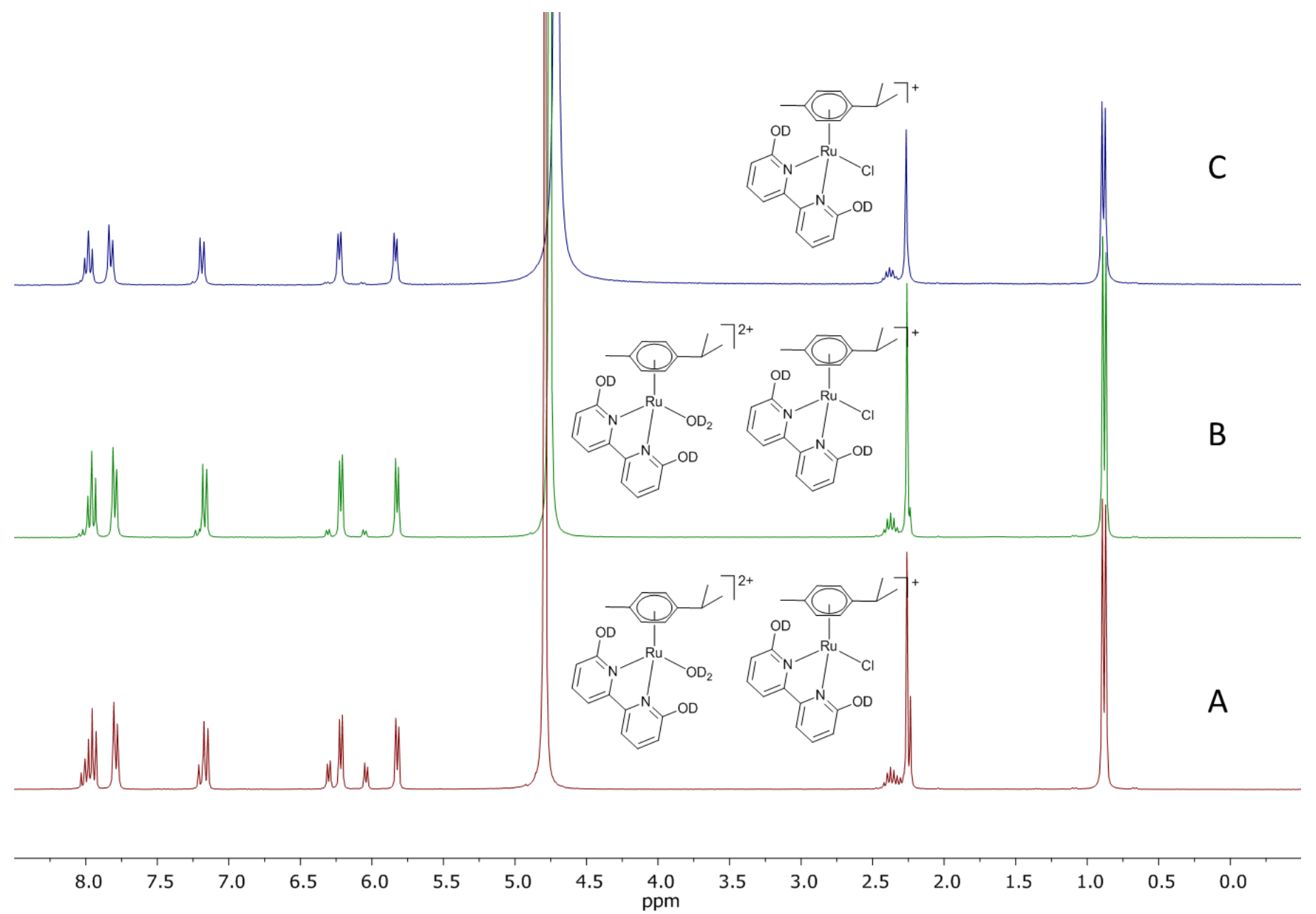

Figure S40. ${ }^{1} \mathrm{H}$ NMR spectra (300 MHz). $3.16 \mathrm{mg}$ of $[\mathrm{Ru}(\mathrm{p}-\mathrm{Cym})(\mathrm{dhbp}) \mathrm{Cl}] \mathrm{Cl}(\mathbf{1})$ and in $0.5 \mathrm{~mL}$ of $\mathrm{D}_{2} \mathrm{O}$. B: After addition of $10 \mathrm{mg}$ of $\mathrm{NaCl}$. B: After addition of 15 $\mathrm{mg}$ more of $\mathrm{NaCl}$. 
Supporting Information

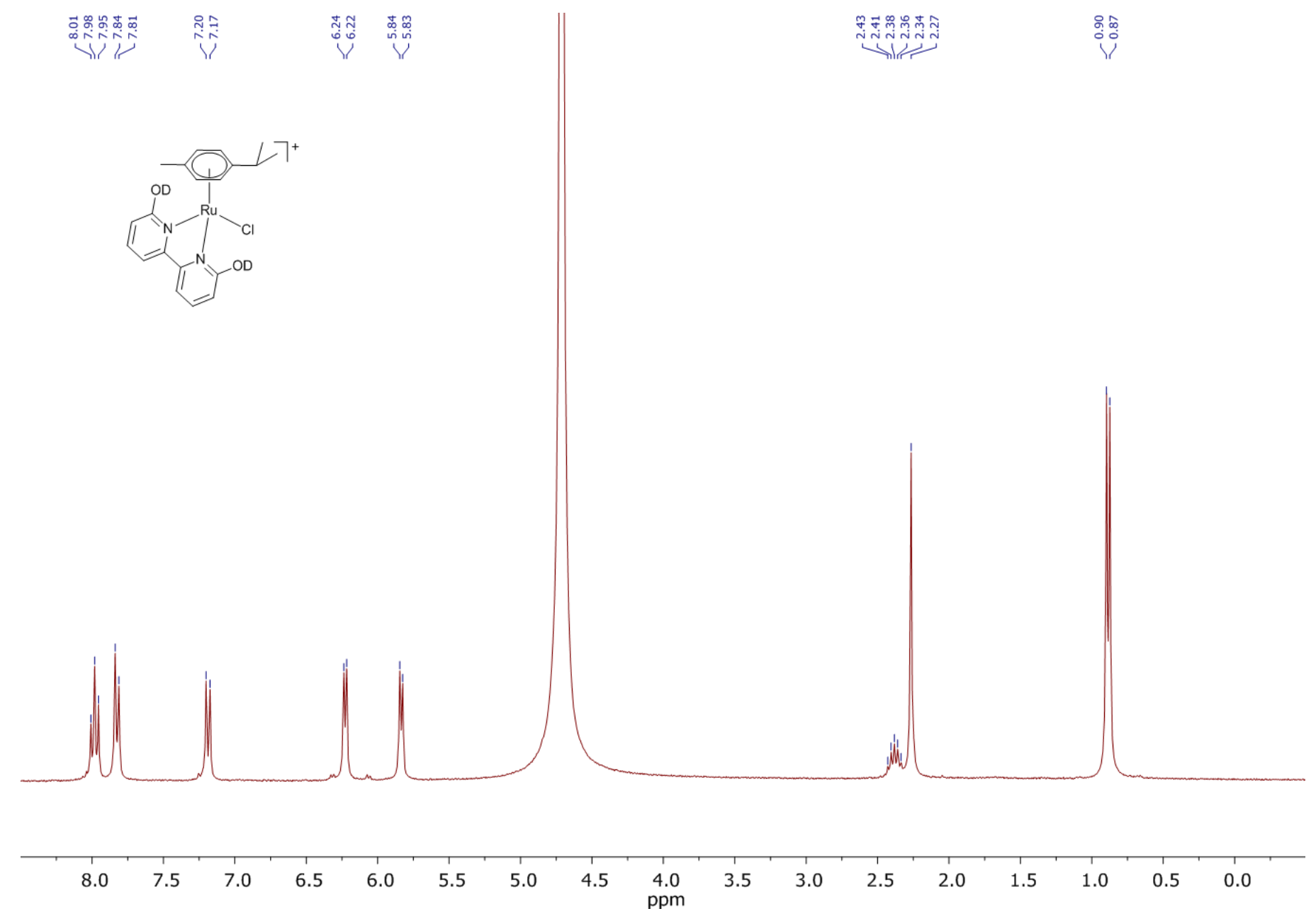

Figure S41. ${ }^{1} \mathrm{H}$ NMR spectra (300 MHz). $3.16 \mathrm{mg}$ of $[\mathrm{Ru}(\mathrm{p}-\mathrm{Cym})(\mathrm{dhbp}) \mathrm{Cl}] \mathrm{Cl}(\mathbf{1})$ in $0.5 \mathrm{~mL}$ of $\mathrm{D}_{2} \mathrm{O}$, after addition of $25 \mathrm{mg}$ of $\mathrm{NaCl}$. 

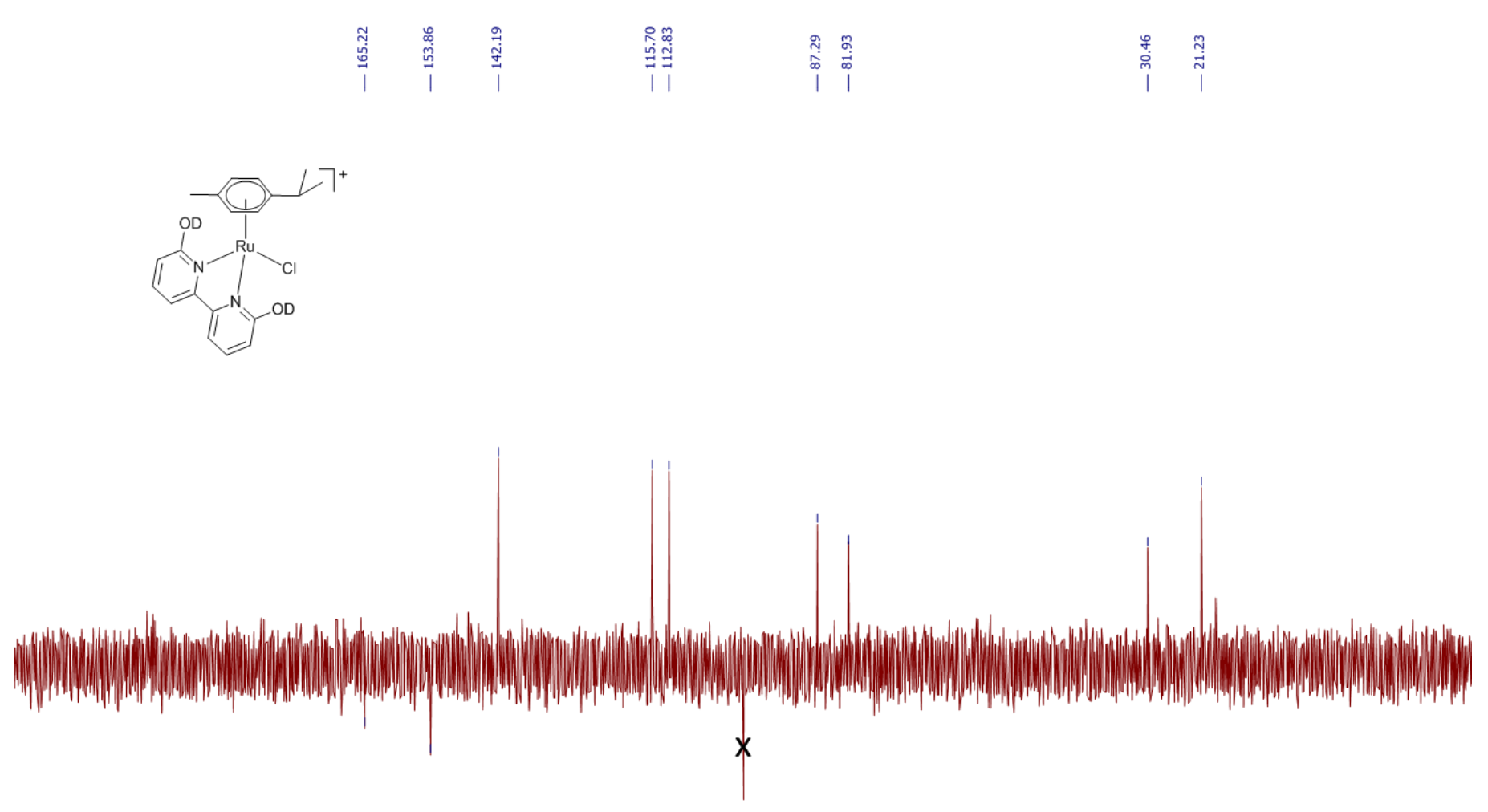

$\begin{array}{lllllllllllllllllllllllll}220 & 210 & 200 & 190 & 180 & 170 & 160 & 150 & 140 & 130 & 120 & 110 & 100 & 90 & 80 & 70 & 60 & 50 & 40 & 30 & 20 & 10 & 0 & -10 & -20\end{array}$

Figure S42. ${ }^{13} \mathrm{C}(\mathrm{APT}) \mathrm{NMR}$ spectra $(75 \mathrm{MHz}) .3 .16 \mathrm{mg}$ of $[\mathrm{Ru}(\mathrm{p}-\mathrm{Cym})(\mathrm{dhbp}) \mathrm{Cl}] \mathrm{Cl}(\mathbf{1})$ in $0.5 \mathrm{~mL}$ of $\mathrm{D}_{2} \mathrm{O}$, after addition of $25 \mathrm{mg}$ of $\mathrm{NaCl}$. One of the quaternary carbons of $\mathrm{p}$-Cymene is hidden in the background noise of the spectra. Peak at $100 \mathrm{ppm}$ is an artifact (center of the acquisition window). 


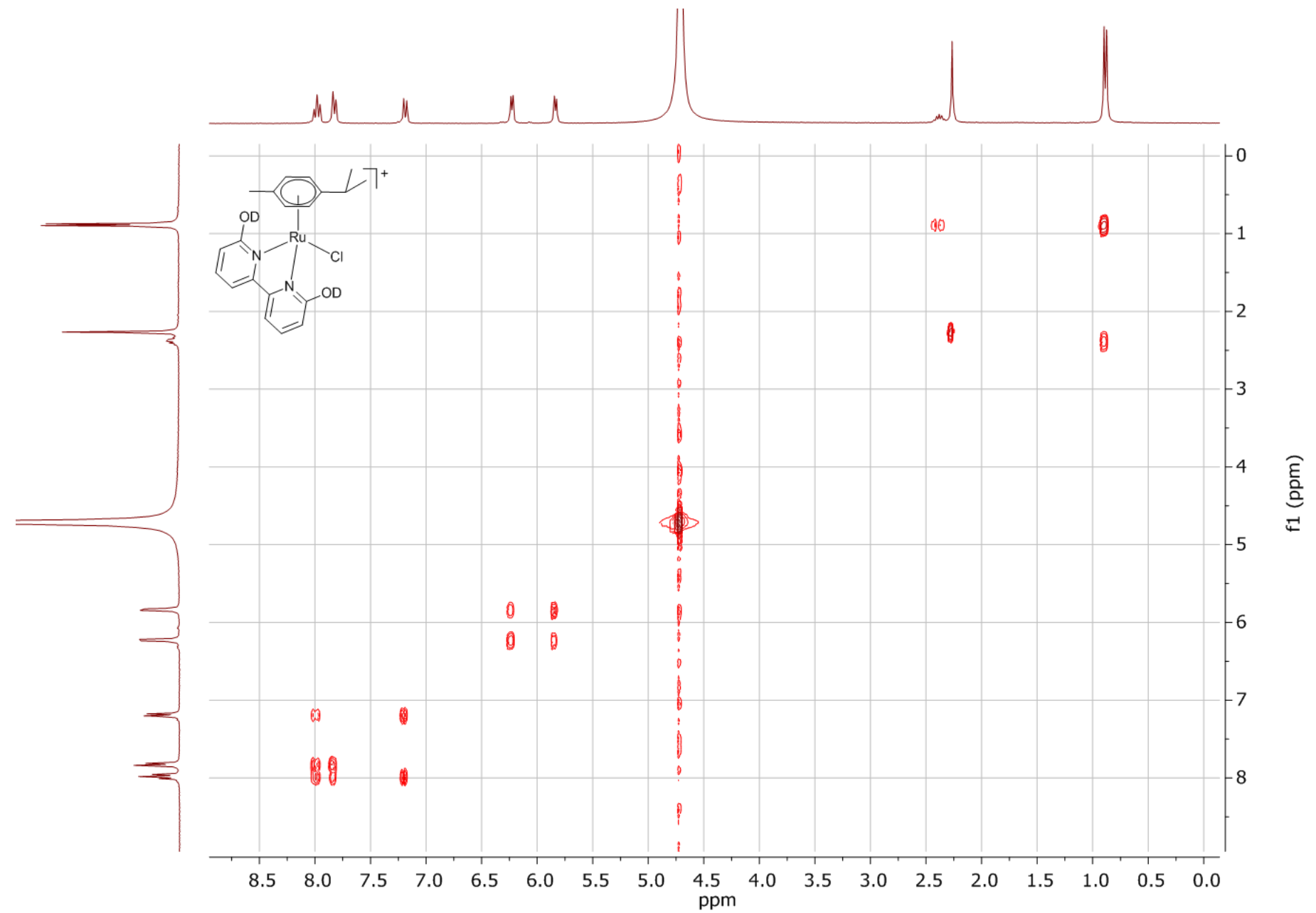

Figure S43. COSY NMR spectra (300 MHz). $3.16 \mathrm{mg}$ of $[\mathrm{Ru}(\mathrm{p}-\mathrm{Cym})(\mathrm{dhbp}) \mathrm{Cl}] \mathrm{Cl}(\mathbf{1})$ in $0.5 \mathrm{~mL}$ of $\mathrm{D}_{2} \mathrm{O}$, after addition of $25 \mathrm{mg}$ of $\mathrm{NaCl}$. 


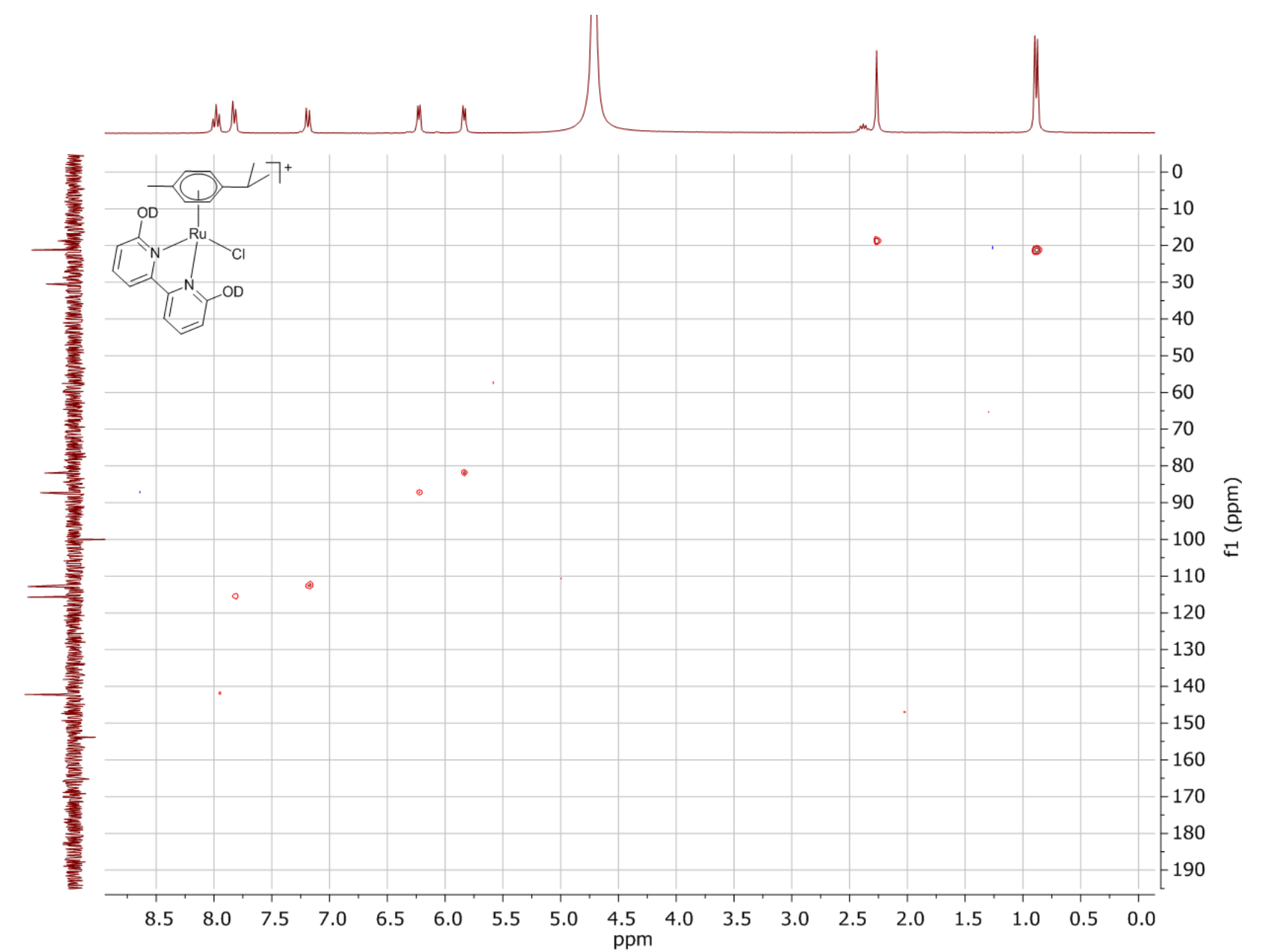

Figure S44. HSQC NMR spectra (300 MHz). $3.16 \mathrm{mg}$ of $[\mathrm{Ru}(\mathrm{p}-\mathrm{Cym})(\mathrm{dhbp}) \mathrm{Cl}] \mathrm{Cl}(\mathbf{1})$ in $0.5 \mathrm{~mL}$ of $\mathrm{D}_{2} \mathrm{O}$, after addition of $25 \mathrm{mg}$ of $\mathrm{NaCl}$. 
In situ formation and characterization of $\left[\operatorname{Ru}(\mathrm{p}-\mathrm{Cym})(\mathrm{dhbp})\left(\mathrm{D}_{2} \mathrm{O}\right)\right]^{2+}\left(\mathrm{D}_{2} \mathrm{O}\right)$ :

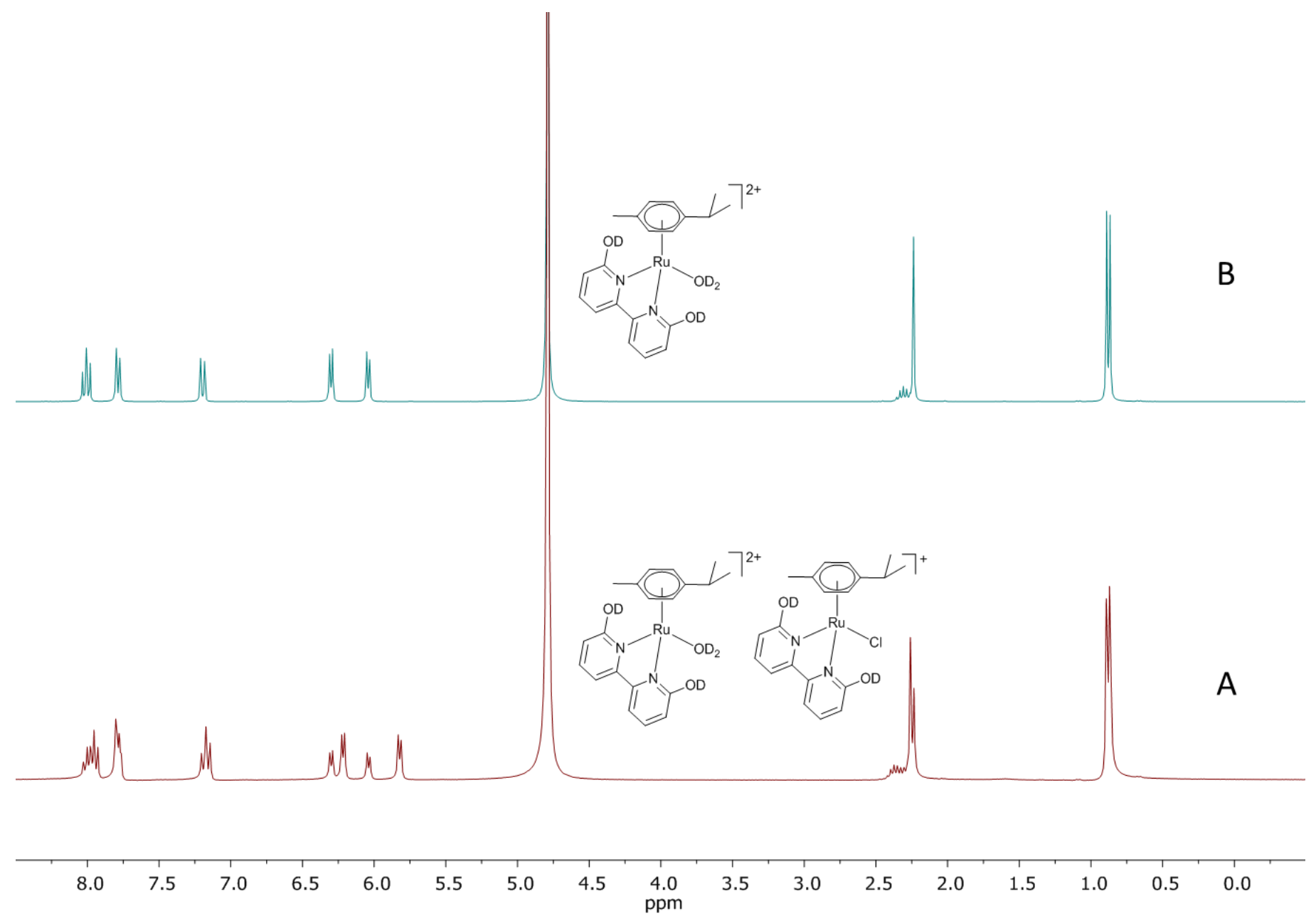

Figure S45. ${ }^{1} \mathrm{H}$ NMR spectra (300 MHz). $3.65 \mathrm{mg}$ of $[\mathrm{Ru}(\mathrm{p}-\mathrm{Cym})(\mathrm{dhbp}) \mathrm{Cl}] \mathrm{Cl}(\mathbf{1})$ and in $0.5 \mathrm{~mL}$ of $\mathrm{D}_{2} \mathrm{O}$. B: After addition of $10 \mathrm{mg}$ of AgOTf. 


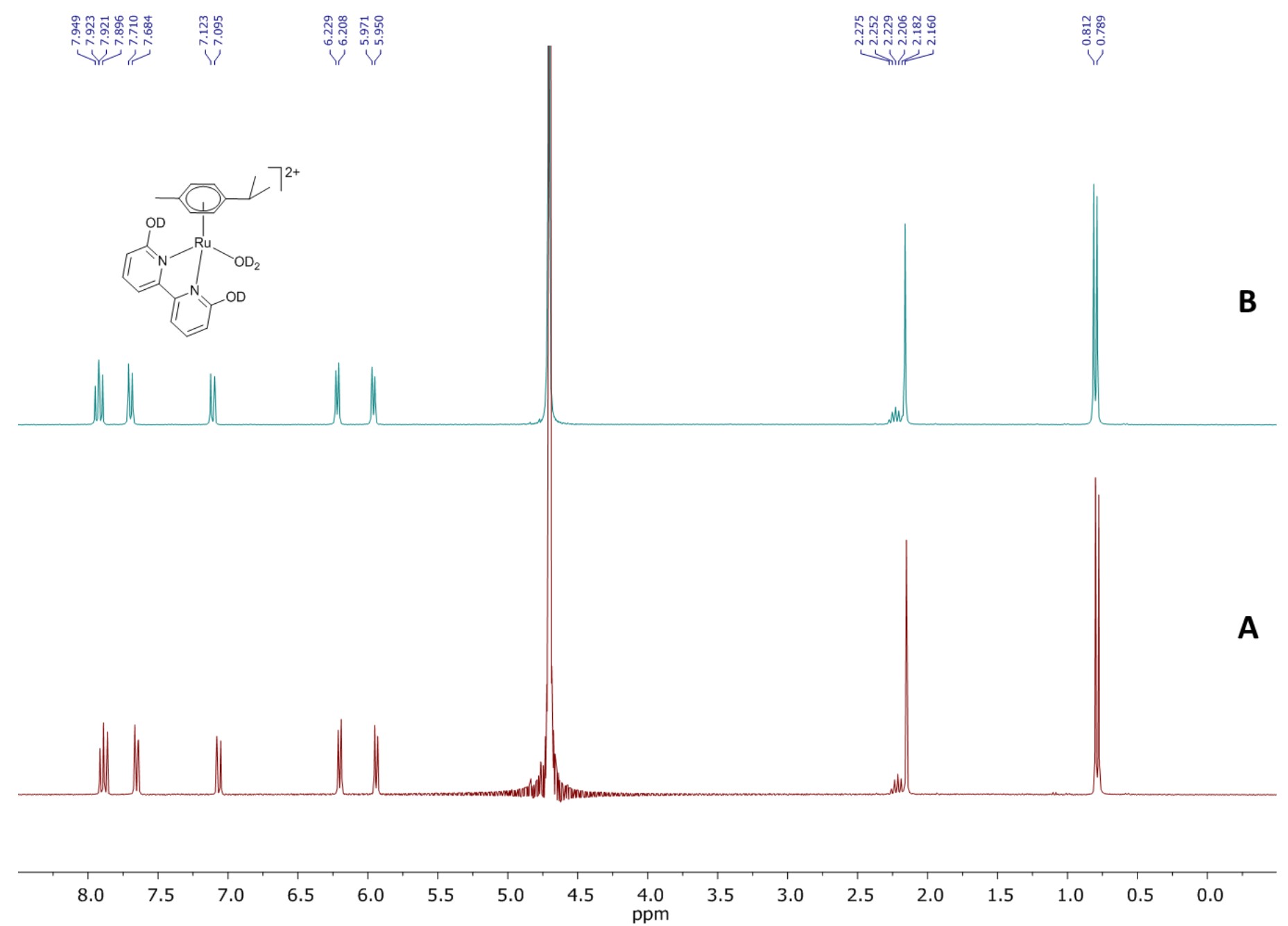

Figure S46. ${ }^{1} \mathrm{H}$ NMR spectra (300 MHz). A. $1.24 \mathrm{mg}$ of $\left[\mathrm{Ru}(\mathrm{p}-\mathrm{Cym})(\mathrm{dhbp})\left(\mathrm{H}_{2} \mathrm{O}\right)\right](\mathrm{OTf})_{2}(\mathbf{2})$ in $0.5 \mathrm{~mL}$ of $\mathrm{D}_{2} \mathrm{O}$. B. $3.65 \mathrm{mg}$ of $[\mathrm{Ru}(\mathrm{p}-\mathrm{Cym})(\mathrm{dhbp}) \mathrm{Cl}] \mathrm{Cl}(\mathbf{1})$ in $0.5 \mathrm{~mL}$ of $\mathrm{D}_{2} \mathrm{O}$, after addition of $10 \mathrm{mg}$ of AgOTf. 


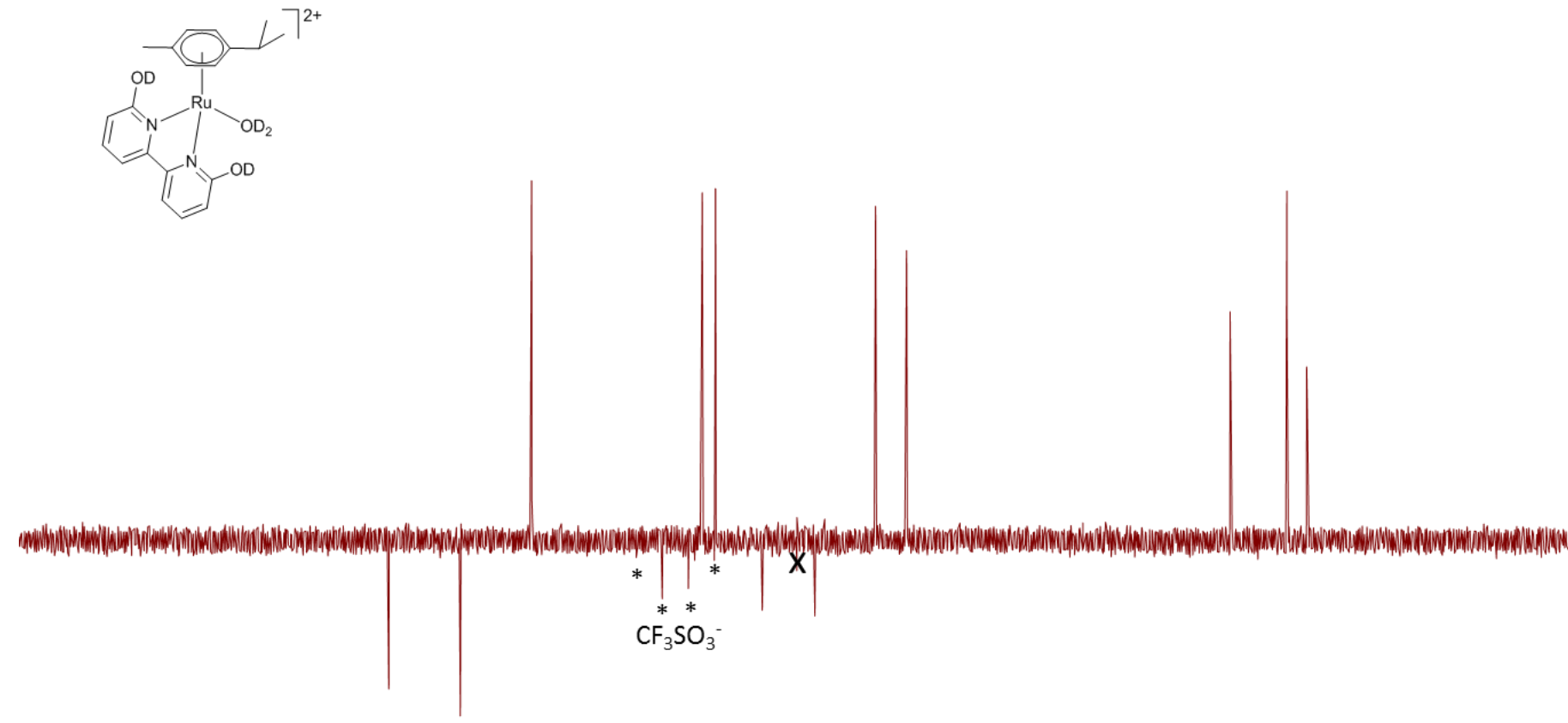

$\begin{array}{lllllllllllllllllllllllll}220 & 210 & 200 & 190 & 180 & 170 & 160 & 150 & 140 & 130 & 120 & 110 & 100 & 90 & 80 & 70 & 60 & 50 & 40 & 30 & 20 & 10 & 0 & -10 & -20\end{array}$

Figure S47. ${ }^{13} \mathrm{C}(\mathrm{APT}) \mathrm{NMR}$ spectra $(75 \mathrm{MHz}) .3 .65 \mathrm{mg}$ of $[\mathrm{Ru}(\mathrm{p}-\mathrm{Cym})(\mathrm{dhbp}) \mathrm{Cl}] \mathrm{Cl}(\mathbf{1})$ in $0.5 \mathrm{~mL}$ of $\mathrm{D}_{2} \mathrm{O}$, after addition of $10 \mathrm{mg}$ of AgOTf. Peak at $100 \mathrm{ppm}$ is an artifact (center of the acquisition window). 


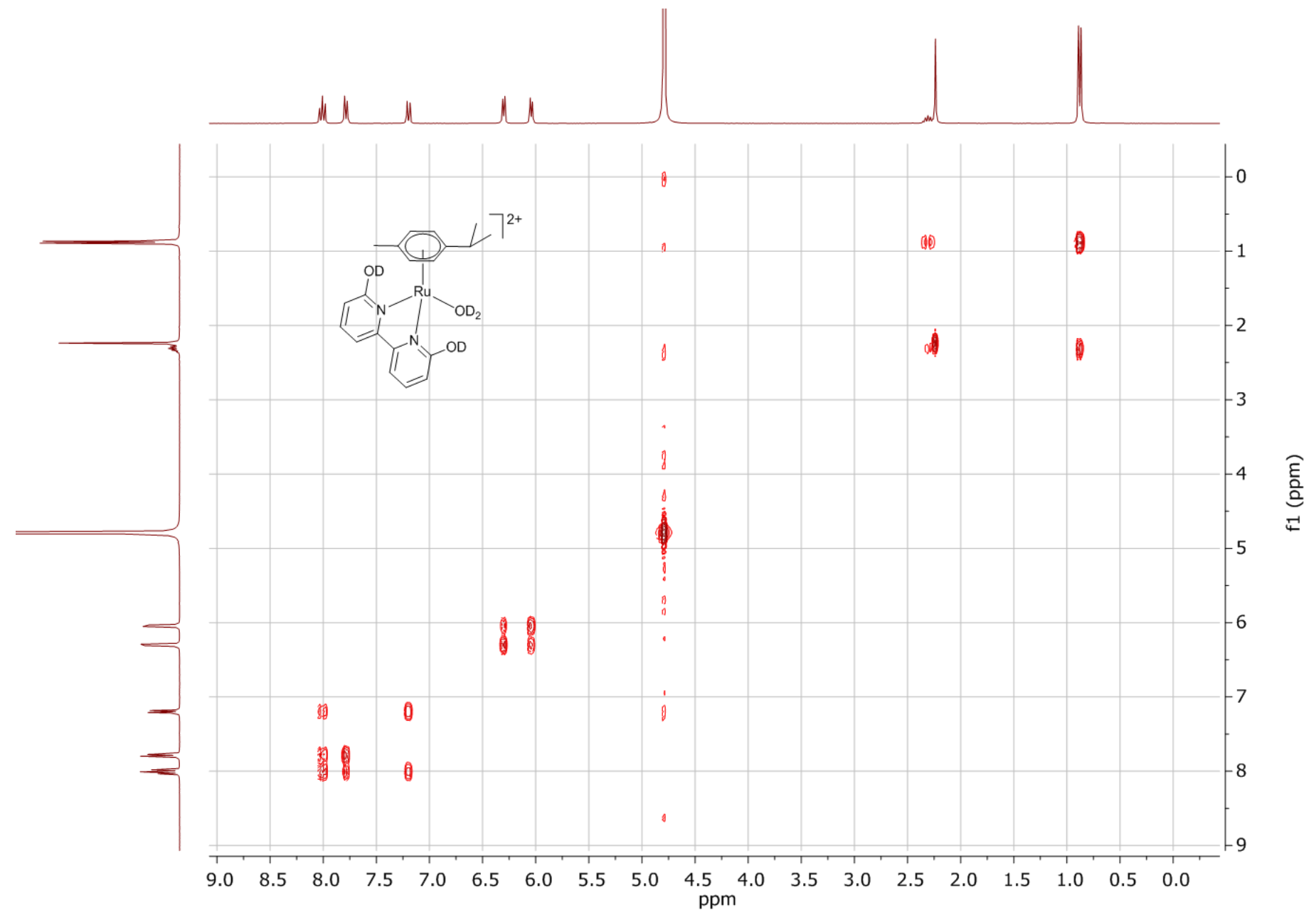

Figure S48. COSY NMR spectra (300 MHz). $3.65 \mathrm{mg}$ of $[\mathrm{Ru}(\mathrm{p}-\mathrm{Cym})(\mathrm{dhbp}) \mathrm{Cl}] \mathrm{Cl}(\mathbf{1})$ in $0.5 \mathrm{~mL}$ of $\mathrm{D}_{2} \mathrm{O}$, after addition of $10 \mathrm{mg}$ of AgOTf. 


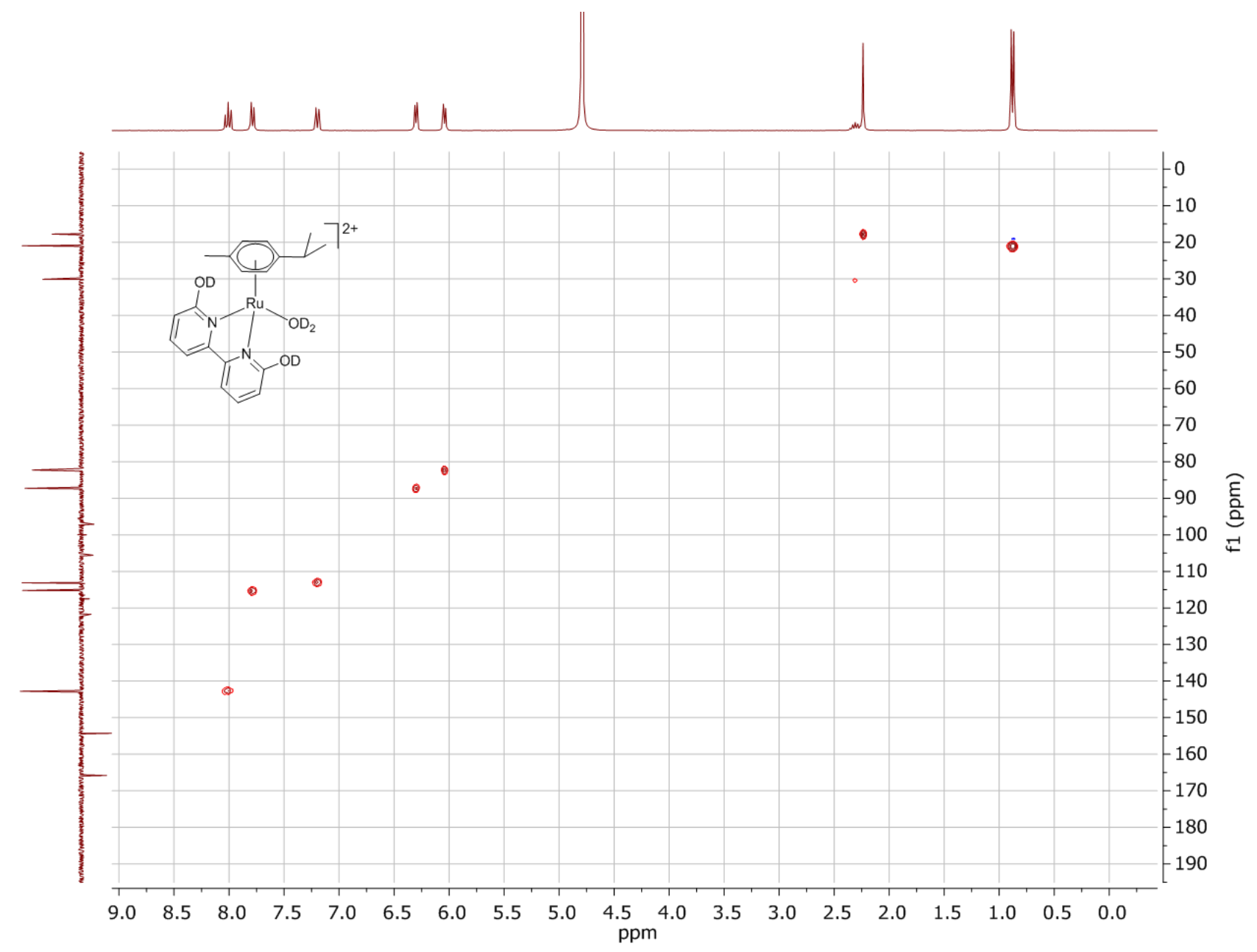

Figure S49 HSQC NMR spectra (300 MHz). $3.65 \mathrm{mg}$ of $[\mathrm{Ru}(\mathrm{p}-\mathrm{Cym})(\mathrm{dhbp}) \mathrm{Cl}] \mathrm{Cl}(\mathbf{1})$ in $0.5 \mathrm{~mL}$ of $\mathrm{D}_{2} \mathrm{O}$, after addition of $10 \mathrm{mg}$ of AgOTf. 
In situ formation and characterization of $\left[\mathrm{Ru}(\mathrm{p}-\mathrm{Cym})(\mathrm{dhbp}-2 \mathrm{H})\left(\mathrm{ND}_{3}\right)\right]\left(\mathrm{D}_{2} \mathrm{O}\right)$ :

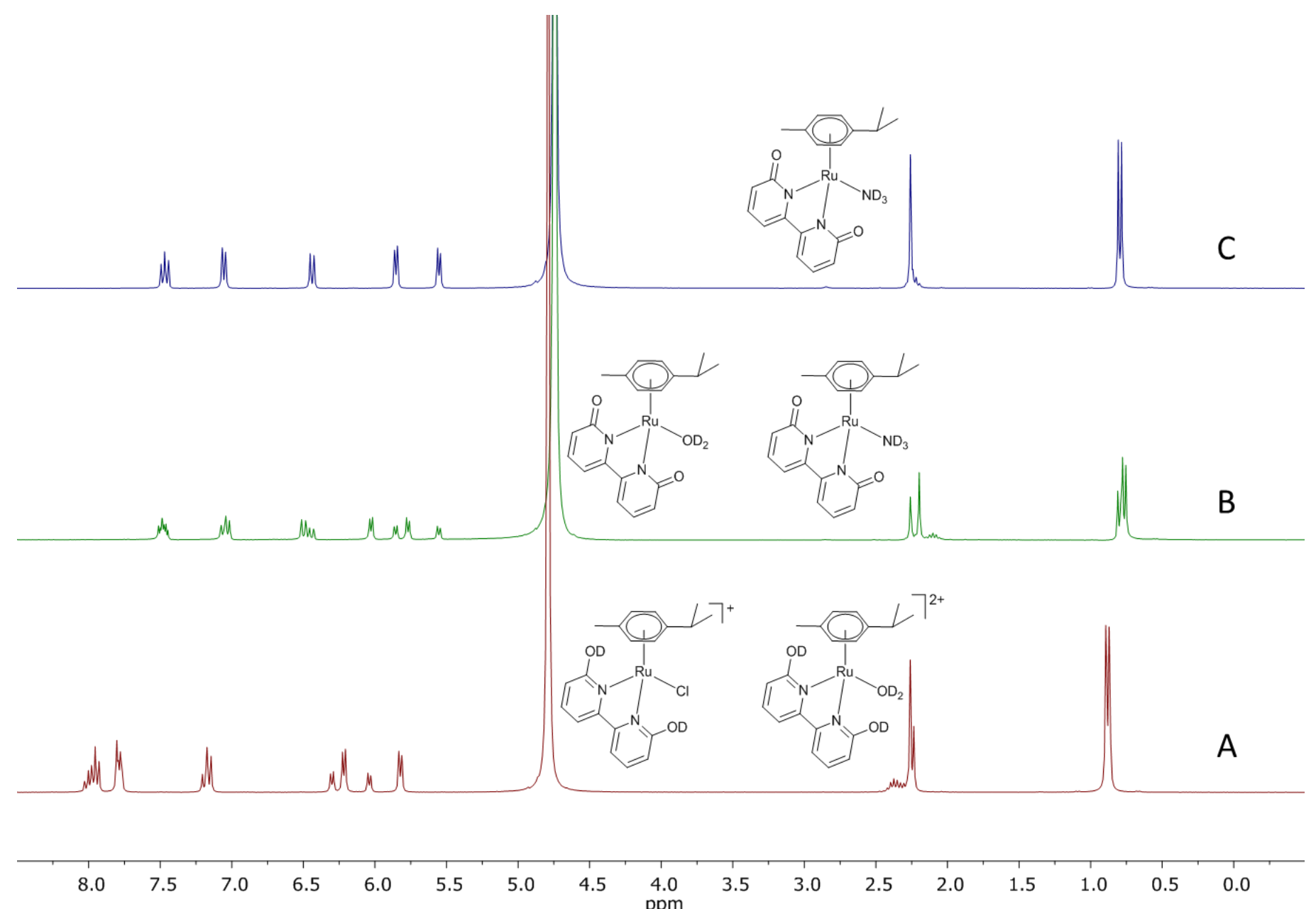

Figure S50. ${ }^{1} \mathrm{H}$ NMR spectra (300 MHz). $3.89 \mathrm{mg}$ of $[\mathrm{Ru}(\mathrm{p}-\mathrm{Cym})(\mathrm{dhbp}) \mathrm{Cl}] \mathrm{Cl}(\mathbf{1})$ in $0.5 \mathrm{~mL}$ of $\mathrm{D}_{2} \mathrm{O}$. B: After addition of $10 \mu \mathrm{L}$ of $\mathrm{NH}_{3}\left(20 \% \mathrm{v} / \mathrm{v}\right.$ in $\left.\mathrm{H}_{2} \mathrm{O}\right)$. C: After addition of $10 \mu \mathrm{L}$ more of $\mathrm{NH}_{3}\left(20 \% \mathrm{v} / \mathrm{v}\right.$ in $\left.\mathrm{H}_{2} \mathrm{O}\right)$. 
Supporting Information

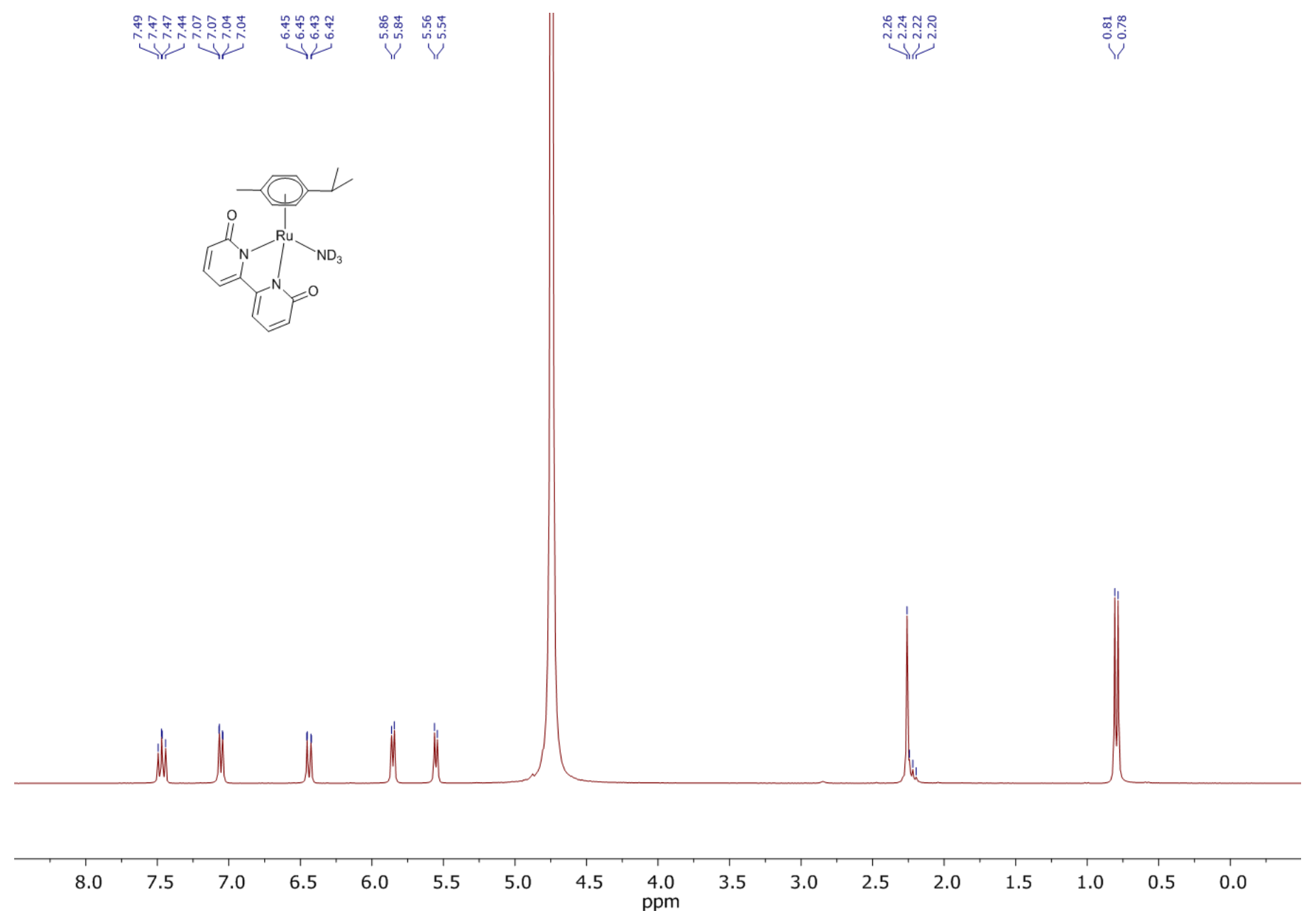

Figure S51. ${ }^{1} \mathrm{H}$ NMR spectra (300 MHz). $3.89 \mathrm{mg}$ of $[\mathrm{Ru}(\mathrm{p}-\mathrm{Cym})(\mathrm{dhbp}) \mathrm{Cl}] \mathrm{Cl}(\mathbf{1})$ in $0.5 \mathrm{~mL}$ of $\mathrm{D}_{2} \mathrm{O}$, after addition of $20 \mu \mathrm{L}$ of $\mathrm{NH}_{3}\left(20 \% \mathrm{v} / \mathrm{v}\right.$ in $\left.\mathrm{H}_{2} \mathrm{O}\right)$. 


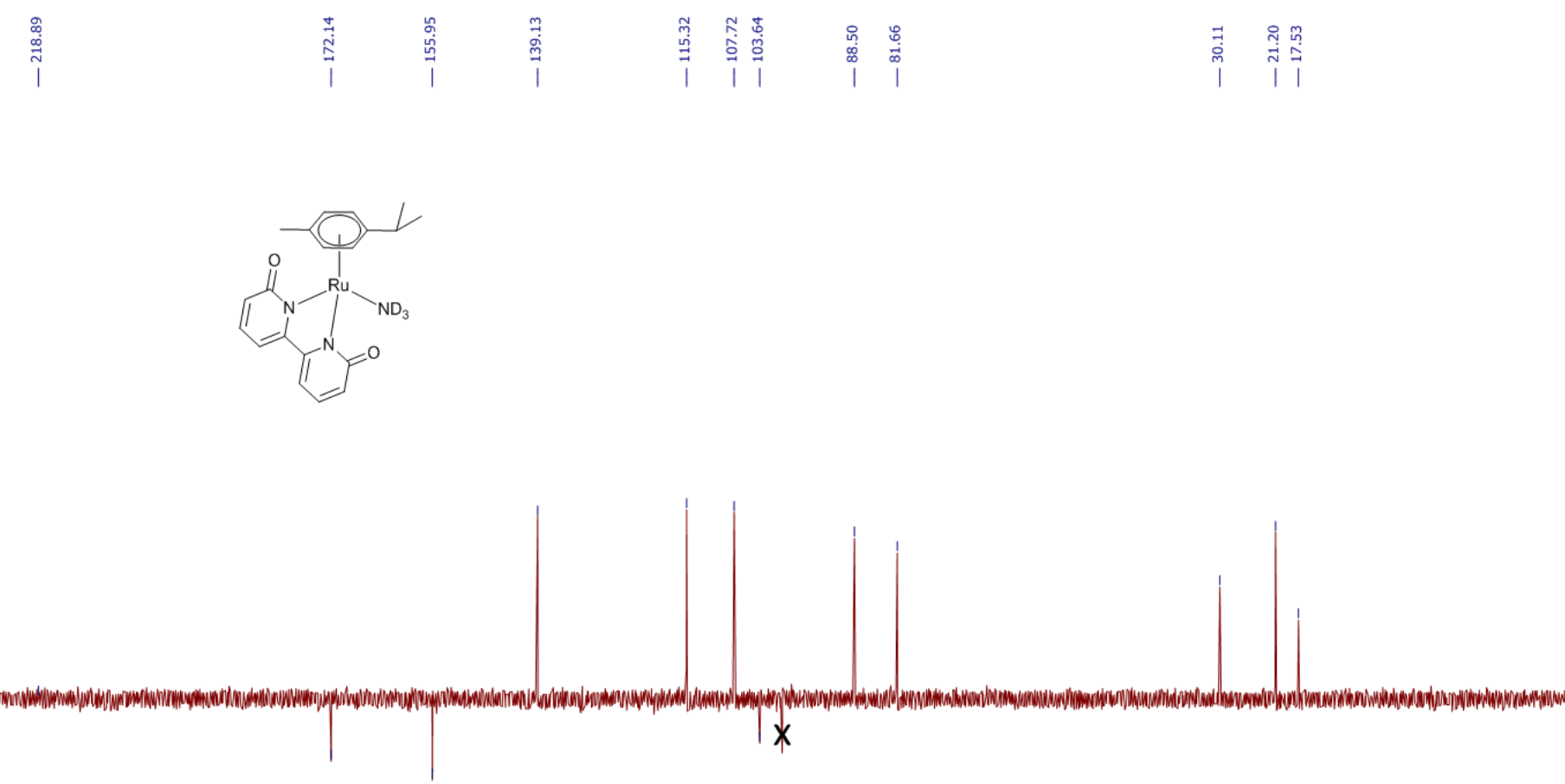

$\begin{array}{lllllllllllllllllllllllll}220 & 210 & 200 & 190 & 180 & 170 & 160 & 150 & 140 & 130 & 120 & 110 & 100 & 90 & 80 & 70 & 60 & 50 & 40 & 30 & 20 & 10 & 0 & -10 & -20\end{array}$

Figure S52. ${ }^{13} \mathrm{C}(\mathrm{APT}) \mathrm{NMR}$ spectra $(75 \mathrm{MHz}) .3 .89 \mathrm{mg}$ of $[\mathrm{Ru}(\mathrm{p}-\mathrm{Cym})(\mathrm{dhbp}) \mathrm{Cl}] \mathrm{Cl}(\mathbf{1})$ in $0.5 \mathrm{~mL}$ of $\mathrm{D}_{2} \mathrm{O}$, after addition of $20 \mu \mathrm{L}$ of $\mathrm{NH}_{3}\left(20 \% \mathrm{v} / \mathrm{v}\right.$ in $\left.\mathrm{H}_{2} \mathrm{O}\right)$. One of the quaternary carbons of $\mathrm{p}$-Cymene is hidden in the background noise of the spectra. Peak at $100 \mathrm{ppm}$ is an artifact (center of the acquisition window). 


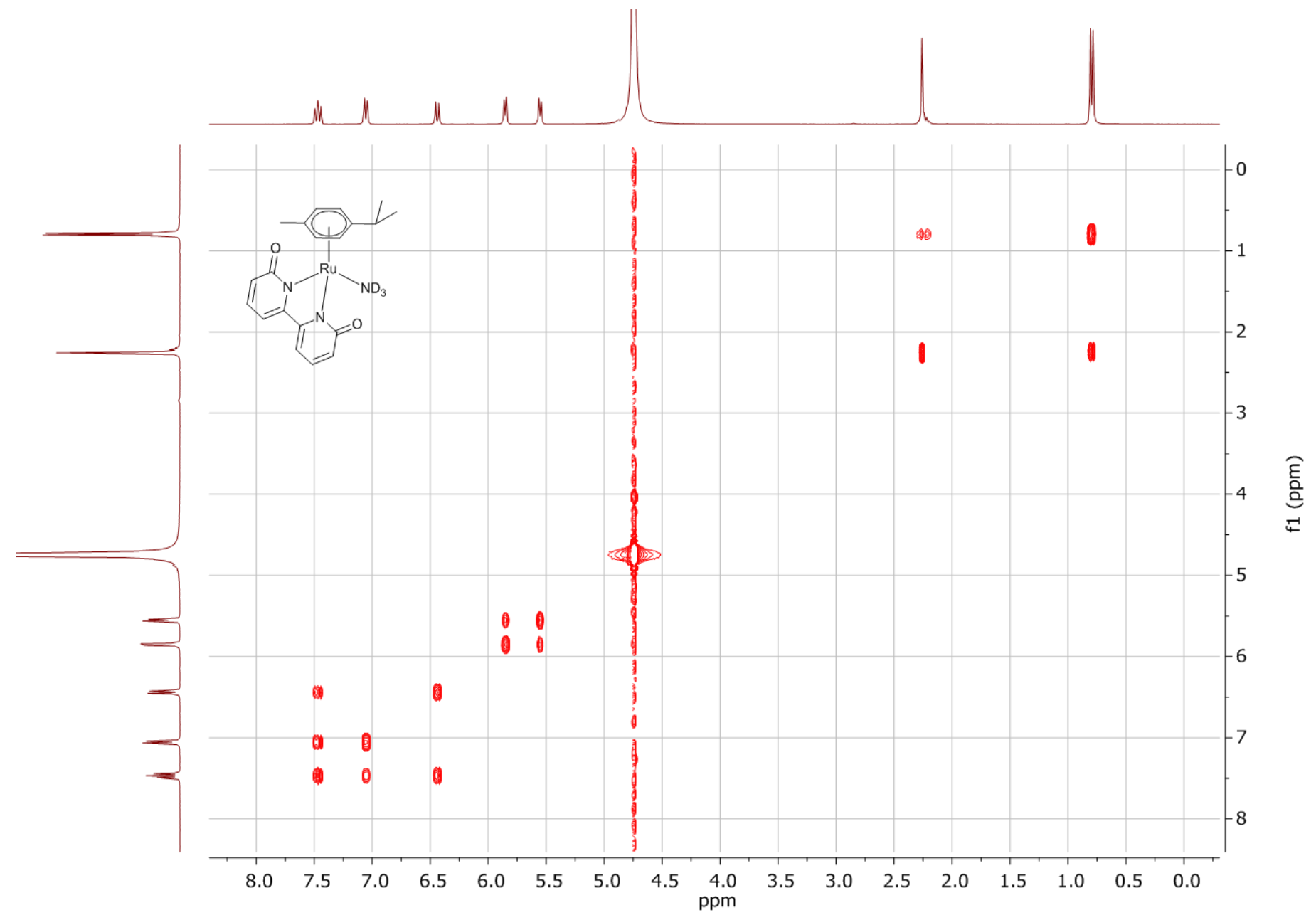

Figure S53. COSY NMR spectra (300 MHz). $3.89 \mathrm{mg}$ of $[\mathrm{Ru}(\mathrm{p}-\mathrm{Cym})(\mathrm{dhbp}) \mathrm{Cl}] \mathrm{Cl}(\mathbf{1})$ in $0.5 \mathrm{~mL}$ of $\mathrm{D}_{2} \mathrm{O}$, after addition of $20 \mu \mathrm{L}$ of $\mathrm{NH}_{3}\left(20 \% \mathrm{v} / \mathrm{v}\right.$ in $\left.\mathrm{H}_{2} \mathrm{O}\right)$. 


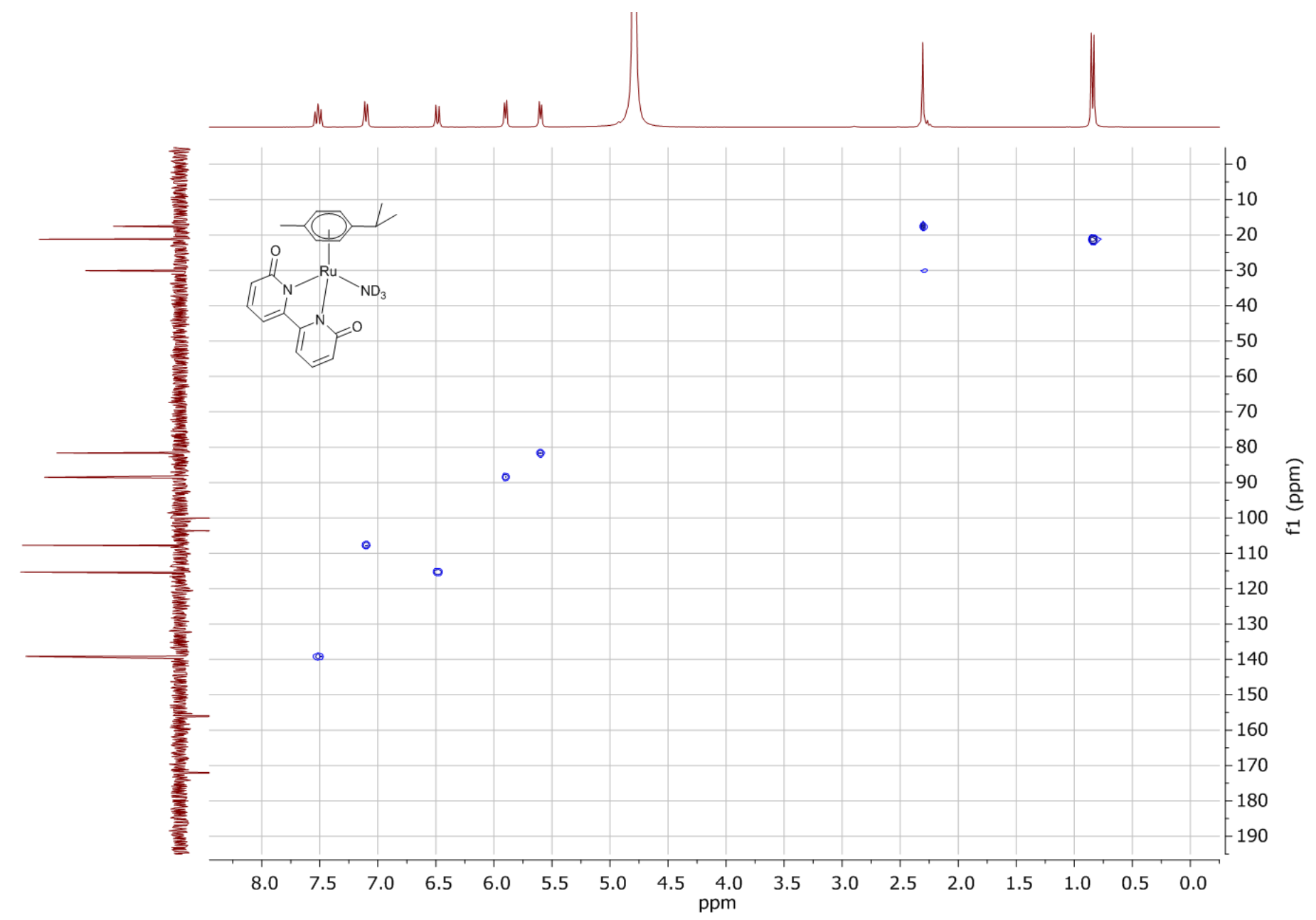

Figure S54. HSQC NMR spectra (300 MHz). $3.89 \mathrm{mg}$ of $[\mathrm{Ru}(\mathrm{p}-\mathrm{Cym})(\mathrm{dhbp}) \mathrm{Cl}] \mathrm{Cl}(\mathbf{1})$ in $0.5 \mathrm{~mL}$ of $\mathrm{D}_{2} \mathrm{O}$, after addition of $20 \mu \mathrm{L}$ of $\mathrm{NH}_{3}\left(20 \% \mathrm{v} / \mathrm{v}\right.$ in $\left.\mathrm{H}_{2} \mathrm{O}\right)$. 
In situ characterization of $[\mathrm{Ru}(\mathrm{p}-\mathrm{Cym})(\mathrm{dhbp}-2 \mathrm{H})(\mathrm{OD})]^{-}\left(0.001 \mathrm{M} \mathrm{NaOD} / \mathrm{D}_{2} \mathrm{O}\right)$ :

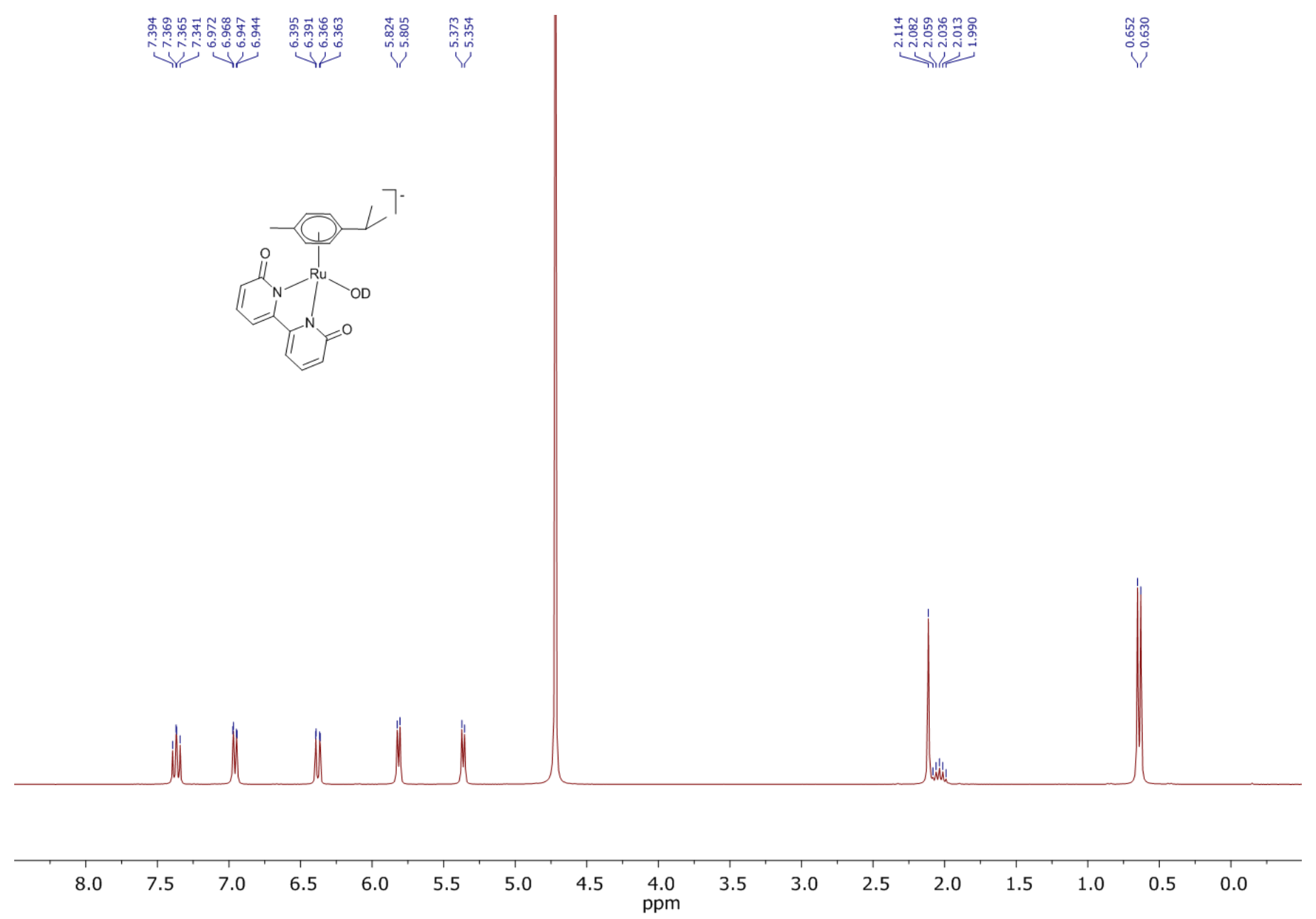

Figure S55. ${ }^{1} \mathrm{H}$ NMR spectra (300 MHz). $4.03 \mathrm{mg}$ of $[\mathrm{Ru}(\mathrm{p}-\mathrm{Cym})(\mathrm{dhbp}) \mathrm{Cl}] \mathrm{Cl}(\mathbf{1})$ in $0.5 \mathrm{~mL}$ of $0.001 \mathrm{M} \mathrm{NaOD}$ in $\mathrm{D}_{2} \mathrm{O}$. 


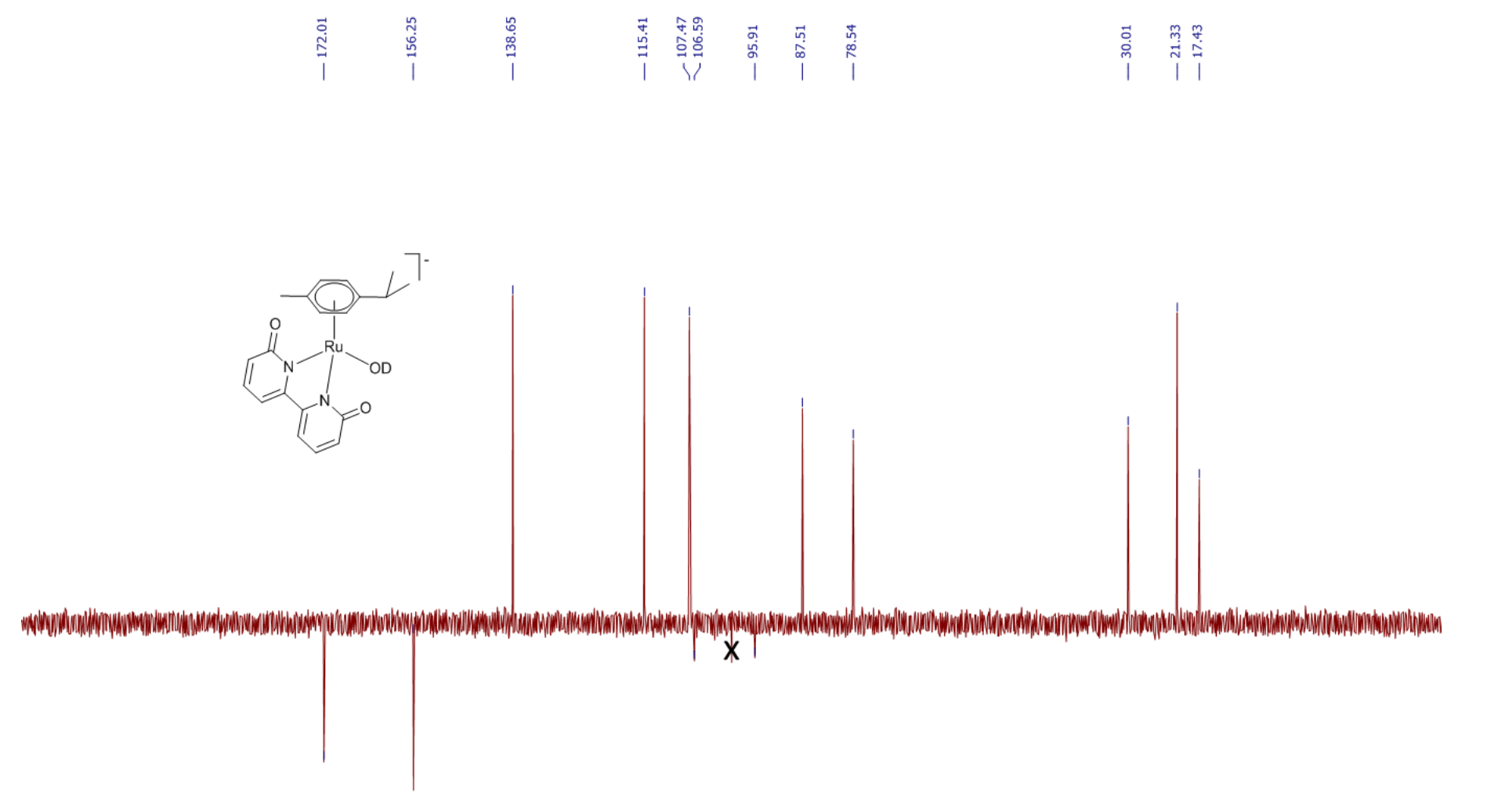

$\begin{array}{llllllllllllllllllllllllllllllll}220 & 210 & 200 & 190 & 180 & 170 & 160 & 150 & 140 & 130 & 120 & 110 & 100 & 90 & 80 & 70 & 60 & 50 & 40 & 30 & 20 & 10 & 0 & -10 & -20\end{array}$

Figure S56. ${ }^{13} \mathrm{C}(\mathrm{APT}) \mathrm{NMR}$ spectra $(75 \mathrm{MHz}) .4 .03 \mathrm{mg}$ of $[\mathrm{Ru}(\mathrm{p}-\mathrm{Cym})(\mathrm{dhbp}) \mathrm{Cl}] \mathrm{Cl}(\mathbf{1})$ in $0.5 \mathrm{~mL}$ of $0.001 \mathrm{M} \mathrm{NaOD}$ in $\mathrm{D}_{2} \mathrm{O}$. Peak at $100 \mathrm{ppm}$ is an artifact (center of the acquisition window). 


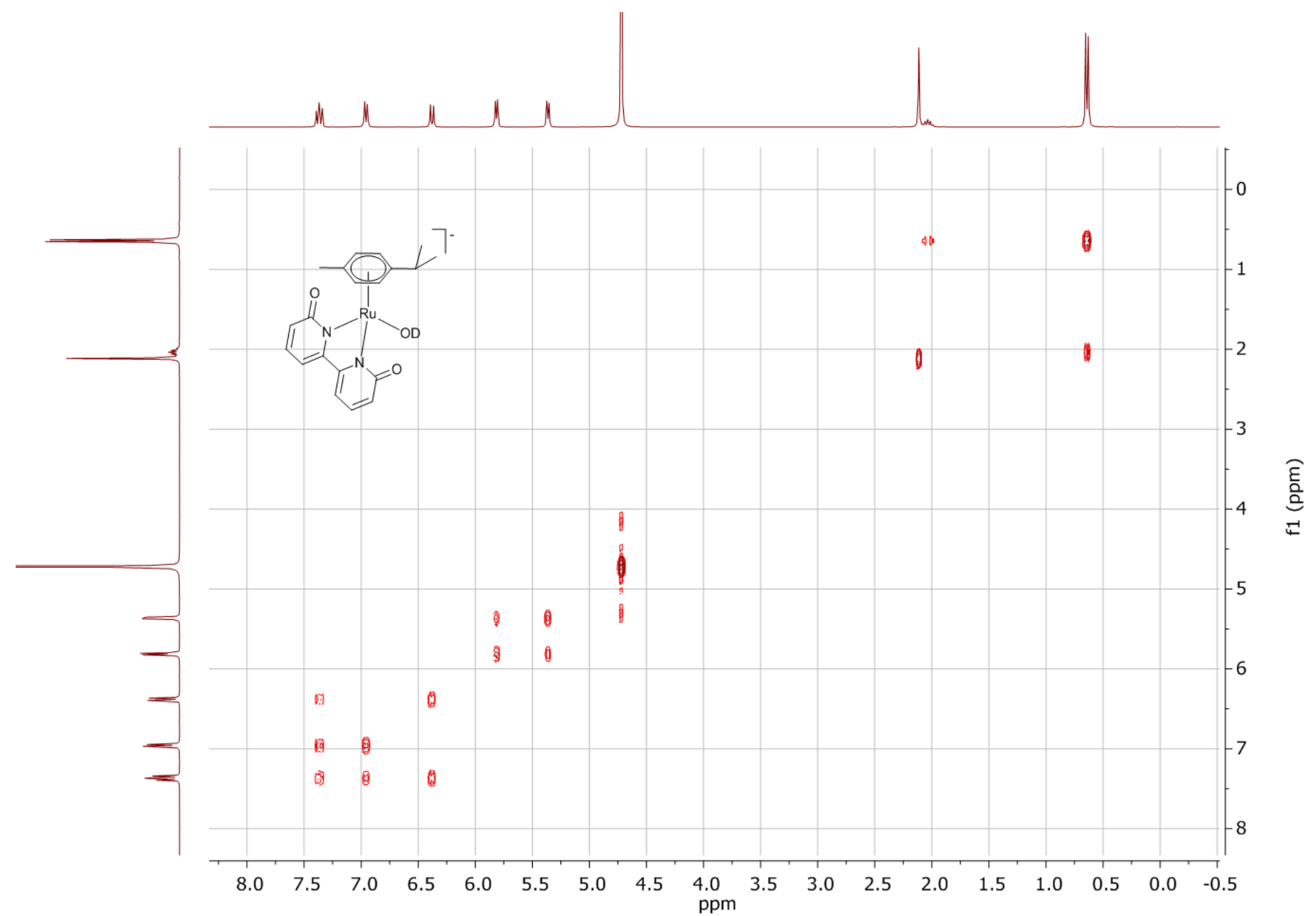

Figure S57. COSY NMR spectra (300 MHz). $4.03 \mathrm{mg}$ of $[\mathrm{Ru}(\mathrm{p}-\mathrm{Cym})(\mathrm{dhbp}) \mathrm{Cl}] \mathrm{Cl}(\mathbf{1})$ in $0.5 \mathrm{~mL}$ of $0.001 \mathrm{M} \mathrm{NaOD}$ in $\mathrm{D}_{2} \mathrm{O}$. 


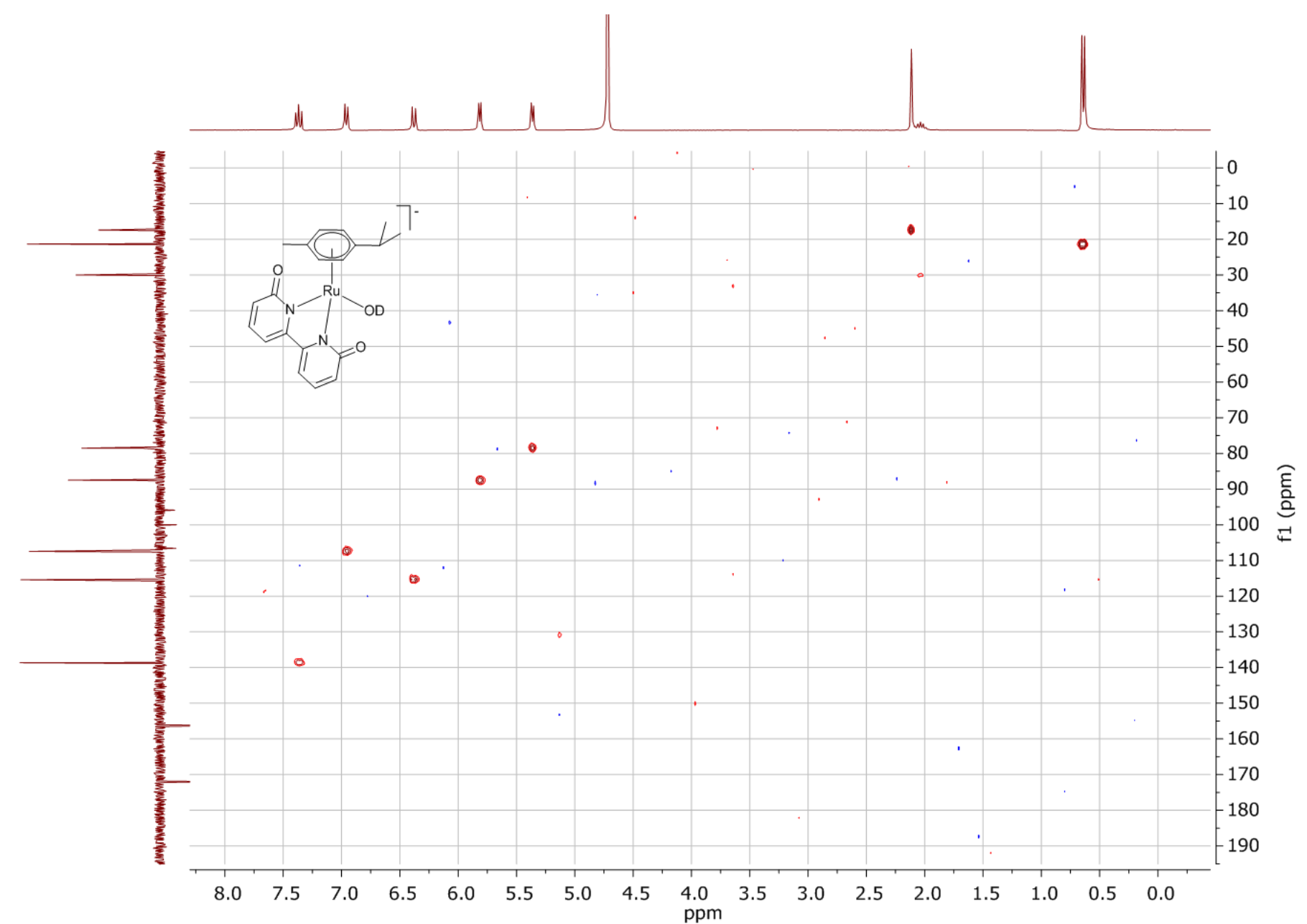

Figure S58. HSQC NMR spectra (300 MHz). $4.03 \mathrm{mg}$ of $[\mathrm{Ru}(\mathrm{p}-\mathrm{Cym})(\mathrm{dhbp}) \mathrm{Cl}] \mathrm{Cl}(\mathbf{1})$ in $0.5 \mathrm{~mL}$ of $0.001 \mathrm{M} \mathrm{NaOD}$ in $\mathrm{D}_{2} \mathrm{O}$. 
Scheme S2. Reactivity scheme used for the in situ characterizations in $\mathrm{CD}_{3} \mathrm{OD}$ :

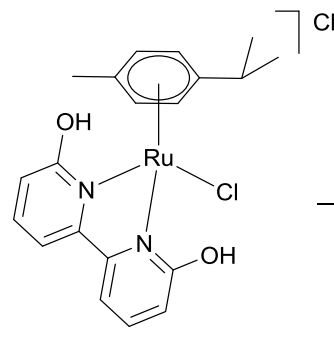

1

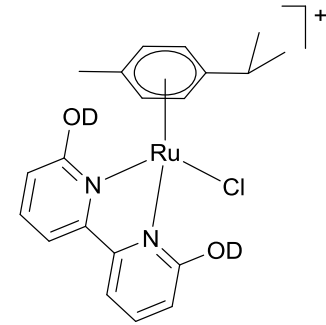

$\left[R u(p-C y m)\left(\right.\right.$ dhbp)Cl] ${ }^{+}$

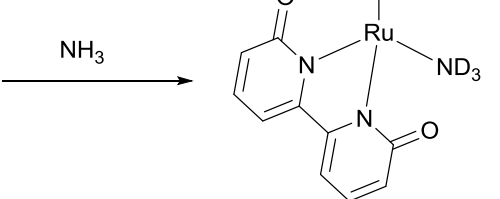

$\left[\mathrm{Ru}(\mathrm{p}-\mathrm{Cym})(\mathrm{dhbp}-2 \mathrm{H})\left(\mathrm{D}_{2} \mathrm{O}\right)\right]$ 
Characterization of $[\mathrm{Ru}(\mathrm{p}-\mathrm{Cym})(\mathrm{dhbp}) \mathrm{Cl}] \mathrm{Cl}(1)$ in $\mathrm{CD}_{3} \mathrm{OD}$ :

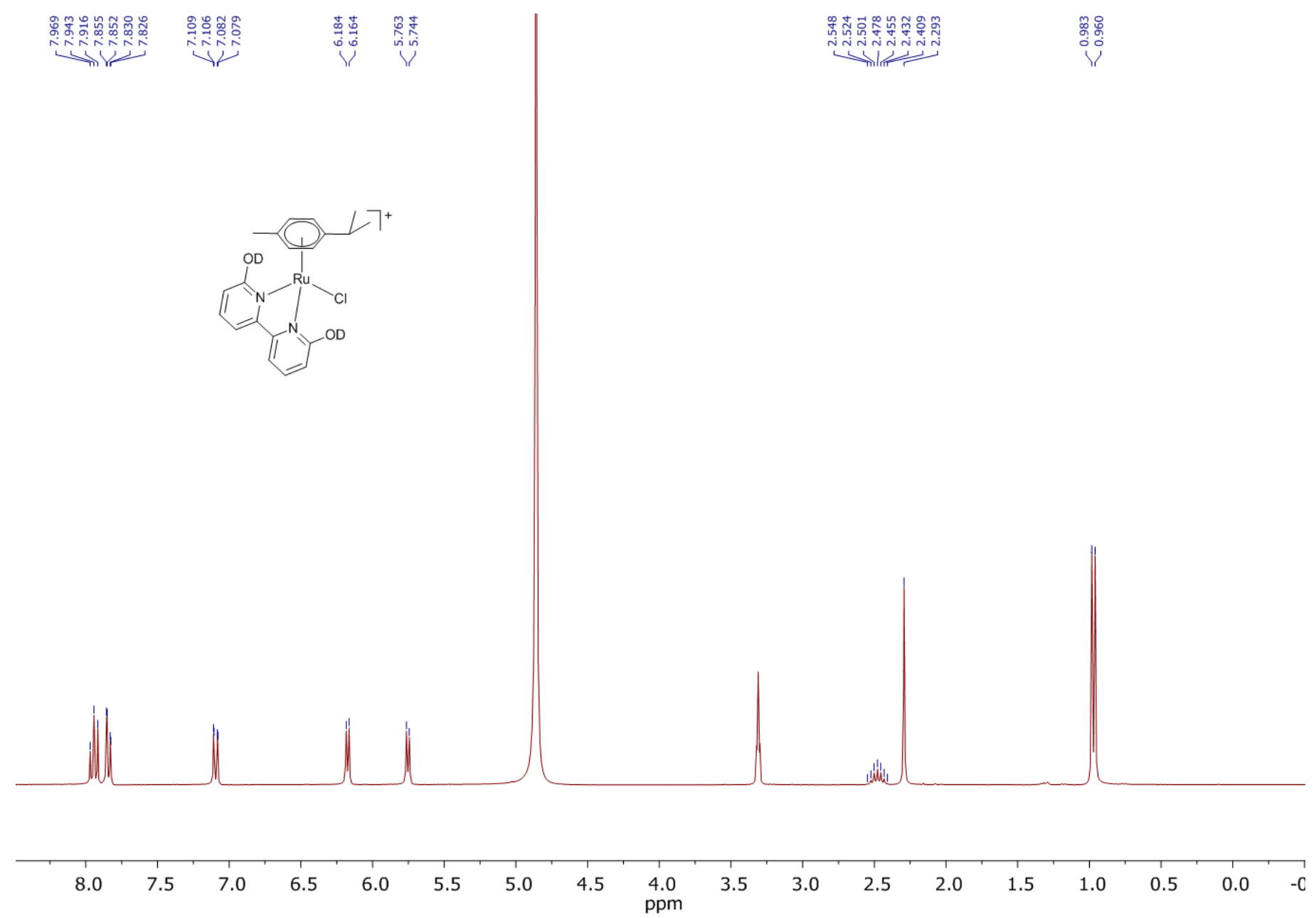

Figure $559{ }^{1} \mathrm{H}$ NMR spectra (300 MHz). $3.85 \mathrm{mg}$ of $[\mathrm{Ru}(\mathrm{p}-\mathrm{Cym})(\mathrm{dhbp}) \mathrm{Cl}] \mathrm{Cl}(\mathbf{1})$ in $0.5 \mathrm{~mL}$ of $\mathrm{CD}_{3} \mathrm{OD}$. 


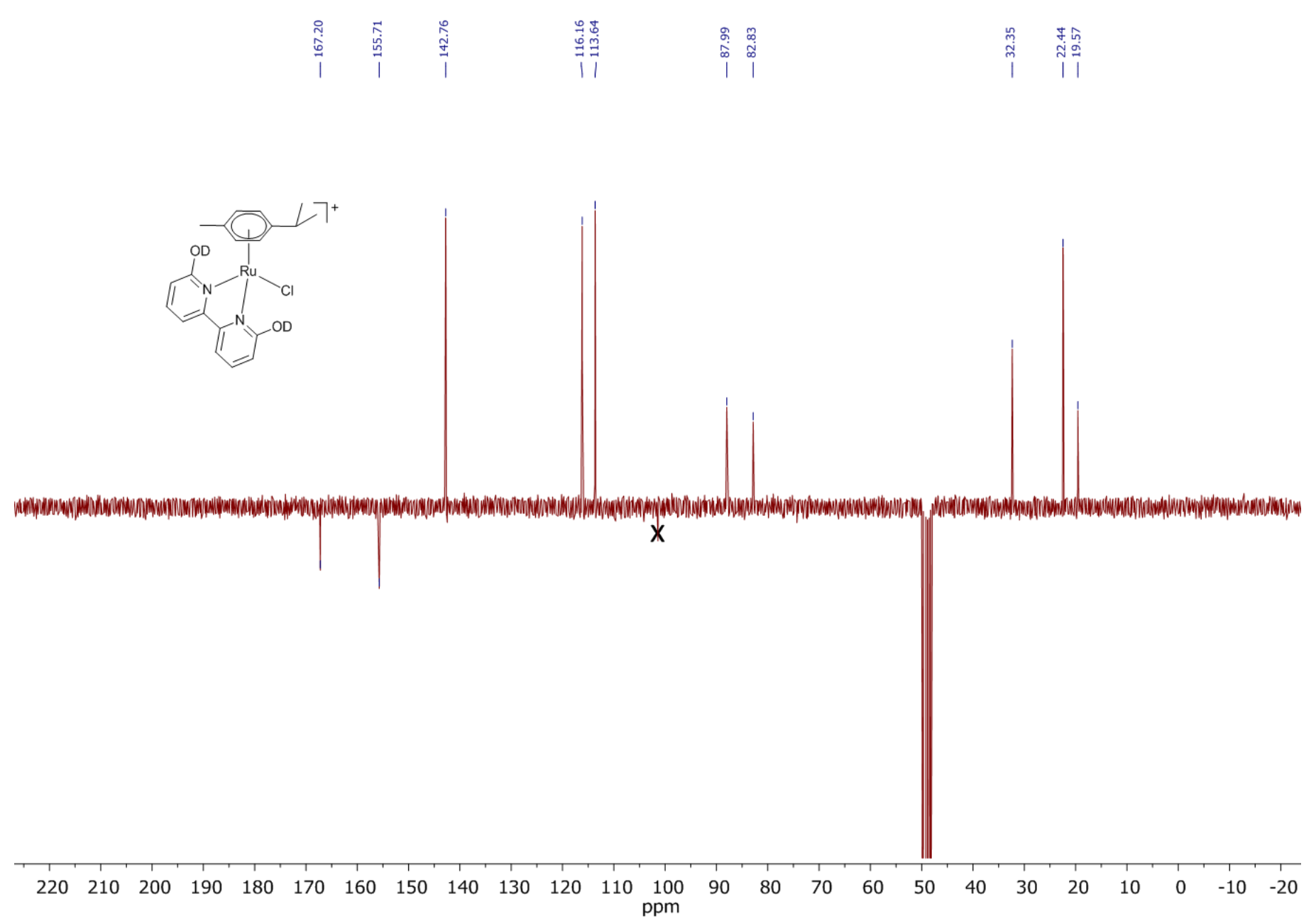

Figure S60. ${ }^{13} \mathrm{C}(\mathrm{APT}) \mathrm{NMR}$ spectra $(75 \mathrm{MHz}) .3 .85 \mathrm{mg}$ of $[\mathrm{Ru}(\mathrm{p}-\mathrm{Cym})(\mathrm{dhbp}) \mathrm{Cl}] \mathrm{Cl}(\mathbf{1})$ in $0.5 \mathrm{~mL}$ of $\mathrm{CD}_{3} \mathrm{OD}$. Quaternary carbons of $\mathrm{p}-\mathrm{Cymene}$ are hidden in the background noise of the spectra. Peak at 100 ppm is an artifact (center of the acquisition window). 


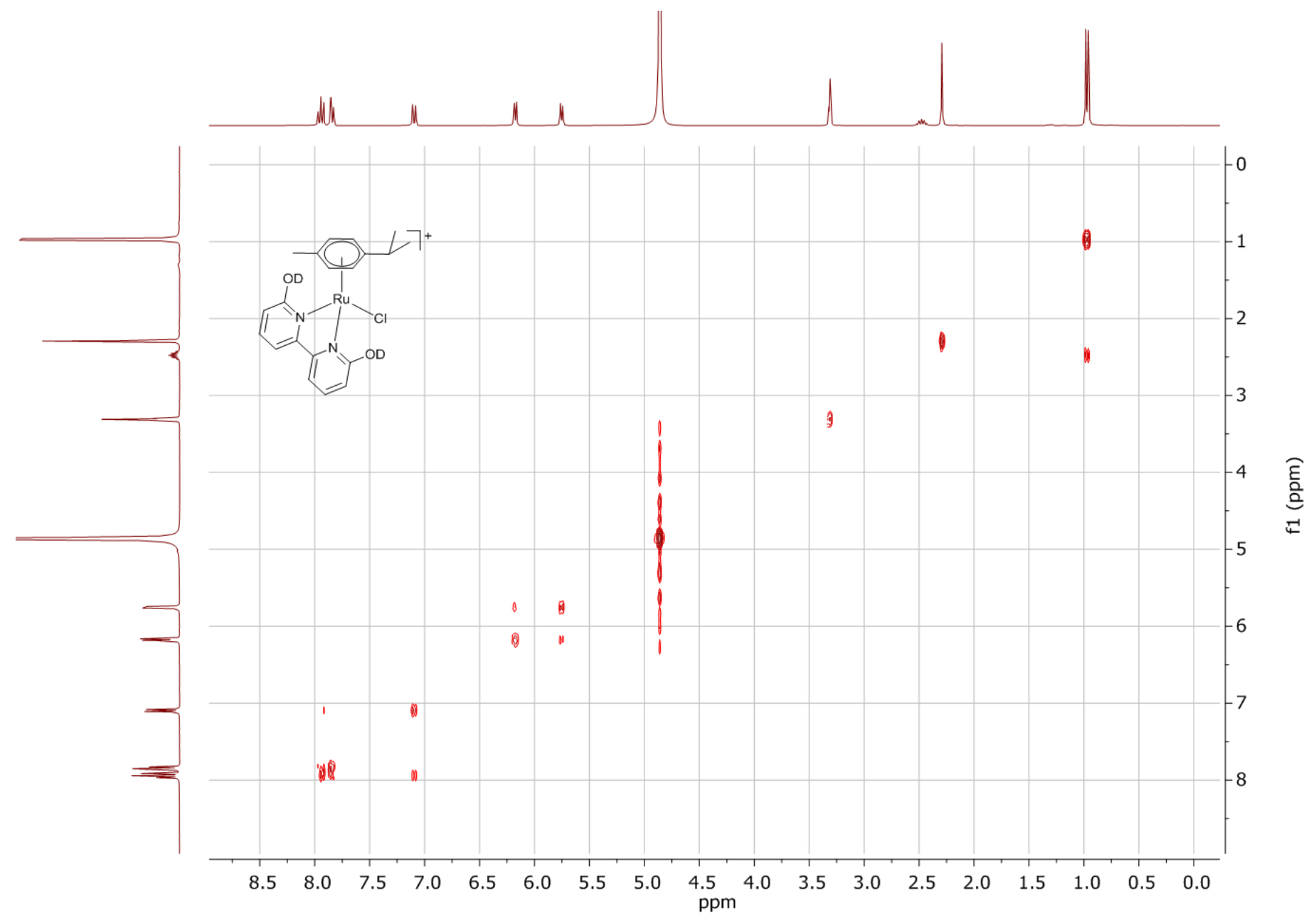

Figure S61. COSY NMR spectra (300 MHz). $3.85 \mathrm{mg}$ of $[\mathrm{Ru}(\mathrm{p}-\mathrm{Cym})(\mathrm{dhbp}) \mathrm{Cl}] \mathrm{Cl}(\mathbf{1})$ in $0.5 \mathrm{~mL}$ of $\mathrm{CD}_{3} \mathrm{OD}$. 


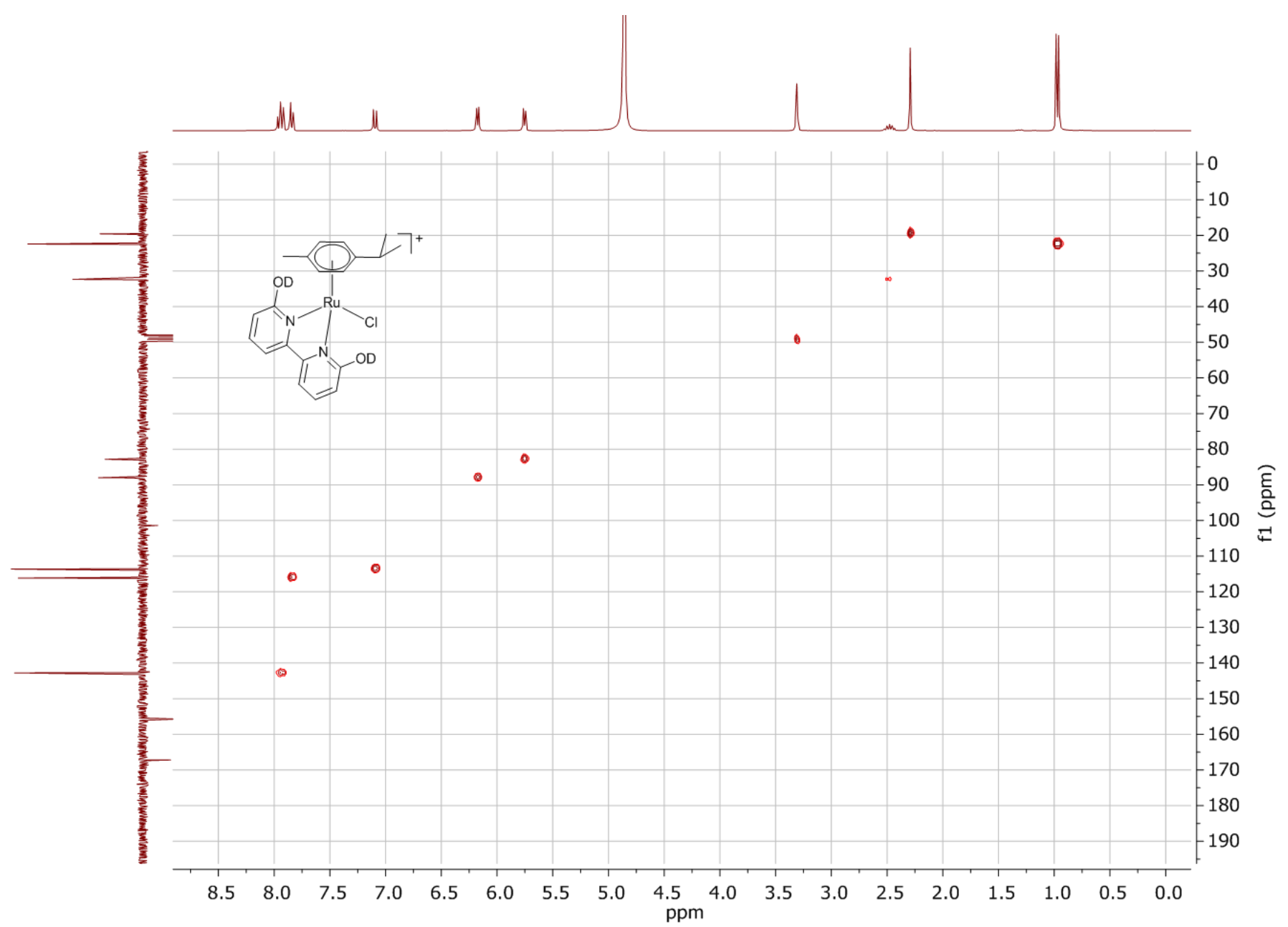

Figure S62. HSQC NMR spectra (300 MHz). $3.85 \mathrm{mg}$ of [Ru(p-Cym)(dhbp)Cl]Cl (1) in $0.5 \mathrm{~mL}$ of $\mathrm{CD}_{3} \mathrm{OD}$. 
In situ formation and characterization of $\left[\mathrm{Ru}(\mathrm{p}-\mathrm{Cym})(\mathrm{dhbp}-2 \mathrm{H})\left(\mathrm{ND}_{3}\right)\right]\left(\mathrm{CD}_{3} \mathrm{OD}\right)$ :

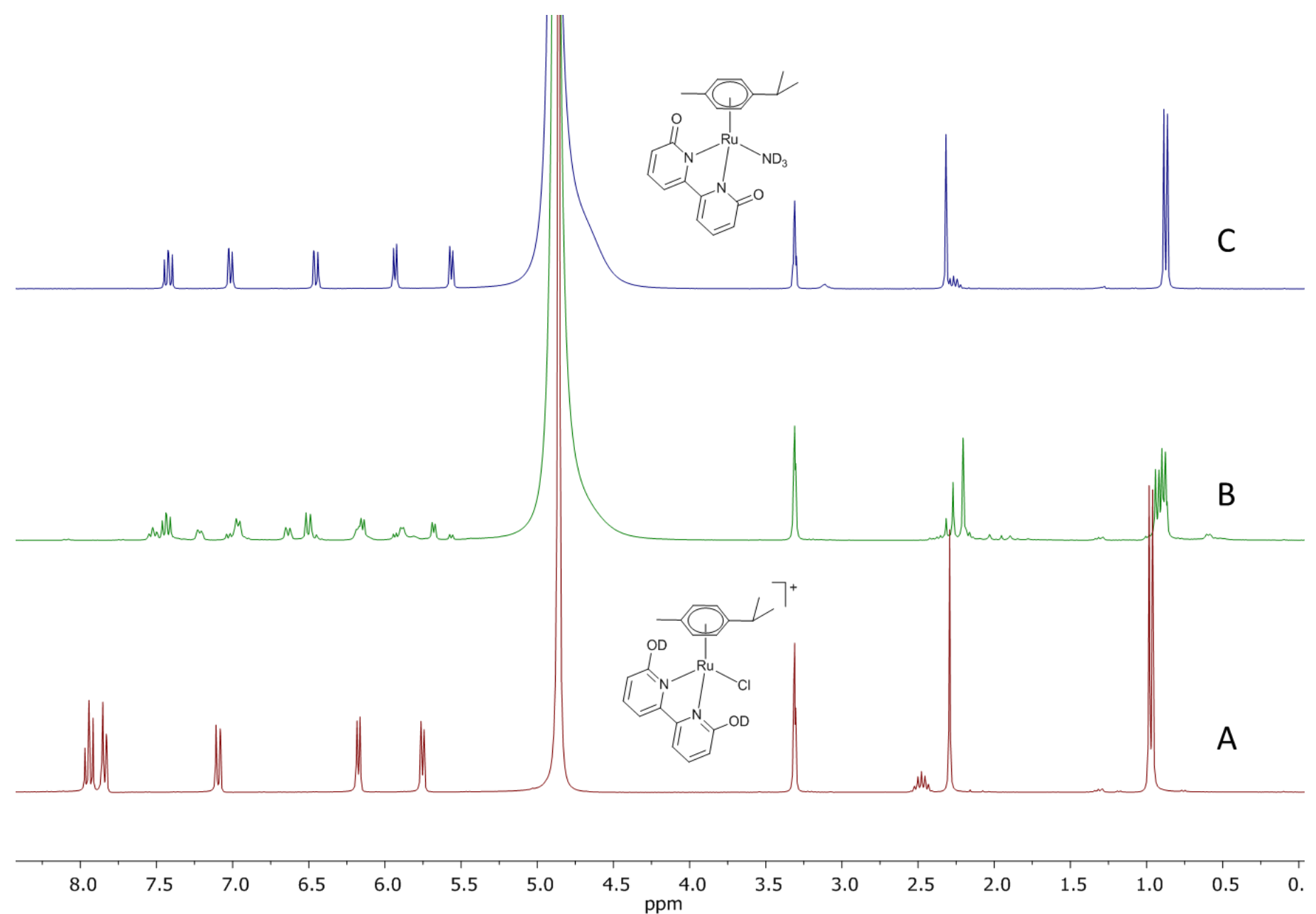

Figure S63. ${ }^{1} \mathrm{H}$ NMR spectra (300 MHz). $3.85 \mathrm{mg}$ of $[\mathrm{Ru}(\mathrm{p}-\mathrm{Cym})(\mathrm{dhbp}) \mathrm{Cl}] \mathrm{Cl}(\mathbf{1})$ and in $0.5 \mathrm{~mL}$ of $\mathrm{CD}_{3} \mathrm{OD}$. B: After addition of $10 \mu \mathrm{L}$ of $\mathrm{NH}_{3}\left(20 \% \mathrm{v} / \mathrm{v}\right.$ in $\left.\mathrm{H}_{2} \mathrm{O}\right)$. B: After addition of $10 \mu \mathrm{L}$ more of $\mathrm{NH}_{3}\left(20 \% \mathrm{v} / \mathrm{v}\right.$ in $\left.\mathrm{H}_{2} \mathrm{O}\right)$. 


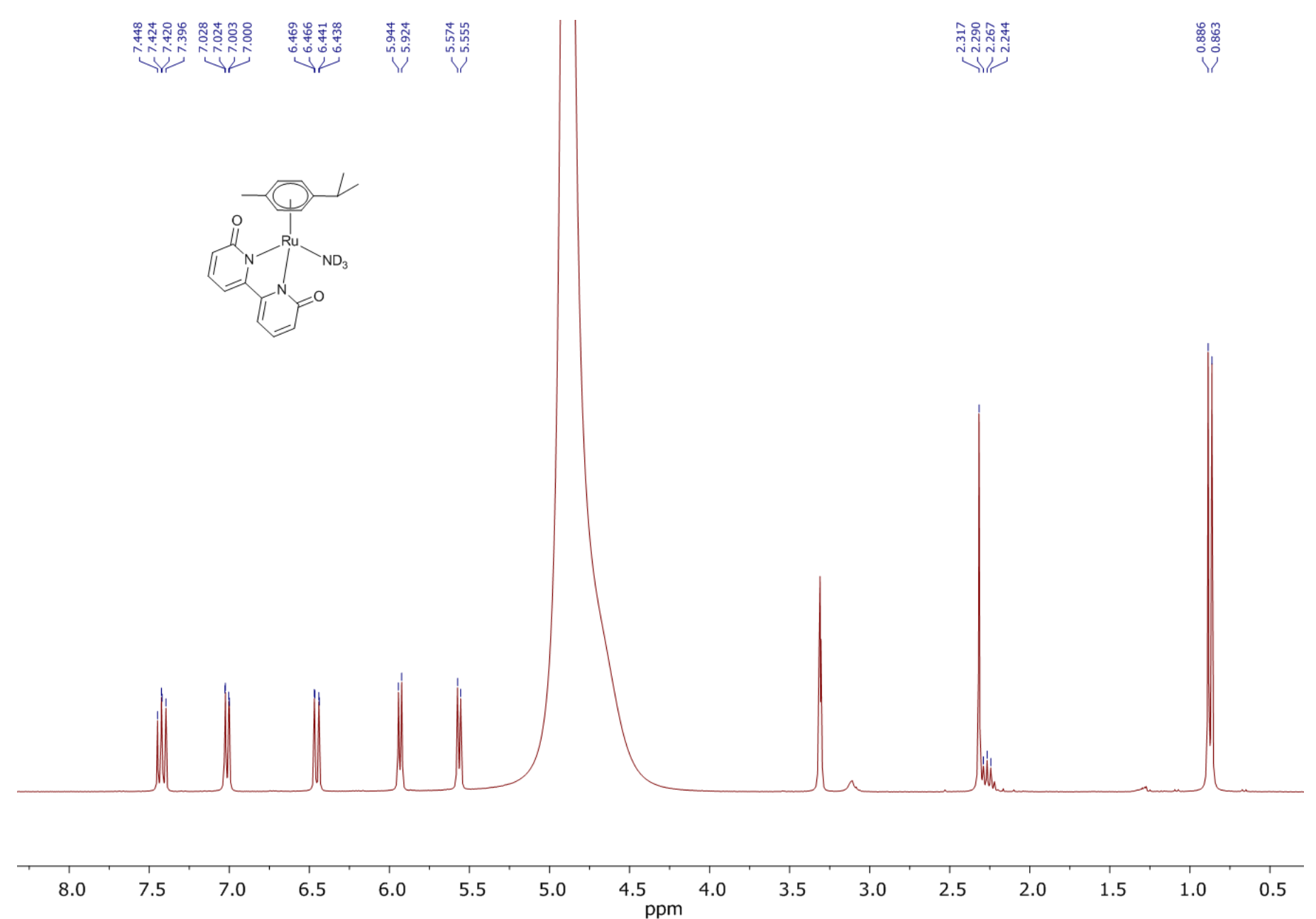

Figure S64. ${ }^{1} \mathrm{H}$ NMR spectra (300 MHz). $3.85 \mathrm{mg}$ of $[\mathrm{Ru}(\mathrm{p}-\mathrm{Cym})(\mathrm{dhbp}) \mathrm{Cl}] \mathrm{Cl}(\mathbf{1})$ in $0.5 \mathrm{~mL}$ of $\mathrm{CD}_{3} \mathrm{OD}$, after addition of $20 \mu \mathrm{L}$ of $\mathrm{NH}_{3}\left(20 \% \mathrm{v} / \mathrm{v}\right.$ in $\left.\mathrm{H}_{2} \mathrm{O}\right)$. 


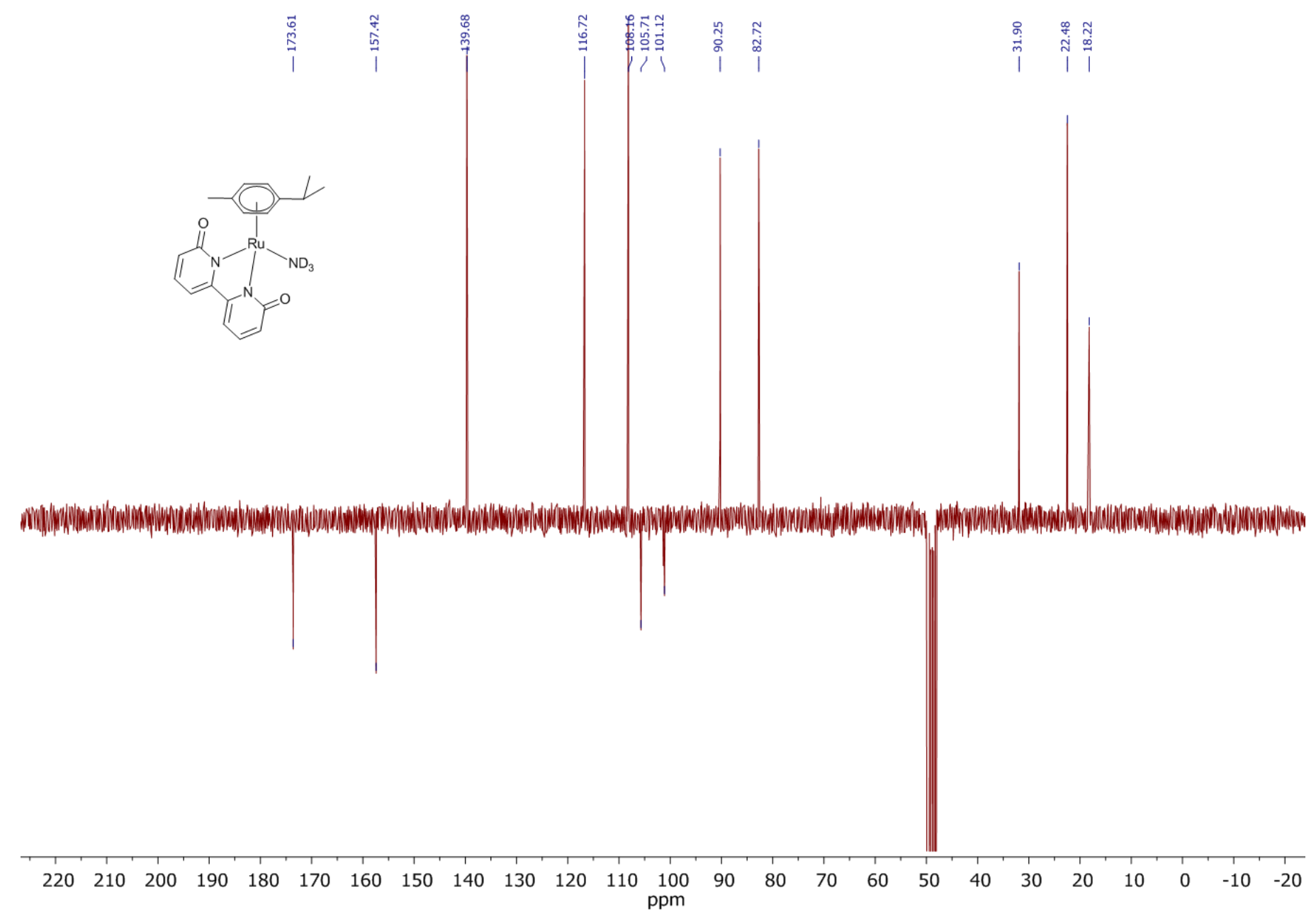

Figure S65. ${ }^{13} \mathrm{C}(\mathrm{APT}) \mathrm{NMR}$ spectra $(75 \mathrm{MHz}) .3 .85 \mathrm{mg}$ of $[\mathrm{Ru}(\mathrm{p}-\mathrm{Cym})(\mathrm{dhbp}) \mathrm{Cl}] \mathrm{Cl}(\mathbf{1})$ in $0.5 \mathrm{~mL}$ of $\mathrm{CD}_{3} \mathrm{OD}$, after addition of $20 \mu \mathrm{L}$ of $\mathrm{NH}_{3}\left(20 \% \mathrm{v} / \mathrm{v}\right.$ in $\left.\mathrm{H}_{2} \mathrm{O}\right)$. One of the quaternary carbons of $\mathrm{p}$-Cymene is hidden in the background noise of the spectra. Peak at $100 \mathrm{ppm}$ is an artifact (center of the acquisition window). 


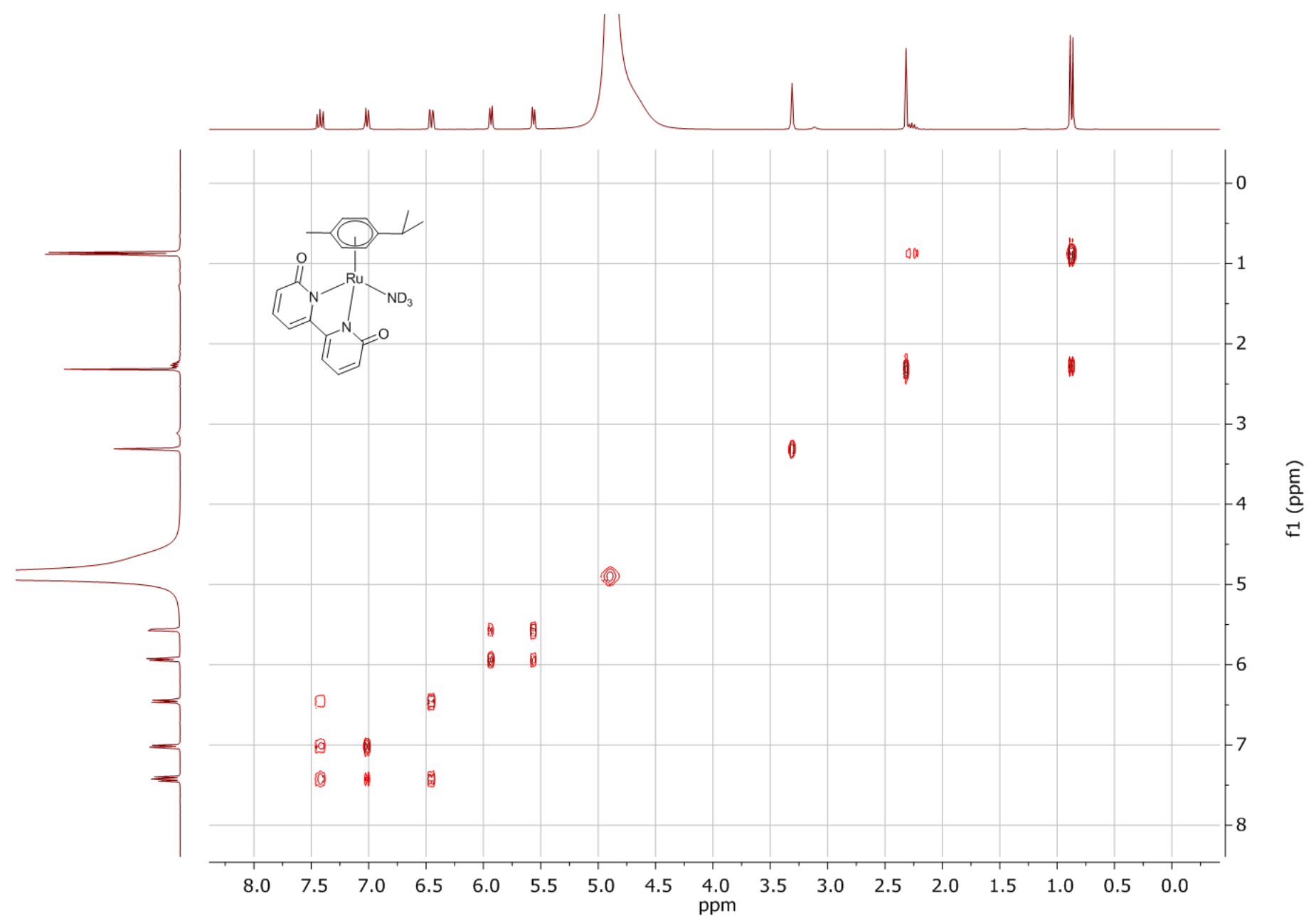

Figure S66. COSY NMR spectra (300 MHz). $3.85 \mathrm{mg}$ of $[\mathrm{Ru}(\mathrm{p}-\mathrm{Cym})(\mathrm{dhbp}) \mathrm{Cl}] \mathrm{Cl}(\mathbf{1})$ in $0.5 \mathrm{~mL}$ of $\mathrm{CD}_{3} \mathrm{OD}$, after addition of $20 \mu \mathrm{L}$ of $\mathrm{NH}_{3}\left(20 \% \mathrm{v} / \mathrm{v}\right.$ in $\left.\mathrm{H}_{2} \mathrm{O}\right)$. 


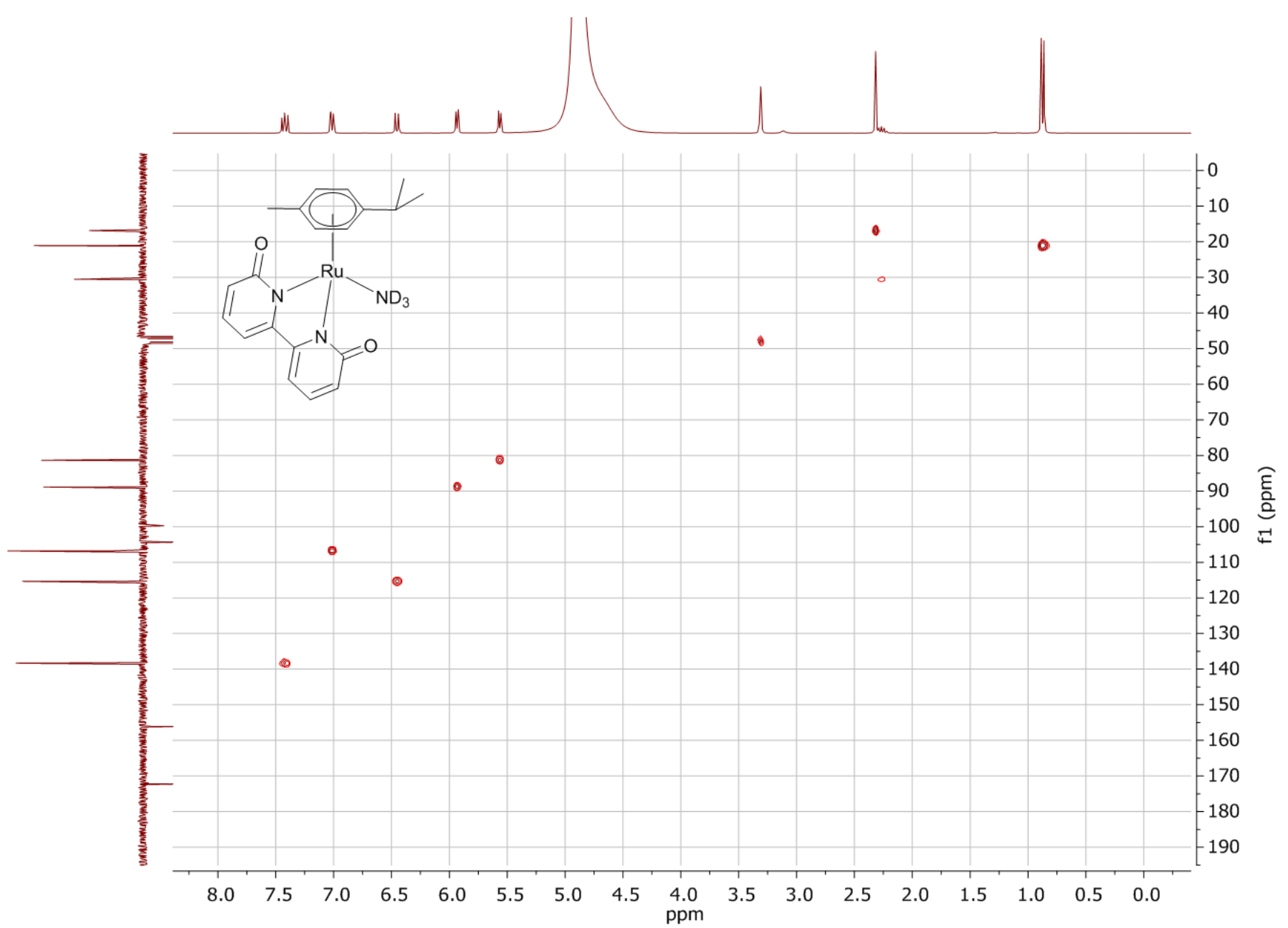

Figure S67. HSQC NMR spectra (300 MHz). $3.85 \mathrm{mg}$ of $[\mathrm{Ru}(\mathrm{p}-\mathrm{Cym})(\mathrm{dhbp}) \mathrm{Cl}] \mathrm{Cl}(\mathbf{1})$ in $0.5 \mathrm{~mL}$ of $\mathrm{CD}_{3} \mathrm{OD}$, after addition of $20 \mu \mathrm{L}$ of $\mathrm{NH}_{3}\left(20 \% \mathrm{v} / \mathrm{v}\right.$ in $\left.\mathrm{H}_{2} \mathrm{O}\right)$. 
In situ formation and characterization of $[\mathrm{Ru}(\mathrm{p}-\mathrm{Cym})(\mathrm{dhbp}-2 \mathrm{H}) \mathrm{H}]^{-}\left(0.0001 \mathrm{M} \mathrm{NaOD} / \mathrm{D}_{2} \mathrm{O}\right)$ :

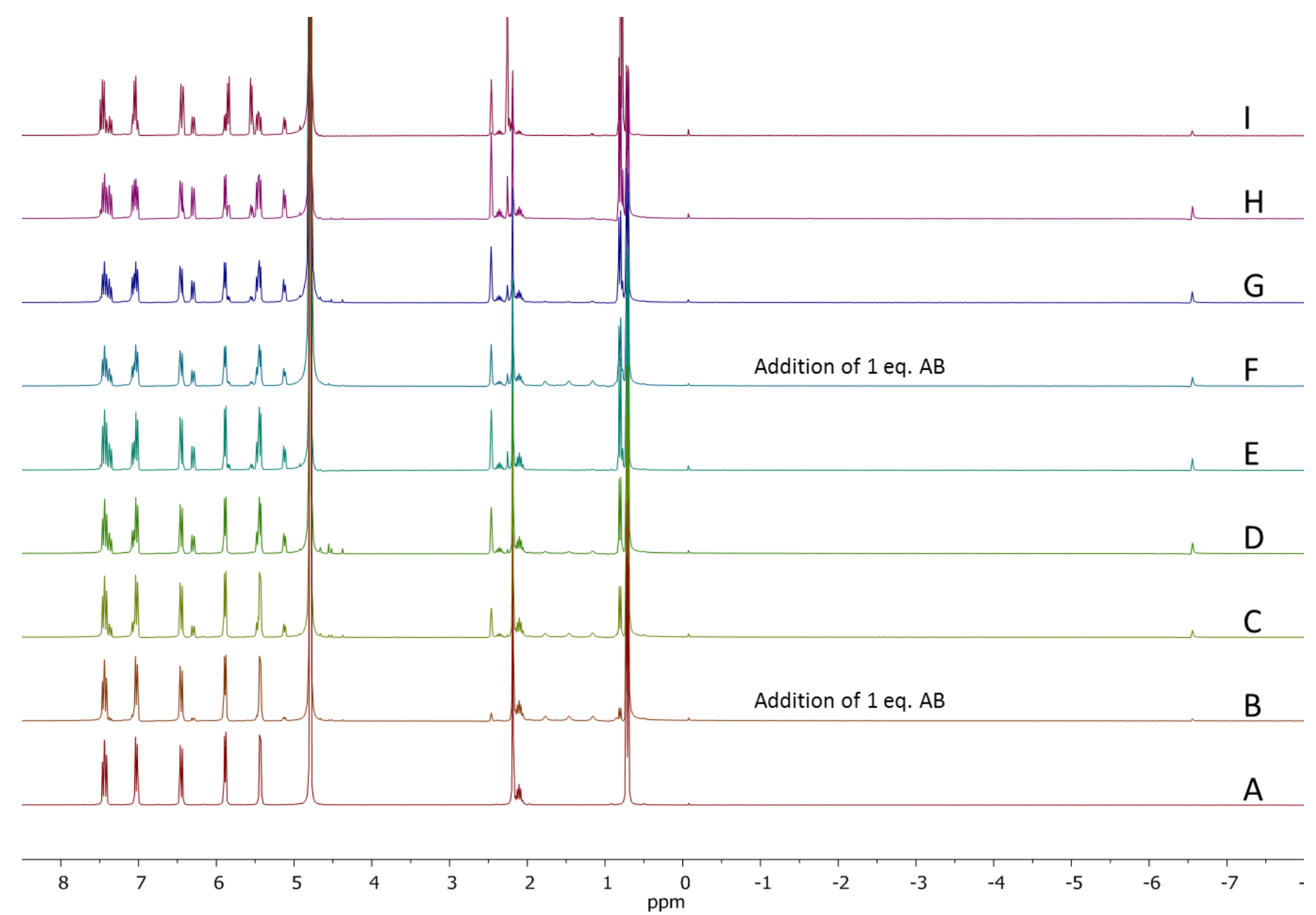

Figure S68. ${ }^{1} \mathrm{H}$ NMR spectra (300 MHz, 8.5 to $\left.-8.0 \mathrm{ppm}\right) \mathrm{A}: 4.03 \mathrm{mg}$ of $[\mathrm{Ru}(\mathrm{p}-\mathrm{Cym})(\mathrm{dhbp}) \mathrm{Cl}] \mathrm{Cl}(\mathbf{1})$ in $0.5 \mathrm{~mL}$ of $0.001 \mathrm{M} \mathrm{NaOD}$ in $\mathrm{D}_{2} \mathrm{O}$. B-E: Time evolution after addition of $0.25 \mathrm{mg}$ of $A B(5 \mathrm{~min} ; 30 \mathrm{~min} ; 60 \mathrm{~min} ; 151 \mathrm{~min}$ ). F-I: Time evolution after addition of another $0.25 \mathrm{mg}$ of $A B(2 \mathrm{~min} ; 22 \mathrm{~min} ; 111 \mathrm{~min} ; 1340 \mathrm{~min}$ ). 


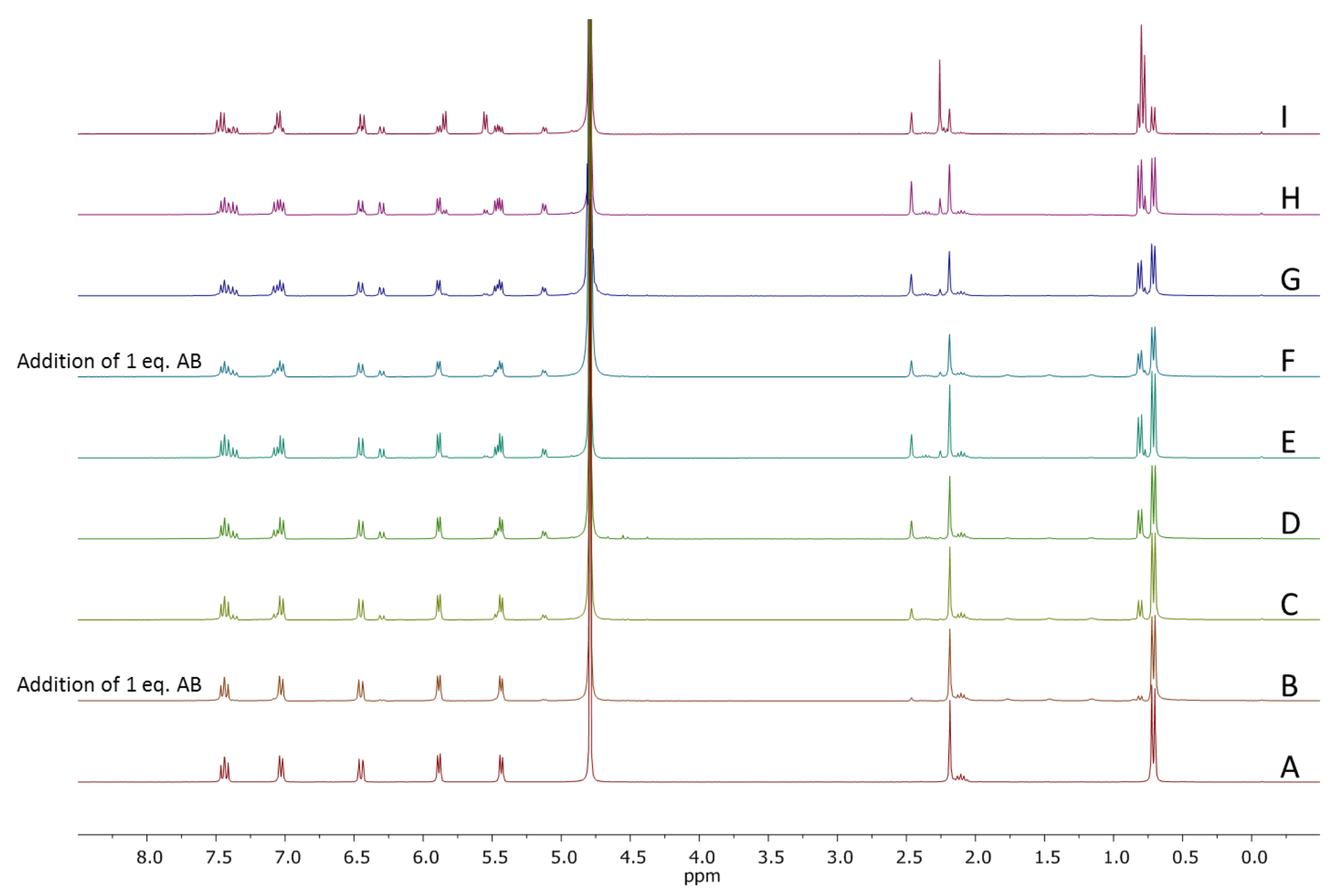

Figure S69. ${ }^{1} \mathrm{H}$ NMR spectra (300 MHz, 8.5 to $\left.-0.5 \mathrm{ppm}\right) \mathrm{A}: 4.03 \mathrm{mg}$ of $[\mathrm{Ru}(\mathrm{p}-\mathrm{Cym})(\mathrm{dhbp}) \mathrm{Cl}] \mathrm{Cl}(\mathbf{1})$ in $0.5 \mathrm{~mL}$ of $0.001 \mathrm{M} \mathrm{NaOD}$ in $\mathrm{D}_{2} \mathrm{O}$. B-E: Time evolution after addition of $0.25 \mathrm{mg}$ of $A B(5 \mathrm{~min} ; 30 \mathrm{~min} ; 60 \mathrm{~min} ; 151 \mathrm{~min}$ ). F-I: Time evolution after addition of another $0.25 \mathrm{mg}$ of $A B$ (2 min; $22 \mathrm{~min} ; 111 \mathrm{~min} ; 1340 \mathrm{~min}$ ). 


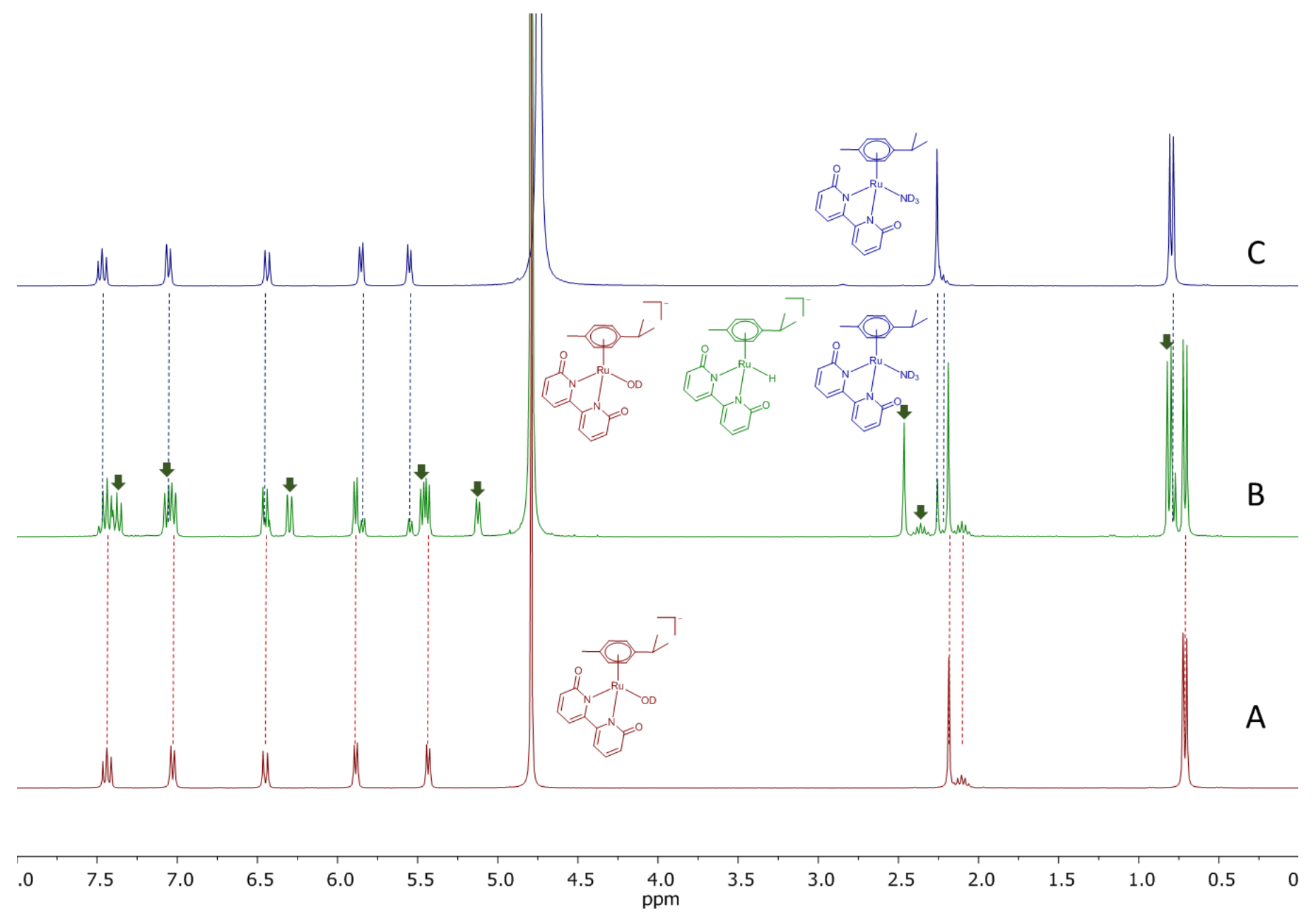

Figure S70. ${ }^{1} \mathrm{H}$ NMR spectra (300 MHz) of A: $4.03 \mathrm{mg}$ of $[\mathrm{Ru}(\mathrm{p}-\mathrm{Cym})(\mathrm{dhbp}) \mathrm{Cl}] \mathrm{Cl}(\mathbf{1})$ in $0.5 \mathrm{~mL}$ of $0.001 \mathrm{M} \mathrm{NaOD}$ in $\mathrm{D}_{2} \mathrm{O}$. B: $111 \mathrm{~min}$ after addition of 2 eq. of $\mathrm{AB}$. C: $3.89 \mathrm{mg}$ of $[\mathrm{Ru}(\mathrm{p}-\mathrm{Cym})(\mathrm{dhbp}) \mathrm{Cl}] \mathrm{Cl}(\mathbf{1})$ in $0.5 \mathrm{~mL}$ of $\mathrm{D}_{2} \mathrm{O}$, after addition of $20 \mu \mathrm{L}$ of $\mathrm{NH}_{3}\left(20 \% \mathrm{v} / \mathrm{v}\right.$ in $\left.\mathrm{H}_{2} \mathrm{O}\right)$. Signals assigned to $[\mathrm{Ru}(\mathrm{p}-\mathrm{Cym})(\mathrm{dhbp}-2 \mathrm{H}) \mathrm{H}]^{-}$have been identified with a green arrow. 


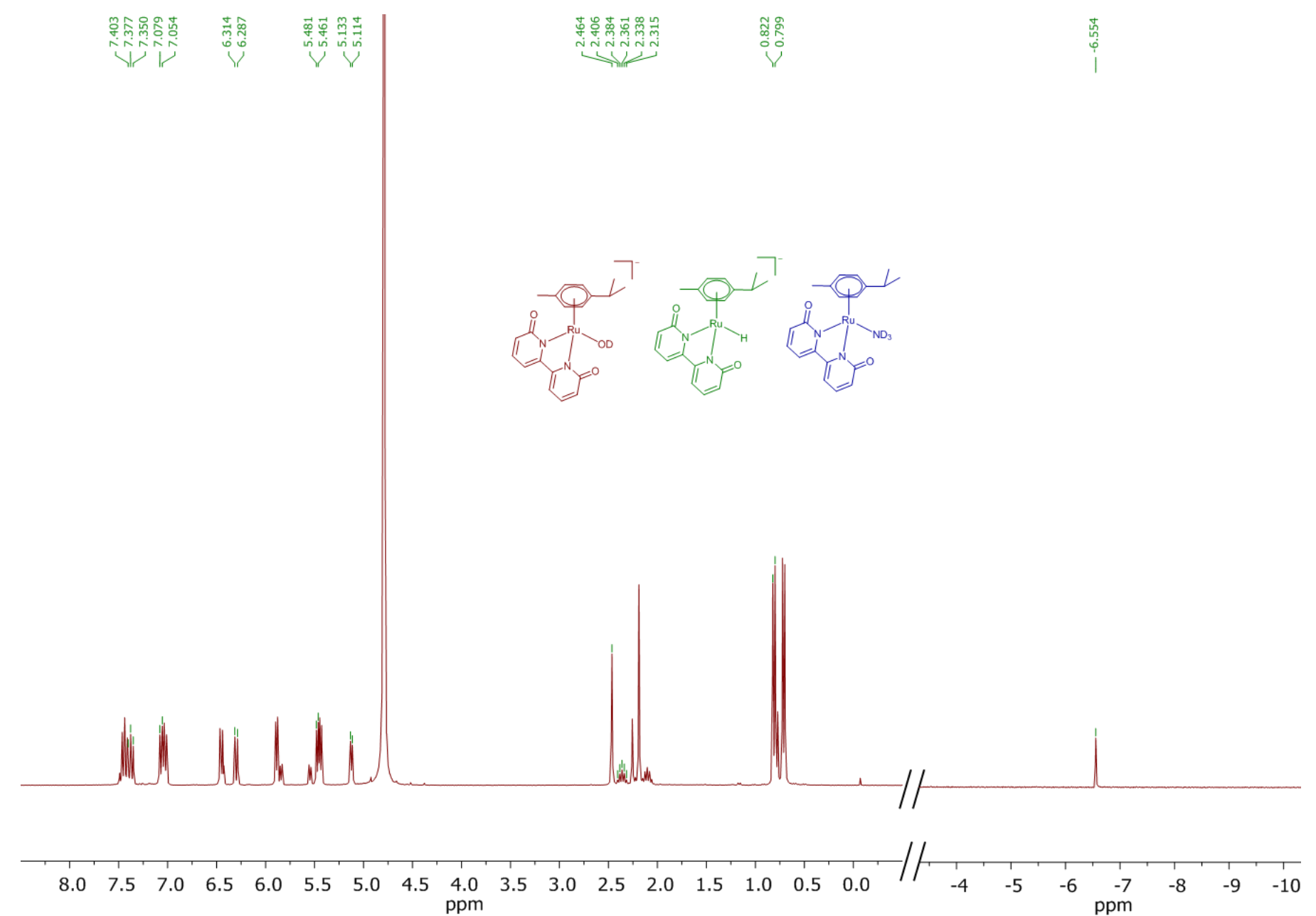

Figure S71. ${ }^{1} \mathrm{H}$ NMR spectra (300 MHz) of $4.03 \mathrm{mg}$ of [Ru(p-Cym)(dhbp)Cl]Cl (1) in $0.5 \mathrm{~mL}$ of $0.001 \mathrm{M} \mathrm{NaOD}$ in $\mathrm{D}_{2} \mathrm{O}, 111 \mathrm{~min}$ after addition of 2 eq. of $\mathrm{AB}$. Only signals assigned to $[\mathrm{Ru}(\mathrm{p}-\mathrm{Cym})(\mathrm{dhbp}-2 \mathrm{H}) \mathrm{H}]^{-}$have been peak-picked. 


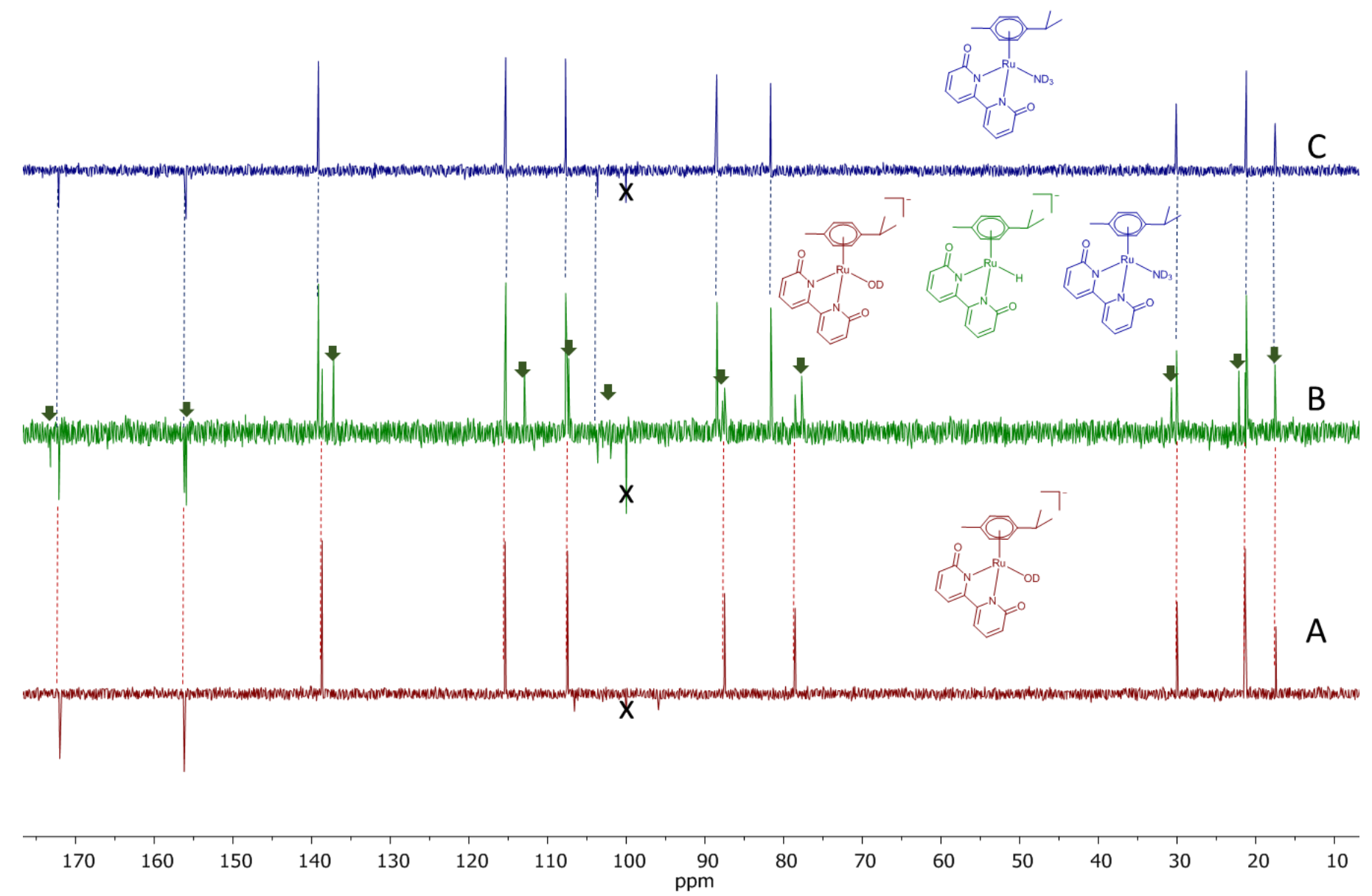

Figure S72. ${ }^{13} \mathrm{C}(\mathrm{APT}) \mathrm{NMR}$ spectra $(75 \mathrm{MHz})$. A: $4.03 \mathrm{mg}$ of $[\mathrm{Ru}(\mathrm{p}-\mathrm{Cym})(\mathrm{dhbp}) \mathrm{Cl}] \mathrm{Cl}(\mathbf{1})$ in $0.5 \mathrm{~mL}$ of $0.001 \mathrm{M} \mathrm{NaOD}$ in $\mathrm{D}_{2} \mathrm{O}$. B: $111 \mathrm{~min}$ after addition of 2 eq. of AB. C: $3.89 \mathrm{mg}$ of $[\mathrm{Ru}(\mathrm{p}-\mathrm{Cym})(\mathrm{dhbp}) \mathrm{Cl}] \mathrm{Cl}(\mathbf{1})$ in $0.5 \mathrm{~mL}$ of $\mathrm{D}_{2} \mathrm{O}$, after addition of $20 \mu \mathrm{L}$ of $\mathrm{NH}_{3}\left(20 \% \mathrm{v} / \mathrm{v}\right.$ in $\left.\mathrm{H}_{2} \mathrm{O}\right)$. Signals assigned to $[\mathrm{Ru}(\mathrm{p}-\mathrm{Cym})(\mathrm{dhbp}-2 \mathrm{H}) \mathrm{H}]^{-}$have been identified with a green arrow. Peak at 100 ppm is an artifact (center of the acquisition window). 


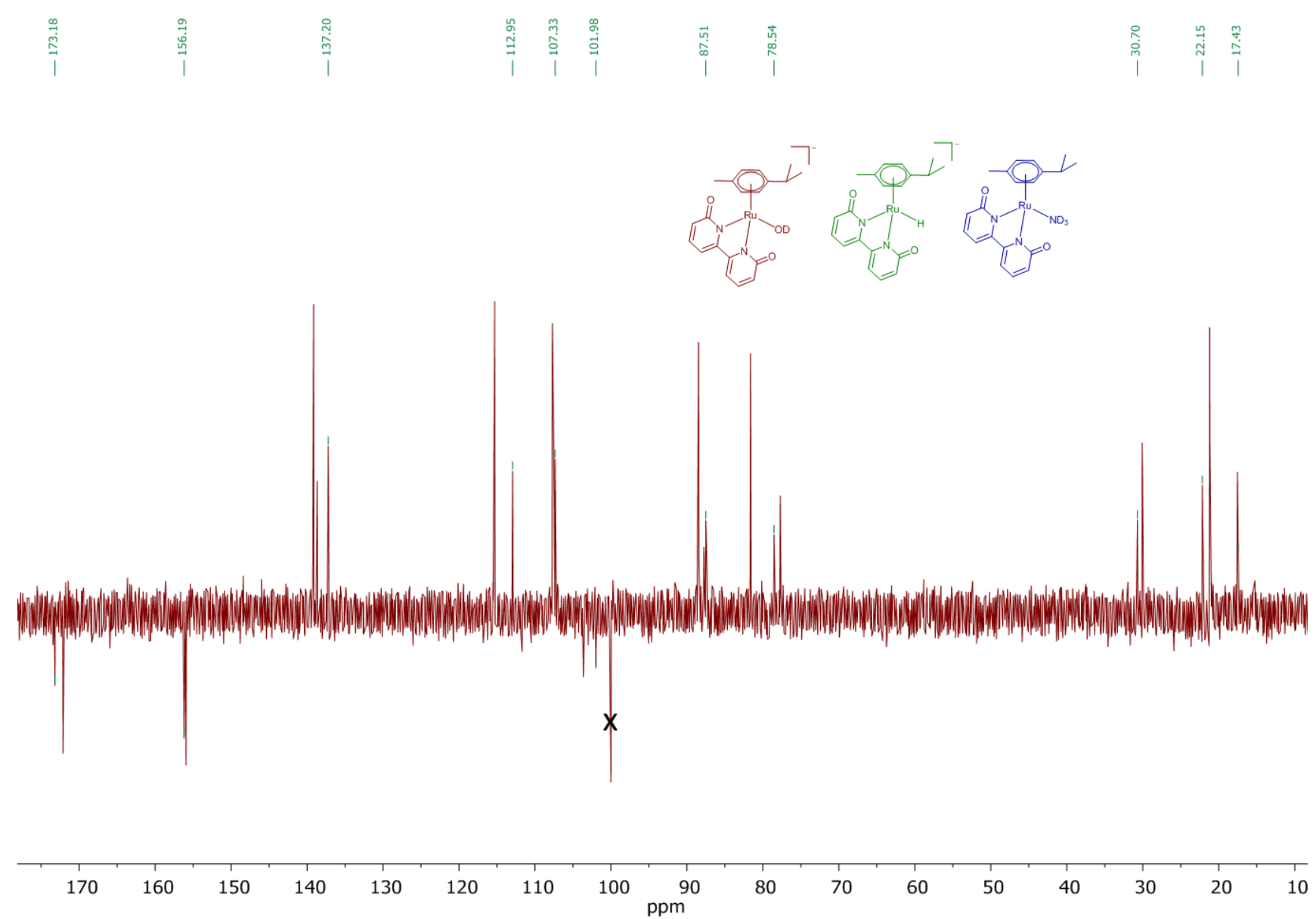

Figure S73. ${ }^{13} \mathrm{C}(\mathrm{APT}) \mathrm{NMR}$ spectra $(75 \mathrm{MHz})$. A: $4.03 \mathrm{mg}$ of $[\mathrm{Ru}(\mathrm{p}-\mathrm{Cym})(\mathrm{dhbp}) \mathrm{Cl}] \mathrm{Cl}(\mathbf{1})$ in $0.5 \mathrm{~mL}$ of $0.001 \mathrm{M} \mathrm{NaOD}$ in $\mathrm{D}_{2} \mathrm{O}$, $111 \mathrm{~min}$ after addition of 2 eq. of $\mathrm{AB}$. Signals assigned to $[\mathrm{Ru}(\mathrm{p}-\mathrm{Cym})(\mathrm{dhbp}-2 \mathrm{H}) \mathrm{H}]^{-}$have been identified with a green arrow. Only signals assigned to $[\mathrm{Ru}(\mathrm{p}-\mathrm{Cym})(\mathrm{dhbp}-2 \mathrm{H}) \mathrm{H}]^{-}$have been peakpicked.One of the quaternary carbons of $\mathrm{p}$-Cymene is hidden in the background noise of the spectra. Peak at $100 \mathrm{ppm}$ is an artifact (center of the acquisition window). 


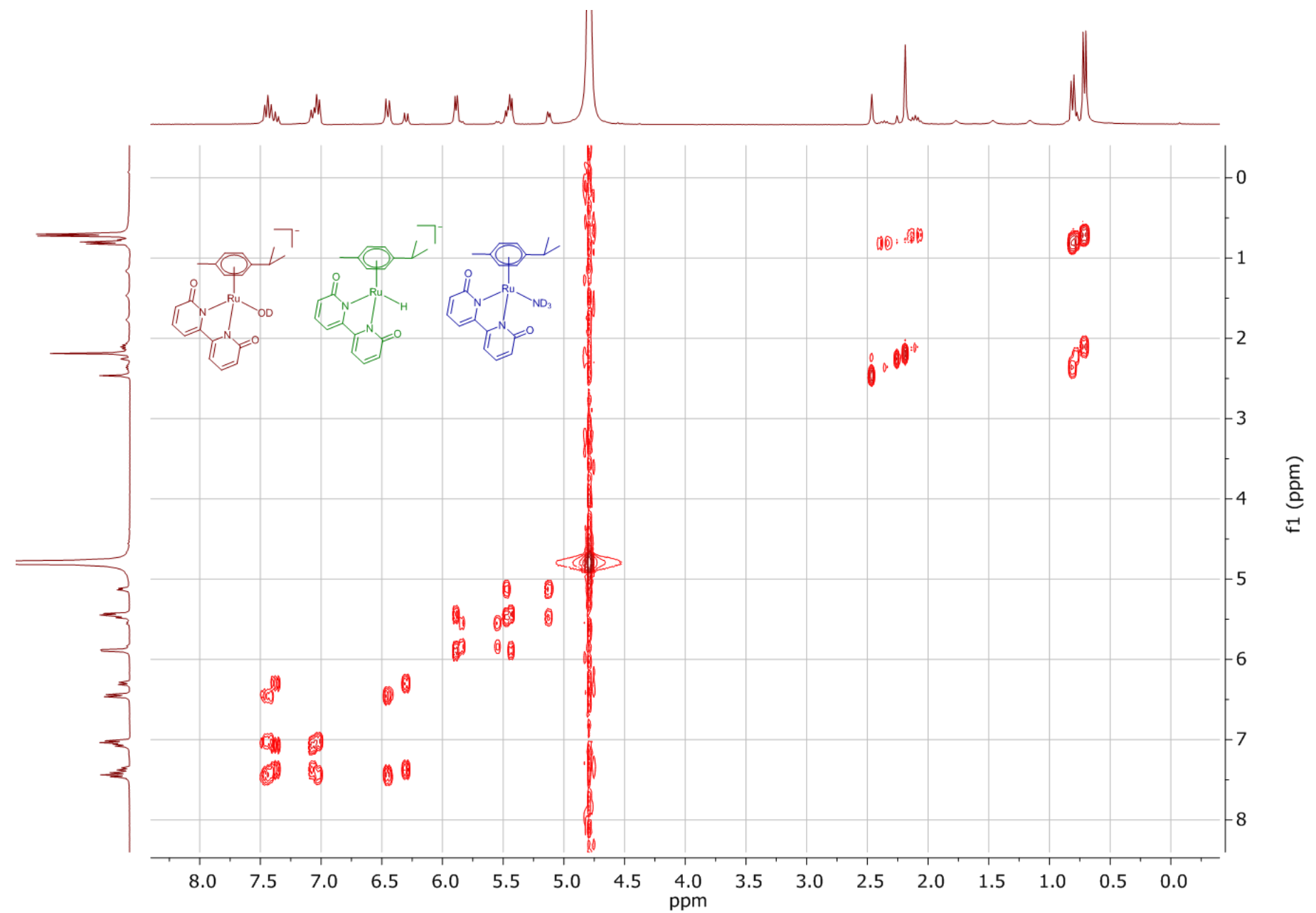

Figure S74. COSY NMR spectra (300 MHz). $4.03 \mathrm{mg}$ of $[R u(p-C y m)(\mathrm{dhbp}) \mathrm{Cl}] \mathrm{Cl}(\mathbf{1})$ in $0.5 \mathrm{~mL}$ of $0.001 \mathrm{M} \mathrm{NaOD}$ in $\mathrm{D}_{2} \mathrm{O}$, after addition of 2 eq. of $\mathrm{AB}$. 
Table S2. ${ }^{1} \mathrm{H}$ NMR chemical shift data $(\mathrm{ppm})$ of $[\mathrm{Ru}(\mathrm{pCym})(\mathrm{dhbp}) \mathrm{L}]^{\mathrm{n}+}$ and $[\mathrm{Ru}(\mathrm{pCym})(\mathrm{dhbp}-2 \mathrm{H}) \mathrm{L}]^{\mathrm{n}-}$ generated in situ.

\begin{tabular}{lccccccccl} 
Comp. & $\mathbf{H}_{\text {Ar1 }}$ & $\mathbf{H}_{\text {Ar2 }}$ & $\mathbf{H}_{\text {Ar3 }}$ & $\mathbf{H}_{\text {pCym1 }}$ & $\mathbf{H}_{\text {pCym2 }}$ & $\mathbf{C H}_{\text {iPr }}$ & $\mathbf{C H}_{\mathbf{3}}$ & iPr & solvent \\
\hline$\left[\mathrm{Ru}(\mathrm{p}-\mathrm{Cym})(\mathrm{dhpb})\left(\mathrm{D}_{2} \mathrm{O}\right)\right]^{2+}$ & 8.01 & 7.79 & 7.20 & 6.30 & 6.04 & 2.31 & 2.24 & 0.88 & $\mathrm{D}_{2} \mathrm{O}$ \\
{$[\mathrm{Ru}(\mathrm{p}-\mathrm{Cym})(\mathrm{dhpb}) \mathrm{Cl}]^{+}$} & 7.98 & 7.83 & 7.19 & 6.23 & 5.83 & 2.38 & 2.27 & 0.89 & $\mathrm{D}_{2} \mathrm{O}$ \\
{$\left[\mathrm{Ru}(\mathrm{p}-\mathrm{Cym})(\mathrm{dhpb}-2 \mathrm{H})\left(\mathrm{D}_{2} \mathrm{O}\right)\right]$} & 7.94 & 7.84 & 7.09 & 6.17 & 5.75 & 2.48 & 2.29 & 0.97 & $\mathrm{CD}_{3} \mathrm{OD}$ \\
{$\left[\mathrm{Ru}(\mathrm{p}-\mathrm{Cym})(\mathrm{dhpb}-2 \mathrm{H})\left(\mathrm{ND}_{3}\right)\right]$} & 7.47 & 7.05 & 6.44 & 5.85 & 5.54 & 2.22 & 2.26 & 0.79 & $\mathrm{D}_{2} \mathrm{O}$ \\
{$[\mathrm{Ru}(\mathrm{p}-\mathrm{Cym})(\mathrm{dhpb}-2 \mathrm{H})(\mathrm{OD})]^{-}$} & 7.37 & 7.01 & 6.45 & 5.93 & 5.56 & 2.27 & 2.32 & 0.87 & $\mathrm{CD} \mathrm{OD}_{3}$ \\
{$[\mathrm{Ru}(\mathrm{p}-\mathrm{Cym})(\mathrm{dhpb}-2 \mathrm{H}) \mathrm{H}]^{-}$} & 7.31 & 7.03 & 6.48 & 5.81 & 5.37 & 2.04 & 2.11 & 0.67 & $\mathrm{NaOD} / \mathrm{D}_{2} \mathrm{O}$ \\
& 7.38 & 7.07 & 6.30 & 5.46 & 5.24 & 2.56 & 2.49 & 1.03 & $\mathrm{CD}_{3} \mathrm{OD}$ \\
& & & & & 5.12 & 2.36 & 2.46 & 0.81 & $\mathrm{NaOD}_{2} \mathrm{O} \mathrm{D}_{2} \mathrm{O}$
\end{tabular}

Spectra description:

Ru- $\mathrm{H}_{2} \mathrm{O}\left(\left[\mathrm{Ru}(\mathrm{p}-\mathrm{Cym})(\mathrm{dhpb})\left(\mathrm{D}_{2} \mathrm{O}\right)\right]^{2+}\right){ }^{1} \mathrm{H}$ NMR $\left(300 \mathrm{MHz}, \mathrm{D}_{2} \mathrm{O}\right) \delta 8.01(\mathrm{t}, \mathrm{J}=8 \mathrm{~Hz}, 2 \mathrm{H}), 7.79$ (dd, J $=7.8,1.0 \mathrm{~Hz}, 2 \mathrm{H}), 7.20(\mathrm{dd}, \mathrm{J}=8.5,0.9 \mathrm{~Hz}, 2 \mathrm{H}), 6.30(\mathrm{~d}, \mathrm{~J}=6.2 \mathrm{~Hz}, 2 \mathrm{H}), 6.04(\mathrm{~d}, \mathrm{~J}=6.0 \mathrm{~Hz}, 2 \mathrm{H})$, 2.31 (hept, J = 7.20 Hz, 1H), $2.24(\mathrm{~s}, 3 \mathrm{H}), 0.88(\mathrm{~d}, \mathrm{~J}=7.0 \mathrm{~Hz}, 6 \mathrm{H}) .{ }^{13} \mathrm{C} \mathrm{NMR}\left(75 \mathrm{MHz}, \mathrm{D}_{2} \mathrm{O}\right) \delta 165.80$, $154.28,142.76,115.23,113.10,105.57,97.05,87.30,82.29,30.13,20.97,17.77$. [119.60 (q, J = $\left.317.2 \mathrm{~Hz}, \mathrm{O}_{3} \mathrm{SCF}_{3}^{-}\right)$]

Ru-Cl ([Ru(p-Cym)(dhpb)Cl] $\left.{ }^{+}\right)^{1} \mathrm{H}$ NMR $\left(300 \mathrm{MHz}, \mathrm{D}_{2} \mathrm{O}\right) \delta 7.98(\mathrm{t}, J=8.0 \mathrm{~Hz}, 2 \mathrm{H}), 7.83(\mathrm{~d}, J=7.7$ $\mathrm{Hz}, 2 \mathrm{H}), 7.19(\mathrm{~d}, J=8.3 \mathrm{~Hz}, 2 \mathrm{H}), 6.23(\mathrm{~d}, J=5.9 \mathrm{~Hz}, 2 \mathrm{H}), 5.83(\mathrm{~d}, J=5.9 \mathrm{~Hz}, 4 \mathrm{H}), 2.38$ (hept, $J=7.0$ $\mathrm{Hz}, 1 \mathrm{H}), 2.27(\mathrm{~s}, 3 \mathrm{H}), 0.89$ (d, $J=6.9 \mathrm{~Hz}, 9 \mathrm{H}) .{ }^{13} \mathrm{C} \mathrm{NMR}(75 \mathrm{MHz}, \mathrm{D} 2 \mathrm{O}) \delta 165.22,153.86,142.19$, $115.70,112.83,87.29,81.93,30.46,21.23$ (quaternary carbons of $p$-Cym not observed).

Ru-Cl ([Ru(p-Cym)(dhpb)Cl $\left.]^{+}\right)^{1} \mathrm{H}$ NMR $\left(300 \mathrm{MHz}, \mathrm{CD}_{3} \mathrm{OD}\right) \delta 7.94(\mathrm{t}, J=8.0 \mathrm{~Hz}, 2 \mathrm{H}), 7.84(\mathrm{dd}, J=$ 7.7, $1.2 \mathrm{~Hz}, 2 \mathrm{H}), 7.09$ (dd, $J=8.1,1.1 \mathrm{~Hz}, 2 \mathrm{H}), 6.17(\mathrm{~d}, J=6.0 \mathrm{~Hz}, 2 \mathrm{H}), 5.75(\mathrm{~d}, J=5.9 \mathrm{~Hz}, 2 \mathrm{H}), 2.48$ (hept, $J=6.9 \mathrm{~Hz}, 1 \mathrm{H}), 2.29(\mathrm{~s}, 3 \mathrm{H}), 0.97(\mathrm{~d}, J=6.9 \mathrm{~Hz}, 6 \mathrm{H}) .{ }^{13} \mathrm{C}$ NMR $\left(75 \mathrm{MHz}, \mathrm{CD}_{3} \mathrm{OD}\right) \delta 167.20$, $155.71,142.76,116.16,113.64,87.99,82.83,32.35,22.44,19.57$ (quaternary carbons of p-Cym not observed).

Ru-NH ${ }_{3}\left(\left[R u(p-C y m)(d h p b-2 H)\left(N_{3}\right)\right]\right){ }^{1} \mathrm{H}$ NMR $\left(300 \mathrm{MHz}, \mathrm{D}_{2} \mathrm{O}\right) \delta 7.47$ (dd, $\left.J=8.5,7.3 \mathrm{~Hz}, 2 \mathrm{H}\right)$, 7.05 (dd, $J=7.4,1.1 \mathrm{~Hz}, 2 \mathrm{H}), 6.44(\mathrm{dd}, J=8.5,1.0 \mathrm{~Hz}, 2 \mathrm{H}), 5.85(\mathrm{~d}, J=5.9 \mathrm{~Hz}, 2 \mathrm{H}), 5.54(\mathrm{~d}, J=6.0$ $\mathrm{Hz}, 2 \mathrm{H}$ ), $2.26(\mathrm{~s}, 3 \mathrm{H}), 2.22$ (hept, $J=7.0 \mathrm{~Hz}, 1 \mathrm{H}), 0.79(\mathrm{~d}, J=6.9 \mathrm{~Hz}, 6 \mathrm{H}) .{ }^{13} \mathrm{C}$ NMR $\left(75 \mathrm{MHz}, \mathrm{D}_{2} \mathrm{O}\right)$ $\delta 218.89,172.14,155.95,139.13,115.32,107.72,103.64,88.50,81.66,30.11,21.20,17.53$.

Ru-NH $\mathbf{N}_{3}\left(\left[\mathrm{Ru}(\mathrm{p}-\mathrm{Cym})(\mathrm{dhpb}-2 \mathrm{H})\left(\mathrm{ND}_{3}\right)\right]\right){ }^{1} \mathrm{H}$ NMR $\left(300 \mathrm{MHz}, \mathrm{CD}_{3} \mathrm{OD}\right) \delta 7.42$ (dd, $\left.J=8.4,7.3 \mathrm{~Hz}, 2 \mathrm{H}\right)$, $7.01(\mathrm{dd}, J=7.3,0.9 \mathrm{~Hz}, 2 \mathrm{H}), 6.45(\mathrm{dd}, J=8.5,0.9 \mathrm{~Hz}, 2 \mathrm{H}), 5.93(\mathrm{~d}, J=6.0 \mathrm{~Hz}, 2 \mathrm{H}), 5.56(\mathrm{~d}, J=6.0$ $\mathrm{Hz}, 2 \mathrm{H}), 2.32(\mathrm{~s}, 3 \mathrm{H}), 2.27$ (hept, $J=7.0 \mathrm{~Hz}, 1 \mathrm{H}), 0.87(\mathrm{~d}, J=6.9 \mathrm{~Hz}, 6 \mathrm{H}) .{ }^{13} \mathrm{C} \mathrm{NMR}\left(75 \mathrm{MHz}, \mathrm{CD}_{3} \mathrm{OD}\right)$ $\delta 173.61,157.42,139.68,116.72,108.16,105.71,101.12,90.25,82.72,31.90,22.48,18.22$.

Ru-OD ([Ru(p-Cym)(dhpb-2H)(OD)] $)^{-1}{ }^{1} \mathrm{H}$ NMR (300 MHz, $300 \mathrm{MHz}, \mathrm{NaOD}$ in $\left.\mathrm{D}_{2} \mathrm{O}, 0.001 \mathrm{M}\right) . \delta$ $7.37(\mathrm{dd}, J=8.4,7.3 \mathrm{~Hz}, 2 \mathrm{H}), 6.96(\mathrm{dd}, J=7.5,1.2 \mathrm{~Hz}, 2 \mathrm{H}), 6.38(\mathrm{dd}, J=8.5,1.1 \mathrm{~Hz}, 2 \mathrm{H}), 5.81(\mathrm{~d}, J$ $=5.8 \mathrm{~Hz}, 2 \mathrm{H}), 5.37(\mathrm{~d}, J=5.7 \mathrm{~Hz}, 2 \mathrm{H}), 2.11(\mathrm{~s}, 3 \mathrm{H}), 2.04$ (hept, $J=6.8 \mathrm{~Hz}, 1 \mathrm{H}), 0.67(\mathrm{~d}, J=6.8 \mathrm{~Hz}$, $6 \mathrm{H}) .{ }^{13} \mathrm{C} \mathrm{NMR}\left(75 \mathrm{MHz}, \mathrm{NaOD}\right.$ in $\left.\mathrm{D}_{2} \mathrm{O}, 0.001 \mathrm{M}\right) \delta 172.01,156.25,138.65,115.41,107.47,106.59$, 95.91, 87.51, 78.54, 30.01, 21.33, 17.43.

Ru-H ([Ru(p-Cym)(dhpb-2H)H] ): ${ }^{1} \mathrm{H}$ NMR (300 MHz, NaOD/D $\left.{ }_{2} \mathrm{O} 0.001 \mathrm{M}\right) \delta 7.38$ (dd, $J=7.6,8.0$ $\mathrm{Hz}, 2 \mathrm{H}), 7.07(\mathrm{~d}, J=7.5 \mathrm{~Hz}, 1 \mathrm{H}), 6.30(\mathrm{~d}, J=7.5 \mathrm{~Hz}, 2 \mathrm{H}), 5.48(\mathrm{~d}, J=6.1 \mathrm{~Hz}, 2 \mathrm{H}), 5.12(\mathrm{~d}, J=6.0 \mathrm{~Hz}$, $2 \mathrm{H}), 2.46(\mathrm{~s}, 3 \mathrm{H}), 2.36$ (hept, $J=6.9 \mathrm{~Hz}, 1 \mathrm{H}), 0.81(\mathrm{~d}, J=7.0 \mathrm{~Hz}, 6 \mathrm{H}),-6.55(\mathrm{~s}, 1 \mathrm{H}) .{ }^{13} \mathrm{C}$ NMR $(75$ $\left.\mathrm{MHz}, \mathrm{NaOD} / \mathrm{D}_{2} \mathrm{O} 0.001 \mathrm{M}\right) \delta 173.18,156.19,137.20,112.95,107.33,101.98,87.51,78.54,30.70$, $22.15,17.43$.

Ru-H ([Ru(p-Cym)(dhpb-2H)H]): ${ }^{1} \mathrm{H}$ NMR $\left(300 \mathrm{MHz}, \mathrm{CD}_{3} \mathrm{OD}\right) \delta 7.31$ (t, $\left.J=7.3 \mathrm{~Hz}, 2 \mathrm{H}\right), 7.03$ (d, J $=7.1 \mathrm{~Hz}, 2 \mathrm{H}), 6.47(\mathrm{~d}, J=8.5 \mathrm{~Hz}, 2 \mathrm{H}), 5.66(\mathrm{~d}, J=5.2 \mathrm{~Hz}, 2 \mathrm{H}), 5.24(\mathrm{~d}, J=5.2 \mathrm{~Hz}, 2 \mathrm{H}), 2.56$ (hept, $J=6.9 \mathrm{~Hz}, 1 \mathrm{H}), 2.49(\mathrm{~s}, 3 \mathrm{H}), 1.03(\mathrm{~d}, J=6.6 \mathrm{~Hz}, 6 \mathrm{H}),-6.0(\mathrm{~s}, 1 \mathrm{H})$. 

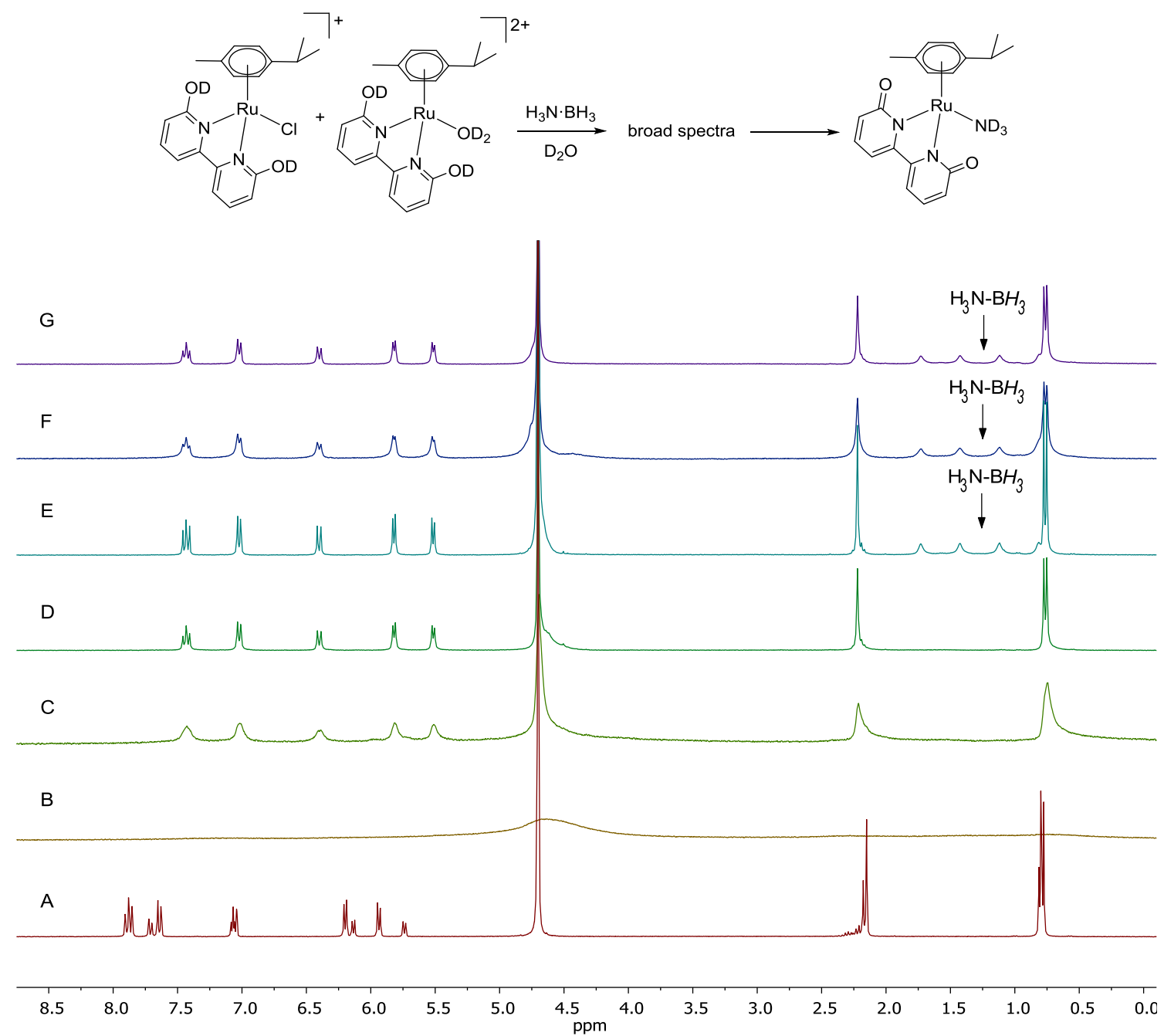

Figure S75. In situ ${ }^{1} \mathrm{H}$ NMR experiment (300 MHz, $\left.\mathrm{D}_{2} \mathrm{O}\right)$. A $0.75 \mathrm{mg}$ of $[\mathrm{Ru}(\mathrm{p}-\mathrm{Cym})(\mathrm{dhbp}) \mathrm{Cl}] \mathrm{Cl}(\mathbf{1})$ in $0.5 \mathrm{~mL}$ of $\mathrm{D}_{2} \mathrm{O}$. B Immediately after addition of 10 eq. of $A B$. $C 13$ minutes after addition of 10 eq. of $A B$. $D 33$ minutes after addition of 10 eq. of $A B$. $E$ Immediately after second addition of 10 eq. of $A B$. $F 19$ minutes after second addition of 10 eq. of $A B$. $F 35$ minutes after second addition of 10 eq. of $A B$. 

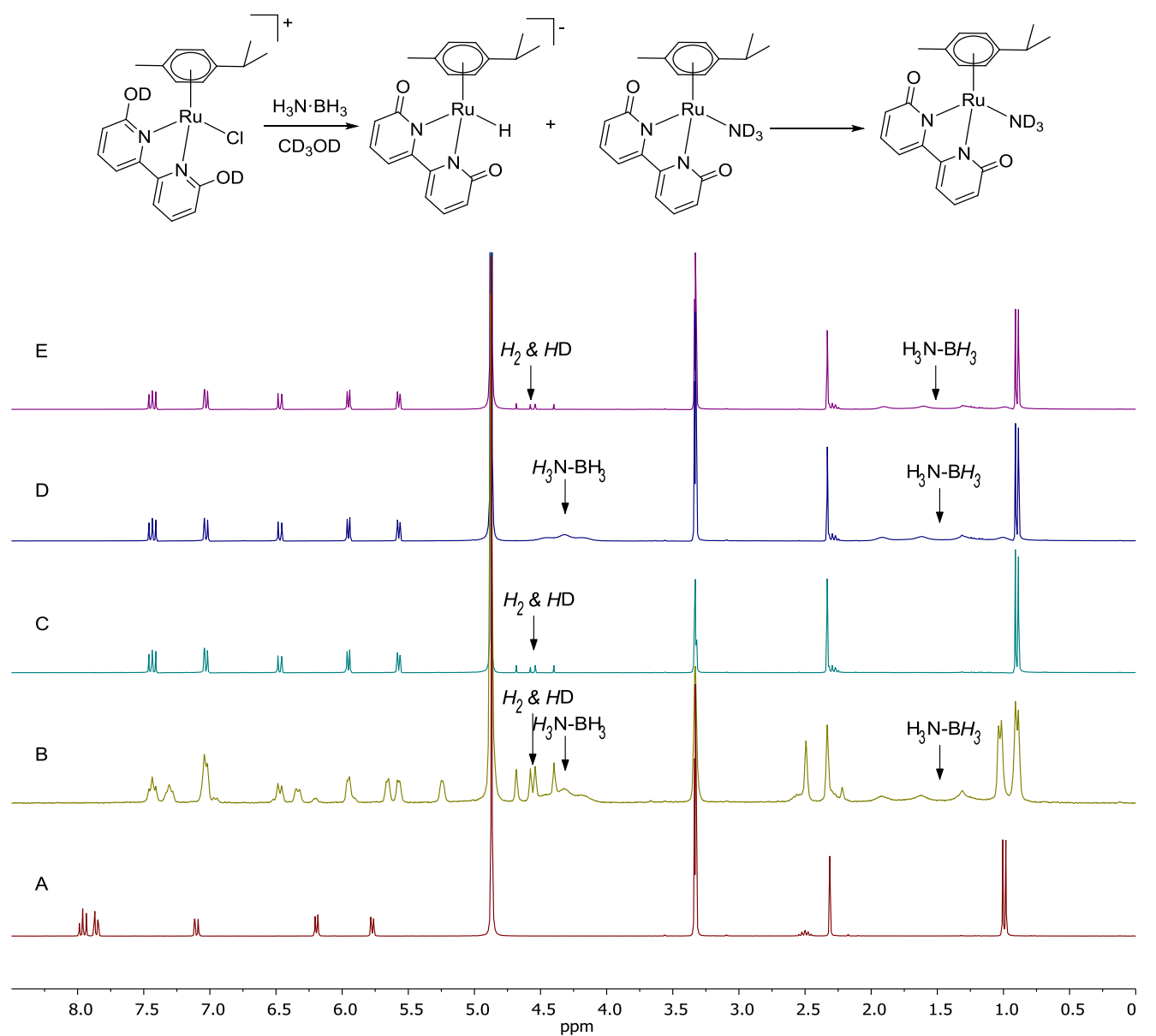

Figure S76. In situ ${ }^{1} \mathrm{H}$ NMR experiment (300 MHz, CD 30 D). A $0.75 \mathrm{mg}$ of [Ru(p-Cym)(dhbp)Cl]Cl (1) in $0.5 \mathrm{~mL}$ of $\mathrm{CD}_{3} \mathrm{OD}$. $\mathrm{B}$ Immediately after addition of 10 eq. of $\mathrm{AB}$. $\mathrm{C} 18$ minutes after addition of 10 eq. of $A B$. $D$ Immediately after second addition of 10 eq. of $A B$. $E 30$ minutes after second addition of 10 eq. of $A B$. Hydride region not shown (see Figure S31). 

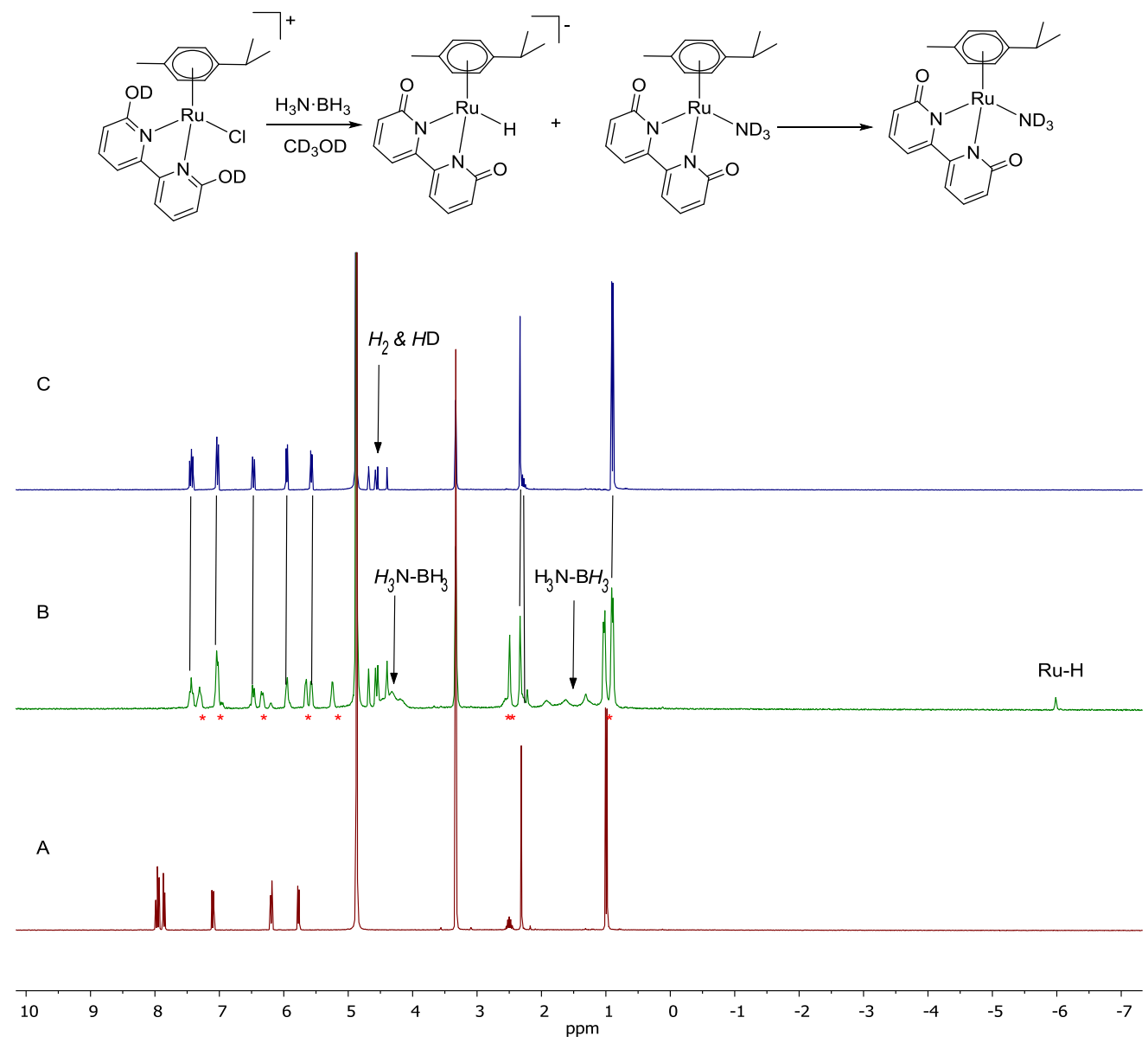

Figure S77. In situ ${ }^{1} \mathrm{H}$ NMR experiment (300 MHz, CD $\left.\mathrm{CD}_{3} \mathrm{OD}\right)$. A $0.75 \mathrm{mg}$ of [Ru(p-Cym)(dhbp)Cl]Cl (1) in $0.5 \mathrm{~mL}$ of $\mathrm{CD}_{3} \mathrm{OD}$. B Immediately after addition of 10 eq. of $\mathrm{AB}$. $\mathrm{C} 3$ minutes after addition of 10 eq. of $A B$. Signals marked with * are attributed to an in situ generated ruthenium-hydride. 

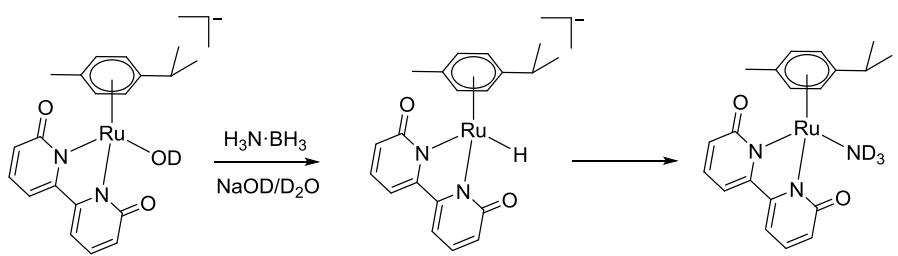

${ }^{1} \mathrm{H}$ NMR
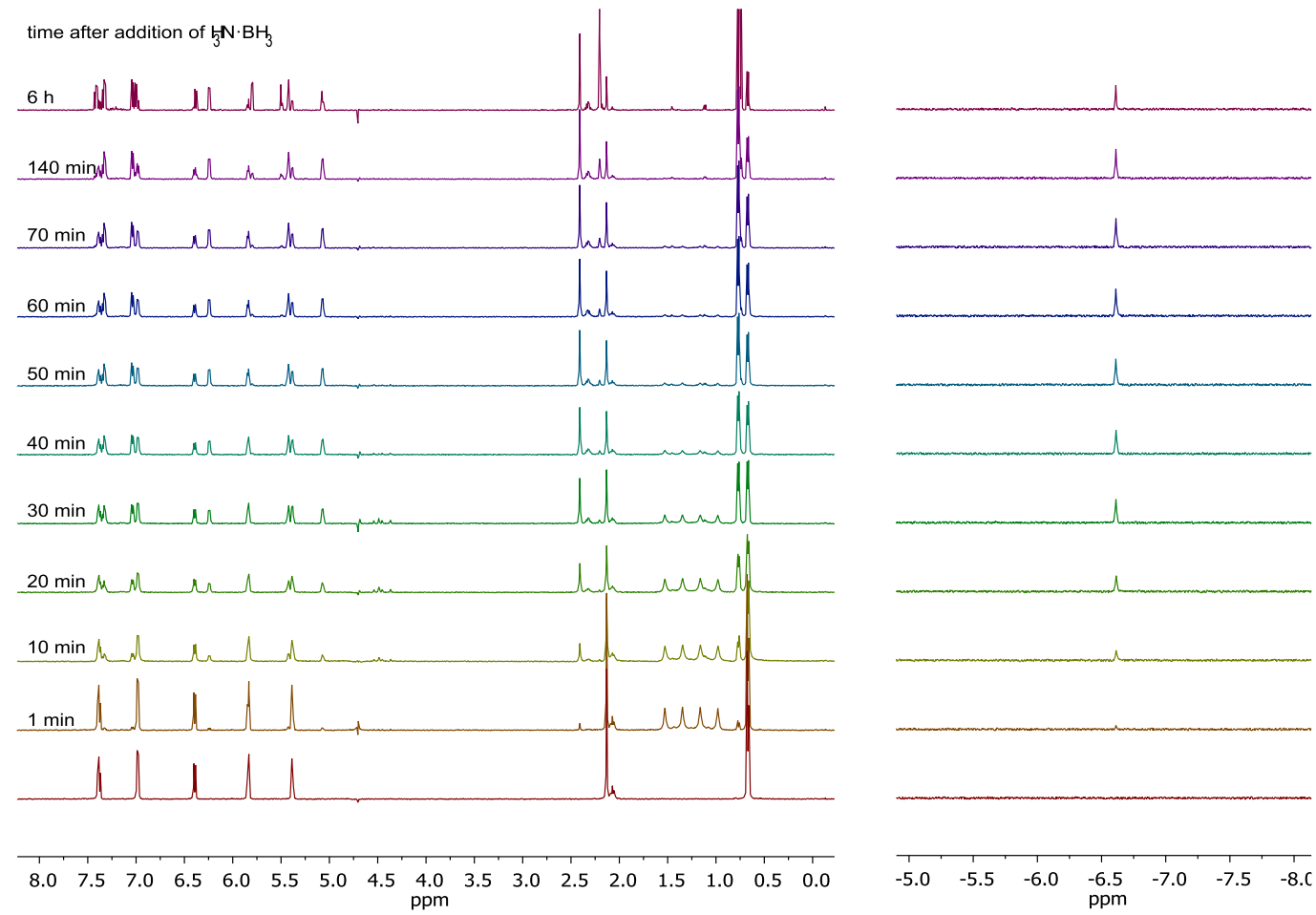

${ }^{11}$ B NMR

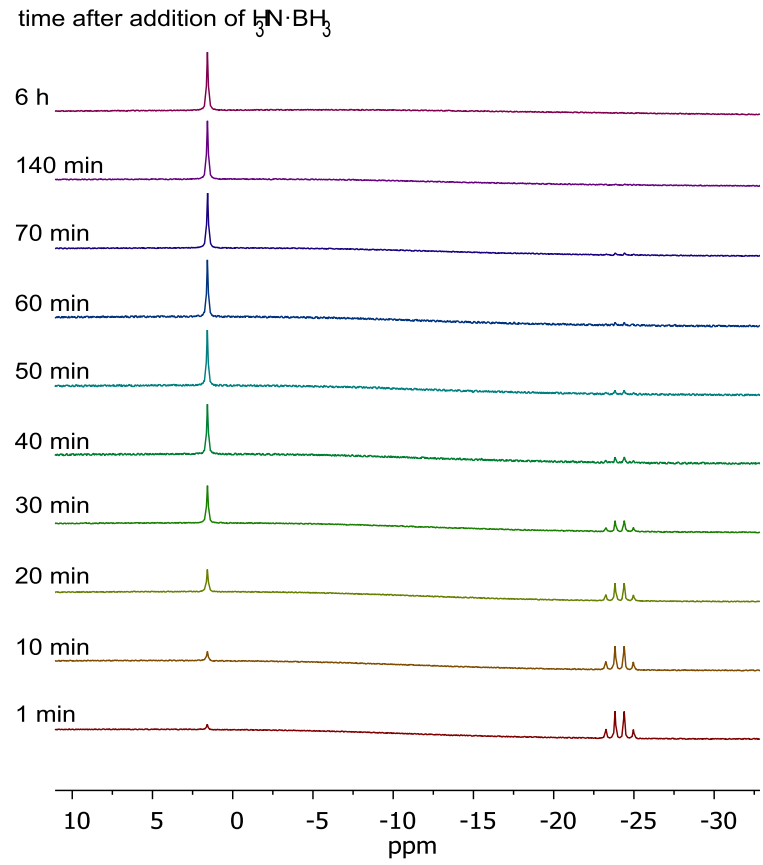

Figure S78. In situ NMR experiment. $0.75 \mathrm{mg}$ of $[\mathrm{Ru}(\mathrm{p}-\mathrm{Cym})(\mathrm{dhbp}) \mathrm{Cl}] \mathrm{Cl}(\mathbf{1})$ in $0.5 \mathrm{~mL}$ of $\mathrm{NaOD} / \mathrm{D}_{2} \mathrm{O} 0.001 \mathrm{M}$. Sequential spectra after addition of 10 eq. of $\mathrm{AB}$. TOP: ${ }^{1} \mathrm{H}$ NMR, water signal suppression (500 MHz) BOTTOM: ${ }^{11} \mathrm{~B}$ NMR $(160 \mathrm{MHz})$ 


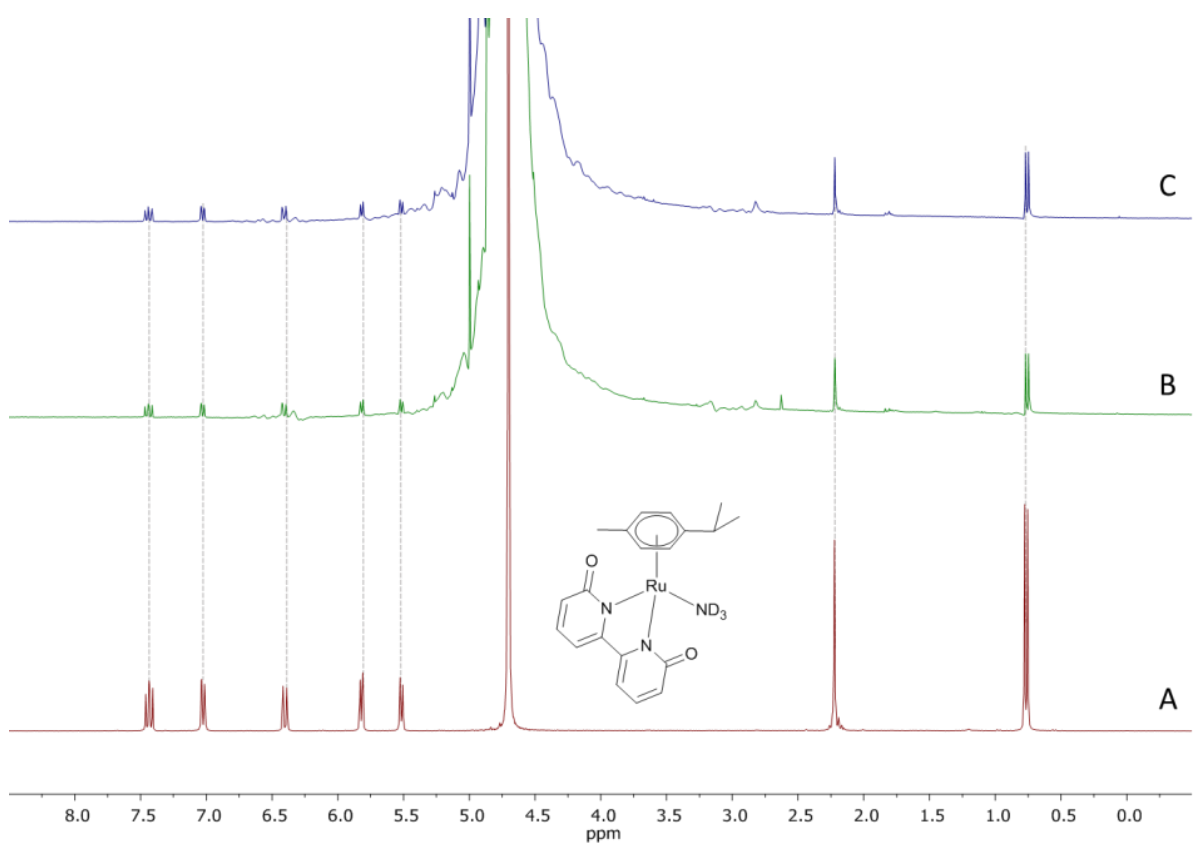

Figure S79. ${ }^{1} \mathrm{H}$ NMR spectra (300 MHz). A. $3.89 \mathrm{mg}$ of $[\mathrm{Ru}(p-C y m)(\mathrm{dhbp}) \mathrm{Cl}] \mathrm{Cl}(\mathbf{1})$ in $0.5 \mathrm{~mL}$ of $\mathrm{D}_{2} \mathrm{O}$ $+20 \mu \mathrm{L} \mathrm{NH} 3$ aq. $20 \% \mathrm{v} / \mathrm{v}$. B. Reaction solution at the end of a catalytic run using [Ru(pCym) (dhbp) $\mathrm{Cl}] \mathrm{Cl}(\mathbf{1})$ as catalyst in $\mathrm{H}_{2} \mathrm{O}$ (internal capillary of $\mathrm{D}_{2} \mathrm{O}$ ). C. Reaction solution at the end of a catalytic run using $\left[\mathrm{Ru}(\mathrm{p}-\mathrm{Cym})(\mathrm{dhbp})\left(\mathrm{H}_{2} \mathrm{O}\right)\right](\mathrm{OTf})_{2}(2)$ as catalyst in $\mathrm{H}_{2} \mathrm{O}$ (internal capillary of $\left.\mathrm{D}_{2} \mathrm{O}\right)$.

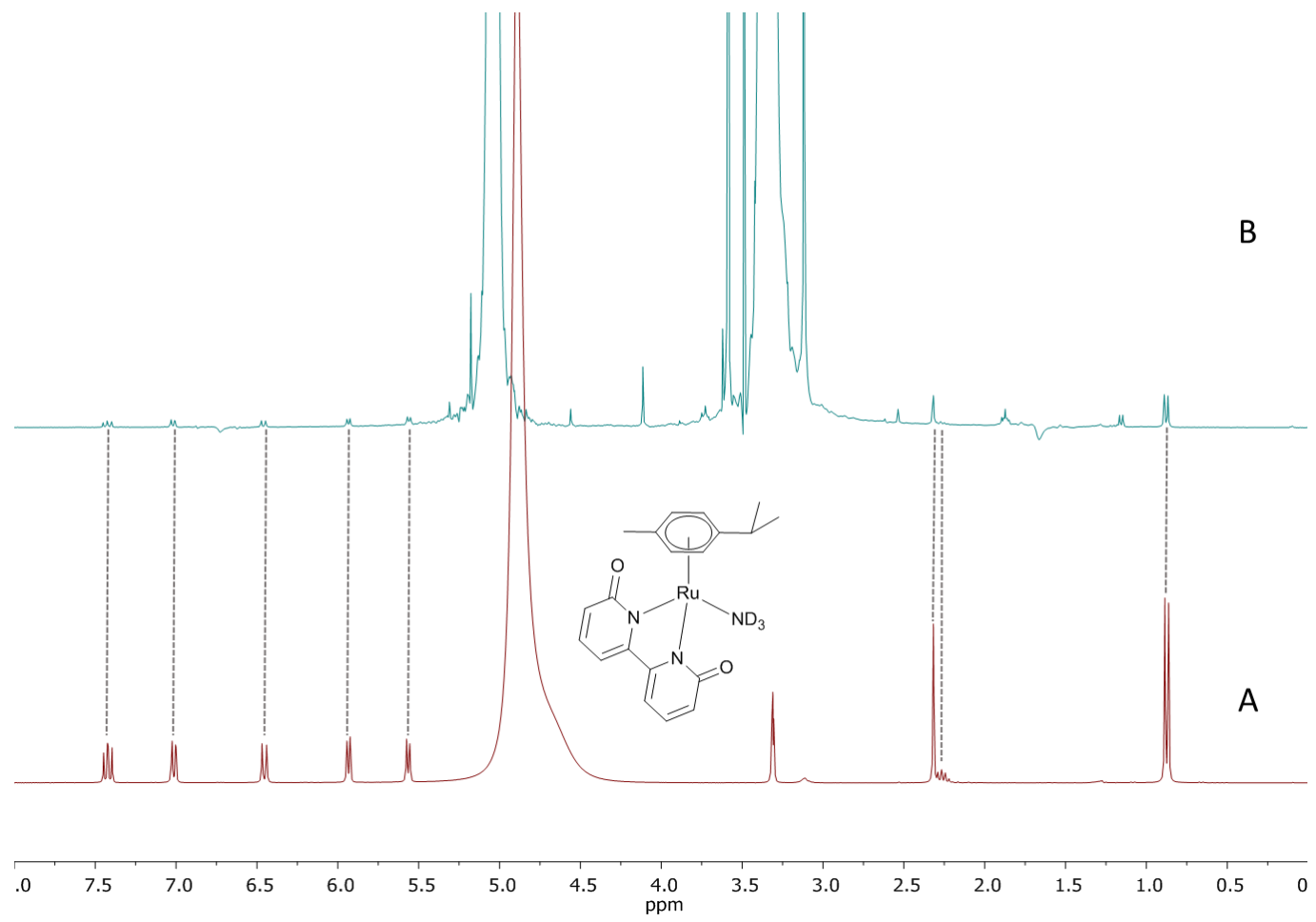

Figure S80. ${ }^{1} \mathrm{H}$ NMR spectra (300 MHz). A $3.85 \mathrm{mg}$ of $[\mathrm{Ru}(\mathrm{p}-\mathrm{Cym})(\mathrm{dhbp}) \mathrm{Cl}] \mathrm{Cl}(\mathbf{1})$ in $0.5 \mathrm{~mL}$ of $\mathrm{CD}_{3} \mathrm{OD}+20 \mu \mathrm{L} \mathrm{NH}$ aq. $20 \%$ v/v. B Reaction solution at the end of a catalytic run using [Ru(pCym) $(\mathrm{dhbp}) \mathrm{Cl}] \mathrm{Cl}(\mathbf{1})$ as catalyst in $\mathrm{CH}_{3} \mathrm{OH}$ (internal capillary of $\mathrm{D}_{2} \mathrm{O}$ ). 


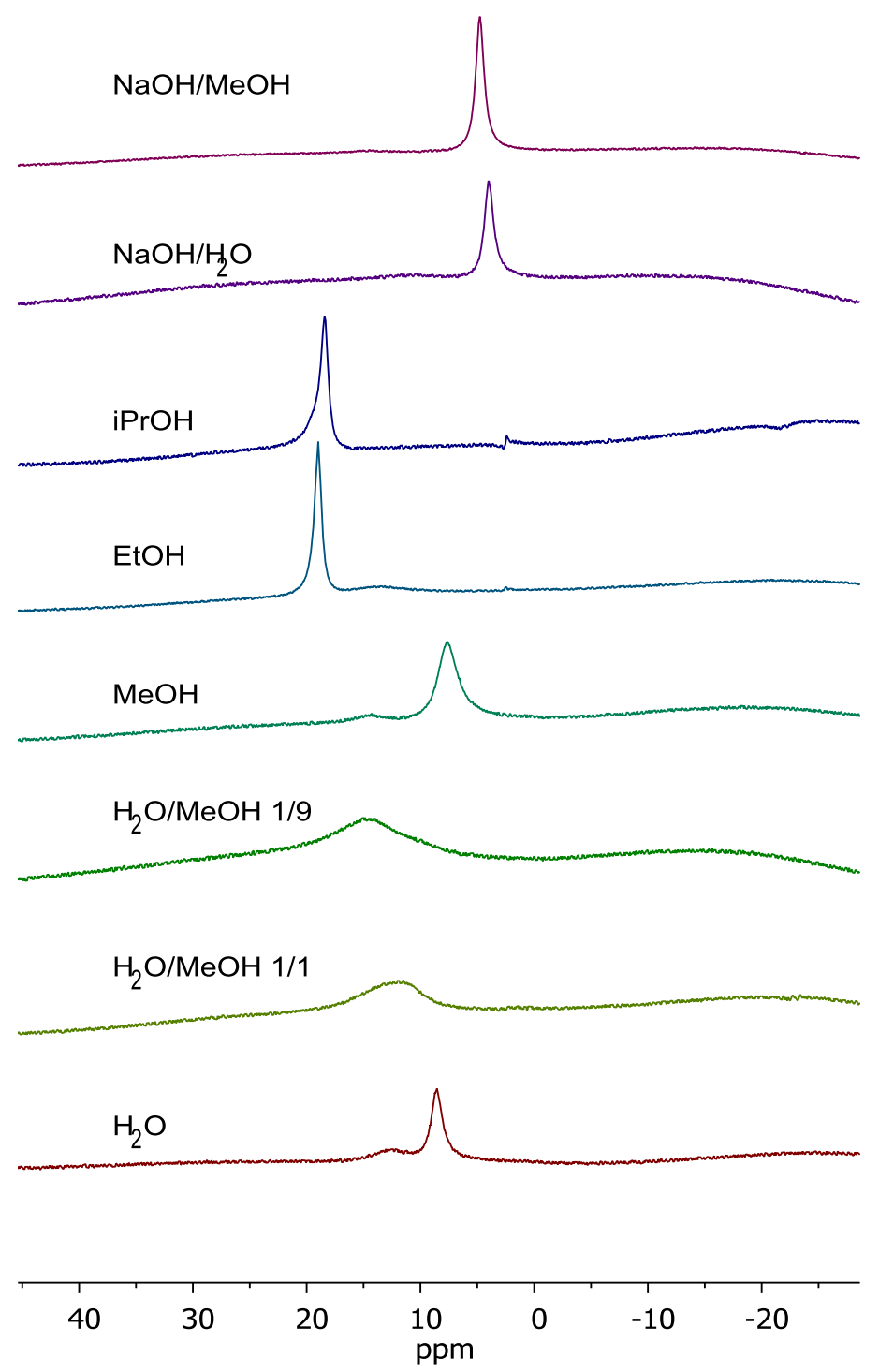

Figure S81. ${ }^{11} \mathrm{~B}$ NMR spectra $(128 \mathrm{MHz})$ of reaction solutions at the end of a catalytic run using $[\mathrm{Ru}(\mathrm{p}-\mathrm{Cym})(\mathrm{dhbp}) \mathrm{Cl}] \mathrm{Cl}(\mathbf{1})$ as catalyst in different solvents. ${ }^{11} \mathrm{~B} N \mathrm{NM}$ spectra at the end of the catalytic reactions show broad bands in the region 2-19 ppm which are characteristic of $\mathrm{H}_{3} \mathrm{BO}_{3}$, $\mathrm{BO}_{2}{ }^{-}$and other borate species in equilibrium in solution, and dependent on the $\mathrm{pH}$ of the reaction media, as described before by other authors. ${ }^{[1-2]}$ 
Mechanistic considerations and DFT calculations.

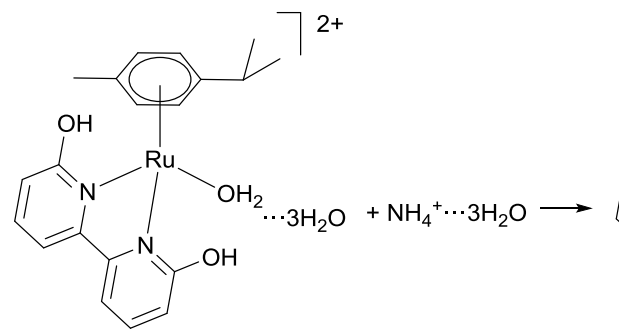

A $\cdots 3 \mathrm{H}_{2} \mathrm{O}$
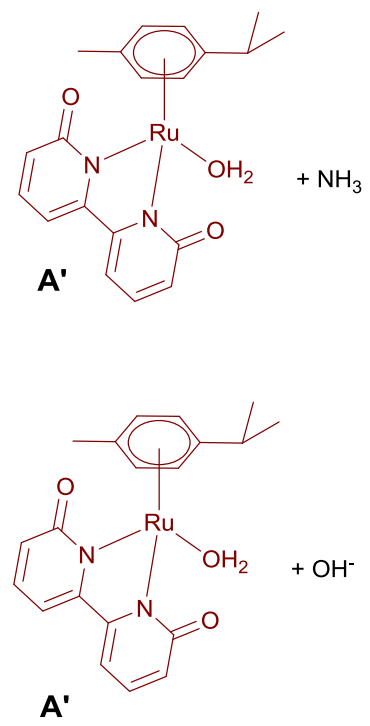

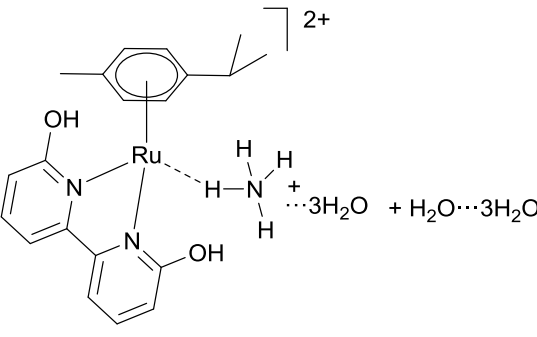

E. - $3 \mathrm{H}_{2} \mathrm{O}$
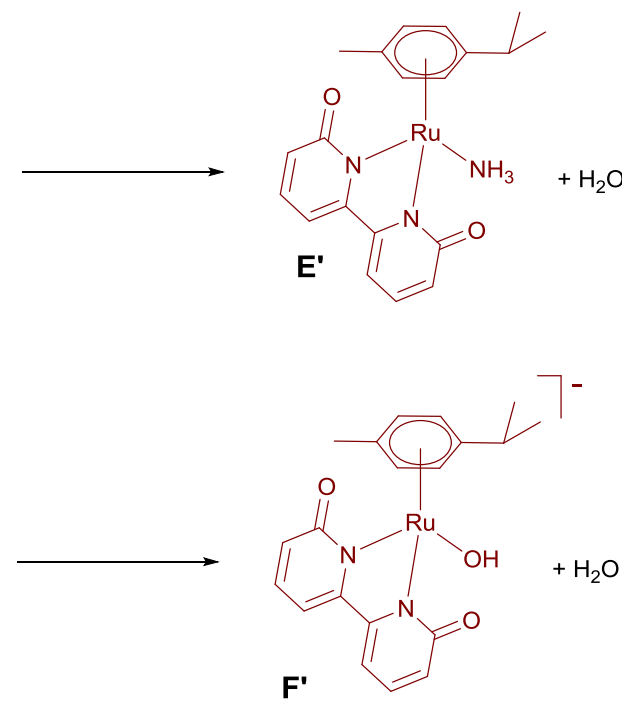

$\Delta \mathrm{G}=20.90 \mathrm{kCal} / \mathrm{mol}$

$\Delta \mathrm{G}=-9.40 \mathrm{kCal} / \mathrm{mol}$

$\Delta \mathrm{G}=-24.78 \mathrm{kCal} / \mathrm{mol}$

Scheme S3. Ligands exchange processes and estimated $\Delta G$, according to DFT calculations. 


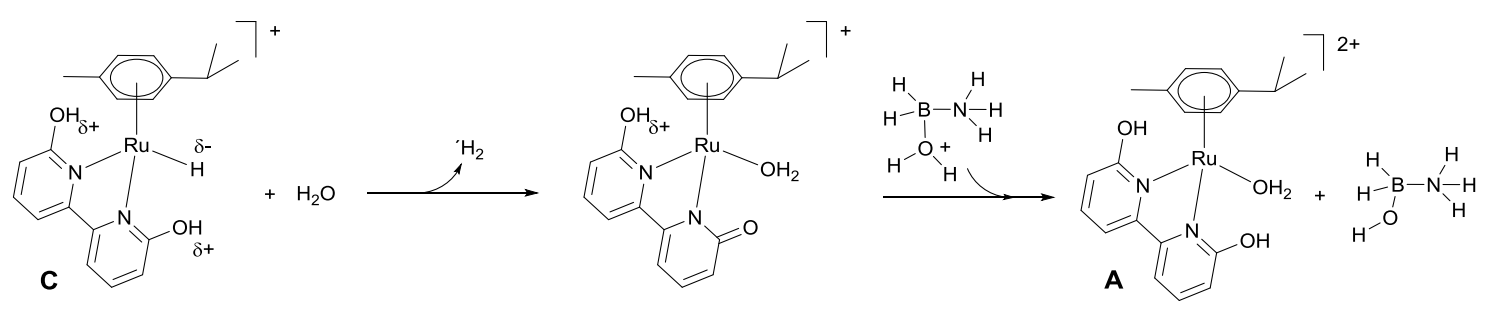

Scheme S4. Alternative reaction pathway for the hydrogen-generation step with dhbpprotonated system.

Table S3. Summary of the energetic values (Hartree) of the calculated species.

\begin{tabular}{|c|c|c|}
\hline species & Hsol & Gsol \\
\hline $\mathrm{OH}^{-}$ & -75.907618 & -75.927174 \\
\hline $\mathrm{NH}_{3}$ & -56.516436 & -56.539314 \\
\hline Ru-OH: $F^{\prime}$ in Figure 6 & -1204.204479 & -1204.282803 \\
\hline $\mathrm{Ru}-\mathrm{NH}_{3}: \mathrm{E}^{\prime}$ in Figure 6 & -1184.791535 & -1184.870430 \\
\hline $\mathrm{Ru}-\mathrm{H}_{2}: \mathbf{D}^{\prime}$ in Figure 6 & -1129.396869 & -1129.473677 \\
\hline Ru-H: $\mathbf{C}^{\prime}$ in Figure 6 & -1128.960399 & -1129.036651 \\
\hline $\mathrm{Ru}-\mathrm{BH}_{3} \mathrm{NH}_{3}: \mathbf{B}^{\prime}$ in Figure 6 & -1211.404513 & -1211.486632 \\
\hline $\mathrm{Ru}-\mathrm{H}_{2} \mathrm{O}: \mathrm{A}^{\prime}$ in Figure 6 & -1204.665720 & -1204.744231 \\
\hline $\mathrm{H}_{2} \mathrm{O} \cdots 3 \mathrm{H}_{2} \mathrm{O}$ & -305.641754 & -305.693080 \\
\hline $\mathrm{NH}_{4}+\cdots 3 \mathrm{H}_{2} \mathrm{O}$ & -286.190072 & -286.242854 \\
\hline $\mathrm{H}_{2}$ & -1.156178 & -1.170957 \\
\hline $\mathrm{HO}-\mathrm{BH}_{2} \mathrm{NH}_{3}$ & -158.406529 & -158.438838 \\
\hline $\mathrm{H}_{2} \mathrm{O}-\mathrm{BH}_{2} \mathrm{NH}_{3}^{+}$ & -158.819703 & -158.853927 \\
\hline $\mathrm{H}_{2} \mathrm{O}$ & -76.406671 & -76.428098 \\
\hline $\mathrm{BH}_{3} \mathrm{NH}_{3}$ & -83.136674 & -83.164781 \\
\hline $\mathrm{Ru}-\mathrm{H}_{2} \mathrm{O} \cdots 3 \mathrm{H}_{2} \mathrm{O}: \mathrm{A} \cdots 3 \mathrm{H}_{2} \mathrm{O}$ in Figure 6 & -1434.780776 & -1434.877283 \\
\hline $\mathrm{Ru}-\mathrm{NH}_{4}+\cdots 3 \mathrm{H}_{2} \mathrm{O}: \mathrm{E} \cdots 3 \mathrm{H}_{2} \mathrm{O}$ in Figure 6 & -1415.295754 & -1415.393755 \\
\hline $\mathrm{Ru}-\mathrm{H}_{2}: \mathrm{D}$ in Figure 6 & -1130.248629 & -1130.326511 \\
\hline $\mathrm{Ru}-\mathrm{H}: \mathrm{C}$ in Figure 6 & -1129.853349 & -1129.930263 \\
\hline $\mathrm{Ru}-\mathrm{BH}_{3} \mathrm{NH}_{3}: \mathrm{B}$ in Figure 6 & -1212.268613 & -1212.352748 \\
\hline $\mathrm{Ru}-\mathrm{H}_{2} \mathrm{O}: \mathrm{A}$ in Figure 6 & -1205.531903 & -1205.612979 \\
\hline
\end{tabular}




\section{Homogeneity tests.}

The homogeneous nature of the catalyst was investigated by running selected catalytic reactions in presence of 1000 eq. of mercury per catalyst. The similar reaction profiles obtained with and without added mercury (within the experimental error) permitted us to confirm the homogeneity of the catalyst during the active catalytic period. The differences observed during the less active period, does not permit us to completely discard that nanoparticles were responsible of part of the activity observed at extended reaction times.
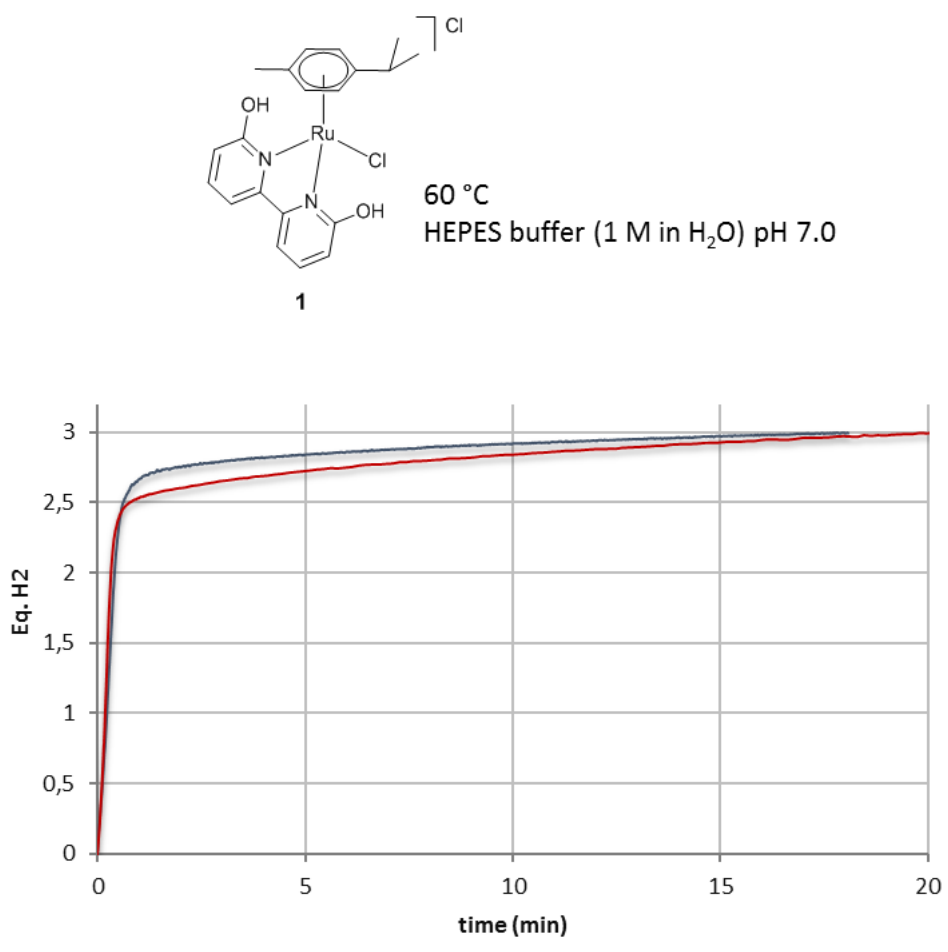

Figure S82. Reaction profiles of $A B$ solvolysis using $[\mathrm{Ru}(\mathrm{p}-\mathrm{Cym})(\mathrm{dhbp}) \mathrm{Cl}] \mathrm{Cl}(\mathbf{1})$. (Table 2, Entry 4). Conditions: blue line: $[\mathrm{Ru}]=2.3 \mathrm{mM},[\mathrm{AB}]=0.46 \mathrm{M}, 1.5 \mathrm{~mL}$ (HEPES buffer $1 \mathrm{M}$ in $\mathrm{H}_{2} \mathrm{O}, \mathrm{pH}$ 7.0), $60{ }^{\circ} \mathrm{C}$. red line: $[\mathrm{Ru}]=2.3 \mathrm{mM},[\mathrm{AB}]=0.46 \mathrm{M}, \mathrm{Hg}$ (1000 equiv. per $\mathrm{Ru}$ ), $1.5 \mathrm{~mL}$ (HEPES buffer $1 \mathrm{M}$ in $\left.\mathrm{H}_{2} \mathrm{O}, \mathrm{pH} 7.0\right), 60^{\circ} \mathrm{C}$. 

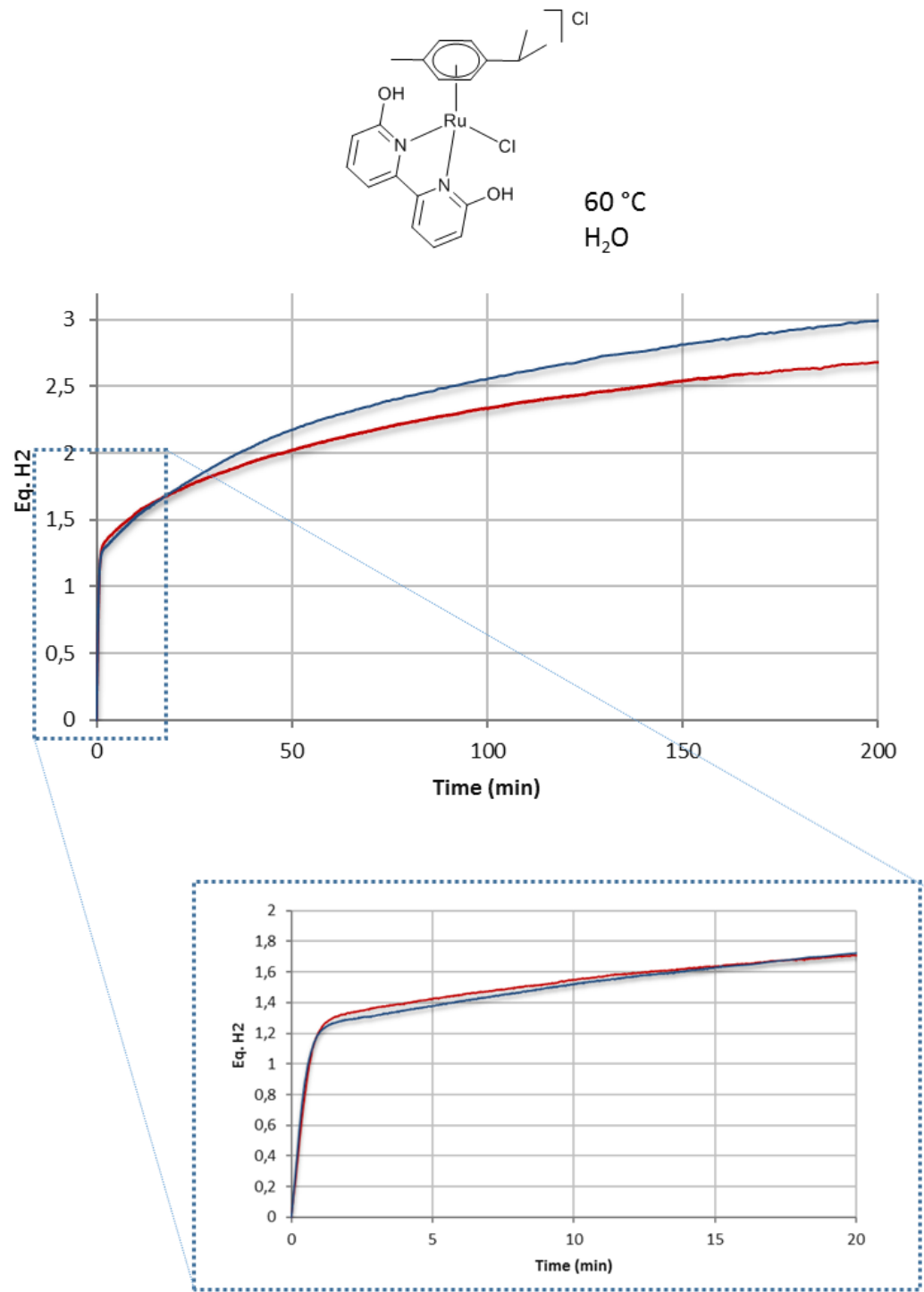

Figure S83. Reaction profiles of $A B$ solvolysis using $[\mathrm{Ru}(\mathrm{p}-\mathrm{Cym})(\mathrm{dhbp}) \mathrm{Cl}] \mathrm{Cl}(\mathbf{1})$. (Table 2, Entry 4). Conditions: blue line: $[\mathrm{Ru}]=2.3 \mathrm{mM},[\mathrm{AB}]=0.46 \mathrm{M}, 1.5 \mathrm{~mL} \mathrm{H} \mathrm{H}_{2} \mathrm{O}, 60^{\circ} \mathrm{C}$. red line: $[\mathrm{Ru}]=2.3$ $\mathrm{mM},[\mathrm{AB}]=0.46 \mathrm{M}, \mathrm{Hg}$ (1000 equiv. per $\mathrm{Ru}), 1.5 \mathrm{~mL} \mathrm{H} \mathrm{O}_{2}, 60^{\circ} \mathrm{C}$.

\section{REFERENCES.}

[1] C. G. Salentine, Inorg. Chem. 1983, 22, 3920-3924.

[2] a) M. Chandra, Q. Xu, J. Power Sources 2006, 156, 190-194; b) M. Chandra, Q. Xu, J. Power Sources 2006, 159, 855-860. 